\title{
Uranyl Nitrate Flow Loop
}

\section{October 2008}

Jennifer L. Ladd-Lively 


\section{DOCUMENT AVAILABILITY}

Reports produced after January 1, 1996, are generally available free via the U.S. Department of Energy (DOE) Information Bridge.

Web site http://www.osti.gov/bridge

Reports produced before January 1, 1996, may be purchased by members of the public from the following source.

National Technical Information Service

5285 Port Royal Road

Springfield, VA 22161

Telephone 703-605-6000 (1-800-553-6847)

TDD 703-487-4639

Fax 703-605-6900

E-mail info@ntis.fedworld.gov

Web site http://www.ntis.gov/support/ordernowabout.htm

Reports are available to DOE employees, DOE contractors, Energy Technology Data Exchange (ETDE) representatives, and International Nuclear Information System (INIS) representatives from the following source.

Office of Scientific and Technical Information

P.O. Box 62

Oak Ridge, TN 37831

Telephone 865-576-8401

Fax 865-576-5728

E-mail reports@osti.gov

Web site http://www.osti.gov/contact.html

This report was prepared as an account of work sponsored by an agency of the United States Government. Neither the United States government nor any agency thereof, nor any of their employees, makes any warranty, express or implied, or assumes any legal liability or responsibility for the accuracy, completeness, or usefulness of any information, apparatus, product, or process disclosed, or represents that its use would not infringe privately owned rights. Reference herein to any specific commercial product, process, or service by trade name, trademark, manufacturer, or otherwise, does not necessarily constitute or imply its endorsement, recommendation, or favoring by the United States Government or any agency thereof. The views and opinions of authors expressed herein do not necessarily state or reflect those of the United States Government or any agency thereof. 
ORNL/TM-2008/048

Nuclear Science and Technology Division

\section{URANYL NITRATE FLOW LOOP}

Jennifer L. Ladd-Lively

Date Published: October 2008

Prepared by OAK RIDGE NATIONAL LABORATORY

P.O. Box 2008

Oak Ridge, Tennessee 37831-6283

managed by

UT-Battelle, LLC

for the

U.S. DEPARTMENT OF ENERGY

under contract DE-AC05-00OR22725 

TABLE OF CONTENTS

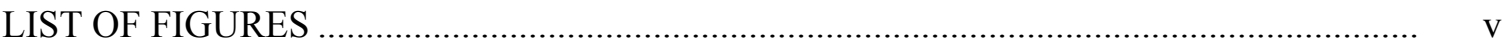

ACRONYMS AND ABBREVIATED TERMINOLOGY …................................................. xi

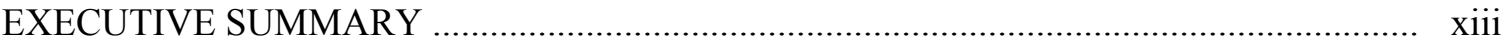

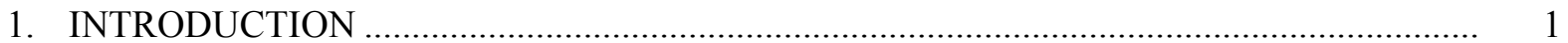

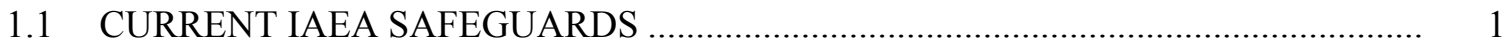

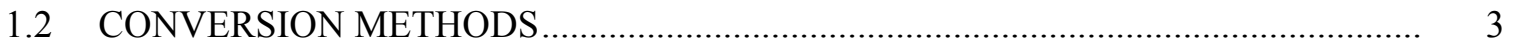

1.3 POTENTIAL PLACES FOR DIVERSION .................................................................... 5

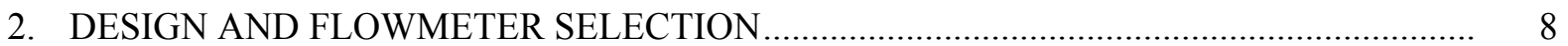

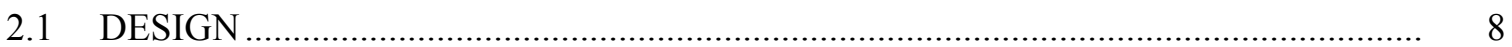

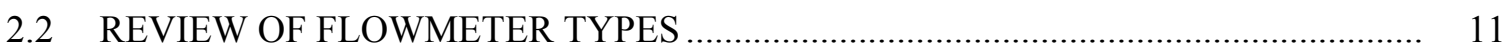

2.2.1 Vortex-Shedding Flowmeters....................................................................... 11

2.2.2 Turbine Flowmeters ............................................................................ 12

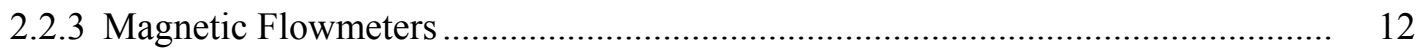

2.2.4 Ultrasonic Flowmeters ...................................................................... 13

2.2.5 Coriolis Flowmeters ............................................................................. 14

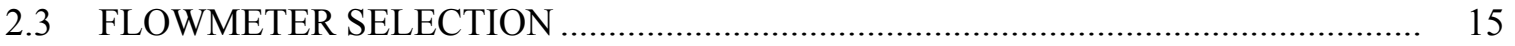

3. OPERATION

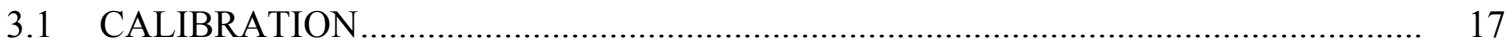

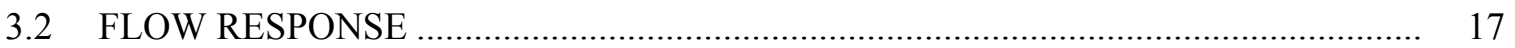

3.3 ENTRAINED AIR RESPONSE ……....................................................... 17

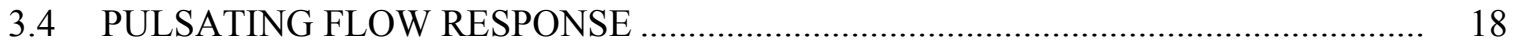

3.5 PULSATING FLOW WITH ENTRAINED AIR RESPONSE .................................. 18

4. RESULTS …

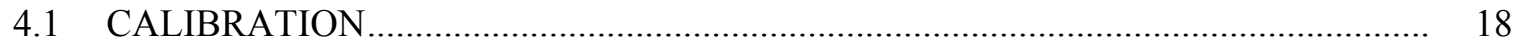

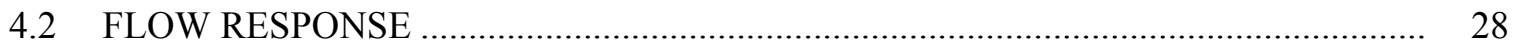

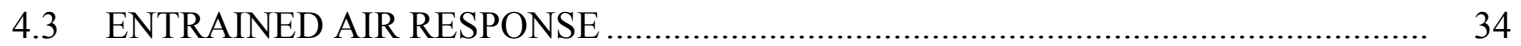

4.4 PULSATING FLOW RESPONSE …................................................................... 34

4.5 PULSATING FLOW WITH ENTRAINED AIR RESPONSE ................................... 35

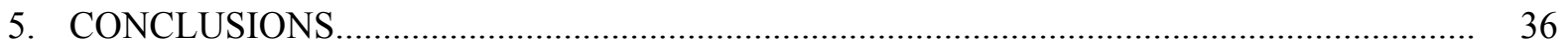

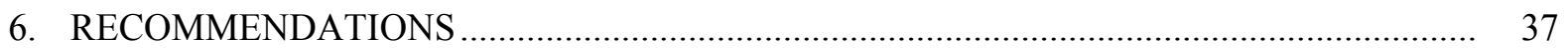

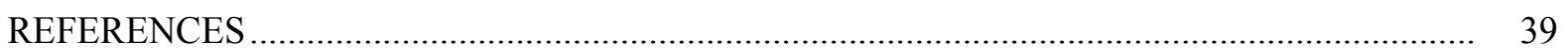

APPENDIX A: FLOWMETERS CONSIDERED FOR TESTING ............................................. A-1

APPENDIX B: GRAPHS OF 8-h STEADY-STATE FLOW ….................................................. B-1 
APPENDIX C: GRAPHS OF ENTRAINED AIR RESPONSE ............................................. C-1

APPENDIX D: GRAPHS OF PULSATING FLOW RESPONSE ............................................ D-1

APPENDIX E: GRAPHS OF PULSATING FLOW WITH ENTRAINED AIR …...................... E-1 


\section{LIST OF FIGURES}

Figure

Page

1 Simplified flow diagram of the wet solvent extraction process used to convert yellowcake to $\mathrm{UF}_{6}$ or uranium metal.......................................................... 4

2 Potential diversion paths for intermediate products from an NUCP .......................... 6

3 Uranium conversion process for a small-size plant with proposed safeguards monitoring points identified ....................................................................... $\quad 7$

$4 \quad$ Simplified diagram of the uranyl nitrate flow loop ........................................... 9

$5 \quad$ Complete uranyl nitrate flow loop system....................................................... 10

$6 \quad$ Close-up view of instruments installed in the uranyl nitrate flow loop...................... 10

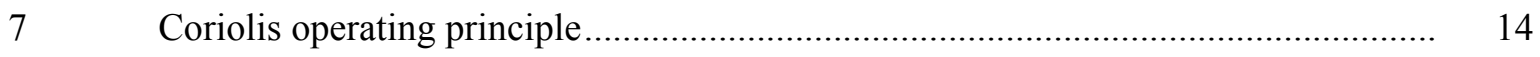

$8 \quad$ Flowmeters selected for installation in the ORNL uranyl nitrate flow loop .............. 16

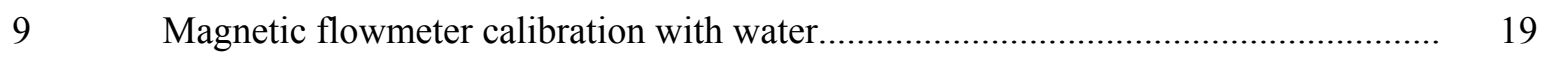

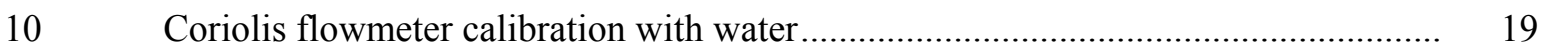

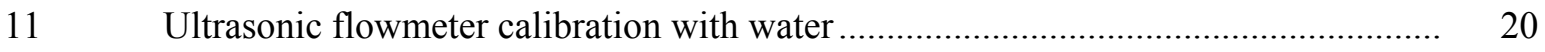

12 Initial magnetic flowmeter calibration with uranyl nitrate ...................................... 21

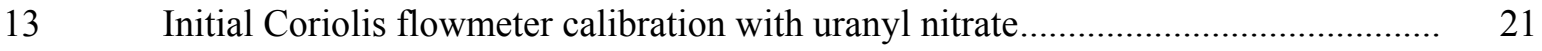

14 Magnetic flowmeter calibration with uranyl nitrate following pump failure in August 2005 ............................................................................ 22

15 Coriolis flowmeter calibration with uranyl nitrate following pump failure in August 2005

16 Magnetic flowmeter calibration with uranyl nitrate following installation of new pump head.

17 Coriolis flowmeter calibration with uranyl nitrate following installation of new pump head.

18 Magnetic flowmeter calibration with uranyl nitrate following air entrainment testing 
19 Coriolis flowmeter calibration with uranyl nitrate following

air entrainment testing

20 Magnetic flowmeter calibration with uranyl nitrate and

organic contamination

21 Coriolis flowmeter calibration with uranyl nitrate and

organic contamination

22 Final magnetic flowmeter calibration.

23 Final Coriolis flowmeter calibration

24 Magnetic flowmeter response over flow range

Coriolis flowmeter response over flow range.

Magnetic flowmeter response at low pump speeds

30

Coriolis flowmeter response at low pump speeds

Magnetic flowmeter response at low pump speeds $(3.6-7.2 \mathrm{~Hz}$ ) for $15 \mathrm{~min}$

31 Coriolis flowmeter response at low pump speeds $(8.3-11.9 \mathrm{~Hz})$ for $15 \mathrm{~min}$

32 Magnetic flowmeter response to entrained air $(500 \mathrm{sccm})$ at a pump speed of $8.3 \mathrm{~Hz}$

$34 \quad$ Magnetic flowmeter response to pulsating flow with entrained air at a pump speed of $9.5 \mathrm{~Hz}$.

35 Magnetic meter response to pump speeds of 2-12 Hz

36 Coriolis meter response to pump speeds of $2-12 \mathrm{~Hz}$

B.1 Magnetic response to steady-state flow at $3.6 \mathrm{~Hz}$ for 8 -h continuous operation...

B.2 Coriolis response to steady-state flow at $3.6 \mathrm{~Hz}$ for 8 -h continuous operation

B.3 Magnetic response to steady-state flow at $4.8 \mathrm{~Hz}$ for 8 -h continuous operation..........

B.4 Coriolis response to steady-state flow at $4.8 \mathrm{~Hz}$ for 8 -h continuous operation............ 
B.6 Coriolis response to steady-state flow at $6.0 \mathrm{~Hz}$ for 8 -h continuous operation........... B-5

B.7 Magnetic response to steady-state flow at $7.2 \mathrm{~Hz}$ for 8 -h continuous operation......... B-6

B.8 Coriolis response to steady-state flow at $7.2 \mathrm{~Hz}$ for 8 -h continuous operation........... B-6

B.9 Magnetic response to steady-state flow at $8.3 \mathrm{~Hz}$ for 8 -h continuous operation......... B-7

B.10 Coriolis response to steady-state flow at $8.3 \mathrm{~Hz}$ for 8 -h continuous operation........... B-7

B.11 Magnetic response to steady-state flow at $9.5 \mathrm{~Hz}$ for 8 -h continuous operation......... B-8

B.12 Coriolis response to steady-state flow at $9.5 \mathrm{~Hz}$ for 8 -h continuous operation........... B-8

B.13 Magnetic response to steady-state flow at $10.7 \mathrm{~Hz}$ for 8 -h continuous operation....... B-9

B.14 Coriolis response to steady-state flow at $10.7 \mathrm{~Hz}$ for 8 -h continuous operation......... B-9

B.15 Magnetic response to steady-state flow at $11.9 \mathrm{~Hz}$ for 8 -h continuous operation....... B-10

B.16 Coriolis response to steady-state flow at $11.9 \mathrm{~Hz}$ for 8 -h continuous operation......... B-10

C.1 Magnetic response to entrained air $(500 \mathrm{sccm})$ and pump speed of $3.6 \mathrm{~Hz} \ldots \ldots \ldots \ldots \ldots . . . . . \quad \mathrm{C}-3$

C.2 Coriolis response to entrained air $(500 \mathrm{sccm})$ and pump speed of $3.6 \mathrm{~Hz} \ldots \ldots \ldots \ldots \ldots \ldots . . . . . . .3$

C.3 Magnetic response to entrained air (500 sccm) and pump speed of $4.8 \mathrm{~Hz} \ldots \ldots \ldots \ldots \ldots . . . . . \quad \mathrm{C}-4$

C.4 Coriolis response to entrained air $(500 \mathrm{sccm})$ and pump speed of $4.8 \mathrm{~Hz} \ldots \ldots \ldots \ldots \ldots . . . . . . . .4$

C.5 Magnetic response to entrained air $(500 \mathrm{sccm})$ and pump speed of $6.0 \mathrm{~Hz} \ldots \ldots \ldots \ldots \ldots . . . . . \quad \mathrm{C}-5$

C.6 Coriolis response to entrained air $(500 \mathrm{sccm})$ and pump speed of $6.0 \mathrm{~Hz} \ldots \ldots \ldots \ldots \ldots . . . . . . . .5$

C.7 Magnetic response to entrained air $(500 \mathrm{sccm})$ and pump speed of $7.2 \mathrm{~Hz} \ldots \ldots \ldots \ldots \ldots . . . . \quad \mathrm{C}-6$

C.8 Coriolis response to entrained air $(500 \mathrm{sccm})$ and pump speed of $7.2 \mathrm{~Hz} \ldots \ldots \ldots \ldots \ldots \ldots$

C.9 Magnetic response to entrained air $(500 \mathrm{sccm})$ and pump speed of $8.3 \mathrm{~Hz} \ldots \ldots \ldots \ldots \ldots . . . . \quad \mathrm{C}-7$

C.10 Coriolis response to entrained air $(500 \mathrm{sccm})$ and pump speed of $8.3 \mathrm{~Hz} \ldots \ldots \ldots \ldots \ldots . . . . . . . .7$

C.11 Magnetic response to entrained air $(500 \mathrm{sccm})$ and pump speed of $9.5 \mathrm{~Hz} \ldots \ldots \ldots \ldots \ldots . . . . . \quad \mathrm{C}-8$

C.12 Coriolis response to entrained air $(500 \mathrm{sccm})$ and pump speed of $9.5 \mathrm{~Hz} \ldots \ldots \ldots \ldots \ldots . . . . . . \quad \mathrm{C}-8$

C.13 Magnetic response to entrained air $(500 \mathrm{sccm})$ and pump speed of $10.7 \mathrm{~Hz} \ldots \ldots \ldots \ldots . . . . \quad \mathrm{C}-9$

C.14 Coriolis response to entrained air $(500 \mathrm{sccm})$ and pump speed of $10.7 \mathrm{~Hz} \ldots \ldots \ldots \ldots \ldots . . . . . \quad \mathrm{C}-9$ 
C.15 Magnetic response to entrained air $(500 \mathrm{sccm})$ and pump speed of $11.9 \mathrm{~Hz} \ldots \ldots \ldots \ldots . . . . \mathrm{C}-10$

C.16 Coriolis response to entrained air $(500 \mathrm{sccm})$ and pump speed of $11.9 \mathrm{~Hz} \ldots \ldots \ldots \ldots \ldots . . . . . .10$

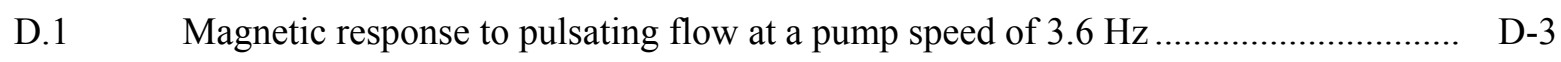

D.2 Coriolis response to pulsating flow at a pump speed of $3.6 \mathrm{~Hz}$............................. D-3

D.3 Magnetic response to pulsating flow at a pump speed of $4.8 \mathrm{~Hz}$............................ D-4

D.4 Coriolis response to pulsating flow at a pump speed of $4.8 \mathrm{~Hz}$............................ D-4

D.5 Magnetic response to pulsating flow at a pump speed of $6.0 \mathrm{~Hz}$............................ D-5

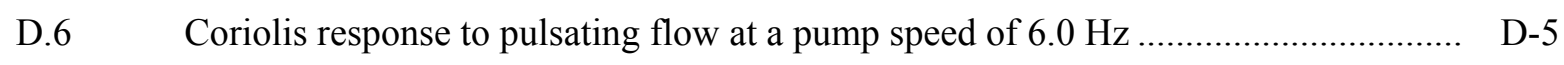

D.7 Magnetic response to pulsating flow at a pump speed of $7.2 \mathrm{~Hz}$............................ D-6

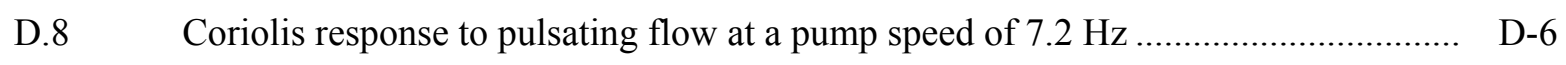

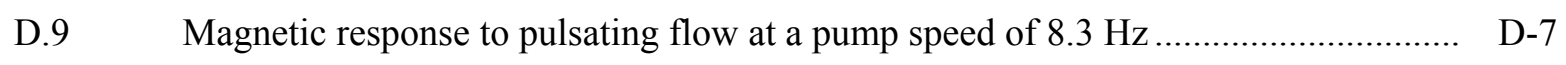

D.10 Coriolis response to pulsating flow at a pump speed of $8.3 \mathrm{~Hz}$........................... D-7

D.11 Magnetic response to pulsating flow at a pump speed of $9.5 \mathrm{~Hz}$........................... D-8

D.12 Coriolis response to pulsating flow at a pump speed of $9.5 \mathrm{~Hz}$........................... D-8

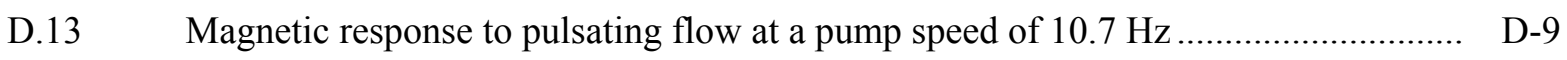

D.14 Coriolis response to pulsating flow at a pump speed of $10.7 \mathrm{~Hz}$.......................... D-9

D.15 Magnetic response to pulsating flow at a pump speed of $11.9 \mathrm{~Hz}$........................... D-10

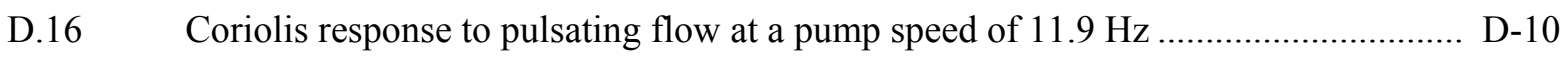

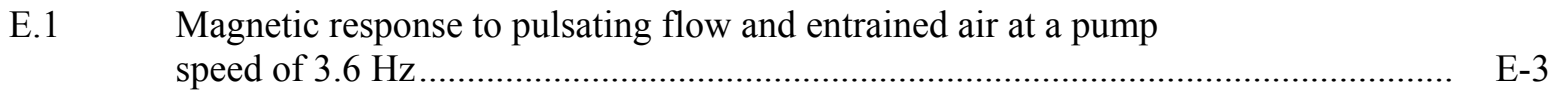

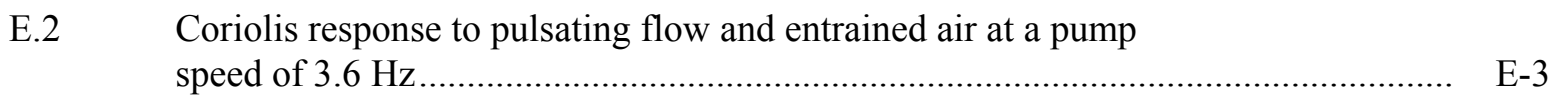

E.3 Magnetic response to pulsating flow and entrained air at a pump

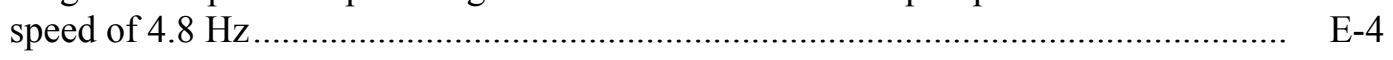

E.4 Coriolis response to pulsating flow and entrained air at a pump speed of $4.8 \mathrm{~Hz}$. 
E.5 Magnetic response to pulsating flow and entrained air at a pump speed of $6.0 \mathrm{~Hz}$.

E.6 Coriolis response to pulsating flow and entrained air at a pump speed of $6.0 \mathrm{~Hz}$.

E.7 Magnetic response to pulsating flow and entrained air at a pump speed of $7.2 \mathrm{~Hz}$.

E.8 Coriolis response to pulsating flow and entrained air at a pump speed of $7.2 \mathrm{~Hz}$.

E.9 Magnetic response to pulsating flow and entrained air at a pump speed of $8.3 \mathrm{~Hz}$.

E.10 Coriolis response to pulsating flow and entrained air at a pump speed of $8.3 \mathrm{~Hz}$.

E.11 Magnetic response to pulsating flow and entrained air at a pump speed of $9.5 \mathrm{~Hz}$

E.12 Coriolis response to pulsating flow and entrained air at a pump speed of $9.5 \mathrm{~Hz}$.

E.13 Magnetic response to pulsating flow and entrained air at a pump speed of $10.7 \mathrm{~Hz}$.

E.14 Coriolis response to pulsating flow and entrained air at a pump speed of $10.7 \mathrm{~Hz}$.

E.15 Magnetic response to pulsating flow and entrained air at a pump speed of $11.9 \mathrm{~Hz}$.

E.16 Coriolis response to pulsating flow and entrained air at a pump speed of $11.9 \mathrm{~Hz}$ 



\section{ACRONYMS AND ABBREVIATED TERMINOLOGY}

$\begin{array}{ll}\text { ADU } & \text { ammonium diuranate } \\ \text { AUC } & \text { ammonium uranyl carbonate } \\ \text { AVLIS } & \text { atomic vapor laser isotope separation } \\ \text { DOE } & \text { U.S. Department of Energy } \\ \text { EMIS } & \text { electromagnetic isotope separation } \\ \text { FOM } & \text { figure of merit } \\ \text { GCP } & \text { gas centrifuge enrichment plant } \\ \text { GDP } & \text { gaseous diffusion enrichment plant } \\ \text { IAEA } & \text { International Atomic Energy Agency } \\ \text { MSE } & \text { mean-squared error } \\ \text { MTU } & \text { metric tons of uranium } \\ \text { MTU/yr } & \text { metric tons of uranium per year } \\ \text { NUCP } & \text { natural uranium conversion plant } \\ \text { ORNL } & \text { Oak Ridge National Laboratory } \\ \text { SQ } & \text { significant quantity } \\ \text { TBP } & \text { tributyl phosphate } \\ \text { UF }_{4} & \text { uranium tetrafluoride } \\ \text { UF }_{6} & \text { uranium hexafluoride } \\ \text { UO }_{2} & \text { uranium dioxide } \\ \text { UOC } & \text { uranium ore concentrate }\end{array}$





\section{EXECUTIVE SUMMARY}

The objectives of the work discussed in this report were to

- develop a flow loop that would simulate the purified uranium-bearing aqueous stream exiting the solvent extraction process in a natural uranium conversion plant (NUCP),

- develop a test plan that would simulate normal operation and disturbances that could be anticipated in an NUCP,

- use the flow loop to test commercially available flowmeters for use as safeguards monitors, and

- recommend a flowmeter for production-scale testing at an NUCP.

This report includes a discussion of current International Atomic Energy Agency safeguards as they apply to NUCPs, a summary of uranium conversion methods, and a review of potential places for diversion within an NUCP.

The uranyl nitrate flow loop was developed to simulate the purified uranium-bearing aqueous stream exiting the solvent extraction process in a small NUCP (100 metric tons of uranium per year). This flow loop was used to test various commercially available flowmeters for use as safeguards monitors in actual NUCPs and to recommend the meter of choice for field testing at a productionscale facility. The flow loop was designed to measure the response of flowmeters to various flow rates $(0-11 \mathrm{~L} / \mathrm{min})$, entrained air, pulsating flow, and entrained organic (1-5 vol \% $n$-dodecane).

A discussion of flowmeter types (vortex shedding, turbine, magnetic, ultrasonic, and Coriolis) is provided, with a listing of the advantages and disadvantages for each type. Three instruments were selected for testing in the flow loop at Oak Ridge National Laboratory: the Endress+Hauser Promass 83F Coriolis flowmeter, the Yokogawa ADMAG SE magnetic flowmeter, and the Controlotron 1010N1 transit-time ultrasonic flowmeter. The Endress+Hauser Promass 83F Coriolis meter was selected because of its ability to measure density as well as mass and volumetric flow rates with excellent accuracy and repeatability. The Coriolis meter can also measure temperature. The Yokogawa ADMAG SE magnetic flowmeter was selected because of its level of accuracy and ability to measure low flow rates. The Controlotron 1010N1 clamp-on transit-time ultrasonic meter was selected because of its nonintrusive nature.

The instruments were calibrated first using water and then using uranyl nitrate solution. A series of experiments was conducted to determine the most effective flowmeter for measuring the flow rate of uranyl nitrate solution in a pipe. From the initial testing, it was determined that the ultrasonic meter was inadequate for this application. The ultrasonic flowmeter required that all the fluid properties be specified and that the properties remain unchanged, which would not be reasonable in an NUCP. The two remaining candidate meters, the Coriolis and the magnetic, were evaluated according to specific selection criteria:

- general industrial applicability and manufacturing quality,

- whether the meter had a pulse output,

- whether the meter had both volume and mass measurement outputs,

- meter specifications and applicability to the process conditions/environment, and

- measurement error of the meter in this application (residual nonlinearity in response).

Both of the meters were found to satisfy generally accepted industrial standards for quality and robustness, and both are sufficiently well engineered that either could properly function in a nuclear facility environment. Based on process specifics, a slight preference existed for the Coriolis meter because of overall size and accuracy of measurement and a slight preference existed for the magnetic meter because of measurement range. Based on the testing results, however, the Coriolis meter was selected for production-scale testing. 
The following conclusions were made based on the results of testing of the uranyl nitrate flow loop:

- The Coriolis flowmeter and the magnetic flowmeter were found to perform accurately under the conditions expected at an NUCP.

- The ultrasonic flowmeter was not appropriate for the operating conditions anticipated at an NUCP.

- Organic contamination tests were not completed due to a second pump failure, possibly because of an unexpected incompatibility of gear material with $n$-dodecane or the combination of $n$-dodecane and uranyl nitrate. The first pump failure occurred during testing prior to the addition of organic and was most likely due to an incompatibility of gear material with uranyl nitrate.

- The zero-drift for the Coriolis meter and the magnetic meter was negligible over $8 \mathrm{~h}$ of continuous testing.

- The flow response to the possible range of flow rates was linear, as would be expected with a positive displacement pump.

- Air entrainment resulted in a decreased measurement in flow rate (both volumetric and mass) for both the Coriolis and the magnetic flowmeters. This finding is expected because the instruments are not designed to measure gas flows, including entrained air. Therefore, because less liquid mass/volume was moving through the system, the flow rate was decreased.

- The pulsating flow tests were intended to demonstrate the instrument performance during start-up and/or shutdown. As the pump was cycled on and off, the flow rate readings would drop below zero when the pump was turned off and never return to the calibrated flow rate during the time the pump was running. In an actual plant situation, it would be necessary to determine how long it takes the instrument to stabilize after the process is started.

- The tests that included both pulsating flow and entrained air represented a worst-case scenario, which is not expected under normal conditions at a production facility. 


\section{INTRODUCTION}

The objectives of the work discussed in this report were to

- develop a flow loop that would simulate the purified uranium-bearing aqueous stream exiting the solvent extraction process in a natural uranium conversion plant (NUCP),

- develop a test plan that would simulate normal operation and disturbances that could be anticipated in an NUCP,

- use the flow loop to test commercially available flowmeters for use as safeguards monitors, and

- recommend a flowmeter for production-scale testing at an NUCP.

There has been interest in safeguarding conversion plants because the intermediate products [uranium dioxide $\left(\mathrm{UO}_{2}\right)$, uranium tetrafluoride $\left(\mathrm{UF}_{4}\right)$, and uranium hexafluoride $\left(\mathrm{UF}_{6}\right)$ ] are all suitable uranium feedstocks for producing special nuclear materials. Furthermore, if safeguards are not applied virtually any nuclear weapons program can obtain these feedstocks without detection by the International Atomic Energy Agency (IAEA). Historically, IAEA had not implemented safeguards until the purified $\mathrm{UF}_{6}$ product was declared as feedstock for enrichment plants. ${ }^{1} \mathrm{H}$. A. Elayat et al. provide a basic definition of a safeguards system: "The function of a safeguards system on a chemical conversion plant is in general terms to verify that no useful nuclear material is being diverted to use in a nuclear weapons program." ${ }^{2}$ The IAEA now considers all highly purified uranium compounds as candidates for safeguarding. ${ }^{3}$

DOE is currently interested in "developing instruments, tools, strategies, and methods that could be of use to the IAEA in the application of safeguards" for materials found in the front end of the nuclear fuel cycle - prior to the production of the uranium hexafluoride or oxides that have been the traditional starting point for IAEA safeguards. ${ }^{4,5}$ Several national laboratories, including Oak Ridge, Los Alamos, Lawrence Livermore, and Brookhaven, have been involved in developing tools or techniques for safeguarding conversion plants. This study was sponsored by the U.S. Department of Energy (DOE) NA-241, Office of Dismantlement and Transparency.

\subsection{CURRENT IAEA SAFEGUARDS}

Before 2003, the IAEA had not considered conversion plants in developing safeguards policies. However, IAEA now considers all highly purified uranium compounds as candidates for safeguarding: this increased scope includes the intermediate products of an NUCP. ${ }^{3}$ The following provides an overview of the current safeguards policy for conversion plants.

Operating in accordance with international agreements, the IAEA has had limited ability to monitor the front end of the nuclear fuel cycle, because safeguards programs are currently constrained to control nuclear materials at the start of the uranium enrichment processes, with $\mathrm{UF}_{6}$ as its feedstock and designated as the chemical form of interest. ${ }^{6}$ Since the final product of an NUCP is $\mathrm{UF}_{6}$, IAEA accountability data begin at the final process step of the NUCP when the product is declared. Without information to ascertain a uranium mass balance between the yellowcake feedstock and the $\mathrm{UF}_{6}$ final

product, there are no assurances that the declared $\mathrm{UF}_{6}$ product accurately represents the total uranium produced by an NUCP. ${ }^{6}$

J. Doo et al. outline a new approach developed by the IAEA for safeguards at NUCPs. ${ }^{3}$ Current IAEA policy, as stated in paragraph 34(c) of INFCIRC/153 (corrected), considers any purified aqueous uranium solution or any purified uranium oxides to be nuclear material of a composition and 
purity suitable for isotopic enrichment or fuel fabrication, respectively, and therefore subject to full safeguards procedures. ${ }^{7}$

When any nuclear material of a composition and purity suitable for fuel fabrication or for being isotopically enriched leaves the plant or the process stage in which it has been produced, or when such nuclear material, or any other nuclear material produced at a later stage in the nuclear fuel cycle, is imported into the State, the nuclear material shall become subject to the other safeguards procedures specified in the Agreement.

However, in the past, the IAEA had not been consistent with the technical interpretation of this requirement or the implementation of safeguards at NUCPs. The revised policy "requires that full safeguards procedures should be applied no later than the first point in the conversion process at which such material leaves the process stage or the plant in which it is produced." 3 The objective of the DOE-sponsored studies is to reduce the probability of uranium ore concentrate (UOC) being converted to a form suitable for use in the production of undeclared special nuclear materials. ${ }^{6}$ Although a simple accounting system for assessing uranium inputs and outputs will provide some assurance that undeclared material is not leaving an NUCP, the use of unattended monitoring instruments can validate accountability data and significantly improve a safeguards program. ${ }^{6} \mathrm{~B}$. Boyer et al. stated that "the goal of IAEA safeguards is the timely detection of a diversion of a significant quantity (SQ) of nuclear material." "4 The IAEA has declared that 10 metric tons of uranium (MTU) is an SQ for facilities that process only natural uranium, with a timeliness period of 1 year and a detection probability of $50 \%$ (ref. 4 ).

An overall safeguards approach proposed by B. Boyer et al. requires the plant operator to make a declaration of the material balance for the plant and the IAEA to verify that declaration. ${ }^{4}$ The IAEA verification measures include the confirmation of plant design information, auditing of records and reports, and independent measurement of a portion of the nuclear materials that comprise the flows and inventories that constitute the declared material balance. The verification of the declared material balance is important in cases in which the plant does not have access to undeclared feed materials. In such circumstances, the plant can acquire the desired material only by diversion from the declared material balance. ${ }^{4}$

B. Boyer et al. stated that "verification of the declared material balance alone in general will not detect the processing of undeclared feed to produce undeclared product, unless the operator inadvertently reveals the existence of the undeclared materials in his declarations or fails to conceal the undeclared operations." "Therefore, additional safeguards measures are required to detect any undeclared processing. Traditional IAEA methods (e.g., application of containment and surveillance measures) could provide some detection capability. The IAEA has revised its approach to NUCP safeguards to include more short-notice random inspections during the year to gain more flexibility and unpredictability in conducting inspections. The IAEA has made increasing use of unattended monitoring to verify operations at safeguarded facilities, which could offer an effective method for detecting the processing of undeclared materials in an NUCP. ${ }^{4}$

In an ideal situation, safeguards would involve the continuous presence of IAEA inspectors at the plant and the installation of unattended monitors on each of the process vessels. However, such intensive and intrusive approaches are not feasible because of cost, shortage of IAEA manpower, difficulty in operator acceptance, and existing political constraints. ${ }^{4}$ A safeguards approach capable of detecting the processing of undeclared materials in a small NUCP might include the following components ${ }^{4}$ :

- IAEA unattended monitoring instruments to measure the uranium content of intermediate process flow streams at key points in the process, primarily to detect the processing of undeclared feed; 
- IAEA verification of the declared uranium balance, primarily to detect the diversion of declared uranium; and

- operator declarations of specified nuclear material quantities and operating parameters on a daily basis.

\subsection{CONVERSION METHODS}

Conversion plants purify the uranium ore from the mining and milling operation of the nuclear fuel cycle; the purified uranium is also converted to a gaseous form at the conversion plant prior to shipment to the enrichment plant. Conversion plants are located worldwide, producing $\sim 36,000 \mathrm{MT}$ of natural uranium per year, which supports 441 nuclear power plants. ${ }^{6}$ Conversion plants can be classified into three categories based on production capacity in metric tons of uranium per year (MTU/yr): small (100 MTU/yr), medium (1000 MTU/yr), or large (10,000 MTU/yr). These plants use some variation of the two processes discussed in this section. The work discussed in this report will focus specifically on a 100-MTU/yr NUCP using the wet solvent extraction process.

In order to develop a means of testing instruments in conditions that simulate an NUCP, it was necessary to review common conversion methods used in various NUCPs. Moghissi et al. state that conversion is required because "uranium concentrate from milling operations is free of the bulk of the ore components, but is still far from pure enough for use in fuel." ${ }^{8}$ The UOC contains the equivalent of $75-80 \% \mathrm{U}_{3} \mathrm{O}_{8}$. The UOC becomes the feed material to a conversion plant. At the conversion plant the UOC is submitted to a series of operations that remove impurities and eventually convert the $\mathrm{U}_{3} \mathrm{O}_{8}$ to gaseous $\mathrm{UF}_{6}$, which is then in the necessary form for subsequent isotopic enrichment by the gaseous diffusion or gas centrifuge process. ${ }^{8}$ The two methods (dry hydrofluor process or wet solvent extraction process) described below are used commercially for this conversion.

The first is a dry method, called the dry hydrofluor process, which directly processes the concentrates in a series of fluidized-bed reactors, followed by fractional distillation. The dry hydrofluor process eliminates impurities either as volatile compounds or as solid constituents of ash. In the dry process, the volatile fluorides are separated from the uranium product by taking advantage of relative differences in volatility and, after treatments, the impurities leave the process as solid waste. ${ }^{8}$ The dry hydrofluor process consists of the following operations:

1. Preprocess handling, weighing, sampling, and storage.

2. Roasting and fluidized-bed reduction of the $\mathrm{U}_{3} \mathrm{O}_{8}$ to $\mathrm{UO}_{2}$ using hydrogen from cracked ammonia.

3. Fluidized-bed hydrofluorination of the $\mathrm{UO}_{2}$ using anhydrous $\mathrm{HF}$ to form a crude $\mathrm{UF}_{4}$.

4. Fluidized-bed fluorination of the $\mathrm{UF}_{4}$ using elemental fluorine to make a crude $\mathrm{UF}_{6}$.

5. Fractional distillation to refine the $\mathrm{UF}_{6}$. The distillation step removes volatile fluorides generated in the fluorination step, predominantly those of molybdenum and vanadium. ${ }^{8}$

The second method is a wet process that uses solvent extraction to purify the $\mathrm{U}_{3} \mathrm{O}_{8}$ feed material before its conversion to $\mathrm{UF}_{6}$. This method separates impurities by preferentially extracting the uranium into an organic solvent, leaving other constituents in the aqueous phase. The method consists of the following operations:

1. preprocess handling, weighing, sampling, and storage;

2. digestion in hot nitric acid;

3. countercurrent solvent extraction with tributyl phosphate (TBP) in kerosene;

4. stripping of uranium from the organic phase as uranyl nitrate solution $\left[\mathrm{UO}_{2}\left(\mathrm{NO}_{3}\right)_{2}\right]$;

5. calcination to $\mathrm{UO}_{3}$;

6. fluidized-bed reduction to $\mathrm{UO}_{2}$ using hydrogen from cracked ammonia;

7. fluidized-bed hydrofluorination in a two-stage countercurrent reactor to form $\mathrm{UF}_{4}$ using anhydrous HF; and 
8. flame reactor fluorination to $\mathrm{UF}_{6}$ by reaction with fluorine gas. ${ }^{8}$

The conversion method employed by an NUCP is based on the size of the plant. For most plants, a generic production process for natural uranium conversion using the wet method begins with yellowcake dissolution with nitric acid, followed by purification using solvent extraction techniques, and then evaporation to produce a concentrated, purified uranyl nitrate solution. For small plants, ammonia or ammonium hydroxide and carbon dioxide are used to convert uranyl nitrate to a precipitate of ammonium diuranate (ADU) or ammonium uranyl carbonate (AUC), respectively. After precipitation, calcination in the presence of hydrogen produces $\mathrm{UO}_{2}$ powder. Precipitation processes can be operated in a continuous mode but are well suited to batch production techniques typical of small-scale plants. Medium and large plants typically utilize a denitration process, which is more commonly used in continuous operations where higher production capabilities are required. The denitration process uses heat to dehydrate and denitrate the purified uranyl nitrate solution and produce $\mathrm{UO}_{3}$, followed by oxide reduction with hydrogen to produce $\mathrm{UO}_{2}$. Then, irrespective of the size of the plant, the $\mathrm{UO}_{2}$ is hydrofluorinated to $\mathrm{UF}_{4}$ using $\mathrm{HF}$. The $\mathrm{UF}_{4}$ can then be fluorinated into $\mathrm{UF}_{6}$ using $\mathrm{F}_{2}$ or reduced into uranium metal using magnesium or calcium and heat. ${ }^{5}$

Figure 1 shows a simplified flow diagram of the conversion process; the left side represents the processing steps most frequently used in small-scale chemical conversion plants. In plants of all sizes that use this method, yellowcake is dissolved in acid, purified using solvent extraction, and concentrated by evaporation. In a small plant, the concentrated uranyl nitrate solution will be precipitated and calcined to produce $\mathrm{UO}_{2}$. In medium or large plants (right side of figure), $\mathrm{UO}_{2}$ will be produced using denitration and oxide reduction processes. The $\mathrm{UO}_{2}$ will then be hydrofluorinated to $\mathrm{UF}_{4}$. In most cases, the $\mathrm{UF}_{4}$ is fluorinated to $\mathrm{UF}_{6}$. However, the $\mathrm{UF}_{4}$ can also be reduced to uranium metal for atomic vapor laser isotope separation (AVLIS) enrichment. In addition, clandestinely produced $\mathrm{UF}_{6}$ (green arrow) could be fed into the process at the precipitation step or $\mathrm{UO}_{2}$ could be diverted for reactor fuel production (as shown in Fig. 1).

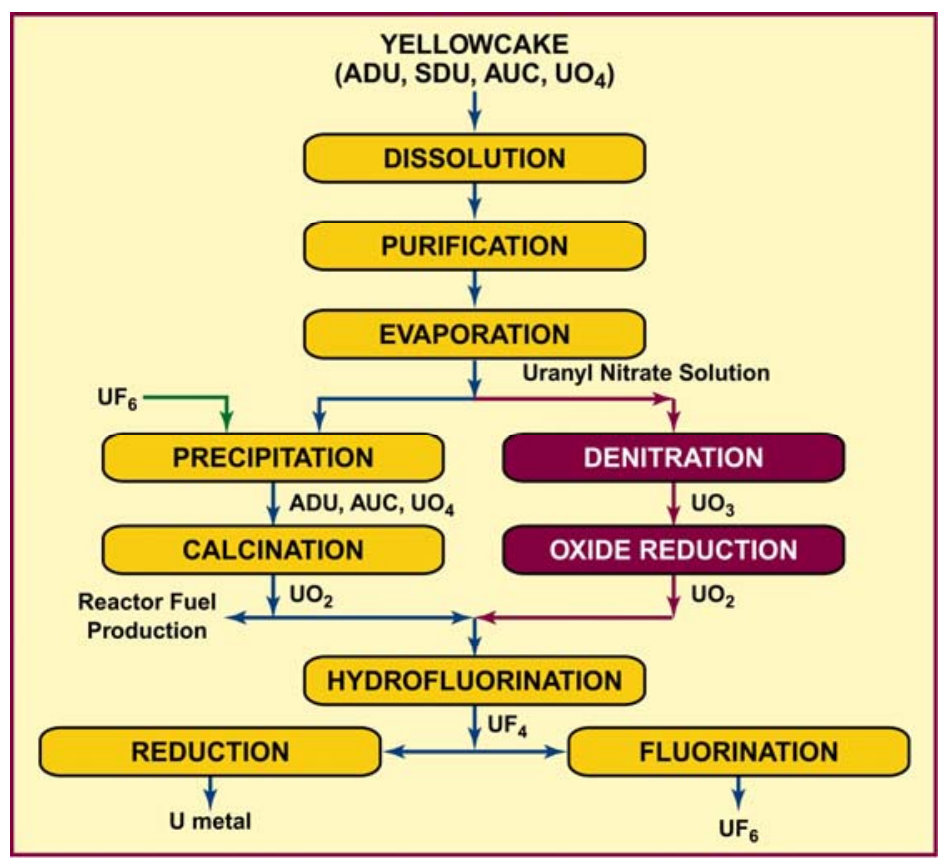

Fig. 1. Simplified flow diagram of the wet solvent extraction process used to convert yellowcake to $\mathbf{U F}_{6}$ or uranium metal. Note that the left side represents the processing steps most frequently used in smallscale chemical conversion plants. [Source: R. L. Faulkner, J. M. Begovich, J. J. Ferrada, R. D. Spence, J. M. Whitaker, W. J. Bicha, and L. G. Loden, "Oak Ridge Efforts to Enhance Conversion Plant Safeguards," Proceedings 45th Annual Meeting of Institute of Nuclear Materials Management (INMM), July 18-22, 2004, Orlando, Fla.] 


\subsection{POTENTIAL PLACES FOR DIVERSION}

The intermediate products of an NUCP [uranium dioxide $\left(\mathrm{UO}_{2}\right)$, uranium tetrafluoride $\left(\mathrm{UF}_{4}\right)$, and unpurified $\mathrm{UF}_{6}$ ] are all suitable uranium feedstocks for special nuclear materials production and could supply virtually any nuclear weapons program without triggering IAEA detection. ${ }^{6} \mathrm{R}$. Faulkner et al. also state that "due to these considerations and the consequences for inadequate control of special nuclear materials, it is reasonable and prudent to include an NUCP in an IAEA safeguards monitoring program." ${ }^{6}$ B. Boyer et al. determined that the two principal safeguards concerns at an NUCP are as follows: "1) diversion of pure materials for further processing or use elsewhere and 2) processing of undeclared feed to produce undeclared pure products (e.g., $\mathrm{UO}_{2}, \mathrm{UF}_{6}$ )" (ref. 4).

According to R. L. Faulkner et al., "a clear understanding of the chemical conversion process is essential in selecting an appropriate set of safeguard controls." However, traditional IAEA inventory controls do not begin until the last step in an NUCP, when the $\mathrm{UF}_{6}$ product is certified for use as feed to an enrichment facility. R. L. Faulkner et al. state the reasoning behind safeguarding NUCPs: "Each NUCP processing step increases the nuclear material attractiveness for diversion as the uranium is purified and as the chemical form is converted to one that is more suitable for use in a nuclear weapon[s] program. The quantity of uranium available, the ease of removal from the process, and the ability to obfuscate diversion were special considerations in the diversion analysis. Safeguard controls must include defense-in-depth approaches to achieve a reasonable level of detection capability."

R. Faulkner et al. performed a diversion analysis by analyzing the generic conversion process to determine potential diversion routes for intermediate products. ${ }^{6}$ After the uranium is in the form of a purified uranyl nitrate solution, it becomes attractive for use in a weapons complex. The purified uranyl nitrate solution can be denitrated to form $\mathrm{UO}_{3}$ or $\mathrm{UO}_{2}$. Either of these intermediate products is well suited for production of $\mathrm{UCl}_{4}$ by chlorination, which is the preferred chemical form for an electromagnetic isotope separation (EMIS) enrichment process or for a chemical/ion-exchange enrichment process. Also, $\mathrm{UO}_{2}$ can be hydrofluorinated to produce $\mathrm{UF}_{4}$, which can be metallothermically reduced to uranium metal for use in an AVLIS enrichment process or in plutonium production. Additionally, uncertified $\mathrm{UF}_{6}$ is not currently monitored by IAEA safeguards and could be shipped to a clandestine site for fractional distillation prior to feeding to a gas centrifuge enrichment plant (GCP) or gaseous diffusion enrichment plant (GDP).

Diversion scenarios also include other options, such as the following ${ }^{6}$ :

- material substitution (substitution of feed materials that have higher uranium content than declared, or the clandestine removal of uranium-bearing material in exchange for materials of similar characteristics but with less or no uranium content);

- equipment alternations [addition of bypass piping, valving, or other equipment used to divert or introduce materials clandestinely; or modification to equipment (e.g., heating), resulting in incomplete conversions or inefficient operation with excess uranium in the waste or tails];

- modified operations (intentionally operating processes inefficiently so that more uranium is contained in recycle, sample, or waste streams); and

- data tampering (keeping two records of operation with one showing less throughput than actually processed; declaration of understated records to inspectors).

Figure 2 shows the potential diversion paths for intermediate products from an NUCP. 


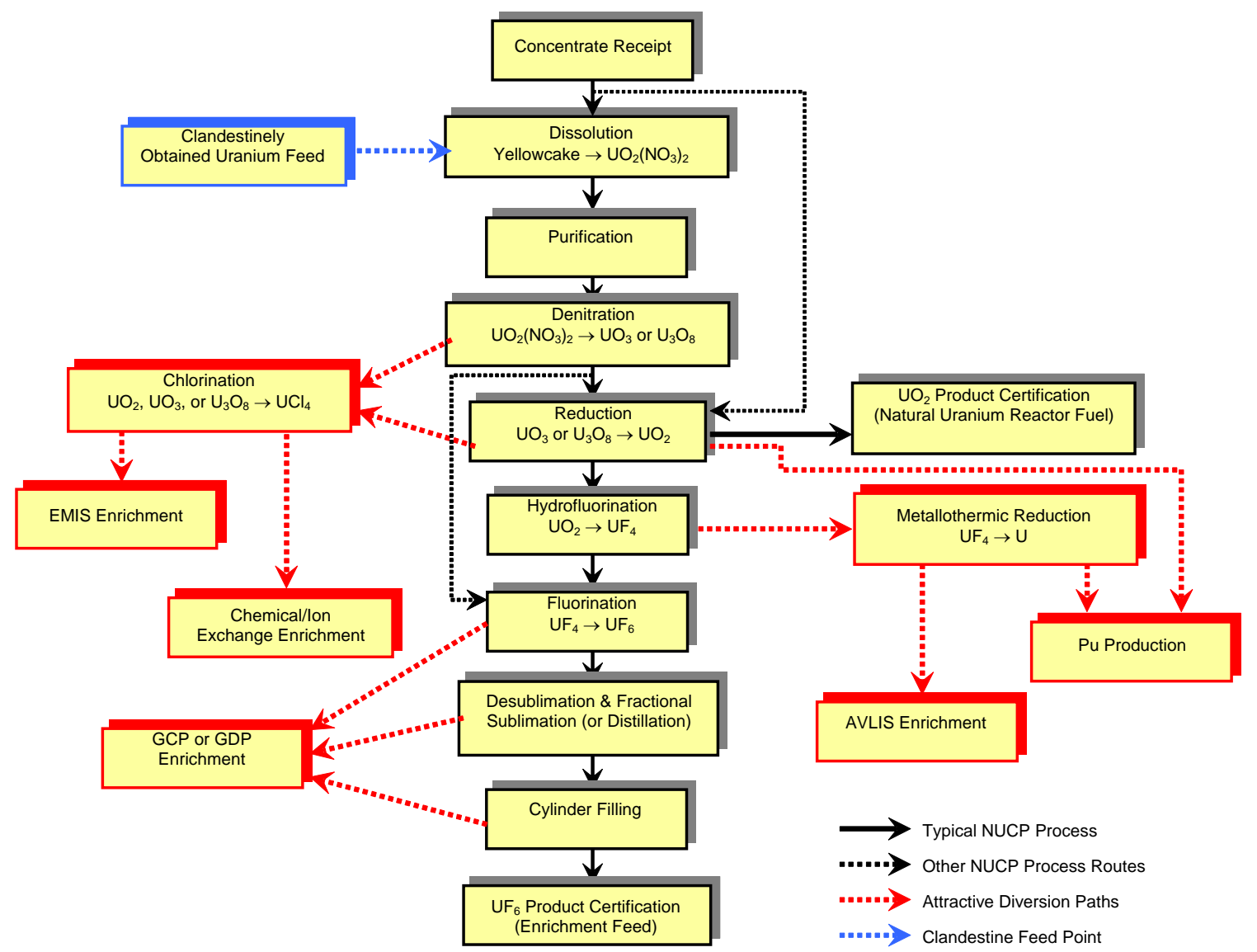

Fig. 2. Potential diversion paths for intermediate products from an NUCP. [Source: R. L. Faulkner, J. M. Begovich, J. J. Ferrada, R. D. Spence, J. M. Whitaker, W. J. Bicha, and L. G. Loden, "Oak Ridge Efforts to Enhance Conversion Plant Safeguards," Proceedings 45th Annual Meeting of Institute of Nuclear Materials Management (INMM), July 18-22, 2004, Orlando, Fla.]

A safeguards system that uses a combination of accountability principles with unattended monitors to verify data would significantly enhance a diversion detection system. R. L. Faulkner et al. have proposed that "the optimum system would include verifiable accountability data for feed and the withdrawal streams," as well as the presence of multiple in-line detection systems. ${ }^{5}$ As shown in Fig. 3 , these researchers identify eight points in an overall uranium conversion process for possible monitoring or accountability. The first monitoring point is the accountability data for the declared yellowcake receipt at the plant inlet (Point 1). A comparison of the record of the rate and amount of uranium mass exiting the plant in the $\mathrm{UF}_{6}$ product (Point 8) with that of a record of the uranium mass entering the plant in the feed provides the means for an overall plant uranium mass balance, minus the uranium leaving as waste. The yellowcake exiting the feed hopper into the dissolver (Point 2) is the first opportunity in the process to verify the uranium mass recorded in the accountability data for the yellowcake received at the NUCP. One possible location for in-line monitoring is at the point just after dissolution, downstream of the uranyl nitrate tank (Point 3). This would be the first point at which uranium is in solution. Both streams exiting the strip column should be monitored: the aqueous stripping solution loaded with uranium (Point 4) and the organic stream stripped of uranium (Point 5). These points help to prevent inefficient stripping by the operator and provide a mass check after the purification of the uranium. The concentrated solution from the evaporator can be measured at one of two locations: (1) just out of the evaporator before the pump (Point 6a) or (2) downstream of the reflux lines and valves but before the cooler (Point $6 \mathrm{~b}$ ). Point 7, the last in-line process monitor, would be located at the end of the precipitation or denitration step. Point 7 also helps to verify the 
amount of uranium dissolved and purified. With two accountability points and six in-line monitors in place, it is possible that this defense-in-depth safeguards approach could detect a significant quantity of uranium diversion with a reasonably high probability of detection for the small-scale plant. A minimum case for the small-scale (100-MTU/yr) plant is probably one in-line monitor, preferably at Point 4, plus the accountability data at Points 1 and 8 (ref. 5).

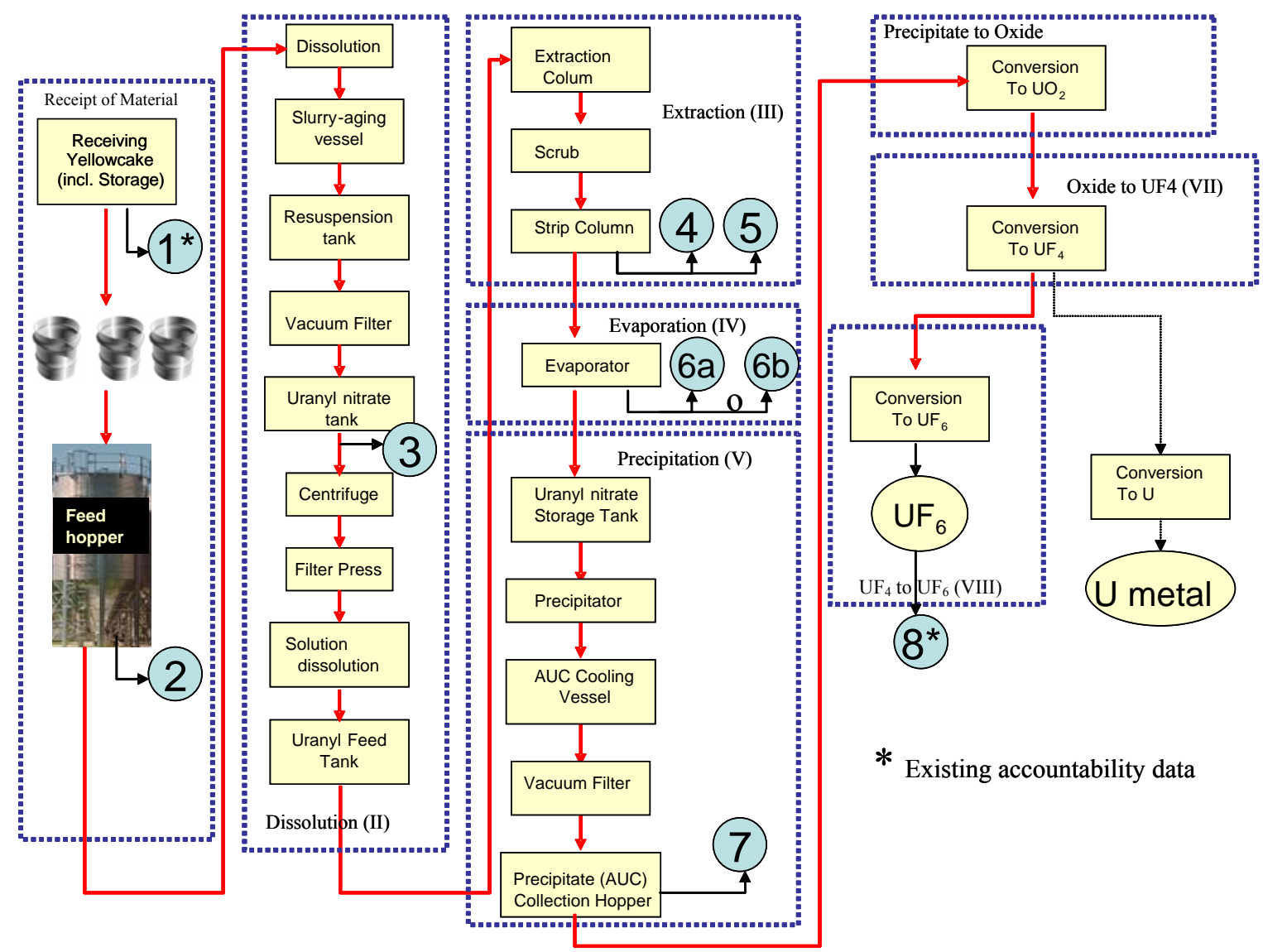

Fig. 3. Uranium conversion process for a small-size plant with proposed safeguards monitoring points identified. Note that Points 1 and 8 represent existing accountability data and that Point 8 is also the traditional start of IAEA safeguards. The remaining points are proposed in-line process monitoring points; values at all eight points are correlated through material balance constraints and process conditions. [Source: R. L. Faulkner, J. M. Begovich, J. J. Ferrada, R. D. Spence, J. M. Whitaker, W. J. Bicha, and L. G. Loden, "Oak Ridge Efforts to Enhance Conversion Plant Safeguards," Proceedings 45th Annual Meeting of Institute of Nuclear Materials Management (INMM), July 18-22, 2004, Orlando, Fla.]

R. Faulkner et al. provide the following information on possible monitors that could be used at the points shown in Fig. 3 (ref. 6). These monitoring points are driven mainly by mass balance principles. Therefore, the monitoring points should be supported by instrumentation that can verify mass balances. Flowmeters, to measure flow of material, and instruments that measure concentration of uranium are ultimately required for this analysis. Real-time in-line monitoring may be practical for a uranium solution, after the uranium is extracted from the yellowcake and before the uranium is changed into a solid again (Fig. 3, Points 3-6). This type of monitoring requires the liquid flow rate (typically volume per unit time) and uranium concentration (mass per unit volume). Volumetric flowmeters are readily available from commercial suppliers. Mass flowmeters are also available, many of which also measure density. Monitoring of liquid density can help verify the uranium concentration because density is a function of the concentration. R. Faulkner et al. note that the 
primary monitoring should be direct measurement of the uranium concentration and flow rate and that monitoring of density should be secondary to verify that the primary monitoring is working properly. ${ }^{6}$ In-line meters for direct measurement of uranium concentration are more problematic. Low uranium concentrations result in impractical count times for the low-energy gamma emissions from radiodecay daughters. Photometric meters for in-line measurement of uranium concentration exist, but the process of matching safeguard needs with instrument capabilities has not been done. Selecting monitoring points where higher concentrations are expected (e.g., after evaporators) would be helpful. For a solid material stream, in-line monitoring of uranium is impractical. Gravimetric feeding of solids accompanied by real-time recording of the mass fed per unit time is possible. However, it is necessary to know the uranium assay of the yellowcake being fed into the dissolver in order to obtain the uranium mass fed into the dissolver per unit time. In order to determine the uranium assay, grab samples would have to be taken at regular intervals followed by destructive analysis of the uranium concentration. Gamma spectroscopy of the low-energy gamma from the uranium decay daughters is being used with good success for qualitative, semiquantitative, and even quantitative analysis, especially with regard to the relative ratio of ${ }^{235} \mathrm{U}$ and ${ }^{238} \mathrm{U}$. However, it is questionable whether this technique or others will provide practical real-time monitoring of the uranium concentration in a process flow of a solid material with the precision and accuracy required for detecting undeclared production. A gravimetric feed and monitoring system may provide the total mass per unit time, but grab sampling and analysis would still be required to obtain the uranium mass per unit time. In addition, the accountability records of the facility (e.g., number of drums and their weights, with the date and time filled) can be verified using on-site monitoring (e.g., cameras), random checking of drum weight, and a sampling program for the uranium concentration. No matter the location in the plant, "real-time process monitors that can compare NUCP accountability data with actual process data can be very useful, especially if the software can indicate trends in the relationship of accountability and actual data using statistical control charts to document anomalies."

\section{DESIGN AND FLOWMETER SELECTION}

\subsection{DESIGN}

One objective of the work discussed in this report was to design and construct a small-scale uranyl nitrate flow loop to simulate the purified uranium-bearing aqueous stream exiting the solvent extraction process in a small NUCP (Point 4, Fig. 3). This flow loop was used to test various commercially available flowmeters for use as safeguards monitors in actual NUCPs and to recommend the meter of choice for field testing at a production-scale facility.

Generally, the aqueous stream exiting the solvent extraction process would have a concentration of $80-100 \mathrm{~g} \mathrm{U} / \mathrm{L}$ and a corresponding density of approximately $1.015 \mathrm{~g} / \mathrm{cm}^{3}$ (ref. 6). Assuming the plant produced $100 \mathrm{MTU} / \mathrm{yr}$ and operated continuously, the flow rate would be approximately $142 \mathrm{~L} / \mathrm{h}$. However, if the same plant operated $8 \mathrm{~h}$ each day, 5 days per week and 50 weeks each year, the flow rate would increase to approximately $620 \mathrm{~L} / \mathrm{h}$.

The flow loop is fed from one of two tanks: the first contains pure uranyl nitrate ( $80 \mathrm{~g} \mathrm{U} / \mathrm{L})$, and the other contains organic-contaminated uranyl nitrate. The uranium used to make the uranyl nitrate solution was depleted. Each tank can be shut off from the rest of the system. A gear pump is used to pump material through the system, and the motor controller is used to set the flow rate. Temperature and pressure sensors are located upstream and downstream of the flowmeters, and an airline into the system is used for entrained air tests. The flow loop has the capability of testing the responses of the flowmeters to various flow rates $(0-11 \mathrm{~L} / \mathrm{min})$, entrained air, and pulsating flow conditions. Each of the tests can be repeated with entrained organic (1-5 vol $\% n$-dodecane). A simplified diagram of the 
flow loop is shown in Fig. 4. Figures 5 and 6 are photographs of the actual flow loop and the installed instruments, respectively.

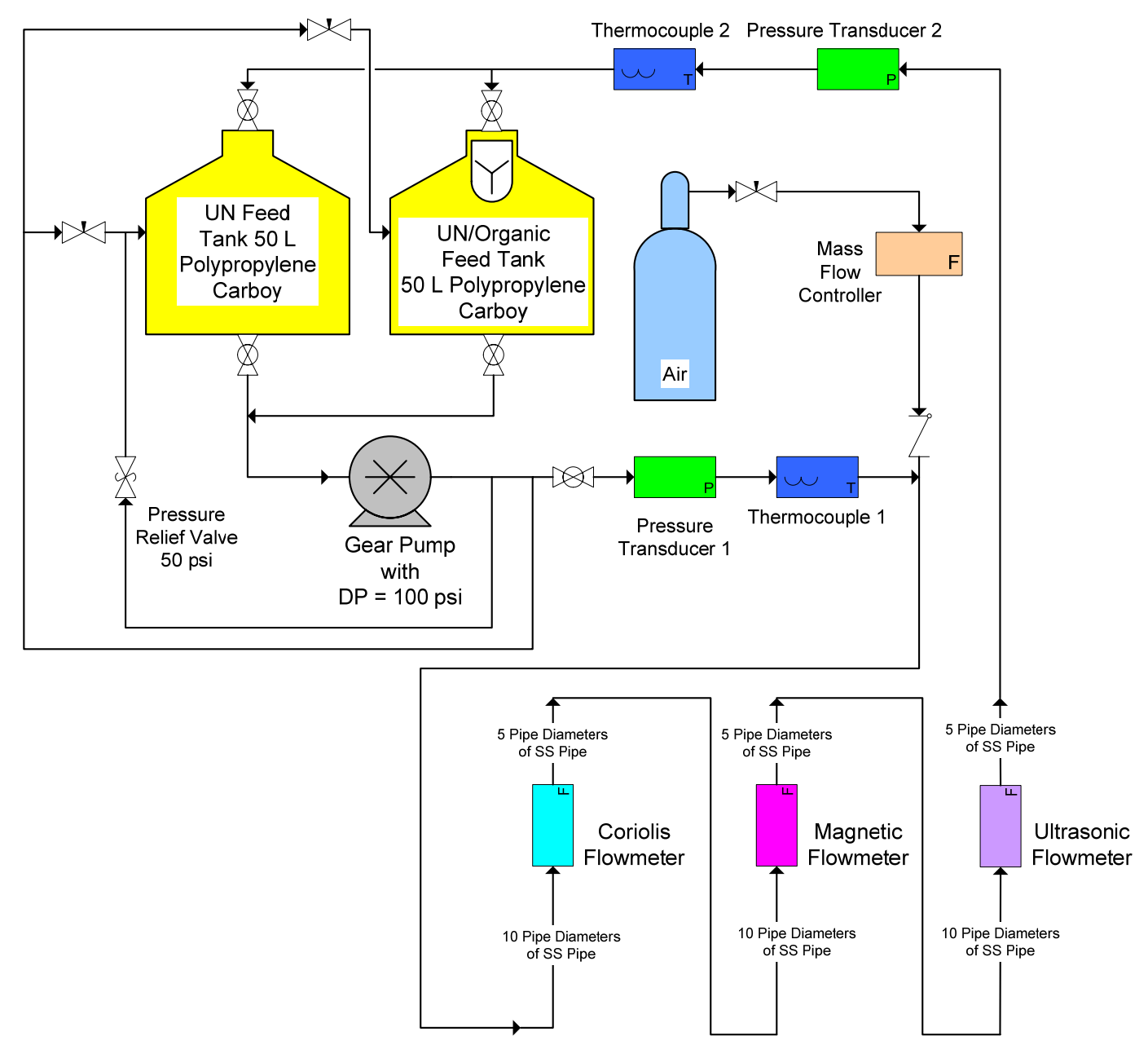

Fig. 4. Simplified diagram of the uranyl nitrate flow loop. The two feed tanks can be used independently: one for pure uranyl nitrate solution and one for organic-contaminated solution. A gear pump is used to pump solution through the system. Pressure and temperature sensors are located upstream and downstream of the flowmeters. Three flowmeters located in series: a Coriolis meter, a magnetic meter, and an ultrasonic meter. Air may also be added to the system upstream of the flowmeters. 


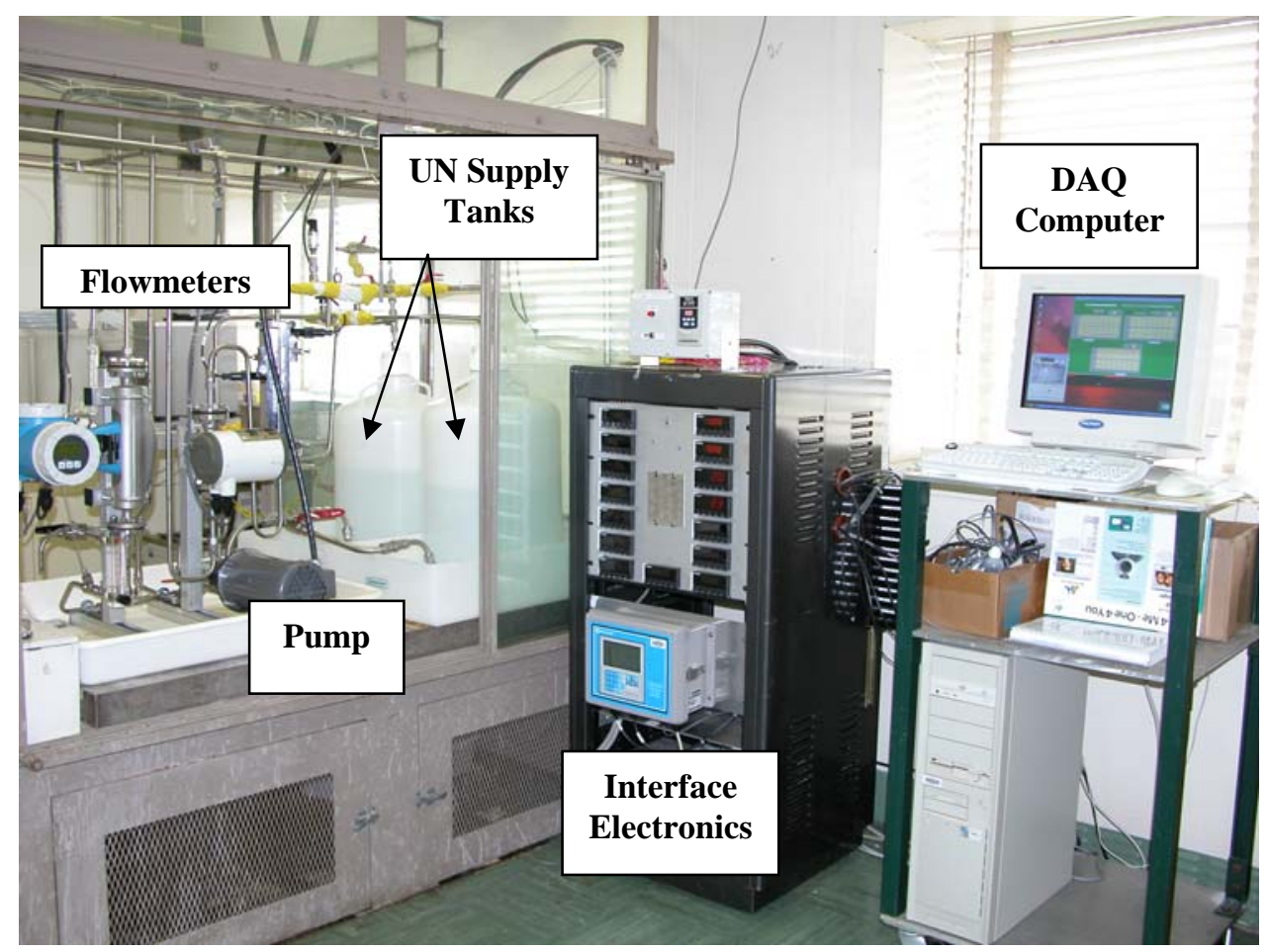

Fig. 5. Complete uranyl nitrate flow loop system. Three flowmeters are located in series. The pump supplies the loop from one of two feed tanks. The electronic components include a computer to collect the data and an interface to transfer data from the instruments to the computer.

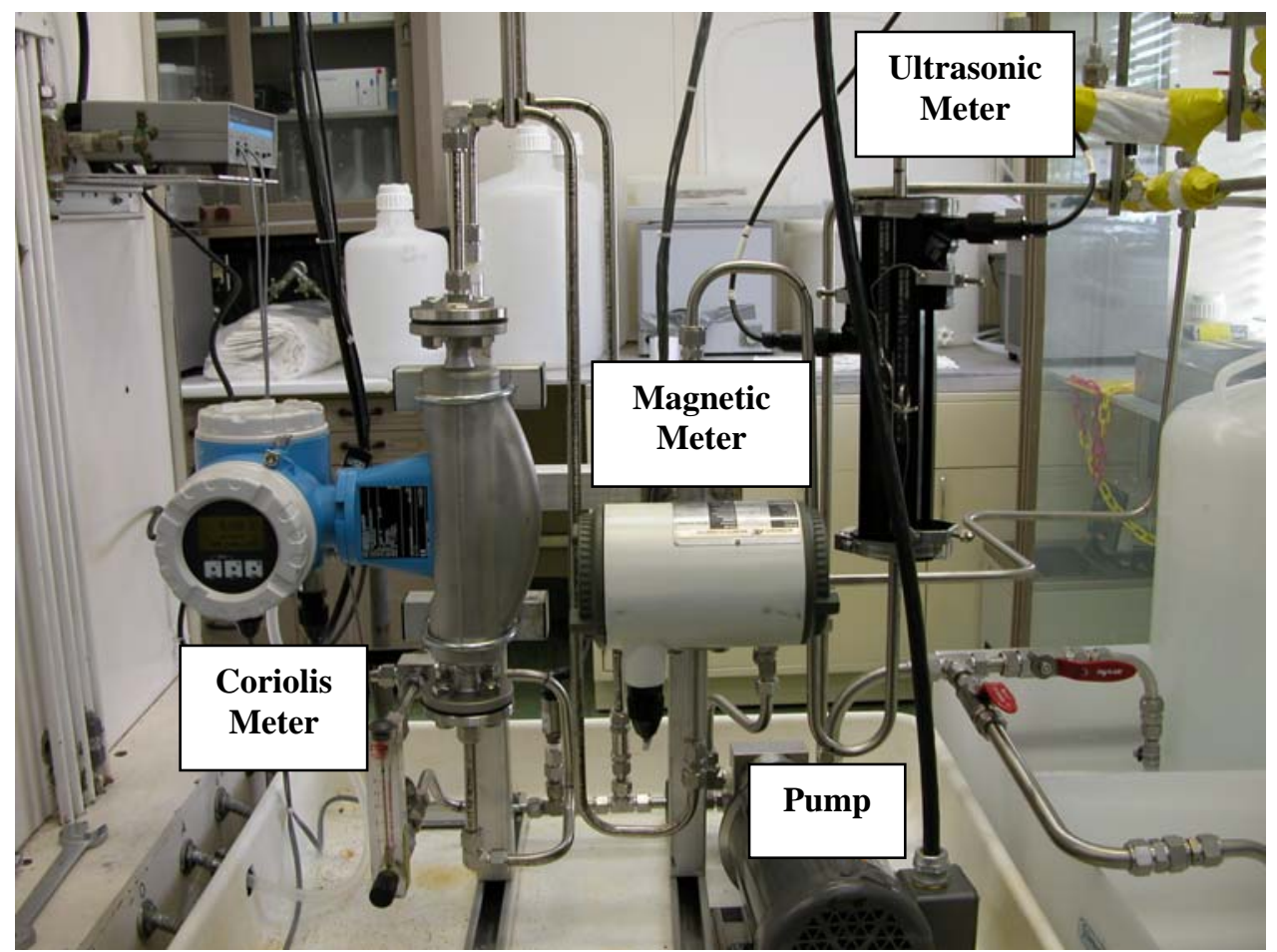

Fig. 6. Close-up view of instruments installed in the uranyl nitrate flow loop. The three instruments (left to right) are as follows: an Endress+Hauser Promass 83F Coriolis flowmeter, a Yokogawa ADMAG AE magnetic flowmeter, and a Controlotron 1010N1 clamp-on ultrasonic flowmeter. 


\subsection{REVIEW OF FLOWMETER TYPES}

Some of the main factors considered when selecting a flowmeter for any application are fluid types, line size, flow measurement range (or linear velocity range), desired accuracy, cost, mounting preference, corrosion resistance, and ease of installation. In this application, flowmeters were also evaluated on the basis of potential intrusiveness to a process. Various types of flowmeters are currently available in the market; each type is based on a different principle. Commonly available flowmeters are vortex shedding, turbine, magnetic, ultrasonic, and Coriolis. Each type is briefly discussed here. More details on flowmeter types are available in Hardy et al., McCabe et al., Perry et al., and Upp and LaNasa. ${ }^{9-12}$

Table 1 summarizes the results of the evaluation of the various types of flowmeters as each applies to the application of safeguards monitors in NUCPs. Each criterion in Table 1 was assigned a subjective rating of Excellent, Good, Fair, or Poor based on information gathered during this study.

Table 1. Summary of the results of flowmeter evaluations ${ }^{\mathrm{a}}$

\begin{tabular}{|c|c|c|c|c|c|c|c|}
\hline \multirow[b]{2}{*}{ Type } & \multicolumn{7}{|c|}{ Evaluation criteria } \\
\hline & $\begin{array}{l}\text { Fluid } \\
\text { types }\end{array}$ & $\begin{array}{c}\text { Line } \\
\text { size }\end{array}$ & $\begin{array}{c}\text { Measurement } \\
\text { range }\end{array}$ & $\begin{array}{c}\text { Desired } \\
\text { accuracy }\end{array}$ & Cost & $\begin{array}{l}\text { Corrosion } \\
\text { resistance }\end{array}$ & $\begin{array}{c}\text { Ease of } \\
\text { installation }\end{array}$ \\
\hline Vortex & $\sigma$ & ? & 0 & 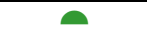 & $\sigma$ & 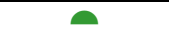 & $\sigma$ \\
\hline Turbine & - & - & - & - & - & อ & - \\
\hline Magnetic & - & อ & อ & ? & C & - & - \\
\hline Ultrasonic & - & $\bullet$ & อ & - & O & - & 0 \\
\hline Coriolis & - & - & - & - & 0 & - & - \\
\hline
\end{tabular}

\subsubsection{Vortex-Shedding Flowmeters}

Vortex-shedding flowmeters operate on the Von Karman effect of flow across a bluff body. This principle states that the flow will alternately shed vortices from one side and then the other of a bluff body. It also states that the frequency of shedding is proportional to the velocity across the body. The vortices are counted and used to develop a signal linearly proportional to the flow rate. Upp and LaNasa state that the "vortices may be counted in many ways since the vortex represents a pressure and temperature change, and either of these may be sensed." 12 According to Perry et al., "accuracy can be maintained regardless of density, viscosity, temperature, or pressure when the Reynolds number is greater than 10,000" (ref. 11). However, there is usually a low-flow cutoff point below which the meter output is zero; the specific point is highly dependent on the geometry of the bluff body. ${ }^{9}$ This flowmeter is recommended for use with relatively clean low-viscosity liquids, gases, and vapors. Vortex-shedding flowmeters are also applicable to high-temperature gas and steam. A sufficient length of straight-run pipe is necessary upstream and downstream to prevent distortion in the fluid velocity profile. ${ }^{11}$ The advantages and disadvantages of the vortex-shedding flowmeter are listed below ${ }^{12}$ :

\section{Advantages}

1. The meter has relatively wide rangeability with linear output.

2. With clean fluids, the meter has a history of long-term stable operation.

3. Frequency output can be read directly into electronic readout systems.

4. Installation costs are moderate.

5. Installation is simple. 
6. When minimum or higher Reynolds numbers pertain, effects of viscosity, pressure, and temperature are minimal.

7. No moving parts are in contact with stream.

\section{Disadvantages}

1. Flow into a meter must be swirl free, which requires a straightening vane and/or long straight piping.

2. Output may have frequency instability and/or fade in certain areas of operation, a factor that would affect readout requirements.

3. The meter is not available in sizes greater than 8 in.

4. The pulse train is irregular.

5. The pulse resolution is the same for all meter sizes.

6. The meter is subject to range limitations at lower Reynolds numbers.

\subsubsection{Turbine Flowmeters}

A turbine flowmeter is actually a velocity-measuring device. ${ }^{12}$ A bladed rotor is suspended axially in the flow stream and spins at a rate proportional to the fluid velocity. ${ }^{10}$ The speed can be measured accurately by counting the rate at which turbine blades pass a given point. According to Perry et al., "turbine meters are available with full-scale flow rates ranging from about 0.1 to $30,000 \mathrm{gal} / \mathrm{min}$ for liquids and 0.1 to $15,000 \mathrm{ft}^{3} / \mathrm{min}$ for air." ${ }^{, 11}$ Nonlinearity can be less than $0.05 \%$ in the larger sizes. Pressure drop across the meter varies with the square of flow rate and is approximately 3-10 psi at full flow. McCabe et al. state that "turbine meters are exceptionally accurate when used under the proper conditions, but they tend to be fragile and their maintenance costs may be high." 10 The advantages and disadvantages of the turbine flowmeter are listed below ${ }^{12}$ :

\section{Advantages}

1. Good accuracy exists over the full linear range of the meter.

2. Electronic output is available directly at a high resolution rate, which makes calibration possible in a short time period with smaller times or volumes.

3. The cost of the meter itself is midrange, but the total meter station is of low to medium cost because of the high flow rate range for a given line size.

4. Although the meter has pressure and temperature limits, it can handle normal flow conditions very well.

5. The meter has shown excellent rangeability on gas meters at high pressure.

\section{Disadvantages}

1. The meter requires throughput calibration to establish the most accurate use.

2. Viscosity affects liquid meters, which may require separate calibration curves for different viscosities.

3. The rangeability at low pressures is about the same as those of other gas meters.

4. The upstream flow pattern must be nonswirling, which necessitates the use of straightening vanes.

\subsubsection{Magnetic Flowmeters}

The operating principle of magnetic flowmeters is Faraday's law of electromagnetic inductance. Perry et al. explain this principle as follows: "The magnitude of the voltage induced in a conductive medium moving at right angles through a magnetic field is directly proportional to the product of the magnetic flux density, the velocity of the medium, and the path length between the probes." 
approach requires that the liquid to be measured have a minimum conductivity; therefore, this meter is not applicable to hydrocarbons. Since induced voltage depends on velocity only, changes in the density or viscosity of the liquid have no effect on the meter reading. ${ }^{10}$ These meters are inherently nonintrusive, because the magnetic field and subsequent voltage measurement can be generated and sensed externally. ${ }^{9}$ Since the meter is full line size, it causes no pressure drop other than normal pipe loss. Magnetic flowmeters are very accurate over wide flow ranges and are especially accurate at low flow rates. According to Perry et al., "the pressure of multiple phases or undissolved solids can affect the accuracy of the measurement if the velocities of the phases are different than that for straight-run pipe." "11 Typical applications of magnetic flowmeters include metering viscous fluids, slurries, or highly corrosive chemicals. Because magnetic meters should be filled with fluid, the preferred installation is in vertical lines with upward flow. The meters are fairly expensive and have a high operating cost because of the high power requirements. The advantages and disadvantages of the magnetic flowmeter are listed below ${ }^{12}$ :

\section{Advantages}

1. The performance is not affected by changes in viscosities and densities.

2. The full-bore opening means no head loss.

3. The meter will operate bidirectionally with required upstream lengths installed on both sides.

4. The meter is available with insert liners, which allow use on some corrosive and erosive fluids.

\section{Disadvantages}

1. Installation and operating costs are relatively high because of size, weight, and electrical power costs.

2. Fluids must have at least the minimum conductivity specified by manufacturer of the specific meter.

3. The meter can be used for liquids and slurries but not for gases.

\subsubsection{Ultrasonic Flowmeters}

All ultrasonic flowmeters are based on an ultrasonic signal being changed by or reflected from the flowing stream velocity. Two fundamental measurement techniques, depending upon liquid cleanliness, are generally used: transit time ("time of flight") and Doppler shift. The meters use wetted or clamp-on-type transducers. According to Hardy et al., the ultrasonic meter "can be nonintrusive, has no moving parts, and induces no pressure loss." ${ }^{9}$ Even though ultrasonic flowmeters are not highly accurate, they can be useful in many types of service, including measuring the flow rate of corrosive fluids. ${ }^{10}$

In the transit-time technique, two opposing transducers are inserted in a pipe so that one transducer is downstream from the other. The transducers are used to measure the difference between the velocity at which the sound travels with the direction of flow and the velocity at which it travels against the direction of flow. The differential velocity is measured by one of two methods: (1) direct time delays using sound wave burst or (2) frequency shifts derived from beat-together, continuous signals. The frequency-measurement technique is usually preferred because of its simplicity and independence of the liquid static velocity. ${ }^{11}$ Transit-time meters require a relatively clean liquid to maintain the uniqueness of the measurement path.

The Doppler shift technique is used on liquids and gases with some type of entrained particles or bubbles that are traveling at the same speed as the main body of flow. ${ }^{12}$ These entrained particles are necessary to reflect the ultrasonic signal. A Doppler technique is applied by transmitting sound waves 
along the flow path and measuring the frequency shift in the returned signal from the entrained particles in the process fluid; this frequency shift is proportional to liquid velocity. ${ }^{11}$

The advantages and disadvantages of the ultrasonic flowmeter are listed below ${ }^{12}$ :

Advantages

1. No added pressure drop occurs, since the meter is the same diameter as adjacent piping.

2. If high-frequency pulse rate of output is used, errors from effects of pulsation and fluctuating flow can be minimized.

3. Installation can be simple and relatively inexpensive.

4. The meter exhibits high rangeability.

5. No moving parts come in contact with flowing fluid.

6. The simple mechanical calibration is easily checked.

\section{Disadvantages}

1. Power is required for operation.

2. For a single path or reflective unit, the flow profile must be fully developed for an average velocity to be determined.

3. The initial cost is high.

\subsubsection{Coriolis Flowmeters}

Coriolis meters are true mass meters that directly measure the mass rate of flow, as opposed to volumetric flow. The meter is linear and requires no adjustments for variations in liquid properties. It also eliminates the need to compensate for changing temperature and pressure conditions. The meter is especially useful for measuring liquids for which the viscosity varies with velocity at given temperatures and pressures.

The measuring principle of the Coriolis flowmeter is based on the controlled generation of Coriolis forces. This means that "as a fluid passes through a tube forced into vibration electromechanically, a Coriolis force is generated, which alters the tube's vibration mode." ${ }^{\prime 2}$ These forces are always present when both translational (straight-line) and rotational (revolving) movements occur simultaneously. The amplitude of the Coriolis forces depends on the moving mass; its velocity in the system; and, therefore, its mass flow. The Coriolis forces produced at the measuring tubes cause a phase shift in the tube oscillation (see Fig. 7). When there is zero flow (Fig. 7, Pt. 1), the two phases are equal. When there is mass flow, the tube oscillation is decelerated at the inlet (Fig. 7, Pt. 2) and accelerated at the outlet (Fig. 7, Pt. 3). As the mass flow rate increases, the phase difference also increases (Fig. 7, Pts. 3A and 3B). The oscillation of the measuring tube is determined using electrodynamic sensors at the inlet and outlet. The measurement principle operates independently of temperature, pressure, viscosity, conductivity, or flow profile. Coriolis meters can use a curved (Utube) or straight-tube design; both types operate the same way.

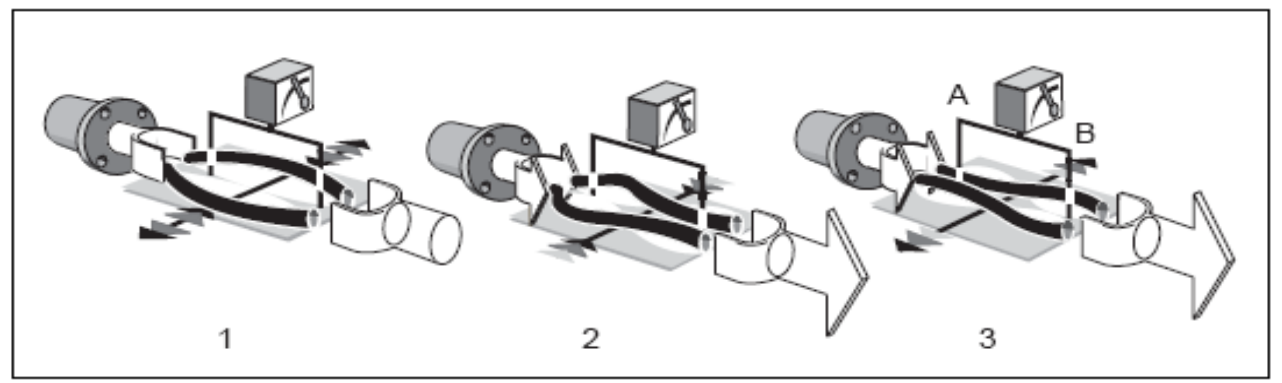

Fig. 7. Coriolis operating principle. The Coriolis forces produced at the measuring tubes cause a phase shift in the tube oscillation. When there is zero flow (i.e., with the fluid standing still) both phases are equal (Pt. 1: no phase difference). When there is mass flow, the tube oscillation is decelerated at the inlet (Pt. 2) and 
accelerated at the outlet (Pt. 3). As the mass flow rate increases, the phase difference also increases (Pts. 3A and 3B). The oscillation of the measuring tube is determined using electrodynamic sensors at the inlet and outlet. (Source: www.endress.com.)

Coriolis meters can be used with virtually any liquid and are extremely insensitive to operating conditions. These meters are generally more expensive than volumetric meters and can be costly to install and operate. Due to the circuitous path of flow through the meter, Coriolis flowmeters exhibit higher-than-average pressure changes. ${ }^{11}$ The meter should be installed so that it will remain full of fluid, with the best installation in a vertical pipe with upward flow. According to Perry et al., "there is no Reynolds number limitation with this meter, and it is quite insensitive to velocity profile distortions and swirl." "Consequently, no straight piping is required upstream. Coriolis meters are highly accurate and can be used on liquids and most gases. Most models offer both mass rate and density measurement via one device (although the meters do not measure gas density accurately). ${ }^{12}$ The advantages and disadvantages of the Coriolis flowmeter are listed below ${ }^{12}$ :

\section{Advantages}

1. The meter can be used on liquids, slurries, gases, and two-phase liquids and gas flows (within set limits).

2. Mass is measured directly.

3. The meter can handle difficult fluids in environments in which other meters cannot be used.

4. The meter provides high accuracy and repeatability on liquid flow and density. The accuracy is comparable to that of other meters generally used on liquid flow.

5. The turndown ratio for the meter is high.

6. Operation is independent of swirl and flow profile, and no flow conditioning is required.

7. Pressure ratings, low-flow limits, and noise immunity have been greatly improved in recent years by some manufacturers.

\section{Disadvantages}

1. The meter is available only in sizes of $1 / 16$ to 6 in.

2. Volumetric flow rates are not measured directly.

3. Special installation requirements are necessary to isolate some meters from mechanical vibration.

\subsection{FLOWMETER SELECTION}

Approximately 50 instruments were investigated; see Appendix A for a listing of these instruments and their specifications. The following three were selected for testing in the flow loop at Oak Ridge National Laboratory (ORNL): the Endress+Hauser Promass 83F Coriolis flowmeter, the Yokogawa ADMAG SE magnetic flowmeter, and the Controlotron 1010N1 transit-time ultrasonic flowmeter (see Fig. 8). The search for commercially available instruments to measure the uranium concentration in a liquid stream was not successful. 

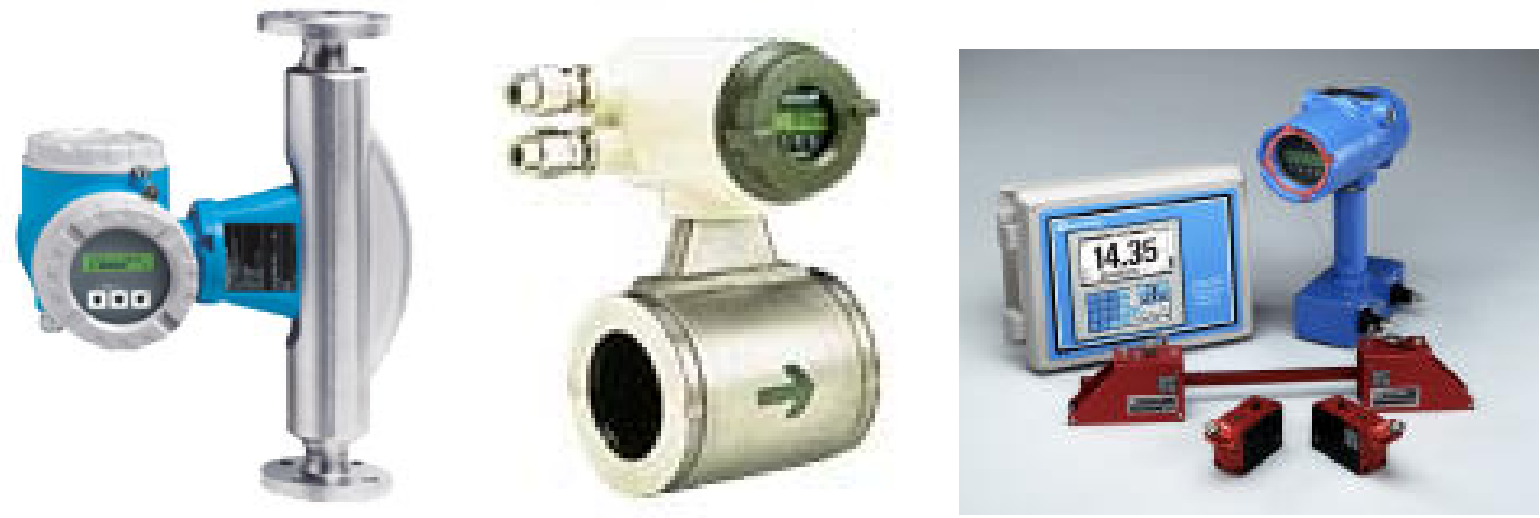

Fig. 8. Flowmeters selected for installation in the ORNL uranyl nitrate flow loop. The instruments (left to right) are as follows: Endress+Hauser Coriolis meter, Yokogawa magnetic flowmeter, and Controlotron ultrasonic meter.

The Endress +Hauser Promass 83F Coriolis meter was selected because of its ability to measure density as well as mass and volumetric flow rates with excellent accuracy and repeatability. This meter can also measure temperature. For the size purchased ( $3 / 8 \mathrm{in}$.), the mass flow rate measurement range was $0-2000 \mathrm{~kg} / \mathrm{h}$, with a reported accuracy of $\pm 0.1-0.15 \%$ and a repeatability of $\pm 0.05 \%$. The accuracy and repeatability of the measurement make the meter suitable for a wide range of applications. The Coriolis flowmeter has a dual-tube sensor with a fully welded design. Process fittings, welded directly to the flow splitter, eliminate the need for internal gaskets, reducing the risk of leakage and ensuring process safety. More information is available at www.endess.com.

The Yokogawa ADMAG SE magnetic flowmeter was selected because of its level of accuracy and ability to measure low flow rates. The flow range was $0.3-10 \mathrm{~m} / \mathrm{s}$, with a reported accuracy of $\pm 0.5 \%$ and a repeatability of $\pm 0.1 \%$. More information is available at www.yokogawa.com/us.

The Controlotron 1010N1 clamp-on transit-time ultrasonic meter was selected because of its nonintrusive nature. The flow range was $0-12 \mathrm{~m} / \mathrm{s}$, with a reported accuracy of $\pm 0.5-1 \%$ and a repeatability of $\pm 0.15 \%$. More information is available at www.controlotron.com.

\section{OPERATION}

Before any testing could take place, the instruments had to be set up. Each instrument was factory calibrated (where this option was available). The Coriolis meter required that the desired output be specified for the output channels. In this case, the desired outputs were total mass flow rate and density, using 4-20-mA current outputs. The magnetic flowmeter had only one output: volumetric flow rate using 4-20-mA current output. The ultrasonic flowmeter was much more difficult to program. Information about pipe diameter and wall thickness, fluid density and viscosity, and temperature range was all required. Because the ultrasonic was a clamp-on-type meter, it was necessary to properly place the transducers in the system. The ultrasonic meter also required the sonic velocity to be determined using the flowmeter computer to transmit a signal through the fluid when the pipe was full but no fluid was flowing.

During each test, data were recorded from each of the instruments [mass flow rate (in kilograms per minute) and density (in grams per cubic centimeter) from the Coriolis meter, volumetric flow rate (liters per minute) from the magnetic meter, and volumetric flow rate (liters per minute) from the ultrasonic meter]. Use of the ultrasonic meter was later discontinued due to meter programming 
issues. Temperature (in degrees Celsius) and pressure (in pounds per square inch) were also recorded during each test.

\subsection{CALIBRATION}

The system was calibrated using two different liquid flow streams: water and uranyl nitrate. Water was used because it could safely be pumped out of the system through the sample port to get a physical measurement of total solution pumped over a specified time period. The calibration with water was performed by flowing water out of the sample port and collecting the water over a period of time; the flow rate was then determined by obtaining the mass of water collected over that time period. The water calibration was repeated by flowing water through the system and collecting data from the instruments. This second procedure was necessary to check the calibration of the instruments with the data that were obtained when the water was pumped out of the sample port, which is located upstream of the instruments. Once uranyl nitrate solution was added to the system, it was not feasible to use the sample port for calibration. The uranyl nitrate calibration was done only using the instruments and did not use the sample port. Uranyl nitrate solution was circulated in the loop, and data were recorded using the instruments. This information was then compared with the water calibration.

\subsection{FLOW RESPONSE}

Several flow response tests were performed. The first was a continuous steady-state flow response test. During this test, the loop was allowed to operate at a specified flow rate for $8 \mathrm{~h}$ with no disturbances. This test simulated stable continuous operation in a plant and was performed only for low pump speeds $(3.6-11.9 \mathrm{~Hz})$. The low pump speeds correspond to linear velocities that would be expected at an NUCP. A total of eight tests were performed for $8 \mathrm{~h}$ over pump speeds of 3.6-11.9 Hz.

The second test was performed to assess the response of the instruments over the range of flow rates available with this system $(\sim 0-11 \mathrm{~L} / \mathrm{min})$. During this test, the pump speed was set to a specified value and the system was allowed to run for approximately 30-60 min before the pump speed was increased for the next test.

Both of these test series were repeated for uranyl nitrate solution with entrained organic with $n$-dodecane used to simulate organic contamination. This type of contamination would not be expected in actual operation, except in very small concentrations, unless there was a failure in the solvent extraction process.

\subsection{ENTRAINED AIR RESPONSE}

The entrained air response tests were performed using compressed air injected into the system through an air input line (see Fig. 4). The air flow rate was controlled using a mass flow controller. The inlet flow rate of air was approximately $500 \mathrm{sccm}$, and the test was performed at low pump speeds $(3.6-11.9 \mathrm{~Hz})$. Depending on the type of process used, an actual plant typically allows the solution to settle for a period of hours to days before the process is continued. Therefore, air is not likely to be present in the system. However, this test simulated the possibility of air entrainment in the solution if the solution was not allowed to separate before further processing. A total of eight tests were performed over pump speeds of 3.6-11.9 Hz; each test lasted $15 \mathrm{~min}$. This test series was repeated for uranyl nitrate solution with entrained organic. 


\subsection{PULSATING FLOW RESPONSE}

Pulsating flow response tests were performed by manually cycling the pump on and off. While the pump was operated at low speeds $(3.6-11.9 \mathrm{~Hz})$. Although this is not a typical method of operation in a plant, it provided some information about what might occur during start-up or shutdown. This test also simulated the type of operation of a pulse column that might be used in an NUCP. A total of eight tests were performed over pump speeds of 3.6-11.9 Hz; each test lasted $10 \mathrm{~min}$. This test series was repeated for uranyl nitrate solution with entrained organic.

\subsection{PULSATING FLOW WITH ENTRAINED AIR RESPONSE}

The entrained air and pulsating flow tests were combined to determine how the instruments might react to extremes in the process operation. This test was performed at low pump speeds $(3.6-11.9 \mathrm{~Hz})$ and was repeated for uranyl nitrate solution with entrained organic. A total of eight tests were performed over pump speeds of 3.6-11.9 Hz; each test lasted $10 \mathrm{~min}$.

\section{RESULTS}

\subsection{CALIBRATION}

The system was initially calibrated with water. Figures 9-11 show, respectively, the calibrations of the magnetic meter, the Coriolis meter, and the ultrasonic meter with water. The solid line represents the flow rate of water that was pumped out of the sample port and collected. The calibration of the instruments was completed twice. As shown in Figs. 9 and 10, the magnetic meter and the Coriolis meter were both within calibration. However, the ultrasonic meter was difficult to set up and difficult to operate properly in this application because the material properties of the tubing were not known well enough and the fluid properties varied. As a result this calibration (Fig. 11) was not good and the ultrasonic meter was not used for further testing. 


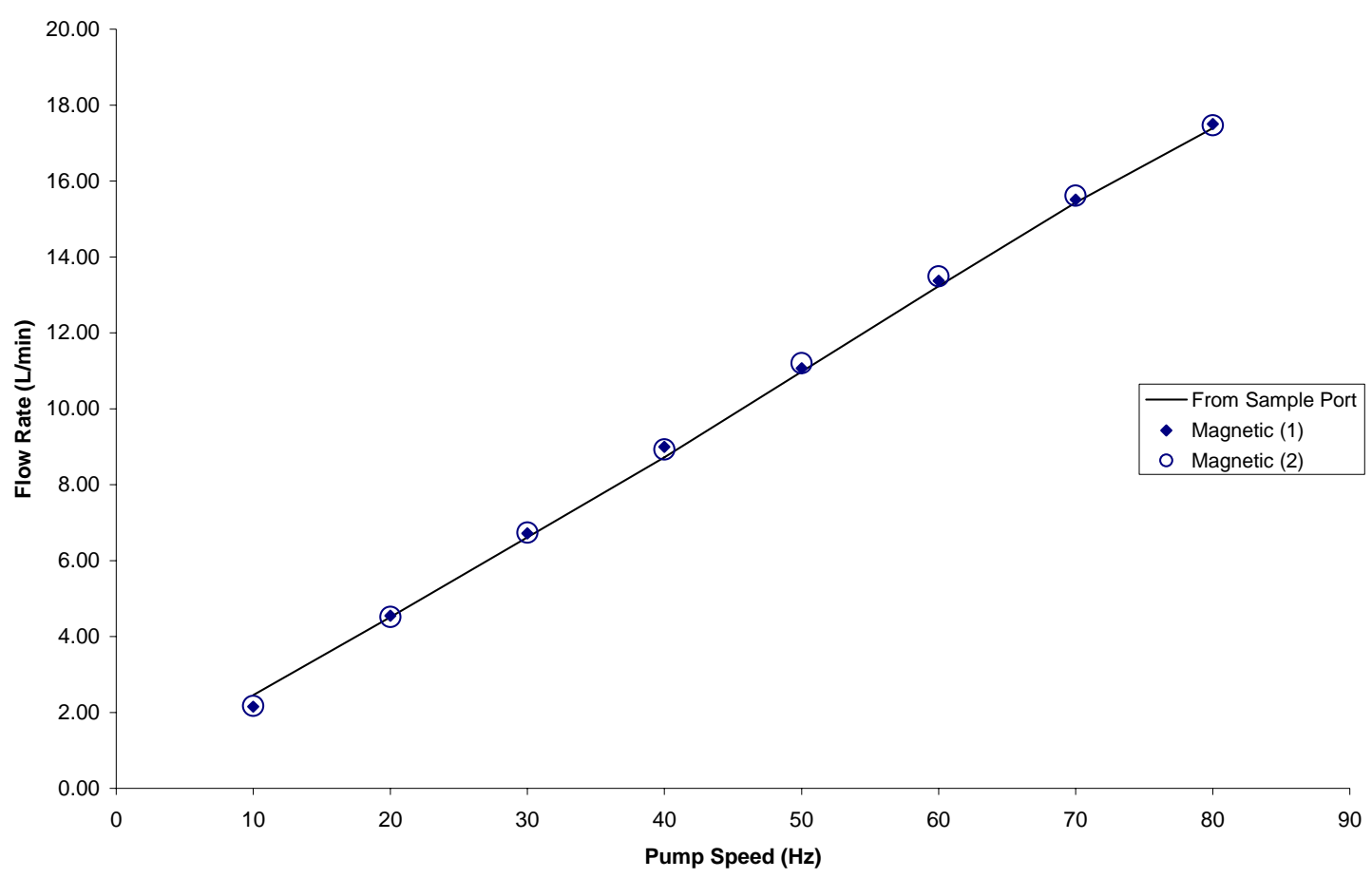

Fig. 9. Magnetic flowmeter calibration with water.

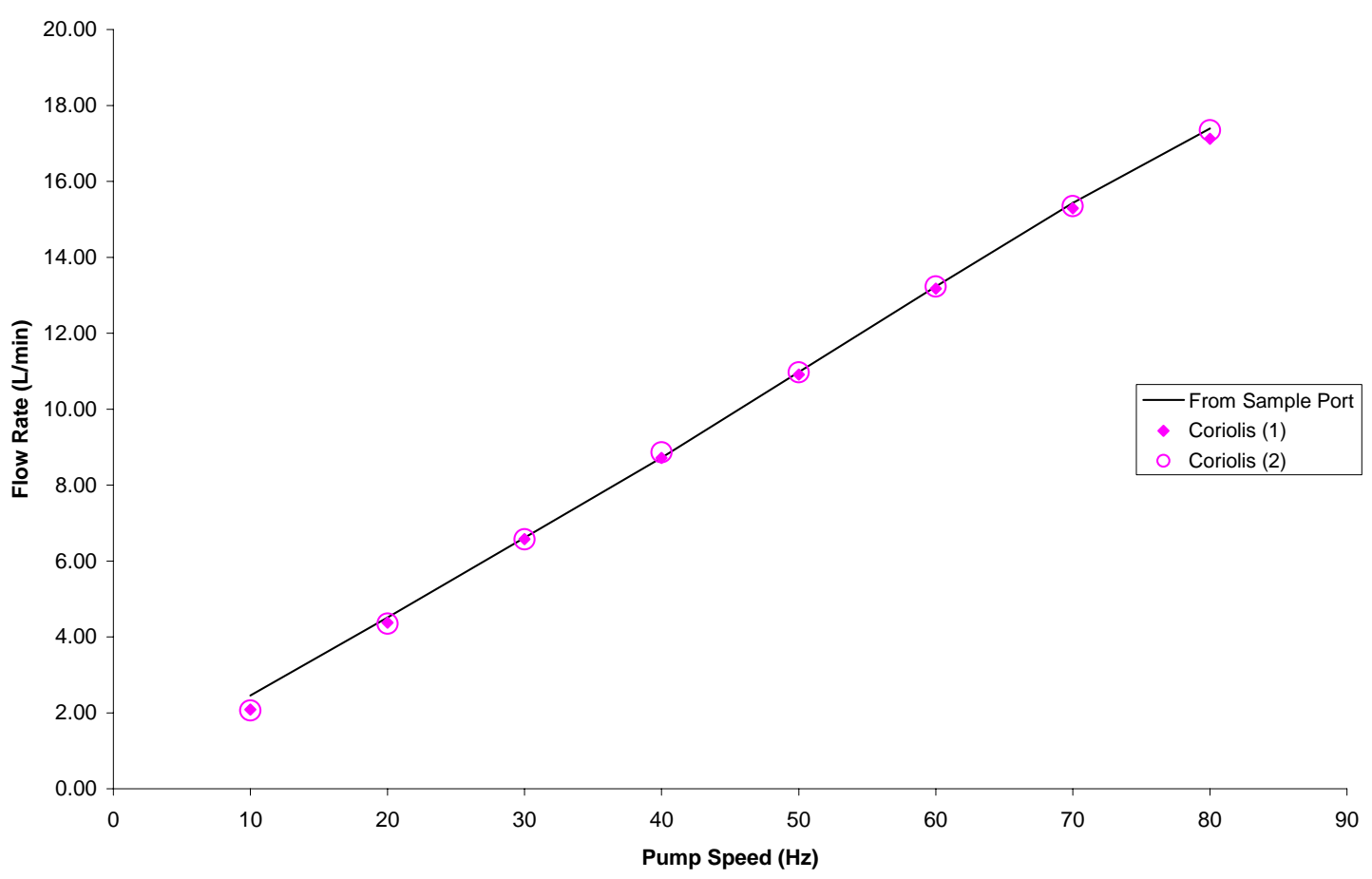

Fig. 10. Coriolis flowmeter calibration with water. 


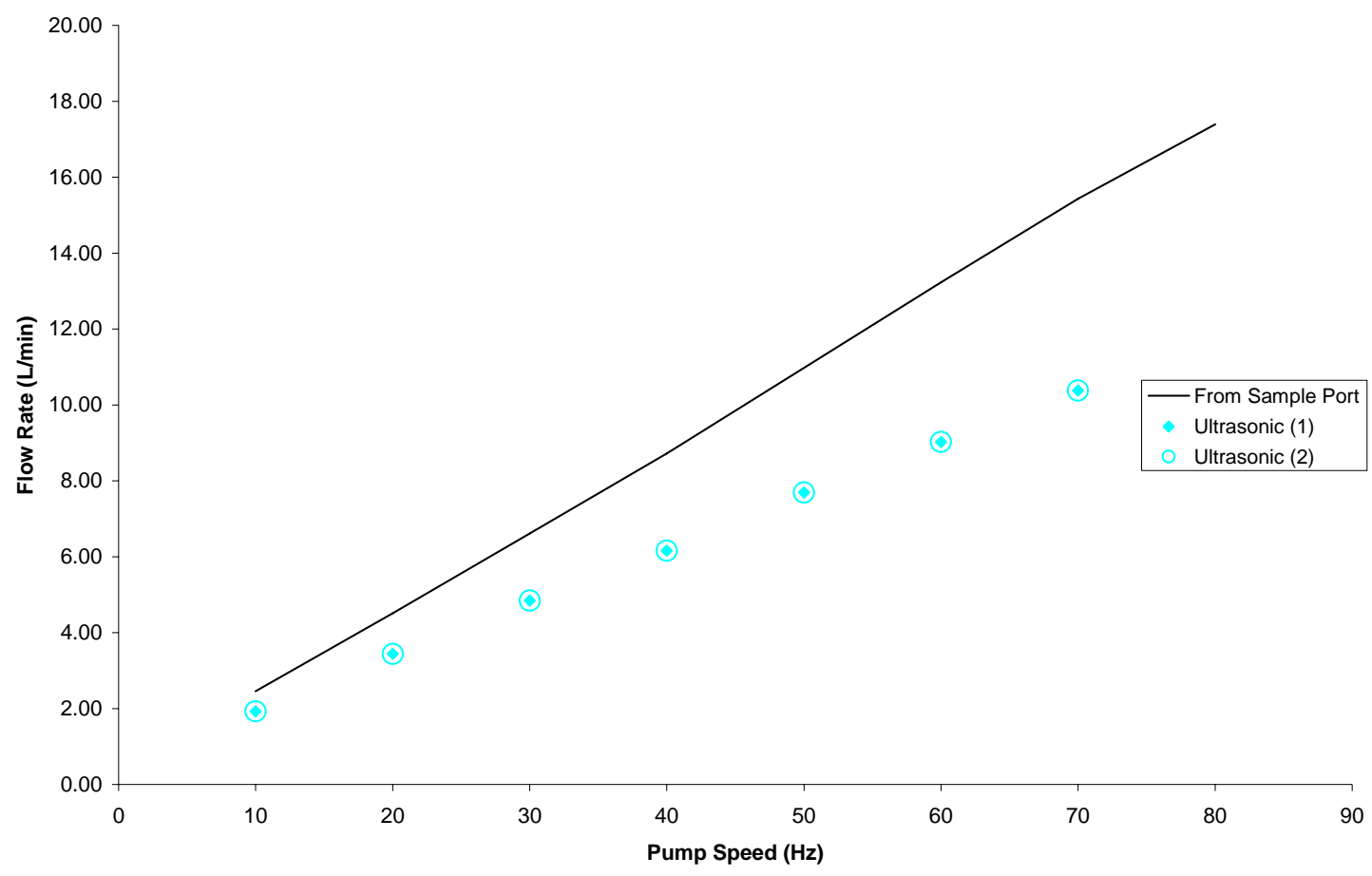

Fig. 11. Ultrasonic flowmeter calibration with water.

Following the calibration with water, uranyl nitrate solution was added to the system and it was no longer possible to use the sample port for calibration purposes. For the calibration with uranyl nitrate, the solution was circulated through the system while data was collected from the instruments and then compared to the calibration with water. Figures 12 and 13 show, respectively, the calibrations of the magnetic meter and the Coriolis meter with uranyl nitrate. Again, both meters were within calibration. Because of the use of a positive displacement pump, the flow rate was expected to remain unchanged. 


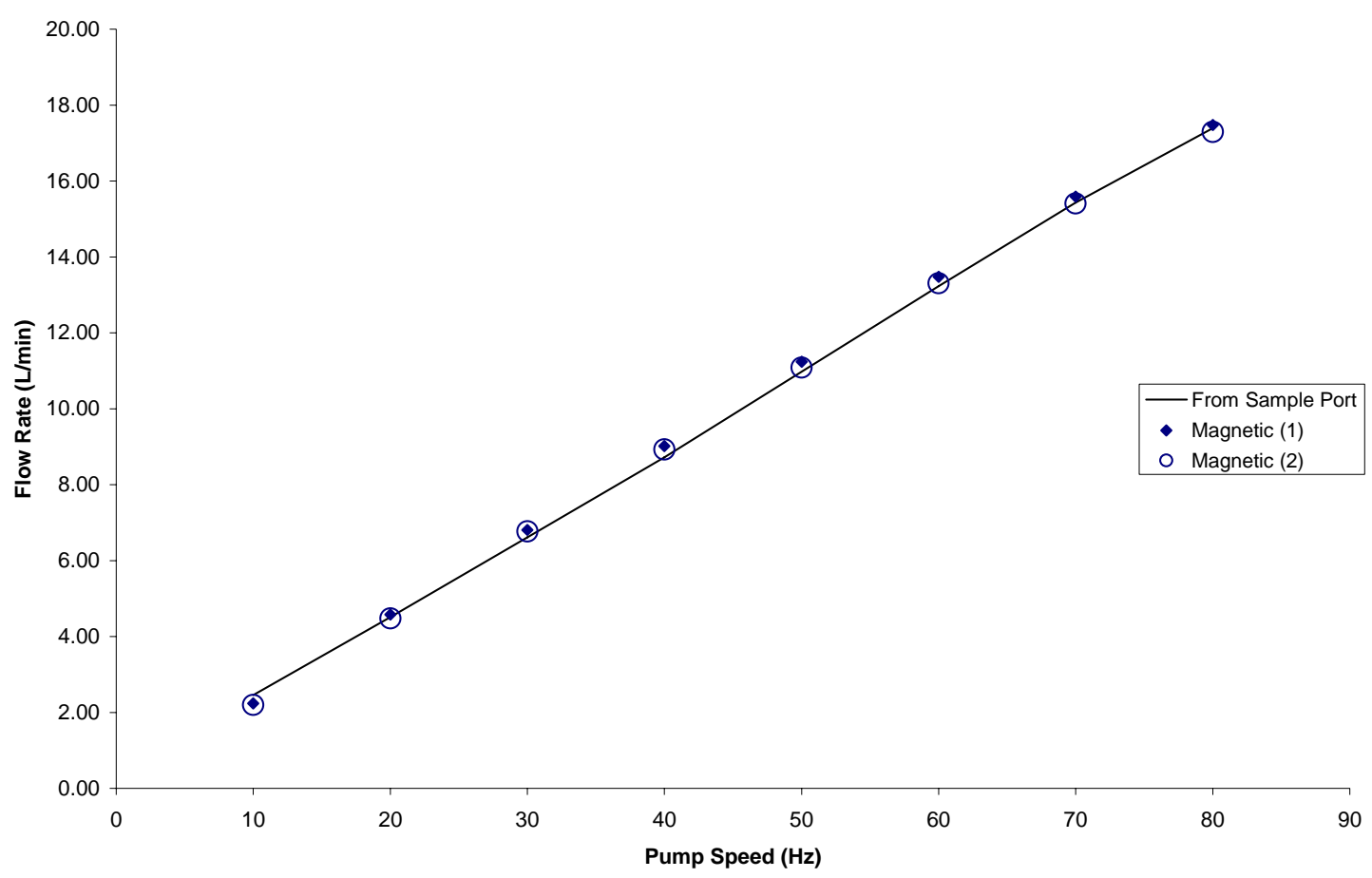

Fig. 12. Initial magnetic flowmeter calibration with uranyl nitrate.

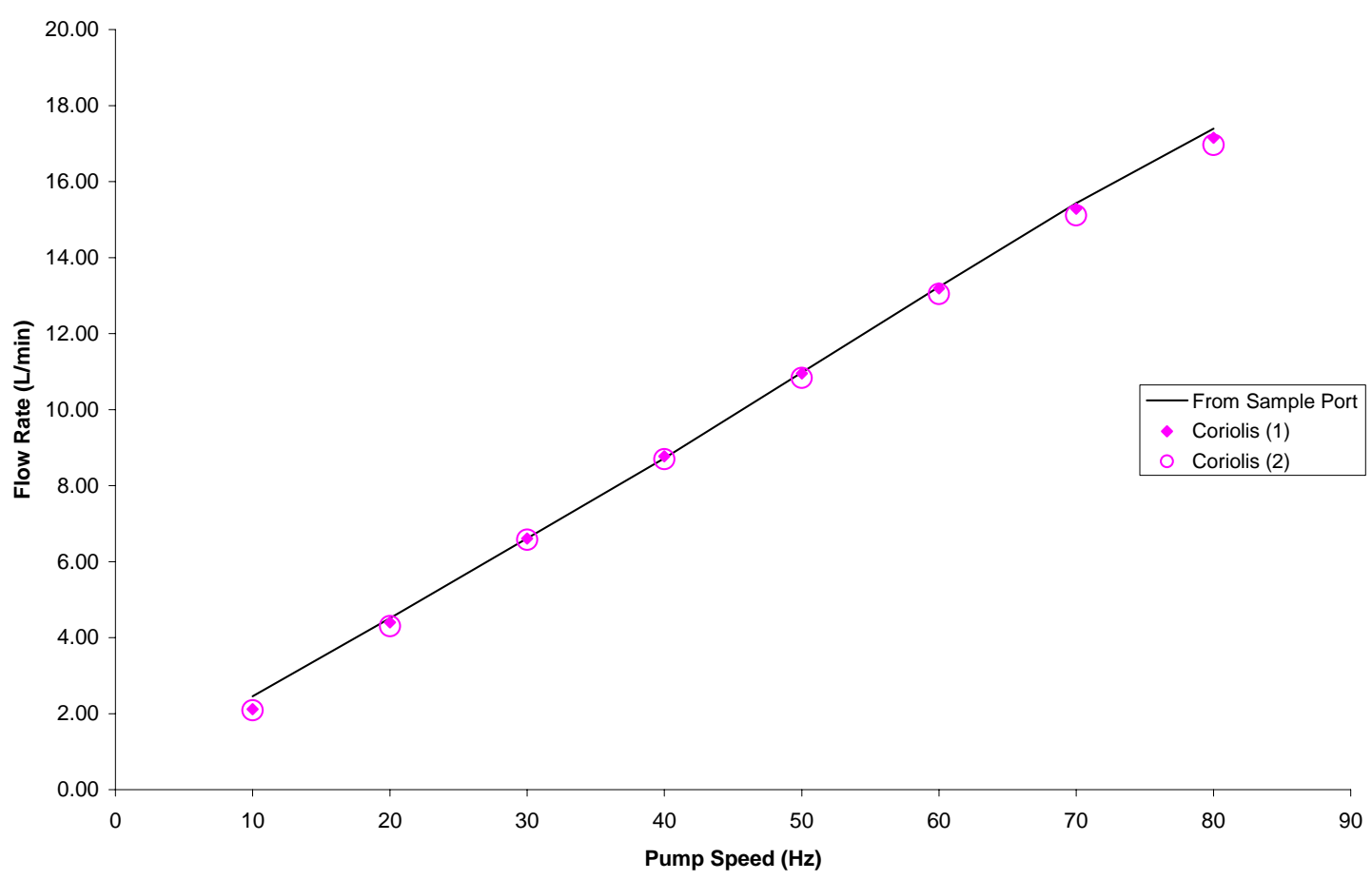

Fig. 13. Initial Coriolis flowmeter calibration with uranyl nitrate. 
In August 2005, degradation of the gear pump resulted in decreased flow rates, as shown in Figs. 14 and 15. The pump head was replaced, requiring recalibration of the system, as shown in Figs. 16 and 17. However, use of the new pump head made comparison with the initial water calibration impossible. Therefore, after the new pump head installation, the calibration was checked periodically against an "initial" calibration with the new pump head. In this new "initial" calibration, uranyl nitrate solution was circulated through the system while data was collected from the instruments. This run was used as a comparison for the future runs. The decreased flow rates (Figs. 16 and 17) show that some degradation of the pump continued.

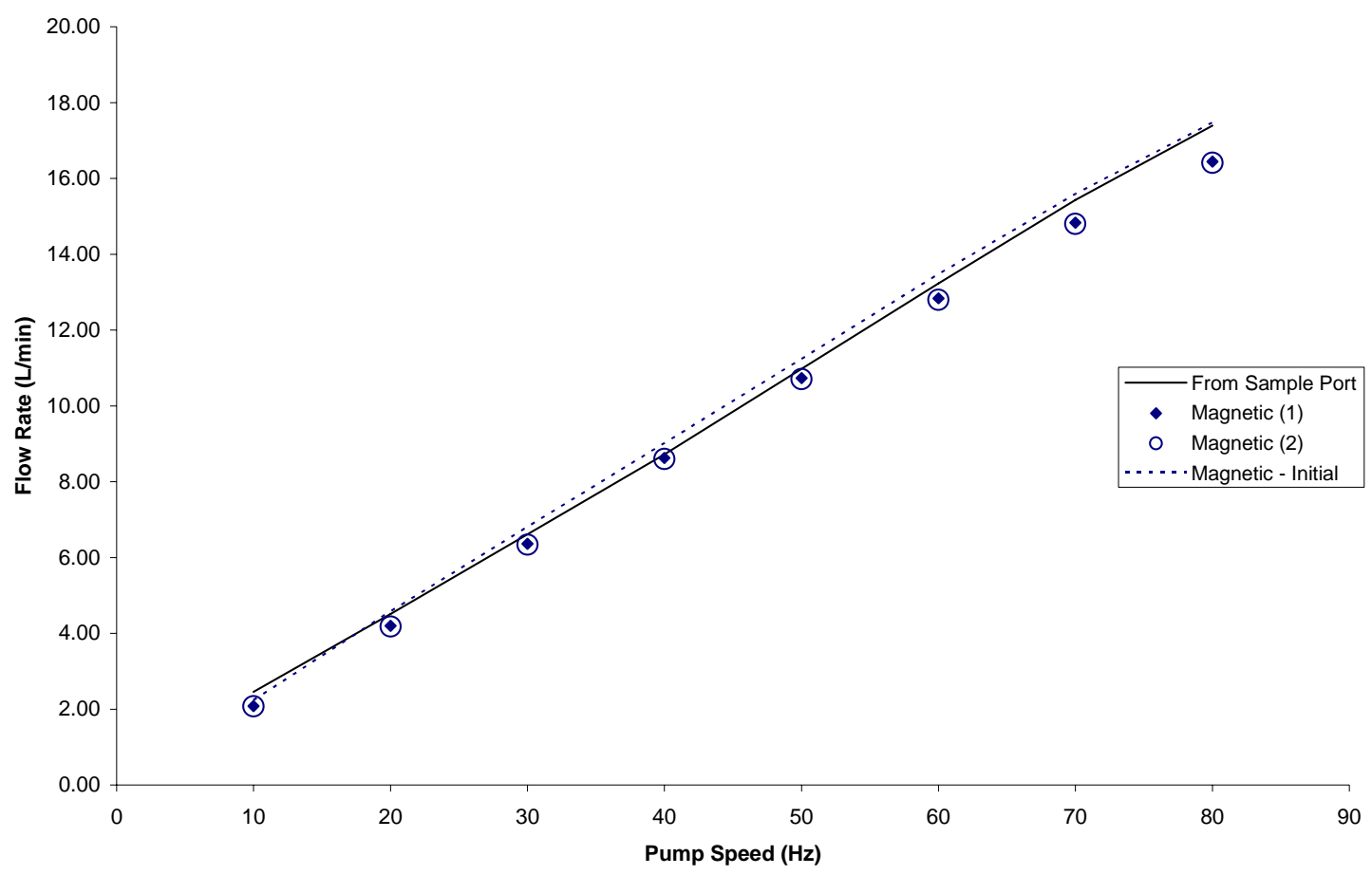

Fig. 14. Magnetic flowmeter calibration with uranyl nitrate following pump failure in August 2005. 


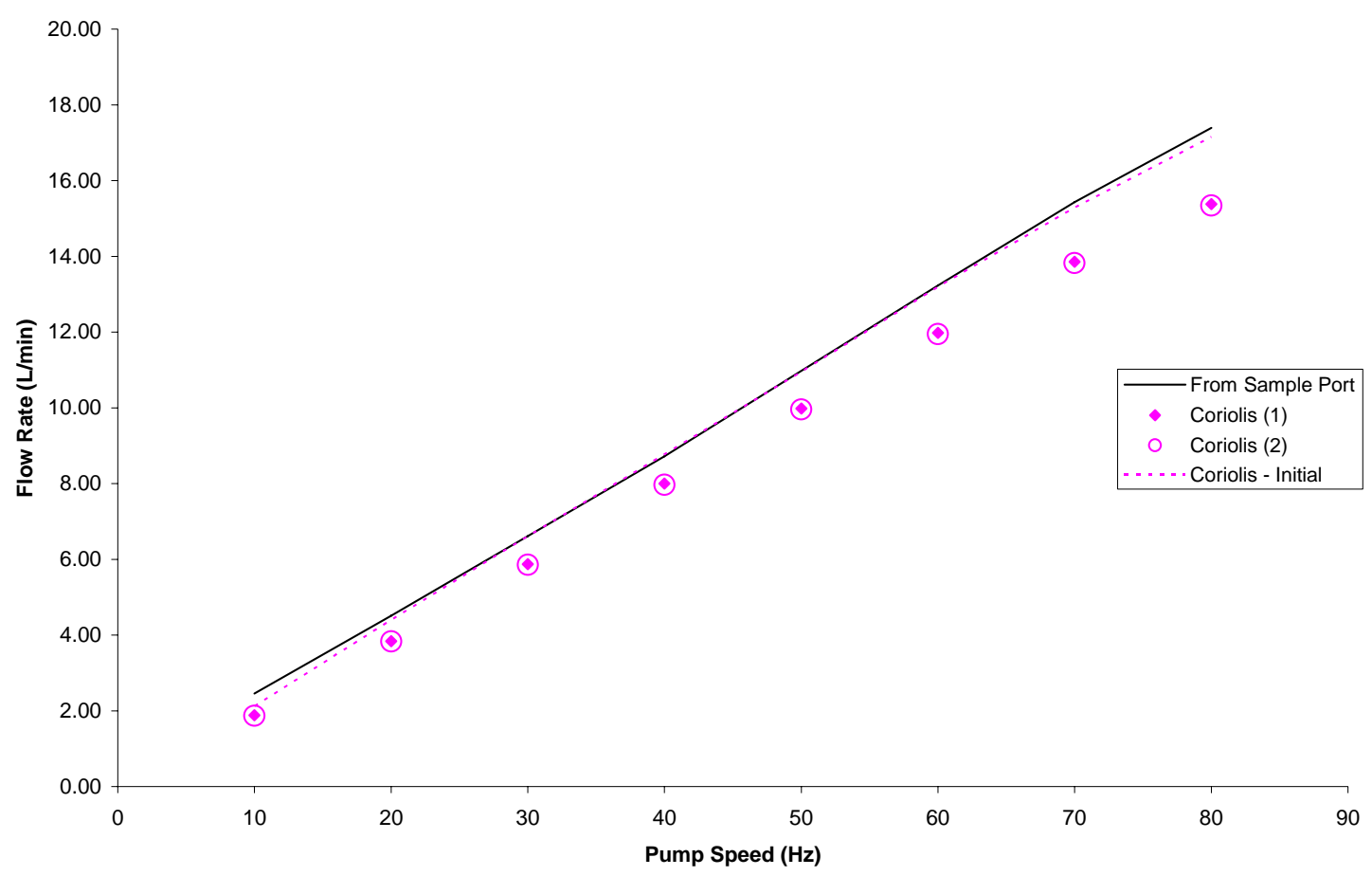

Fig. 15. Coriolis flowmeter calibration with uranyl nitrate following pump failure in August 2005.

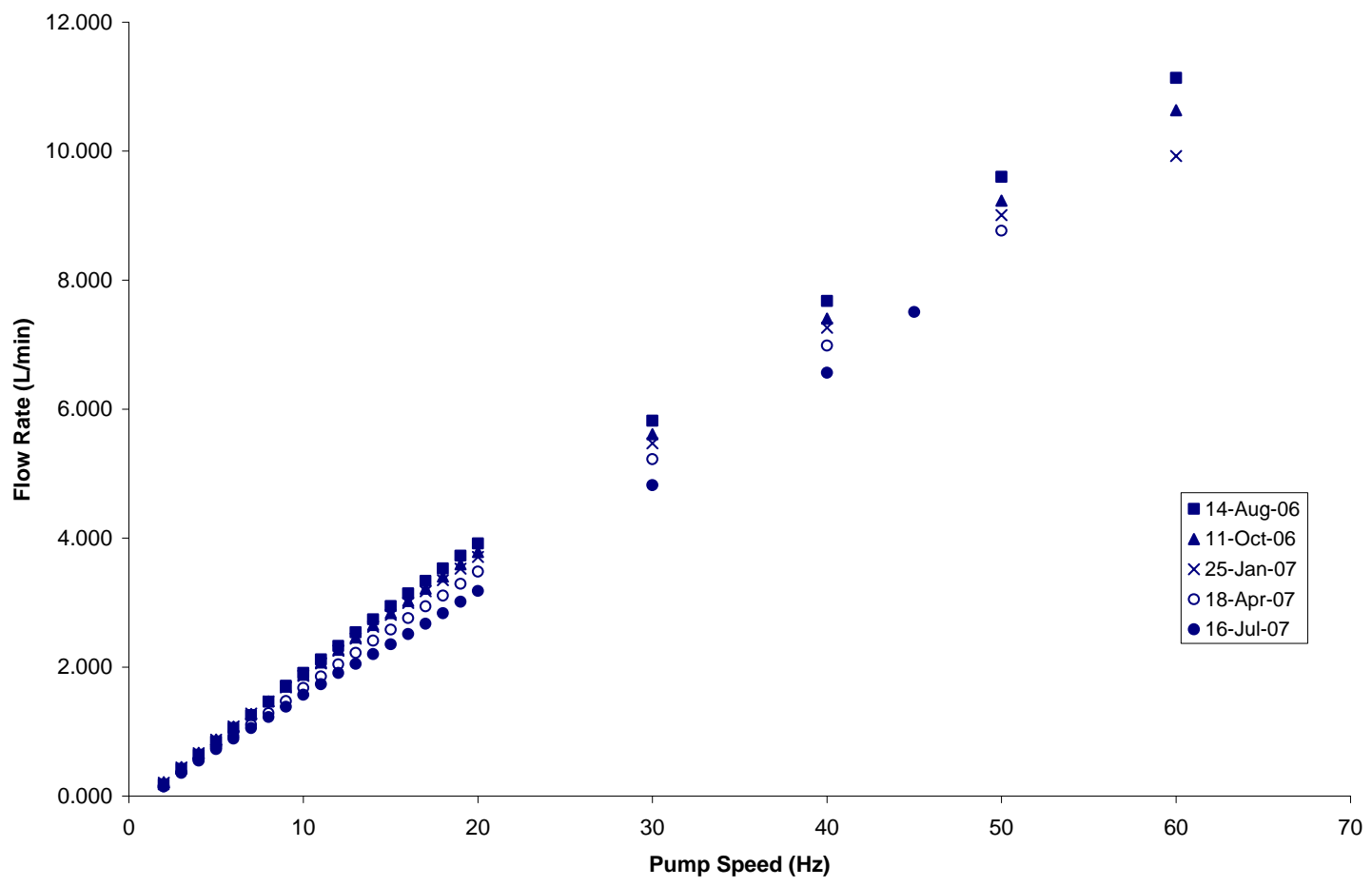

Fig. 16. Magnetic flowmeter calibration with uranyl nitrate following installation of new pump head. 


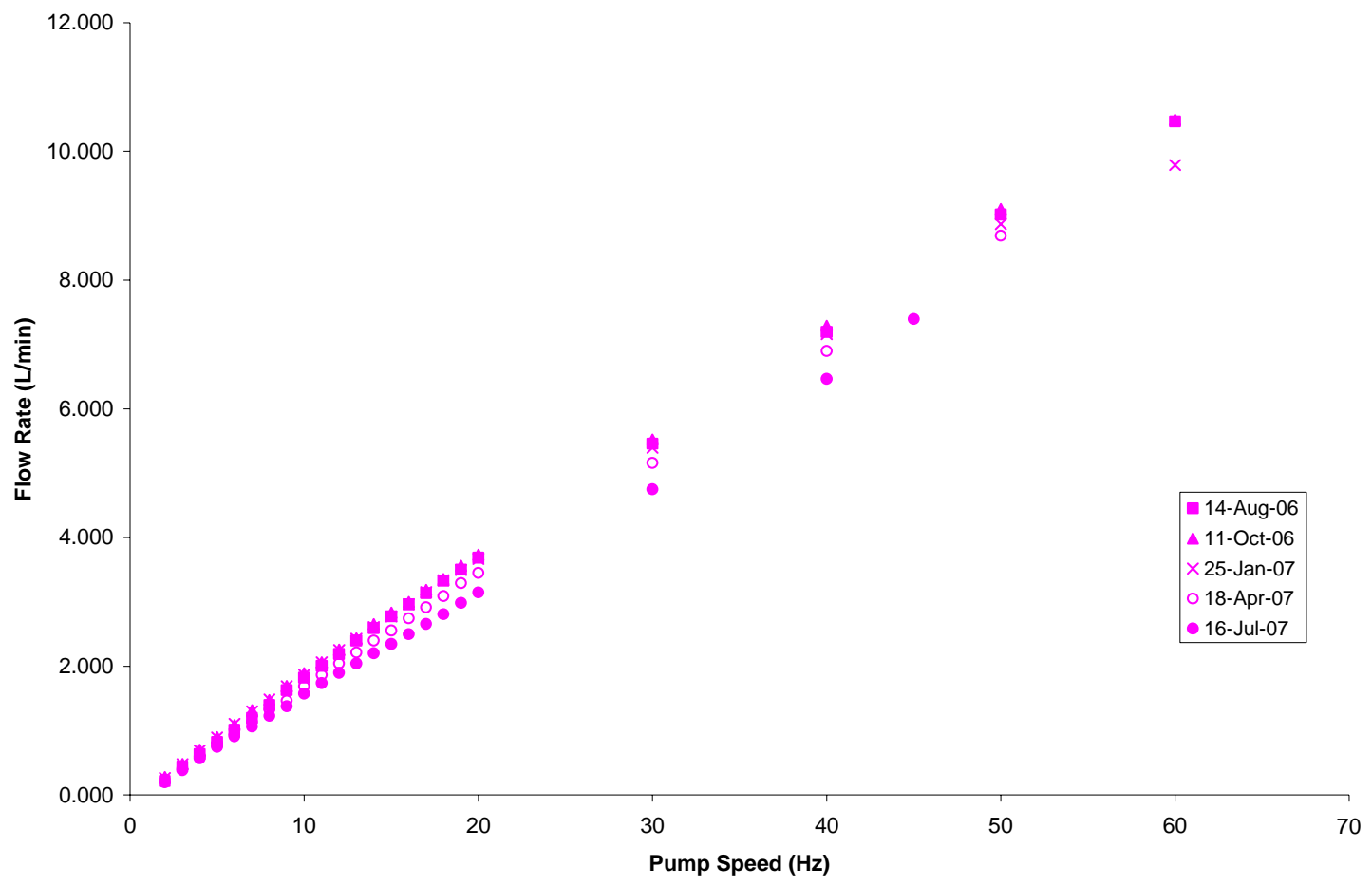

Fig. 17. Coriolis flowmeter calibration with uranyl nitrate following installation of new pump head.

The air entrainment response testing was performed in July 2007. The calibration of the instruments was checked following these tests. Figures 18 and 19 show, respectively, the calibrations of the magnetic meter and the Coriolis meter with uranyl nitrate after the air entrainment tests. 


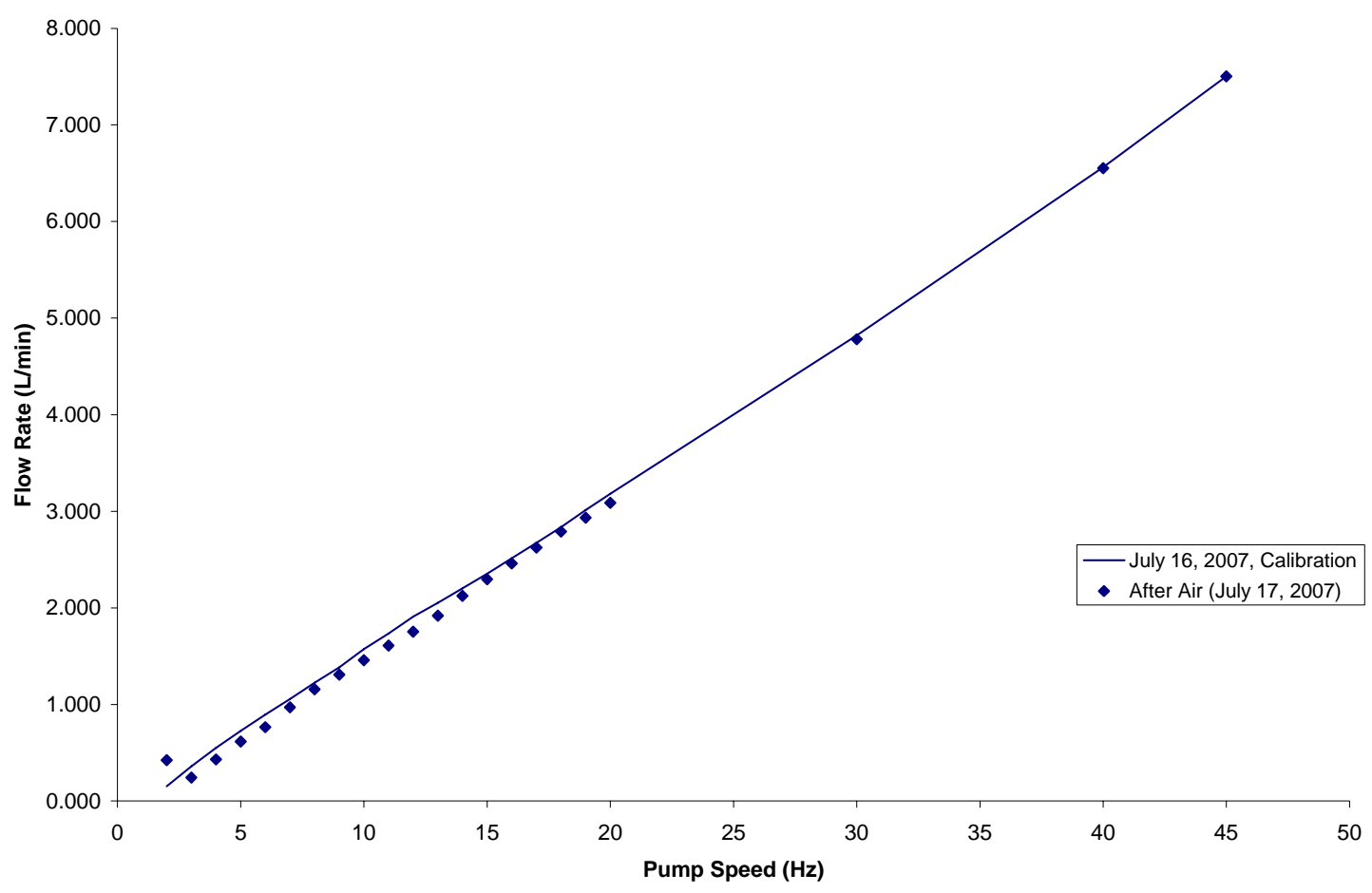

Fig. 18. Magnetic flowmeter calibration with uranyl nitrate following air entrainment testing.

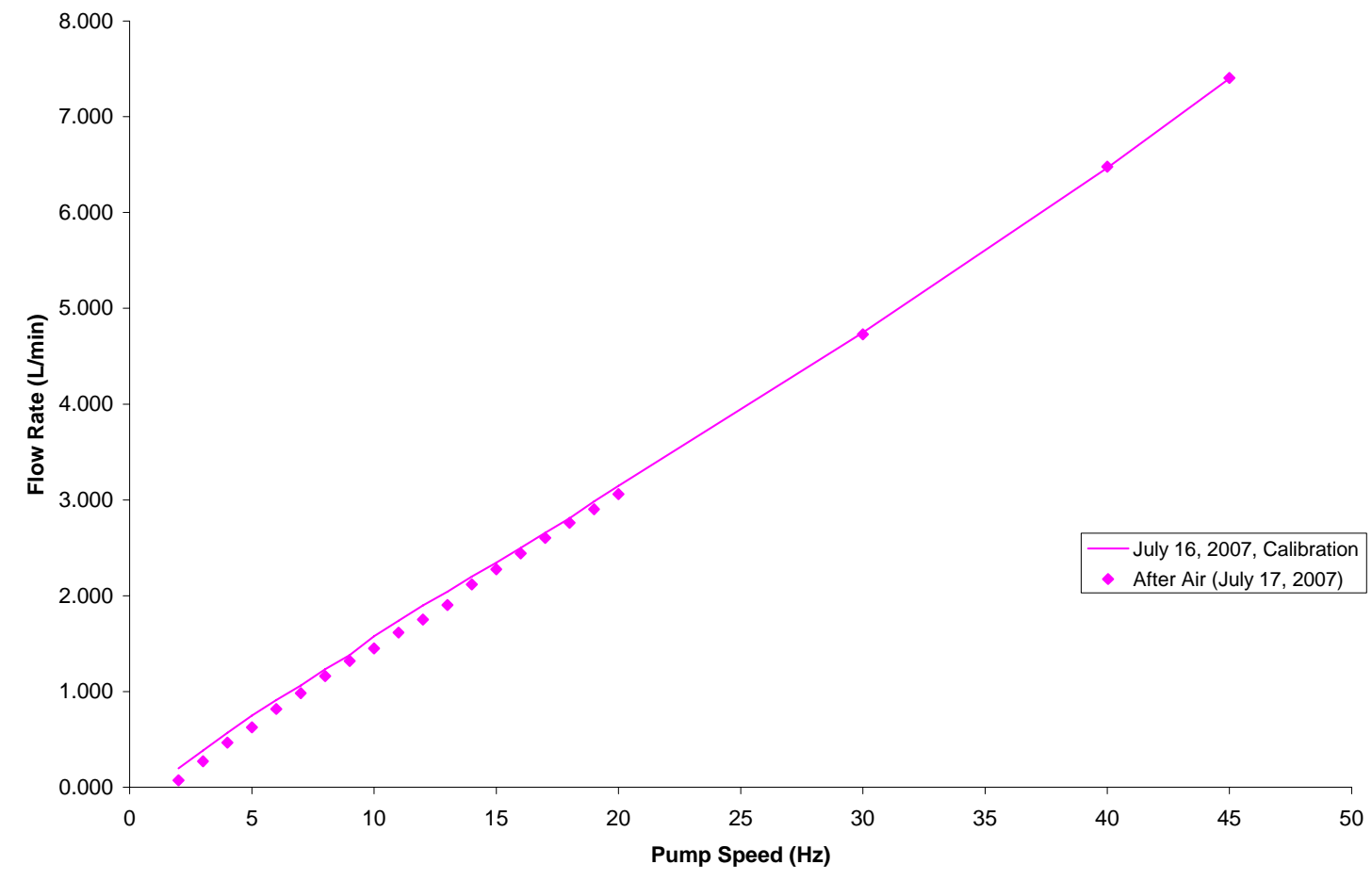

Fig. 19. Coriolis flowmeter calibration with uranyl nitrate following air entrainment testing. 
The entrained organic response testing was performed in July 2007. The calibration of the instruments was checked after the addition of organic contamination into the system. Figures 20 and 21 show, respectively, the calibrations of the magnetic meter and the Coriolis meter with uranyl nitrate subject to organic contamination. Another pump failure occurred before the organiccontamination testing was completed. Figures 22 and 23 show, respectively, the final calibrations of the magnetic and Coriolis meters in comparison with the calibration prior to the addition of organic contamination.

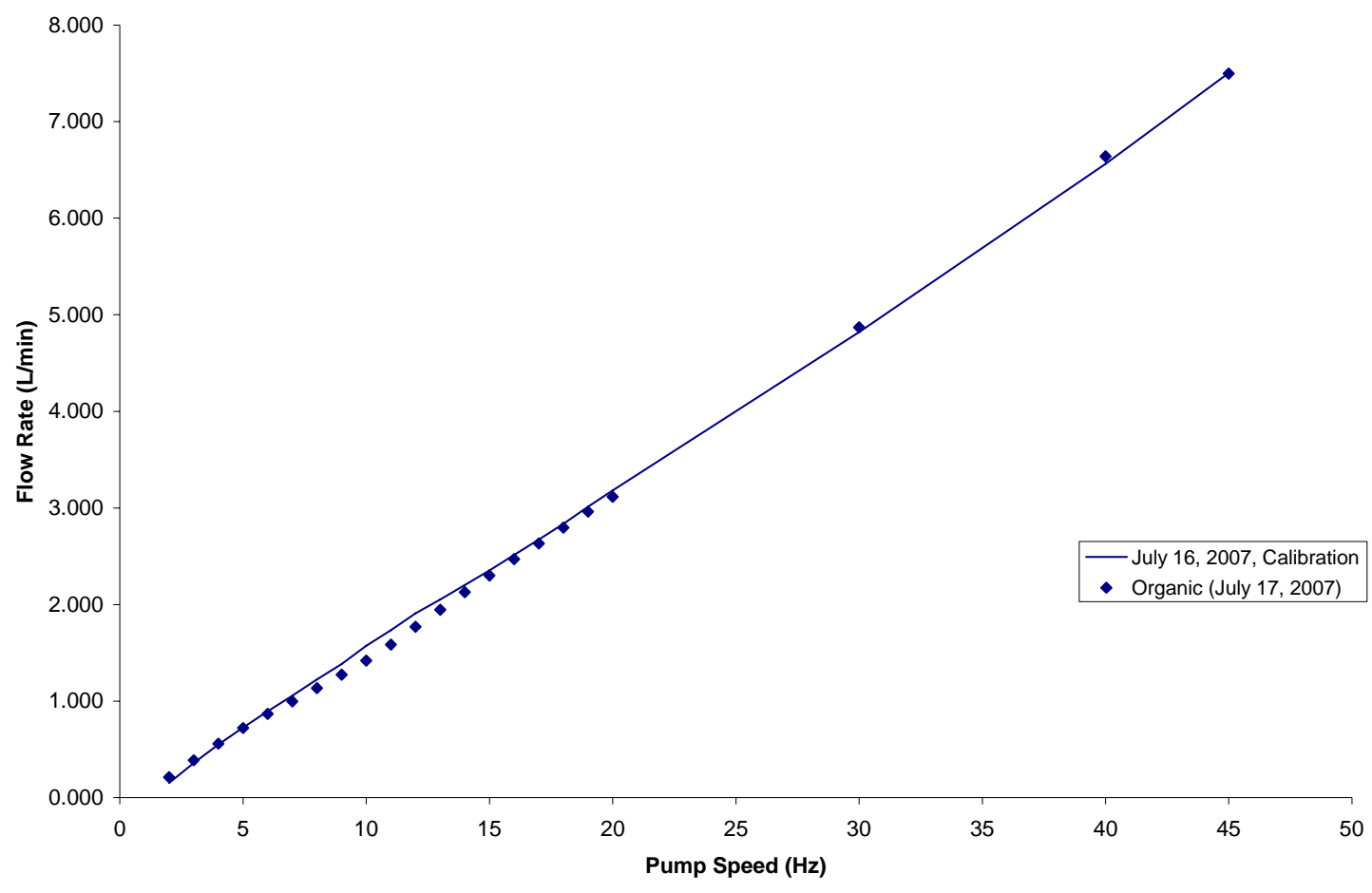

Fig. 20. Magnetic flowmeter calibration with uranyl nitrate and organic contamination. 


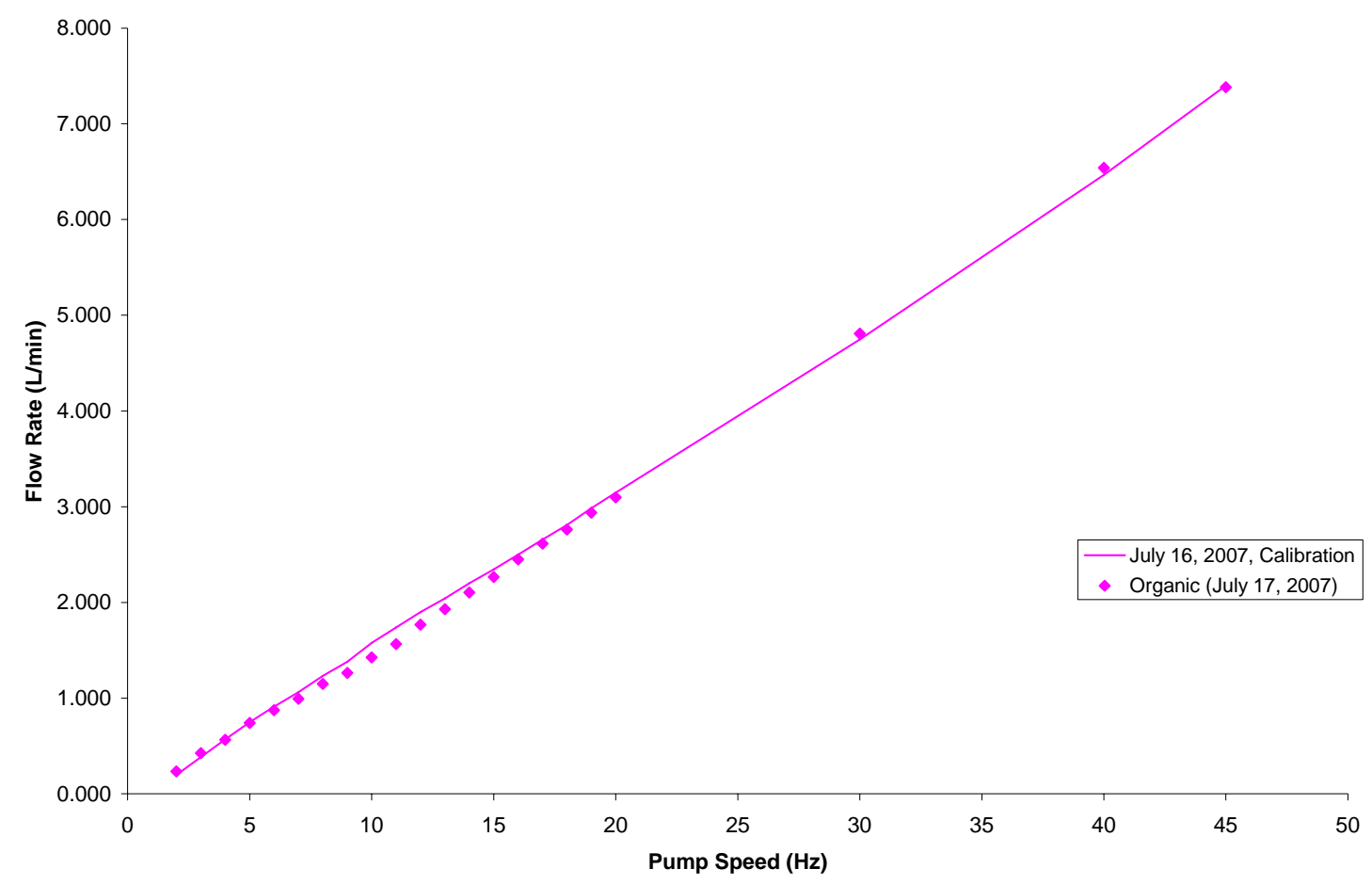

Fig. 21. Coriolis flowmeter calibration with uranyl nitrate and organic contamination.

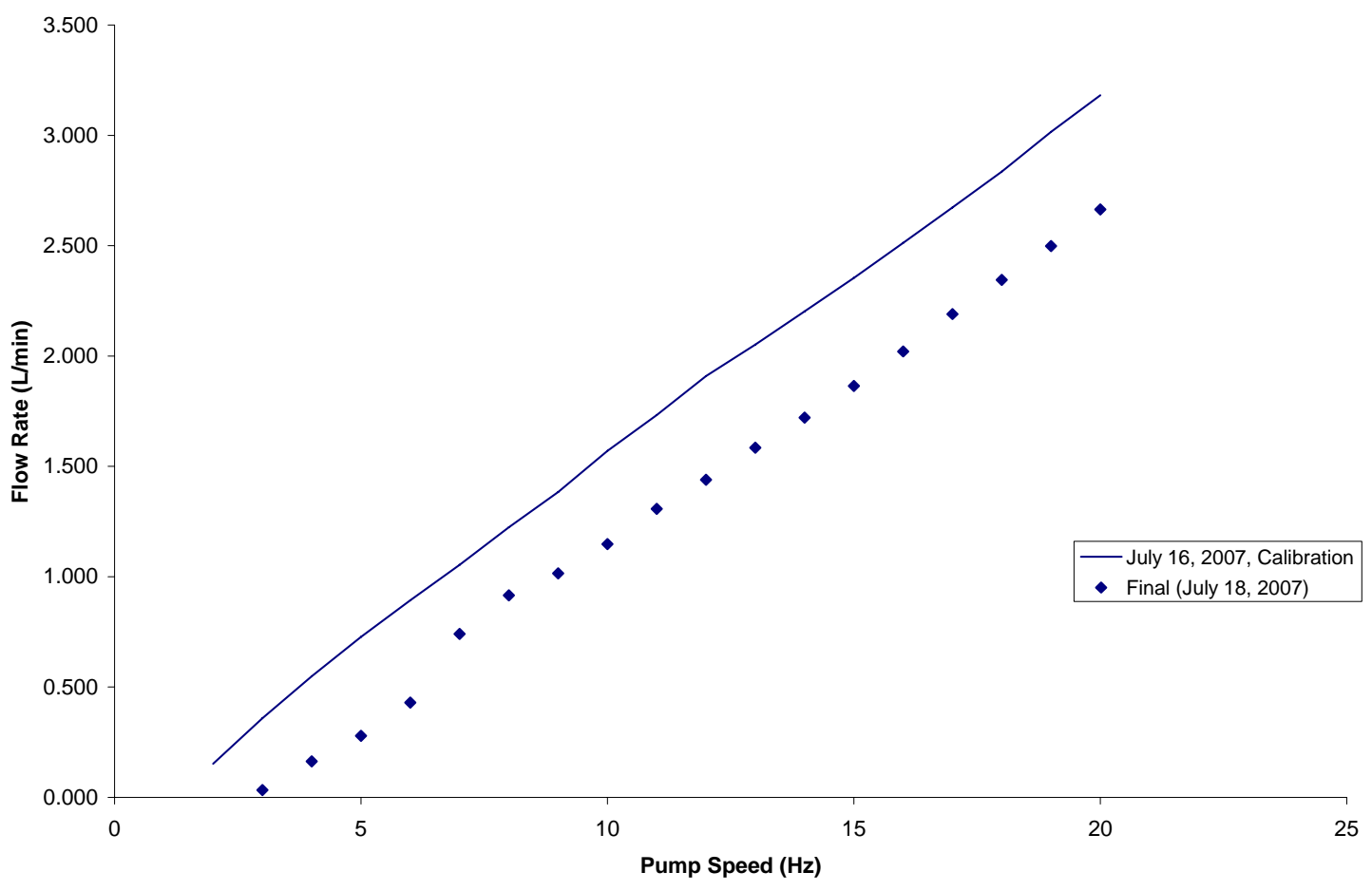

Fig. 22. Final magnetic flowmeter calibration. 


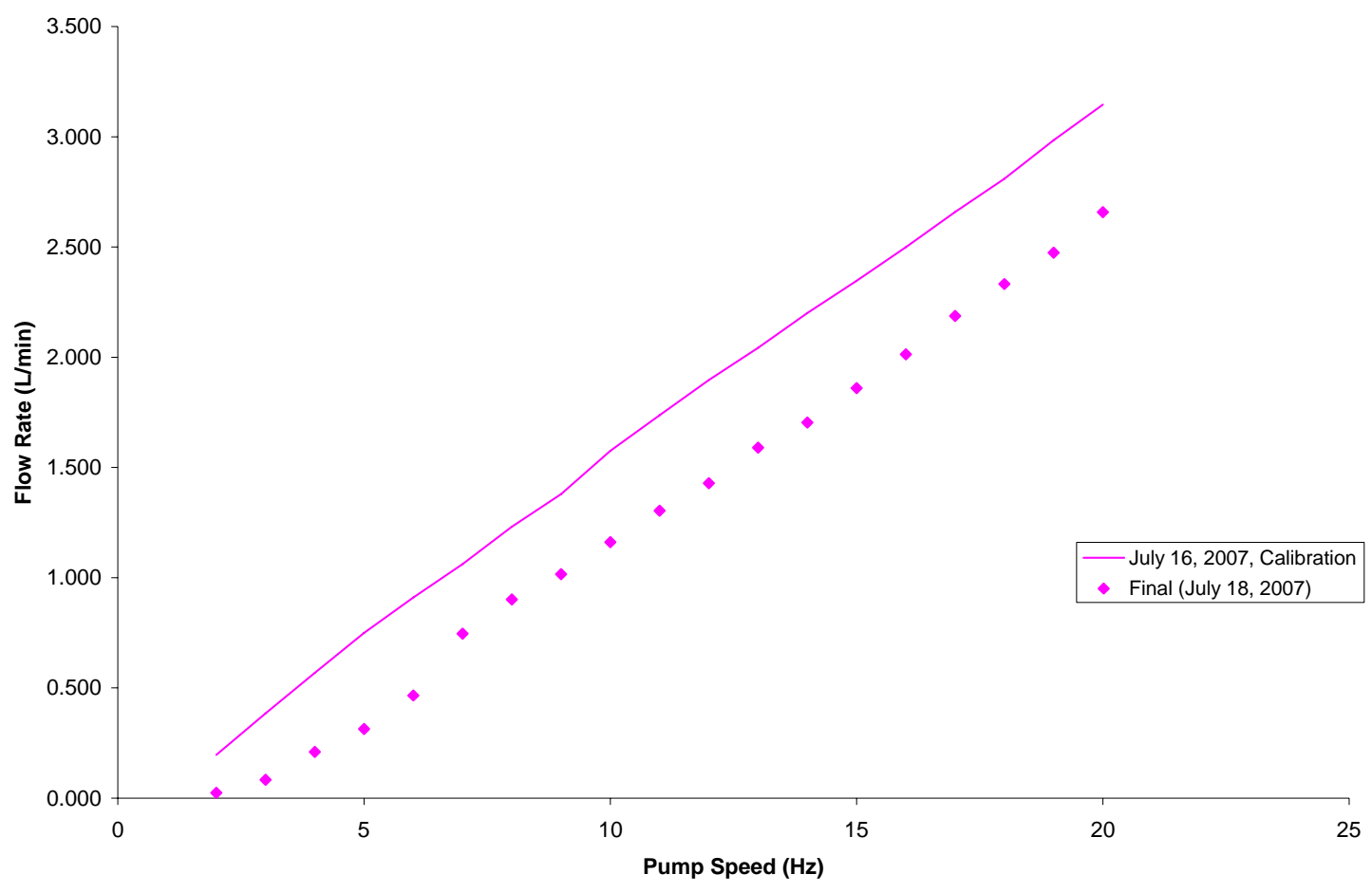

Fig. 23. Final Coriolis flowmeter calibration.

\subsection{FLOW RESPONSE}

Initially both the Coriolis and the magnetic flowmeters were to be tested over the range of flow rates available with this system (pump speed range of $\sim 0-60 \mathrm{~Hz}$ ). This range was based on the initial calibration. After the pump head was replaced, the pressures inside the loop during operation increased so that the pump was allowed to operate only up to $\sim 50 \mathrm{~Hz}$. The flow range tests were partially performed in August 2006 and then delayed until January 2007. Figures 24 and 25 show the flow range responses for the magnetic and the Coriolis meters, respectively. As shown in Fig. 24, the magnetic flowmeter calibrations between August 2006 and January 2007 were slightly different.

However, the flowmeter response was excellent in comparison with the applicable calibration curve. Figure 25 shows that the Coriolis flowmeter calibrations between August 2006 and January 2007 were basically the same and that the flowmeter response was excellent in comparison with the calibration curves. Between 37 and $39.3 \mathrm{~Hz}$, the meter response deviated from the calibration for both the magnetic meter and the Coriolis meter. Since organic entrainment testing was not completed due to failure of the second pump, no flow response tests were conducted for organic-contaminated uranyl nitrate. However, during the calibration with organic, it was noticed that the calibration for volumetric flow rate remained the same. The change in density was noticed on the Coriolis meter, as would be expected. 


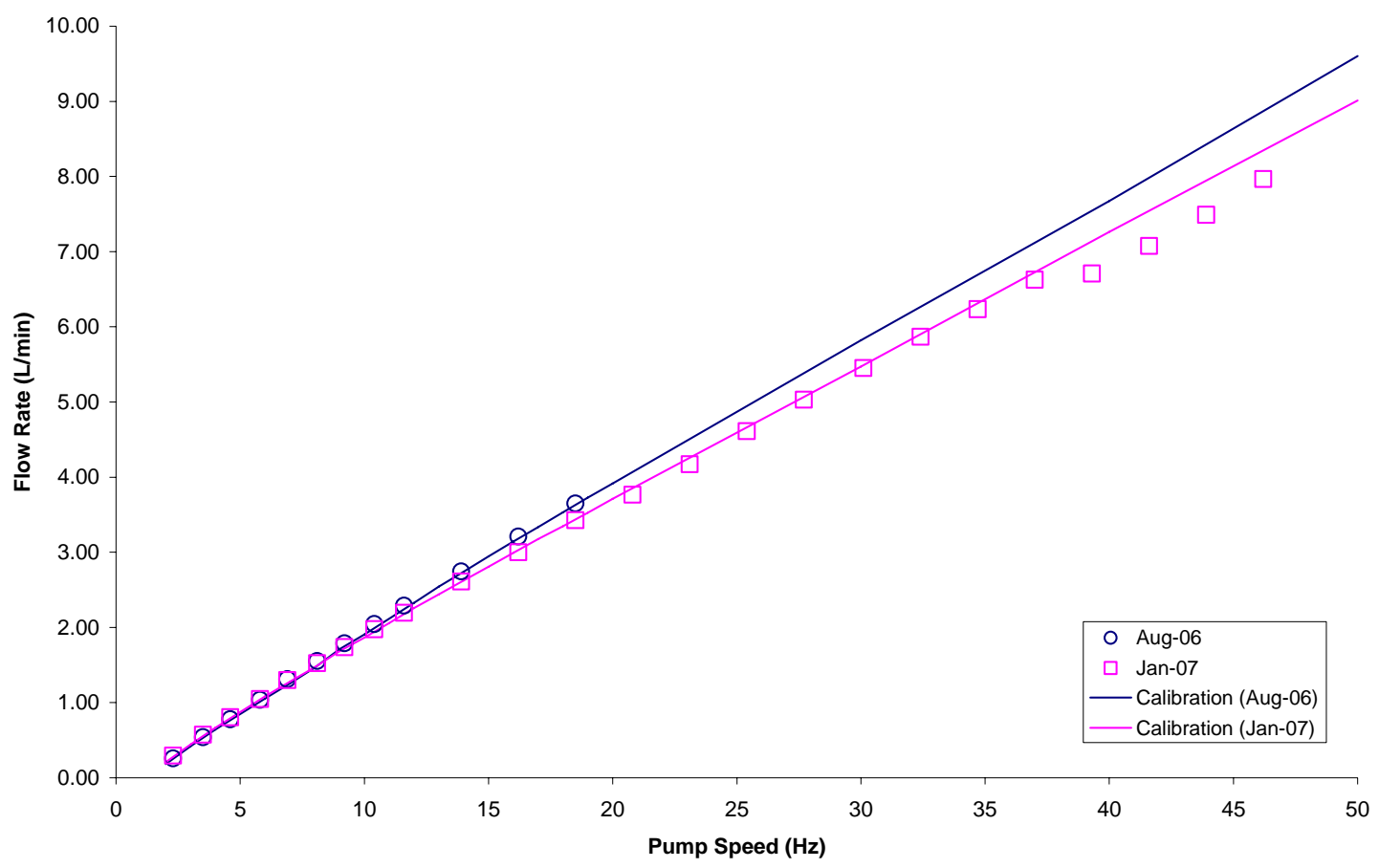

Fig. 24. Magnetic flowmeter response over flow range.

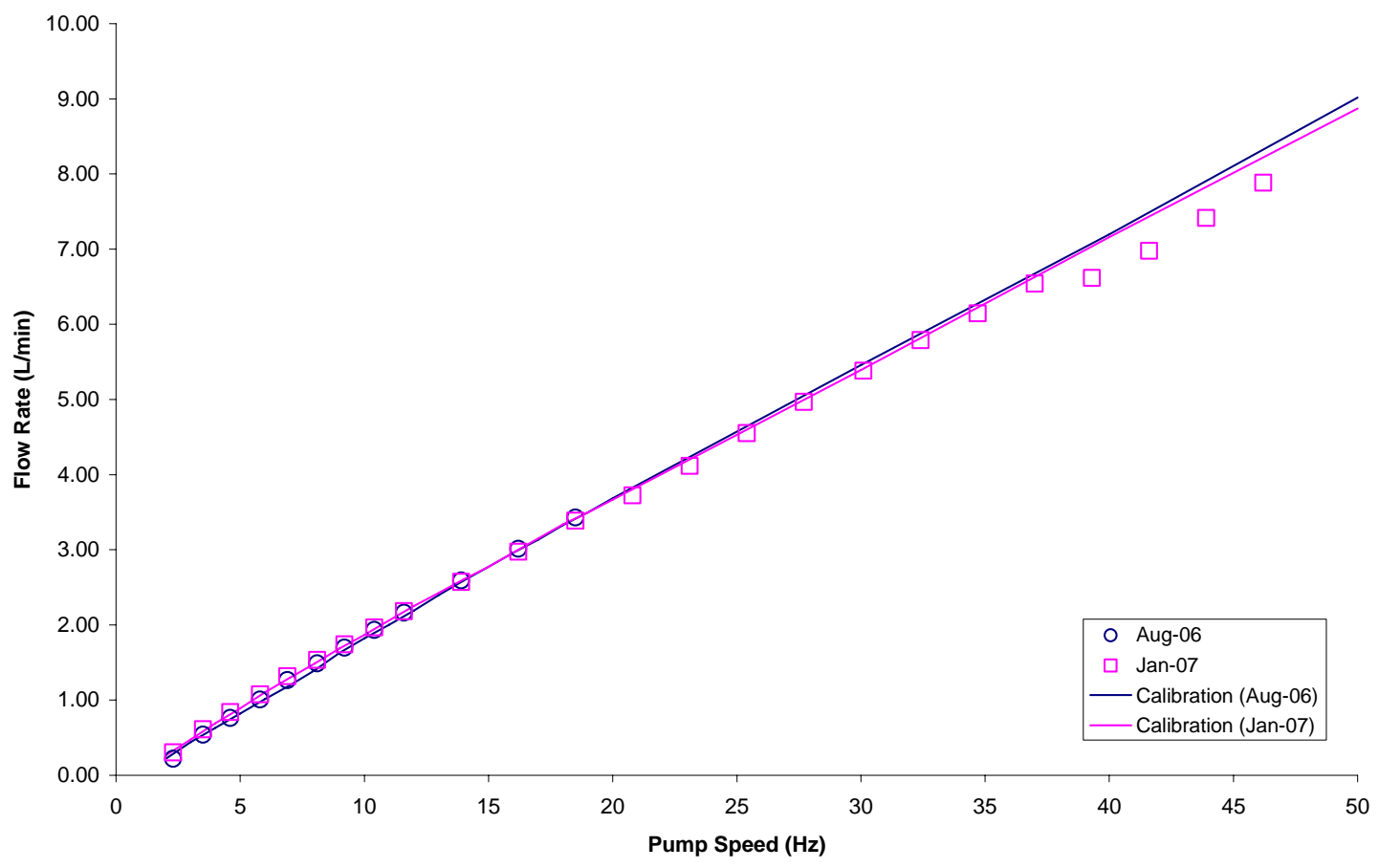

Fig. 25. Coriolis flowmeter response over flow range. 
Flow rates expected at production-scale conversion plants correspond to linear velocities that are achieved in the uranyl nitrate flow loop at low pump speeds $(3.6-11.9 \mathrm{~Hz})$. Figures 26 and 27,

respectively, show the responses of the magnetic and Coriolis flowmeters to low pump speeds. Both meters performed very well in comparison with the calibration curves.

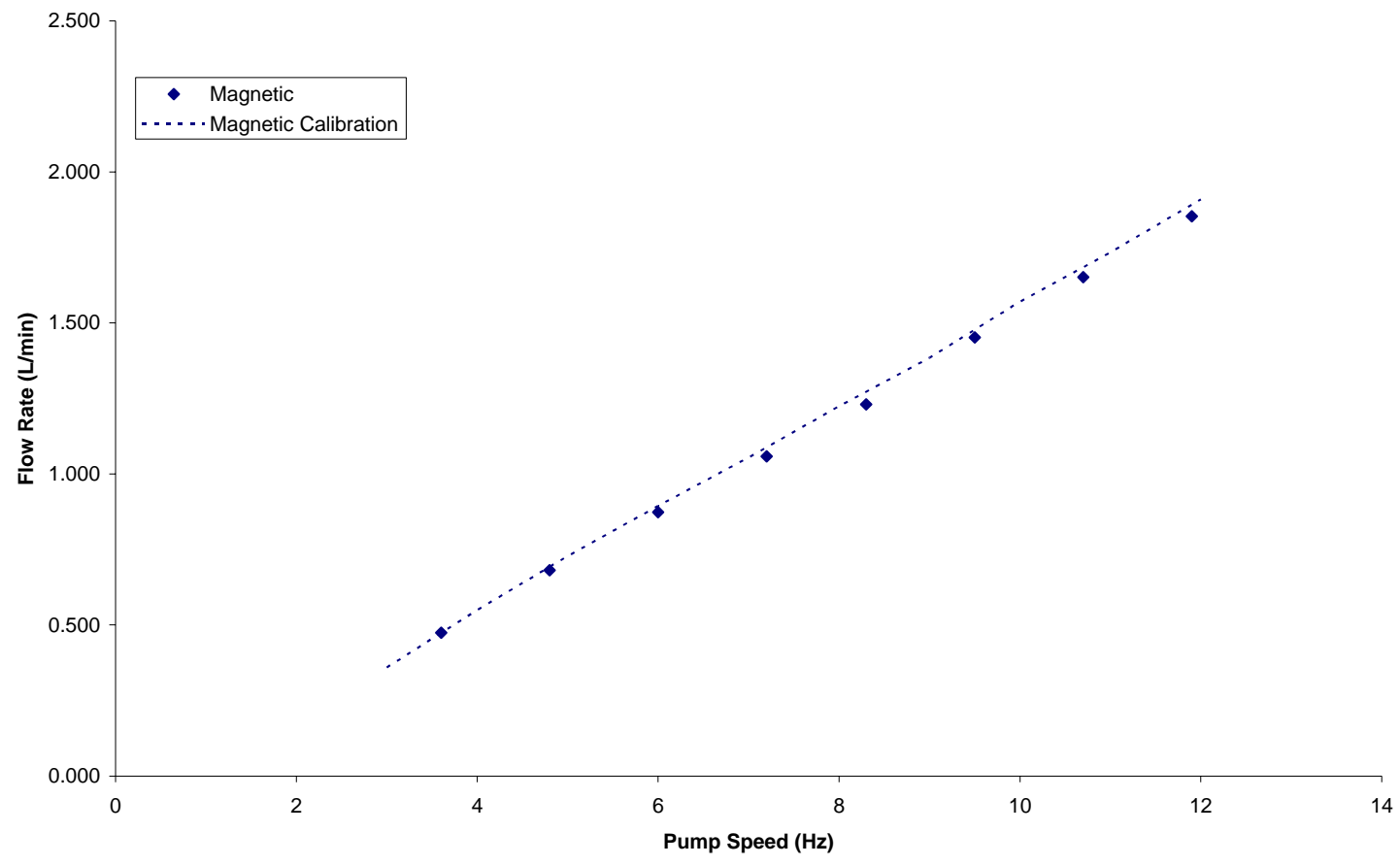

Fig. 26. Magnetic flowmeter response at low pump speeds. 


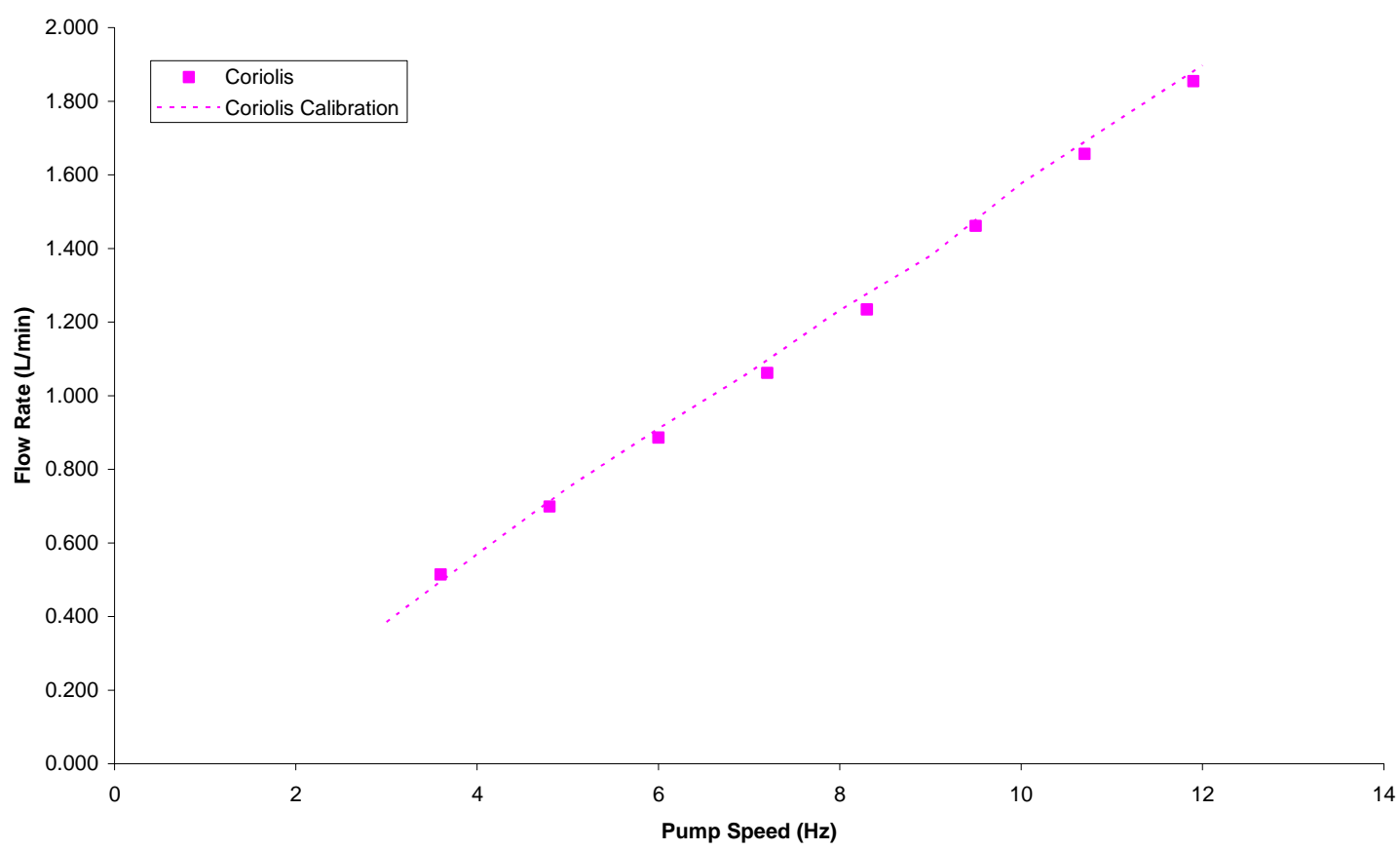

Fig. 27. Coriolis flowmeter response at low pump speeds.

Figures 28-31 show the results of 15 min of steady-state operation at low pump speeds for the two meters. The Coriolis meter had noticeably more perturbations than the magnetic meter. This finding is most likely due to the operating principle but could also be the result of noise. Steady-state operation was also performed for $8 \mathrm{~h}$ to check for zero-drift in the instruments. The results of the 8-h steady-state tests are shown in Table 2, and the corresponding figures are included in Appendix B. As shown in Table 2, the zero-drift for both instruments was negligible over $8 \mathrm{~h}$ of continuous operation. The difference in the meter responses in comparison with the calibration data was good except for pump speeds of $4.8 \mathrm{~Hz}$ for both instruments.

Table 2. Results of $8 \mathrm{~h}$ steady-state flow response tests

\begin{tabular}{ccccc}
\hline \multirow{2}{*}{$\begin{array}{c}\text { Pump speed } \\
\text { (Hz) }\end{array}$} & \multicolumn{2}{c}{ Slope of trend line } & \multicolumn{2}{c}{$\begin{array}{c}\text { Difference from } \\
\text { calibration }\end{array}$} \\
\cline { 2 - 5 } & Magnetic & Coriolis & Magnetic & Coriolis \\
\hline 3.6 & $9.00 \mathrm{E}-06$ & $9.00 \mathrm{E}-06$ & 1.40 & 1.43 \\
4.8 & $-2.00 \mathrm{E}-05$ & $-2.00 \mathrm{E}-05$ & 10.00 & 9.62 \\
6.0 & $4.00 \mathrm{E}-05$ & $4.00 \mathrm{E}-05$ & 4.50 & 6.07 \\
7.2 & $-2.00 \mathrm{E}-05$ & $-2.00 \mathrm{E}-05$ & 3.05 & 2.96 \\
8.3 & $-3.00 \mathrm{E}-05$ & $-3.00 \mathrm{E}-05$ & 1.20 & 0.99 \\
9.5 & $-3.00 \mathrm{E}-05$ & $-4.00 \mathrm{E}-05$ & 0.75 & 0.45 \\
10.7 & $-3.00 \mathrm{E}-05$ & $-2.00 \mathrm{E}-05$ & 2.41 & 2.51 \\
11.9 & $-2.00 \mathrm{E}-05$ & $-2.00 \mathrm{E}-05$ & 3.21 & 3.03 \\
\hline
\end{tabular}




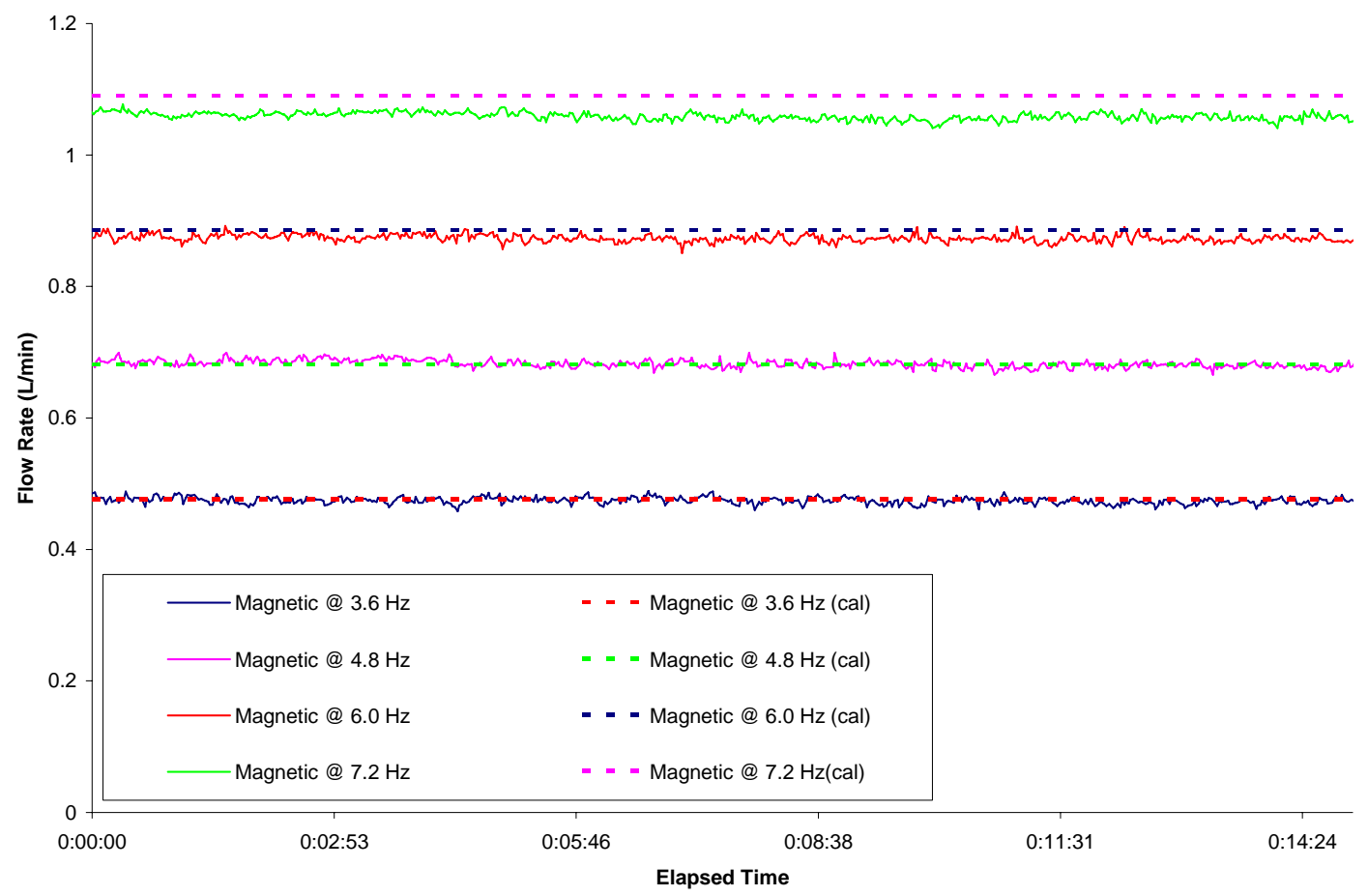

Fig. 28. Magnetic flowmeter response at low pump speeds $(3.6-7.2 \mathrm{~Hz})$ for $15 \mathrm{~min}$.

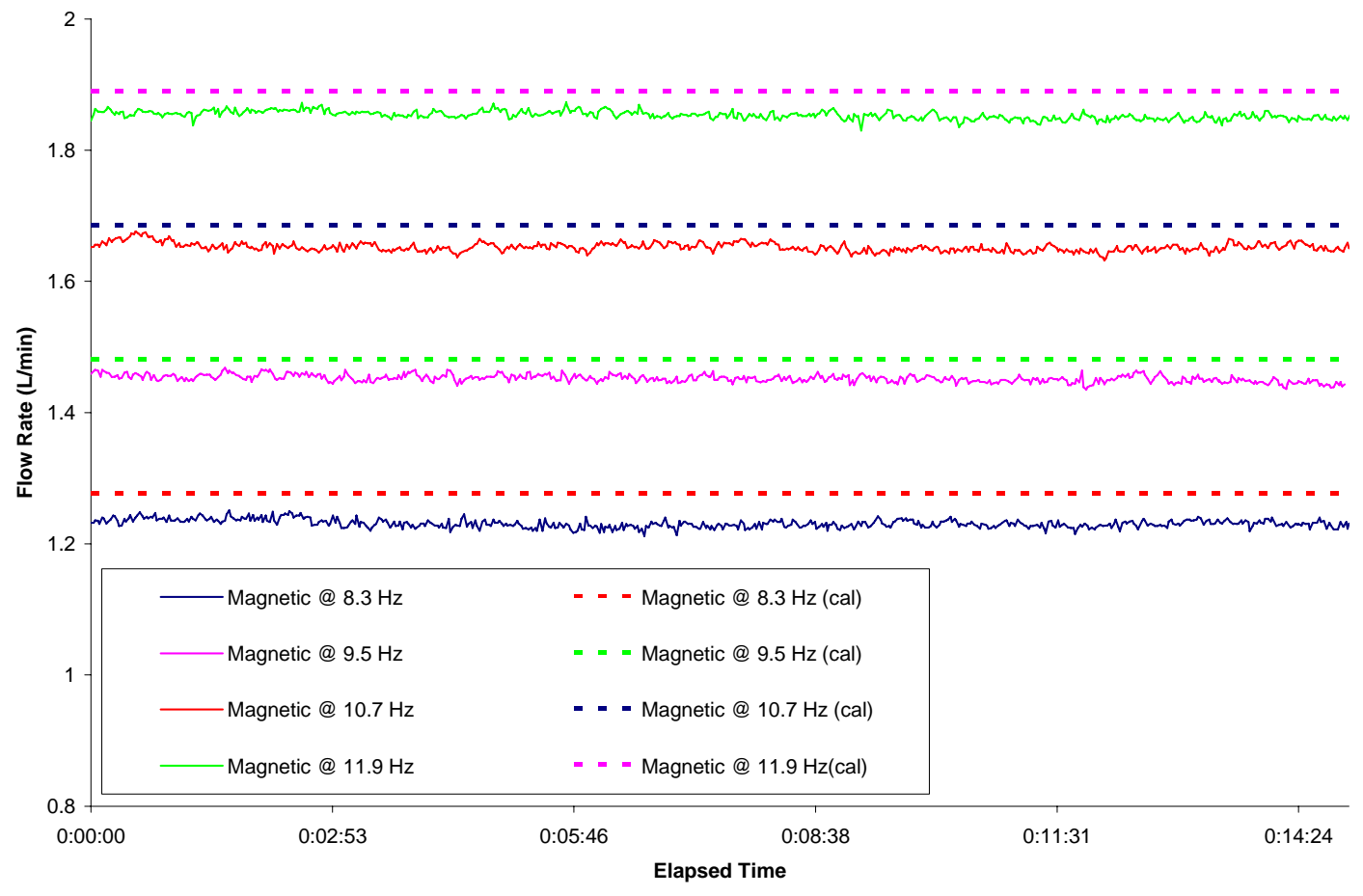

Fig. 29. Magnetic flowmeter response at low pump speeds $(8.3-11.9 \mathrm{~Hz})$ for $15 \mathrm{~min}$. 


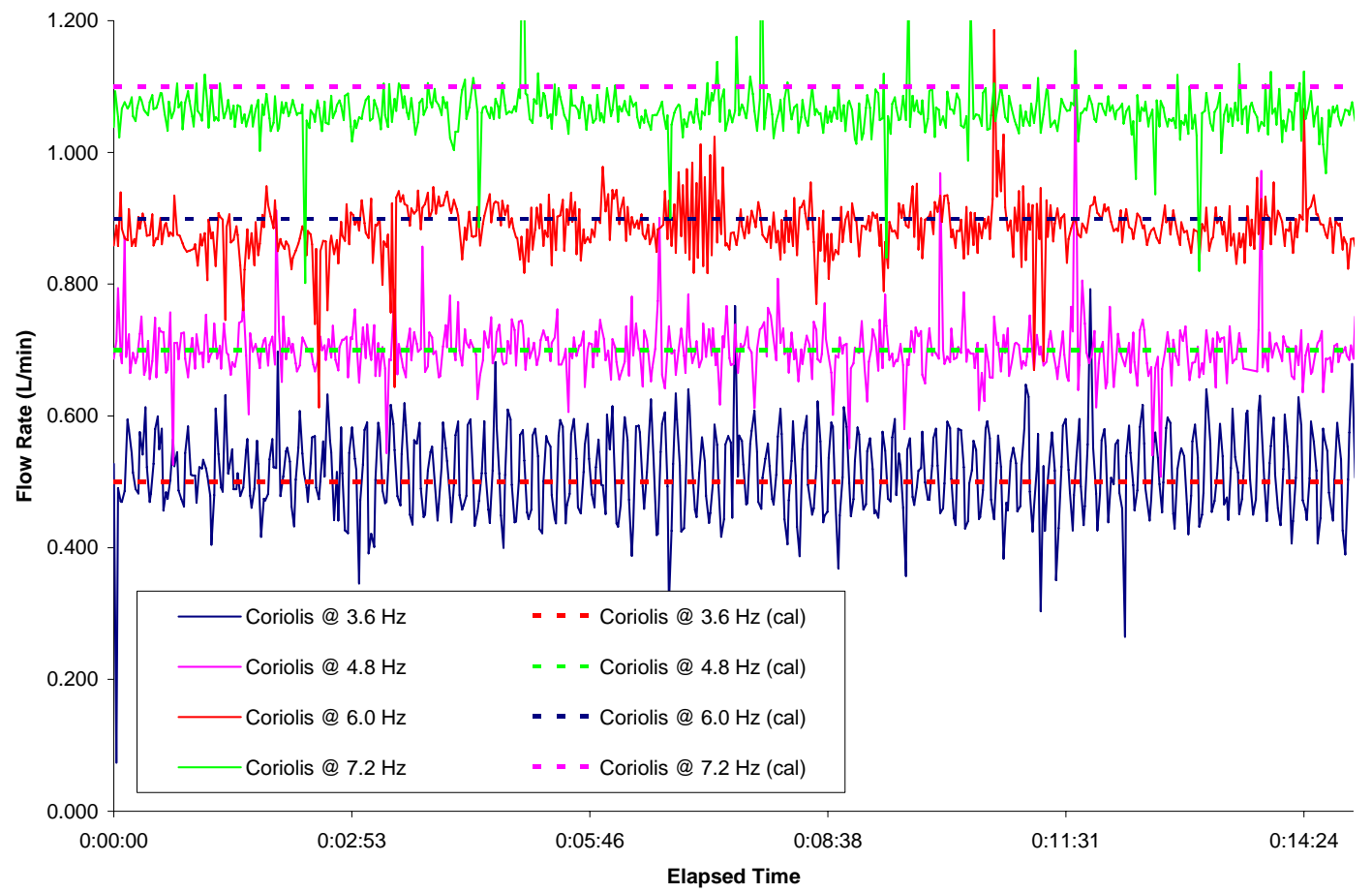

Fig. 30. Coriolis flowmeter response at low pump speeds (3.6-7.2 Hz) for $15 \mathrm{~min}$.

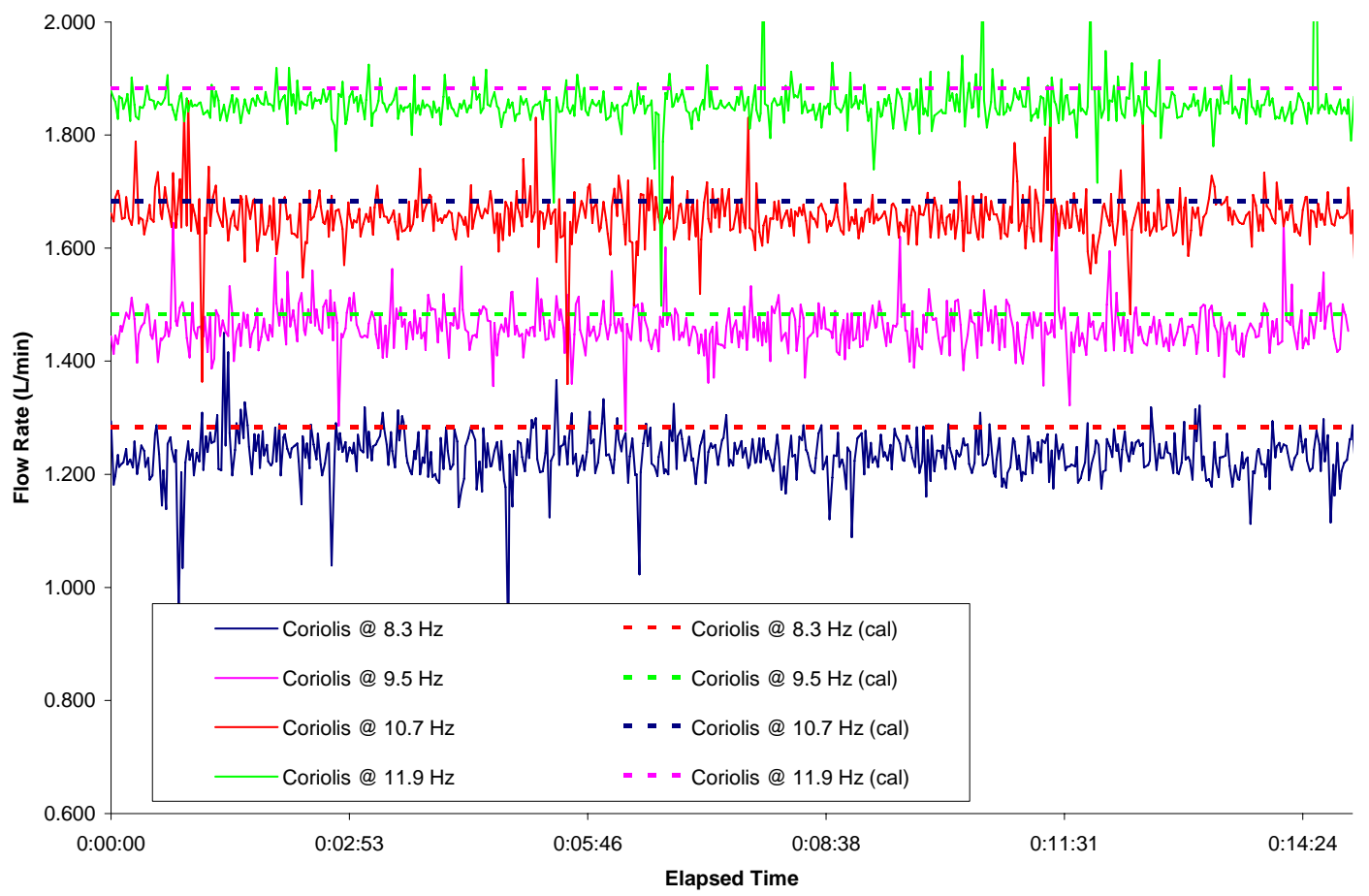

Fig. 31. Coriolis flowmeter response at low pump speeds $(8.3-11.9 \mathrm{~Hz})$ for $15 \mathrm{~min}$. 


\subsection{ENTRAINED AIR RESPONSE}

The entrained air response tests were performed using compressed air that was injected into the system through the air input line (see Fig. 4) at a rate of $500 \mathrm{sccm}$. The air flow rate was held constant throughout each test using a mass flow controller. As a result of air entrainment, the flow rate was reduced noticeably from the expected rate based on the calibration. An example of the results of the entrained air tests is shown in Fig. 32; the remaining figures are included in Appendix C.

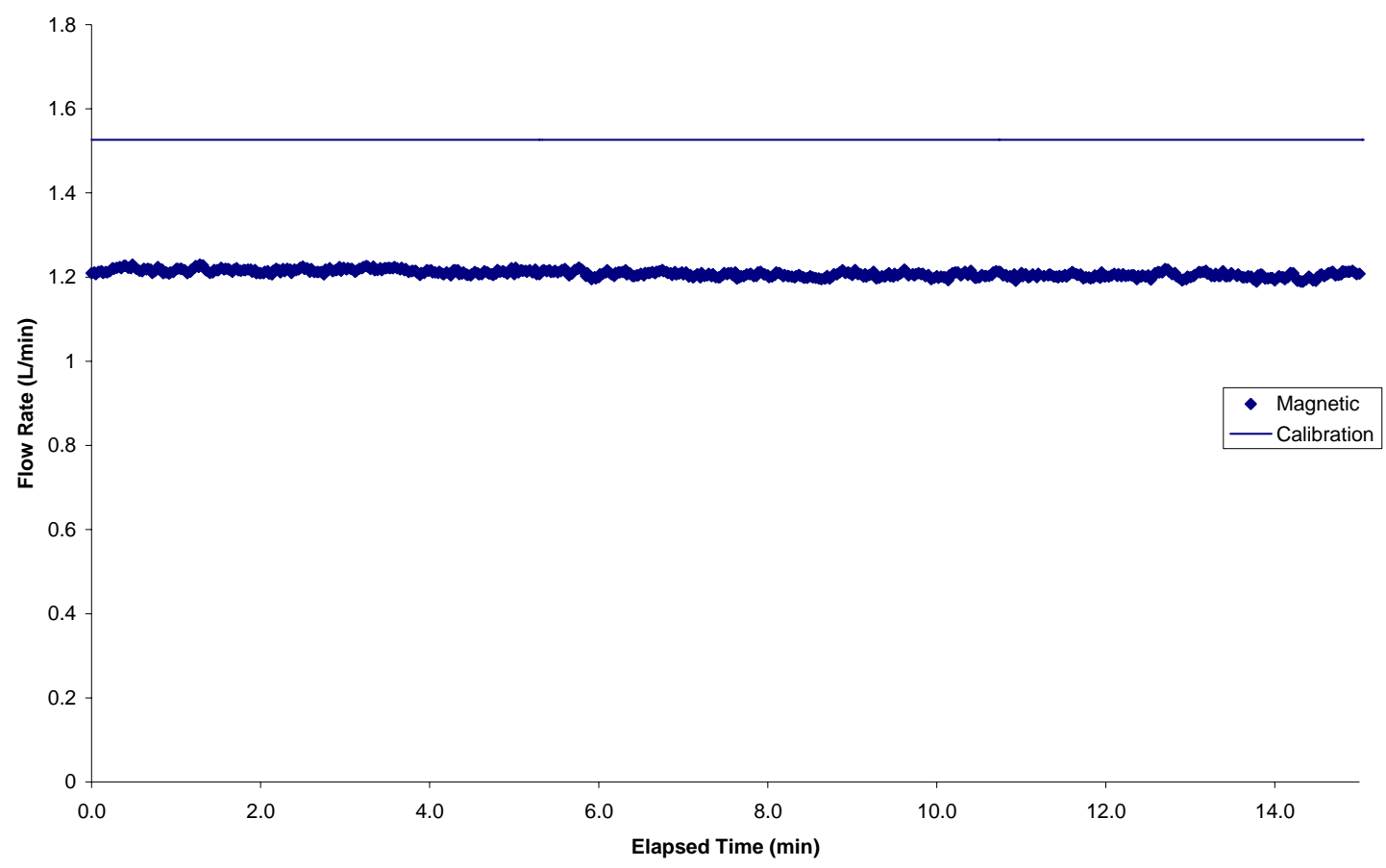

Fig. 32. Magnetic flowmeter response to entrained air $(500 \mathrm{sccm})$ at a pump speed of $8.3 \mathrm{~Hz}$.

\subsection{PULSATING FLOW RESPONSE}

The pulsating flow response tests were performed by manually cycling the pump on and off approximately every $30 \mathrm{~s}$. An example of the result of this type of test is shown in Fig. 33; the remaining figures are included in Appendix D. The result of this operation is an oscillating curve that drops below zero during the "off" position and never reaches full scale based on calibration during the "on" position. 


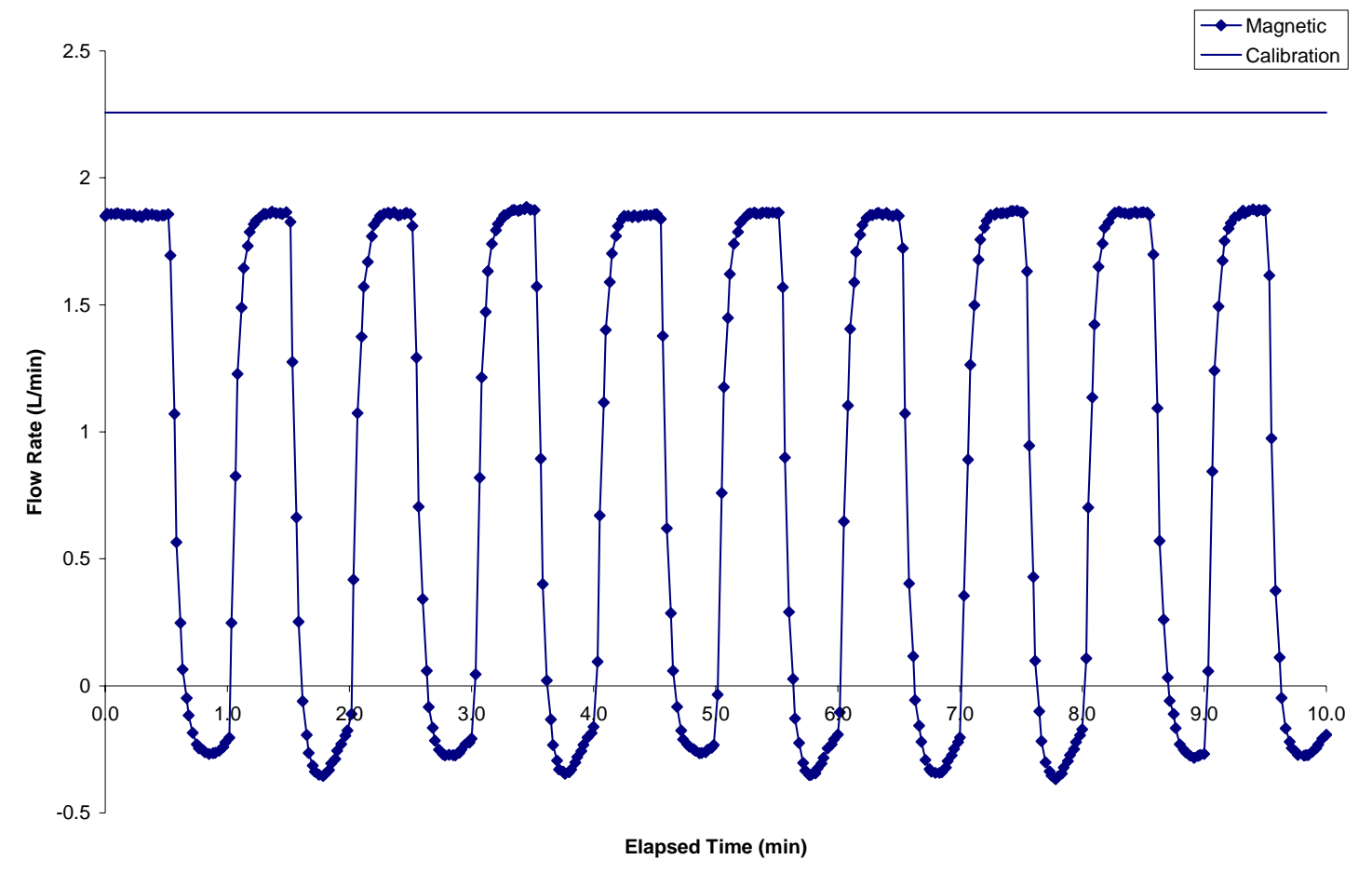

Fig. 33. Magnetic flowmeter response to pulsating flow at a pump speed of $11.9 \mathrm{~Hz}$.

\subsection{PULSATING FLOW WITH ENTRAINED AIR RESPONSE}

The entrained air and pulsating flow tests were combined to determine how the instruments might react to extremes in the process operation. The air flow rate was controlled at $500 \mathrm{sccm}$ and the pump was cycled on and off approximately every minute. For the tests conducted at pump speeds below $8.3 \mathrm{~Hz}$, the curves fluctuated around zero. At $8.3 \mathrm{~Hz}$ and higher, the cycle was noticeable, with the "off" position returning to approximately zero but the "on" position never reaching the calibrated flow rate. Figure 34 shows an example of the results; the remaining figures are included as Appendix E. 


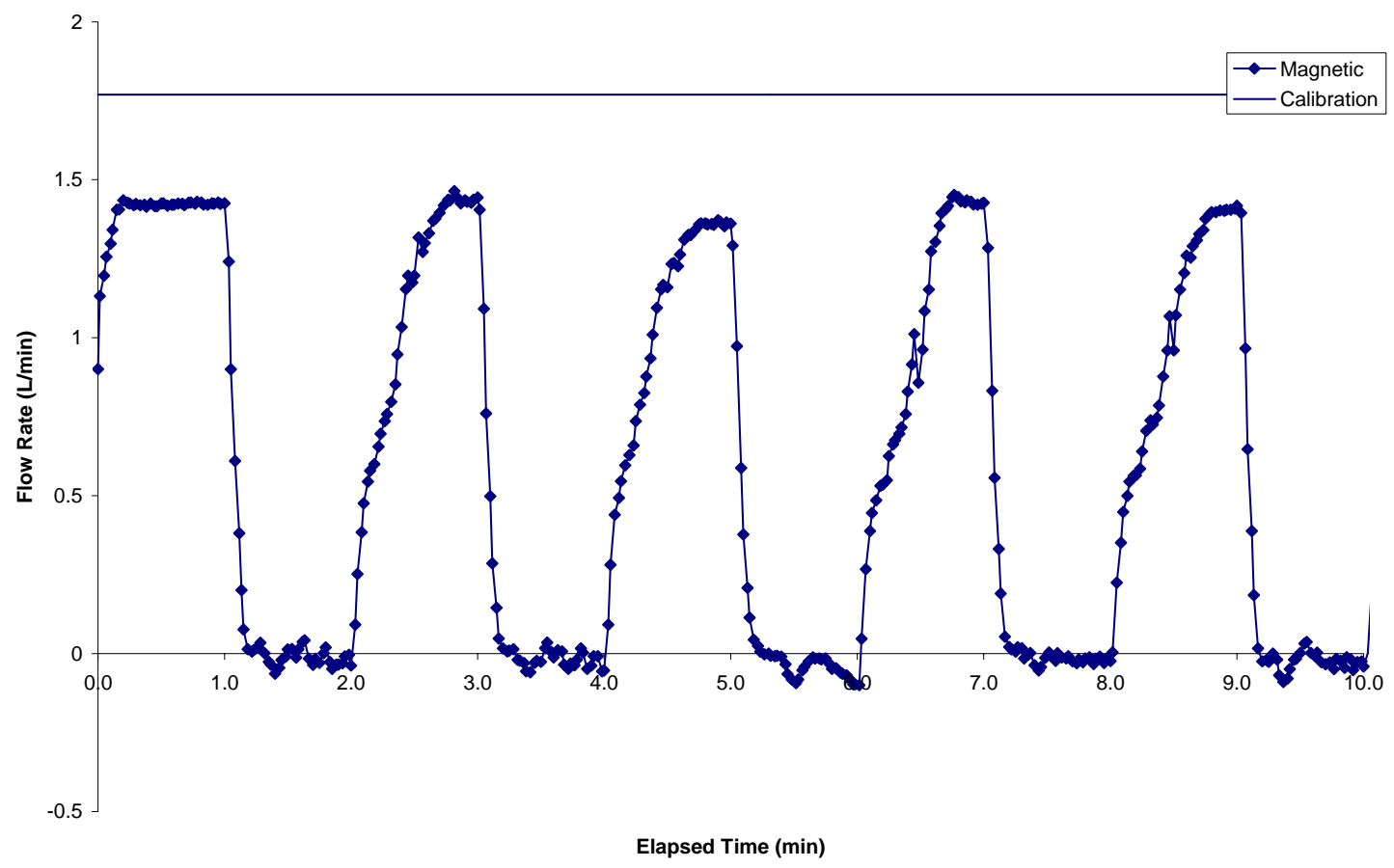

Fig. 34. Magnetic flowmeter response to pulsating flow with entrained air at a pump speed of $9.5 \mathrm{~Hz}$.

\section{CONCLUSIONS}

- The uranyl nitrate flow loop was developed to simulate the purified uranium-bearing aqueous stream exiting the solvent extraction process in an NUCP.

- The loop was used to simulate normal operating conditions and disturbances that would be expected in an NUCP.

- The Coriolis flowmeter and the magnetic flowmeter were found to perform accurately under the conditions expected at an NUCP.

- The ultrasonic flowmeter was not appropriate for these operating conditions.

- Organic-contamination tests were not completed due to pump failure, which occurred possibly as a result of an unexpected incompatibility of gear material with $n$-dodecane or the combination of $n$-dodecane and uranyl nitrate. Pump failure also occurred during testing prior to the addition of organic; again, this is most likely due to an incompatibility of gear material with uranyl nitrate. That is two pump failures occurred during the course of this project, both of which are most likely a result of incompatibility of the pump gear material with uranyl nitrate.

- The zero-drift for the Coriolis meter and the magnetic meter was negligible over $8 \mathrm{~h}$ of continuous testing.

- The flow response of both instruments over the range of flow rates available was linear, as would be expected using a positive displacement pump.

- Air entrainment resulted in a decreased measurement in flow rate for both the Coriolis and the magnetic flowmeters. This is expected because the instruments are not designed to 
measure gas flows. Therefore, since less liquid mass/volume was moving through the system, the liquid flow rate was decreased as indicted by the instrumentation.

- The pulsating flow tests were intended to demonstrate what could occur with the instrument readings during start-up and/or shutdown. As the pump was cycled on and off, the flow rate readings dropped below zero when the pump was turned off and never returned to the calibrated flow rate during the time the pump was running. In an actual plant situation, it would be necessary to determine how long it takes for the flowmeter and flow rate to stabilize after the process is started.

- The tests that included both pulsating flow and entrained air were a worst-case scenario, which is not expected under normal conditions at a production facility at the point in the process that was simulated using the flow loop.

\section{RECOMMENDATIONS}

Because of time constraints on the field testing, it was necessary to recommend a meter for fullscale testing before the small-scale testing had been completed. As a result, the recommendation was based solely on flow response tests performed during August 2005. From this initial testing, it was determined that the ultrasonic meter was inadequate for this application. The ultrasonic flowmeter required that all the fluid properties be specified and that the properties remain unchanged, circumstances that would not be reasonable in an NUCP. The two remaining candidate meters, the Coriolis and the magnetic, were evaluated according to specific selection criteria:

- general industrial applicability and manufacturing quality,

- whether the meter had a pulse output,

- whether the meter had both volume and mass measurement outputs,

- meter specifications and applicability to the process conditions/environment, and

- measurement error of the meter in this application (residual nonlinearity in response). Both of the meters were found to satisfy generally accepted industrial standards for quality and robustness, and both are sufficiently well engineered that either could properly function in a nuclear facility environment. Based on process specifics, a slight preference existed for the Coriolis meter based on overall size and accuracy of measurement and a slight preference existed for the magnetic meter based on measurement range.

Because no preference existed based on the other criteria, the selection of a flowmeter depended on which instrument had the smallest irreducible bias error. Precision was not a substantial contributor to the measurement error for this application, because both instruments had similar levels of precision, and therefore was not considered in the selection process. The irreducible bias error in this case is the nonlinear response of the meters. To measure this nonlinearity, linear regression was used to fit the data and the Pearsons " $\mathrm{R}$ " coefficient was calculated. The variable $R^{2}$ is the variance in the response due to the linear scaling. Therefore, a figure of merit (FOM) was defined as the square root of $1-R^{2}$. This value would represent the residual variance in the response not caused by the linear relationship but would also include variance due to random error. The FOM could be roughly interpreted as the irreducible measurement error due to random error and lack of linearity.

For the tests covering the range of flow rates that could be produced by the pump in this system, the FOMs were 8.7 and $7.4 \%$ for the Coriolis meter and the magnetic meter, respectively. However, when the measurement range was limited to that of only the linear velocities expected at the production-scale facility, the FOM for the Coriolis meter improved to $5.2 \%$ and that for the magnetic meter was 6.0\%. These results are illustrated in Figs. 35 and 36. Based on the FOM over the range of production-scale velocities, the Coriolis meter was slightly preferred over the magnetic meter for production-scale testing. 


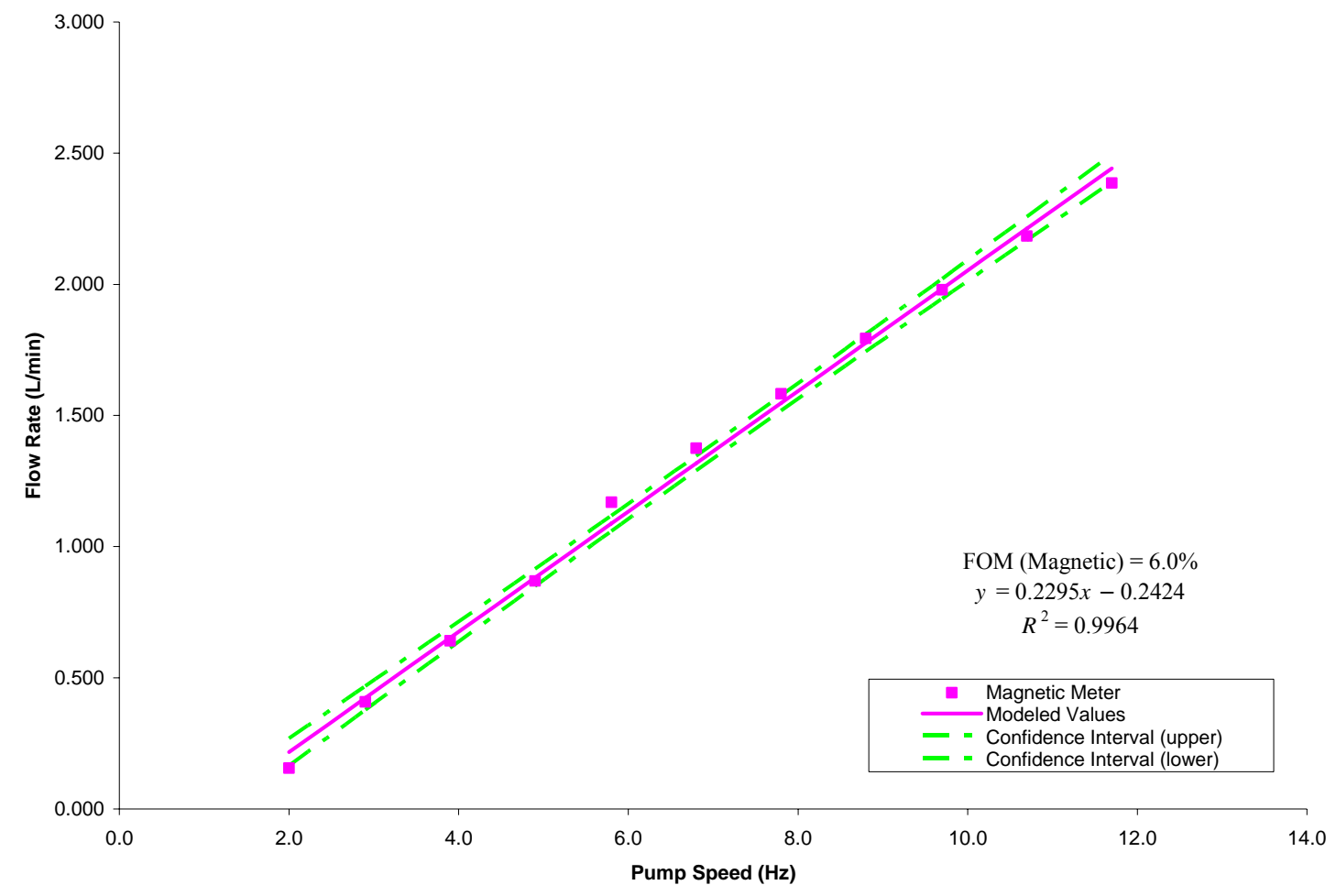

Fig. 35. Magnetic meter response to pump speeds of 2-12 Hz. Note that the flow response is linear with respect to pump speed. The confidence interval bounds are also shown.

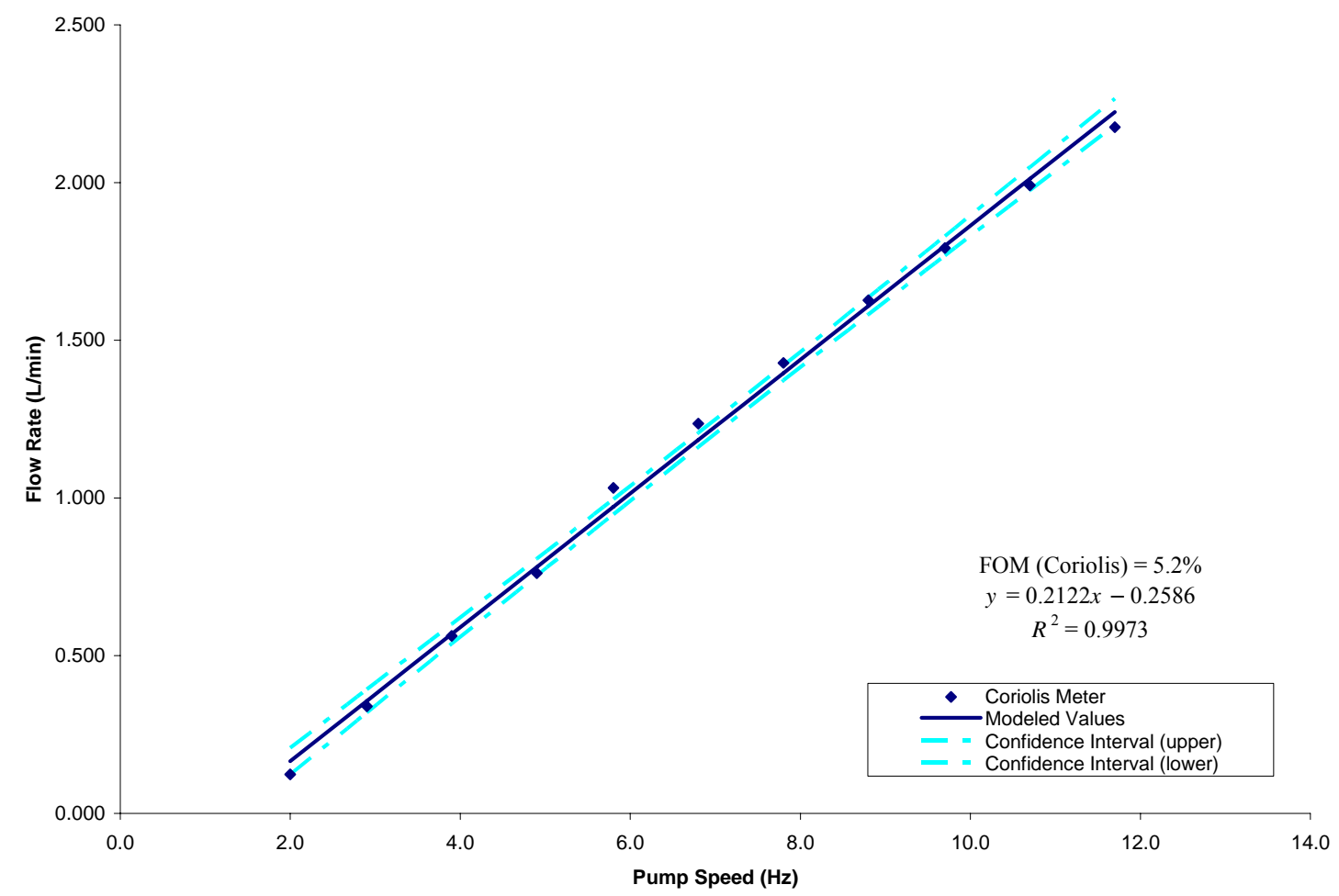

Fig. 36. Coriolis meter response to pump speeds of 2-12 Hz. Note that the flow response is linear with respect to pump speed. The confidence interval bounds are also shown. 
In order to determine the best meter and remove the variance due to random error, it is more accurate to use the mean-squared error (MSE) as the FOM. The MSEs over the range of production-scale velocities were 0.0014 and 0.0022 for the Coriolis meter and the magnetic meter, respectively. Based on the MSE, the Coriolis meter is the best meter for production-scale testing.

Also, a confidence interval calculation was performed on the results for both meters. For the Coriolis meter, the confidence interval on the slope was $0.2048 \leq$ Slope $_{\text {Coriolis }} \leq 0.2196$ and $-0.3142 \leq y$-intercept ${ }_{\text {Coriolis }} \leq-0.2037$ for the $y$-intercept. For the magnetic meter, the confidence interval on the slope was $0.2204 \leq$ Slope $_{\text {Magnetic }} \leq 0.2385$ and $-0.3103 \leq y$-intercept Magnetic $\leq-0.1744$ for the $y$-intercept. The confidence intervals for the flow rate for the magnetic and the Coriolis meters are shown in Figs. 35 and 36, respectively. The negative $y$-intercept is a result of the meter's response not being linear at very low flow rates, i.e. there is some minimum flow rate required before either meter will measure the flow.

Based on the results of the full test series on the small-scale loop, the Coriolis is still the best meter for production-scale applications at NUCPs because of the overall range and accuracy of the measurement. The Coriolis meter also provides a density measurement, which can be helpful in safeguards applications by confirming the uranium concentration throughout the process. Even though the measurement includes more instrument noise (as shown in the figures in the appendices), the inclusion of the density measurement makes the Coriolis meter more difficult to spoof when a material other than uranium is substituted. This feature can prove extremely attractive in safeguards and security operations.

\section{REFERENCES}

1. International Atomic Energy Agency, "The Agency's Safeguards System (1965, as Provisionally Extended in 1966 and 1968)," INFCIRC/66/Rev.2, September 1968, http:/www.iaea.org/Publications/Documents/Infcircs/Others/inf66r2.shtml (July 2008).

2. H. A. Elayat, LLNL, personal communication, 2005.

3. J. Doo, D. Hurt, R. Fagerholm, and N. Tuley, "Safeguards Approach for Natural Uranium Conversion Plants," Proceedings 44th Annual Meeting of Institute of Nuclear Materials Management (INMM), Phoenix, Ariz., July 13-17, 2003.

4. B. D. Boyer, D. M. Gordon, L. G. Fishbone, and J. R. Lemley, "International Safeguards Approach for Uranium Ore Concentrates to Uranium Hexafluoride Conversion Plants," Proceedings 45th Annual Meeting of Institute of Nuclear Materials Management (INMM), Orlando, Fla., July 18-22, 2004.

5. R. L. Faulkner, J. M. Begovich, J. J. Ferrada, R. D. Spence, J. M. Whitaker, W. J. Bicha, and L. G. Loden, "Oak Ridge Efforts to Enhance Conversion Plant Safeguards," Proceedings 45th Annual Meeting of Institute of Nuclear Materials Management (INMM), Orlando, Fla., July 18-22, 2004.

6. R. Faulkner, J. J. Ferrada, L. Loden, R. Spence, and W. Bicha, Natural Uranium Conversion Plant, ORNL/TM-2004/93, Oak Ridge National Laboratory, Oak Ridge, Tenn., April 2004. 
7. International Atomic Energy Agency, "The Structure and Content of Agreements Between the Agency and States Required in Connection with the Treaty on the Non-Proliferation of Nuclear Weapons," INFCIRC/153 (Corrected), June 1972, http://www.fas.org/nuke/control/npt/text/inf153.html (July 2008).

8. A. A. Moghissi, H. W. Godbee, and S. A. Hobert, Radioactive Waste Technology, 1st ed., The American Society of Mechanical Engineers, New York, 1992.

9. J. E. Hardy, J. O. Hylton, T. E. McKnight, C. J. Remenyik, and F. R. Ruppel, Flow Measurement Methods and Applications, 1st ed., John Wiley \& Sons, Inc., New York, 1999.

10. W. L. McCabe, J. C. Smith, and P. Harriott, Unit Operations of Chemical Engineering, 5th ed., McGraw-Hill, New York, 1993.

11. R. H. Perry, D. W. Green, and J. O. Maloney, Perry's Chemical Engineer's Handbook, 7th ed., McGraw-Hill, New York, 1997.

12. E. Loy Upp and Paul J. LaNasa, Fluid Flow Measurement: A Practical Guide to Accurate Flow Measurement, 2nd ed., Gulf Professional Publishing, Boston, 2002. 
APPENDIX A

FLOWMETERS CONSIDERED FOR TESTING 

Table A.1. Summary of specifications for flowmeters considered for testing

\begin{tabular}{|c|c|c|c|c|c|c|c|c|c|c|c|}
\hline Manufacturer & Model no. & Type & $\begin{array}{l}\text { Mounting } \\
\text { type }\end{array}$ & $\begin{array}{l}\text { Measurement } \\
\text { range }\end{array}$ & $\begin{array}{l}\text { Pipe } \\
\text { size } \\
(\mathbf{m m})\end{array}$ & $\begin{array}{c}\% \\
\text { Accuracy }\end{array}$ & $\begin{array}{c}\% \\
\text { Repeatability }\end{array}$ & $\begin{array}{l}\text { Corrosion } \\
\text { resistance }\end{array}$ & Output & Web site & Comments \\
\hline Calibron & Model SVT & Density & Flanged & $0.77-1.4 \mathrm{~g} / \mathrm{cm}^{3}$ & & & 0.01 & SS body & Current & calibron.com & $\begin{array}{l}\text { Volume/mass/ } \\
\text { density }\end{array}$ \\
\hline Calibron & Model 625 & Density & Flanged & $0.77-1.4 \mathrm{~g} / \mathrm{cm}^{3}$ & & & 0.01 & SS body & Current & calibron.com & \\
\hline $\begin{array}{l}\text { Chandler } \\
\text { Engineering }\end{array}$ & $\begin{array}{l}\text { Model } 278 \& \\
303 \text { UGC } \\
\text { vibrating } \\
\text { densitometer }\end{array}$ & Density & Flanged & $0.3-1.6 \mathrm{~g} / \mathrm{cm}^{3}$ & 19 & & & & Current & chandlerengineering.com & $\begin{array}{l}\text { Minimum } \\
\text { flow rate of } \\
5.7 \mathrm{~L} / \mathrm{min} \\
\text { required }\end{array}$ \\
\hline Controlotron & $1010 \mathrm{~N} 1$ & $\begin{array}{l}\text { Ultrasonic- } \\
\text { TT }\end{array}$ & Clamp on & $0-12 \mathrm{~m} / \mathrm{s}$ & $\begin{array}{l}6.4- \\
9144\end{array}$ & $0.5-1$ & 0.15 & N/A & Current & controlotron.com & $\begin{array}{l}\text { Accurate wall } \\
\text { thickness } \\
\text { measurement } \\
\text { improves } \\
\text { accuracy }\end{array}$ \\
\hline Controlotron & System 1020 & $\begin{array}{l}\text { Ultrasonic- } \\
\text { TT }\end{array}$ & Clamp on & $0-12 \mathrm{~m} / \mathrm{s}$ & $12-9150$ & 1 & 0.1 & N/A & Current & controlotron.com & \\
\hline Dynasonics & Series TFXL & $\begin{array}{l}\text { Ultrasonic- } \\
\text { TT }\end{array}$ & Clamp on & $0.03-12.4 \mathrm{~m} / \mathrm{s}$ & $12-2540$ & 1 & & $\mathrm{~N} / \mathrm{A}$ & $\begin{array}{l}\text { Current/ } \\
\text { pulse }\end{array}$ & dynasonics.com & \\
\hline Dynasonics & D301 & $\begin{array}{l}\text { Ultrasonic- } \\
\text { DS }\end{array}$ & Clamp on & $0.03-9 \mathrm{~m} / \mathrm{s}$ & $6-3050$ & 2 & 0.2 & N/A & $\begin{array}{l}\text { Current/ } \\
\text { pulse }\end{array}$ & dynasonics.com & \\
\hline EesiFlo & 6000 Series & $\begin{array}{l}\text { Ultrasonic- } \\
\text { Dual }\end{array}$ & Clamp on & $0.01-25 \mathrm{~m} / \mathrm{s}$ & $6 \&$ up & $1.0-3.0$ & 0.15 & N/A & $\begin{array}{l}\text { Current/ } \\
\text { pulse/ freq }\end{array}$ & eesiflo.com & Portable \\
\hline EesiFlo & 7000 Series & $\begin{array}{l}\text { Ultrasonic- } \\
\text { Dual }\end{array}$ & Clamp on & $0.01-25 \mathrm{~m} / \mathrm{s}$ & $6 \&$ up & $1.0-3.0$ & 0.15 & N/A & Current & eesiflo.com & Fixed \\
\hline $\begin{array}{l}\text { Emerson Process } \\
\text { Management } \\
\text { (Brooks } \\
\text { Instruments) }\end{array}$ & Quantim Series & Coriolis & Threaded & $50-5,000 \mathrm{~g} / \mathrm{h}$ & $1.6-6$ & 0.5 & 0.05 & SS body & Current & emersonprocess.com & $\begin{array}{l}\text { Volume/mass/ } \\
\text { density/ } \\
\text { temperature }\end{array}$ \\
\hline $\begin{array}{l}\text { Emerson Process } \\
\text { Management } \\
\text { (Micro Motion) }\end{array}$ & $\begin{array}{l}\text { Elite- } \\
\text { CMF050M } 313 \\
\text { N A B U E Z Z } \\
\text { Z }\end{array}$ & Coriolis & Flanged & $0-3,400 \mathrm{~L} / \mathrm{h}$ & $12-25$ & 0.1 & 0.05 & SS body & Current & emersonprocess.com & $\begin{array}{l}\text { Volume/mass/ } \\
\text { density/ } \\
\text { temperature }\end{array}$ \\
\hline $\begin{array}{l}\text { Emerson Process } \\
\text { Management } \\
\text { (Micro Motion) }\end{array}$ & $\begin{array}{l}\text { Elite- } \\
\text { CMF100H }\end{array}$ & Coriolis & Flanged & $0-13,600 \mathrm{~L} / \mathrm{h}$ & $25-50$ & 0.1 & 0.05 & SS body & Current & emersonprocess.com & $\begin{array}{l}\text { Volume/mass/ } \\
\text { density/ } \\
\text { temperature }\end{array}$ \\
\hline
\end{tabular}


Table A.1 (cont.). Summary of specifications for flowmeters considered for testing

\begin{tabular}{|c|c|c|c|c|c|c|c|c|c|c|c|}
\hline Manufacturer & Model no. & Type & $\begin{array}{c}\text { Mounting } \\
\text { type }\end{array}$ & $\begin{array}{l}\text { Measurement } \\
\text { range }\end{array}$ & $\begin{array}{l}\text { Pipe } \\
\text { size } \\
(\mathrm{mm})\end{array}$ & $\begin{array}{c}\% \\
\text { Accuracy }\end{array}$ & $\begin{array}{c}\% \\
\text { Repeatability }\end{array}$ & $\begin{array}{l}\text { Corrosion } \\
\text { resistance }\end{array}$ & Output & Web site & Comments \\
\hline $\begin{array}{l}\text { Emerson Process } \\
\text { Management } \\
\text { (Rosemount) }\end{array}$ & $\begin{array}{l}8700 \text { Series } \\
(8732 \\
\text { transmitter } \\
\text { with } 8705 \text { flow } \\
\text { tube) }\end{array}$ & Magnetic & Flanged & $0.01-10 \mathrm{~m} / \mathrm{s}$ & $15-900$ & 0.5 & 0.1 & $\begin{array}{l}\text { SS with } \\
\text { Teflon } \\
\text { lining }\end{array}$ & Current & emersonprocess.com & \\
\hline Endress + Hauser & Promass $40 \mathrm{E}$ & Coriolis & Flanged & $0-70,000 \mathrm{~kg} / \mathrm{h}$ & $8-50$ & $0.5-0.7$ & $0.25-0.35$ & & $\begin{array}{l}\text { Current/ } \\
\text { pulse/ freq }\end{array}$ & endress.com & $\begin{array}{l}\text { Volume/mass/ } \\
\text { temperature }\end{array}$ \\
\hline Endress+Hauser & $\begin{array}{l}\text { Promass } \\
80 / 83 \mathrm{H}\end{array}$ & Coriolis & Flanged & $0-70,000 \mathrm{~kg} / \mathrm{h}$ & $8-50$ & $0.15-0.25$ & $0.05-0.2$ & & $\begin{array}{c}\text { Current/ } \\
\text { pulse/ freq }\end{array}$ & endress.com & $\begin{array}{l}\text { Volume/mass/ } \\
\text { density/ } \\
\text { temperature }\end{array}$ \\
\hline Endress+Hauser & $\begin{array}{l}\text { Prosonic 90/93 } \\
\text { W/U }\end{array}$ & $\begin{array}{l}\text { Ultrasonic- } \\
\text { TT }\end{array}$ & Clamp on & $0-15 \mathrm{~m} / \mathrm{s}$ & $15-4000$ & 0.5 & 0.3 & N/A & $\begin{array}{l}\text { Current/ } \\
\text { pulse/ freq }\end{array}$ & endress.com & 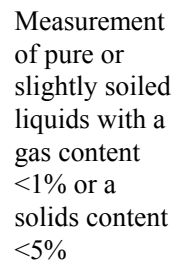 \\
\hline GE-Panametrics & DF868 & $\begin{array}{l}\text { Ultrasonic- } \\
\text { TT/Dual }\end{array}$ & Clamp on & $0.03-12.2 \mathrm{~m} / \mathrm{s}$ & $12-5100$ & $2-5$ & $0.2-0.5$ & N/A & Current & gepower.com & Low accuracy \\
\hline GE-Panametrics & XMT868 & $\begin{array}{l}\text { Ultrasonic- } \\
\text { TT/Dual }\end{array}$ & Clamp on & $0.03-12.2 \mathrm{~m} / \mathrm{s}$ & $12-5100$ & 2 & $0.2-0.5$ & N/A & Current & gepower.com & Low accuracy \\
\hline GE-Panametrics & UTX878 & $\begin{array}{l}\text { Ultrasonic- } \\
\text { TT }\end{array}$ & Clamp on & $0.03-12.2 \mathrm{~m} / \mathrm{s}$ & $12-203$ & $2-5$ & $0.1-0.3$ & N/A & Current & gepower.com & Low accuracy \\
\hline Hellma, GmbH & & $\begin{array}{c}\text { Fiber-optic } \\
\text { probes }\end{array}$ & & & & & & & & hellma-worldwide.de & \\
\hline $\begin{array}{l}\text { King Instrument } \\
\text { Co. }\end{array}$ & 7100 Series & & In-line & $0.6-95 \mathrm{~L} / \mathrm{h}$ & & 5 & 1 & & & kinginstrumentco.com & $\begin{array}{l}\text { Low accuracy } \\
\text { for low flow }\end{array}$ \\
\hline Krohne & UFM 3030 & $\begin{array}{l}\text { Ultrasonic- } \\
\text { TT }\end{array}$ & Flanged & $0-20 \mathrm{~m} / \mathrm{s}$ & $25-300$ & 0.5 & 0.2 & SS body & $\begin{array}{c}\text { Pulse/ } \\
\text { freq }\end{array}$ & krohne-mar.com & $\begin{array}{l}\text { Handles up to } \\
5 \% \text { solids and } \\
2 \% \text { entrained } \\
\text { air, optional } \\
\text { mass flow } \\
\text { output }\end{array}$ \\
\hline
\end{tabular}


Table A.1 (cont.). Summary of specifications for flowmeters considered for testing

\begin{tabular}{|c|c|c|c|c|c|c|c|c|c|c|c|}
\hline Manufacturer & Model no. & Type & $\begin{array}{c}\text { Mounting } \\
\text { type }\end{array}$ & $\begin{array}{l}\text { Measurement } \\
\text { range }\end{array}$ & $\begin{array}{c}\text { Pipe } \\
\text { size } \\
(\mathrm{mm})\end{array}$ & $\begin{array}{c}\% \\
\text { Accuracy }\end{array}$ & $\begin{array}{c}\% \\
\text { Repeatability }\end{array}$ & $\begin{array}{l}\text { Corrosion } \\
\text { resistance }\end{array}$ & Output & Web site & Comments \\
\hline $\begin{array}{l}\text { LC Liquid } \\
\text { Controls }\end{array}$ & $\begin{array}{l}\text { LCMass RHM } \\
\text { Type F/G }\end{array}$ & Coriolis & $\begin{array}{l}\text { Flanged/ } \\
\text { Threaded }\end{array}$ & $\begin{array}{c}0.002-30,000 \\
\mathrm{~kg} / \mathrm{min}\end{array}$ & $6-300$ & 0.2 & 0.1 & $316 \mathrm{Ti} \mathrm{SS}$ & & lcmeter.com & \\
\hline Oceanoptics & & $\begin{array}{l}\text { Fiber-optic } \\
\text { probes }\end{array}$ & & & & & & & & oceanoptics.com & \\
\hline $\begin{array}{l}\text { Princo } \\
\text { Instruments }\end{array}$ & Densitrol & Density & & & & & & & & princoinstruments.com & \\
\hline $\begin{array}{l}\text { Proteus } \\
\text { Industries Inc }\end{array}$ & 200 Series & Turbine & Threaded & $0.4-230 \mathrm{~L} / \mathrm{min}$ & & 3 & & SS body & Voltage & proteusind.com & Low accuracy \\
\hline $\begin{array}{l}\text { Proteus } \\
\text { Industries Inc }\end{array}$ & 500 Series & Turbine & Threaded & $0.6-17 \mathrm{~L} / \mathrm{min}$ & & 2 & 0.5 & SS body & Voltage & proteusind.com & Low accuracy \\
\hline $\begin{array}{l}\text { Proteus } \\
\text { Industries Inc }\end{array}$ & $\begin{array}{l}\text { FluidVision } \\
4000\end{array}$ & Turbine & Threaded & $0.3-230 \mathrm{~L} / \mathrm{min}$ & 12 & 3 & 1 & SS body & $\begin{array}{l}\text { Current/ } \\
\text { voltage }\end{array}$ & proteusind.com & $\begin{array}{l}\text { Flow/ } \\
\text { temperature/ } \\
\text { pressure }\end{array}$ \\
\hline $\begin{array}{l}\text { Proteus } \\
\text { Industries Inc }\end{array}$ & 800 Series & Turbine & Threaded & $0.2-190 \mathrm{~L} / \mathrm{min}$ & & 2 & 0.5 & SS body & Voltage & proteusind.com & Low accuracy \\
\hline $\begin{array}{l}\text { Ronan } \\
\text { Engineering }\end{array}$ & & Density & & & & & & & & ronanmeasure.com & $\begin{array}{l}\text { Uses } \\
\text { cesium-137 }\end{array}$ \\
\hline SeaMetrics & SEB-075 & Turbine & Threaded & $0.8-38 \mathrm{~L} / \mathrm{min}$ & 19 & 1 & & & & seametrics.com & \\
\hline SeaMetrics & EX80 & Magnetic & & $0.06-6.09 \mathrm{~m} / \mathrm{s}$ & $25-200$ & 1 & & SS body & $\begin{array}{l}\text { Square } \\
\text { wave } \\
\text { pulse }\end{array}$ & seametrics.com & \\
\hline Sparling & FS-555 & $\begin{array}{l}\text { Ultrasonic- } \\
\text { TT }\end{array}$ & & & & 1 & & & Current & sparlinginstruments.com & \\
\hline ThermoElectron & DCT1088 & $\begin{array}{l}\text { Ultrasonic- } \\
\text { TT }\end{array}$ & Clamp on & $0.0-15 \mathrm{~m} / \mathrm{s}$ & $25 \&$ up & 0.5 & 0.2 & N/A & Current & thermo.com & \\
\hline ThermoElectron & TX10 & $\begin{array}{l}\text { Ultrasonic- } \\
\text { TT }\end{array}$ & Clamp on & $0-12 \mathrm{~m} / \mathrm{s}$ & $25-500$ & 1 & & N/A & Current & thermo.com & \\
\hline TTI Inc & Time Delta M & $\begin{array}{l}\text { Ultrasonic- } \\
\text { TT }\end{array}$ & Clamp on & $0-10 \mathrm{~m} / \mathrm{s}$ & $25-225$ & 0.5 & 0.5 & N/A & Current & ttiglobal.com & \\
\hline
\end{tabular}


Table A.1 (cont.). Summary of specifications for flowmeters considered for testing

\begin{tabular}{|c|c|c|c|c|c|c|c|c|c|c|c|}
\hline Manufacturer & Model no. & Type & $\begin{array}{c}\text { Mounting } \\
\text { type }\end{array}$ & $\begin{array}{l}\text { Measurement } \\
\text { range }\end{array}$ & $\begin{array}{c}\text { Pipe } \\
\text { size } \\
(\mathbf{m m})\end{array}$ & $\begin{array}{c}\% \\
\text { Accuracy }\end{array}$ & $\begin{array}{c}\% \\
\text { Repeatability }\end{array}$ & $\begin{array}{l}\text { Corrosion } \\
\text { resistance }\end{array}$ & Output & Web site & Comments \\
\hline TTI Inc & CompuFlow & $\begin{array}{l}\text { Ultrasonic- } \\
\text { DS }\end{array}$ & Clamp on & $0.02-15.25 \mathrm{~m} / \mathrm{s}$ & $12-7620$ & 2 & 0.1 & N/A & Current & ttiglobal.com & $\begin{array}{l}\text { High solid } \\
\text { content, } \\
\text { maintenance } \\
\text { free }\end{array}$ \\
\hline TTI Inc & Time Delta S & $\begin{array}{l}\text { Ultrasonic- } \\
\text { TT }\end{array}$ & Clamp on & $0.018-32 \mathrm{~m} / \mathrm{s}$ & $12-6000$ & $0.5-2$ & 0.5 & N/A & Current & ttiglobal.com & \\
\hline Yokogawa & ADMAG & Magnetic & Flanged & $0.1-10 \mathrm{~m} / \mathrm{s}$ & $2.5-200$ & 0.5 & 0.1 & SS body & & us.yokogawa.com & \\
\hline Yokogawa & ADMAG AE & Magnetic & Flanged & $0.3-10 \mathrm{~m} / \mathrm{s}$ & $2.5-400$ & $0.1-0.25$ & 0.1 & SS body & Current & us.yokogawa.com & \\
\hline Yokogawa & ADMAG CA & Magnetic & Flanged & $0.5-10 \mathrm{~m} / \mathrm{s}$ & $15-200$ & $0.5-1$ & 0.1 & $\begin{array}{l}\text { Alumina } \\
\text { ceramics } \\
(99.9 \%)\end{array}$ & Current & us.yokogawa.com & \\
\hline Yokogawa & $\begin{array}{l}\text { ADMAG } \\
\text { SE115 M J A } \\
\text { A1S L }\end{array}$ & Magnetic & Flanged & $0.3-10 \mathrm{~m} / \mathrm{s}$ & $12-400$ & 0.5 & 0.1 & SS body & Current & us.yokogawa.com & \\
\hline Yokogawa & DY-D, DY-E & Vortex & Flanged & $10 \mathrm{~m} / \mathrm{s}(\max )$ & $15-300$ & 0.75 & 0.2 & SS body & Current & us.yokogawa.com & $\begin{array}{l}5.26-\mathrm{L} / \mathrm{min} \\
\text { cutoff }\end{array}$ \\
\hline Yokogawa & $\begin{array}{l}\text { ROTAMASS } \\
\text { 3-Series }\end{array}$ & Coriolis & Flanged & $0.3-5 \mathrm{~kg} / \mathrm{L}$ & & 0.1 & & SS body & $\begin{array}{l}\text { Current/ } \\
\text { pulse }\end{array}$ & us.yokogawa.com & \\
\hline
\end{tabular}


APPENDIX B

GRAPHS OF 8-h STEADY-STATE FLOW 



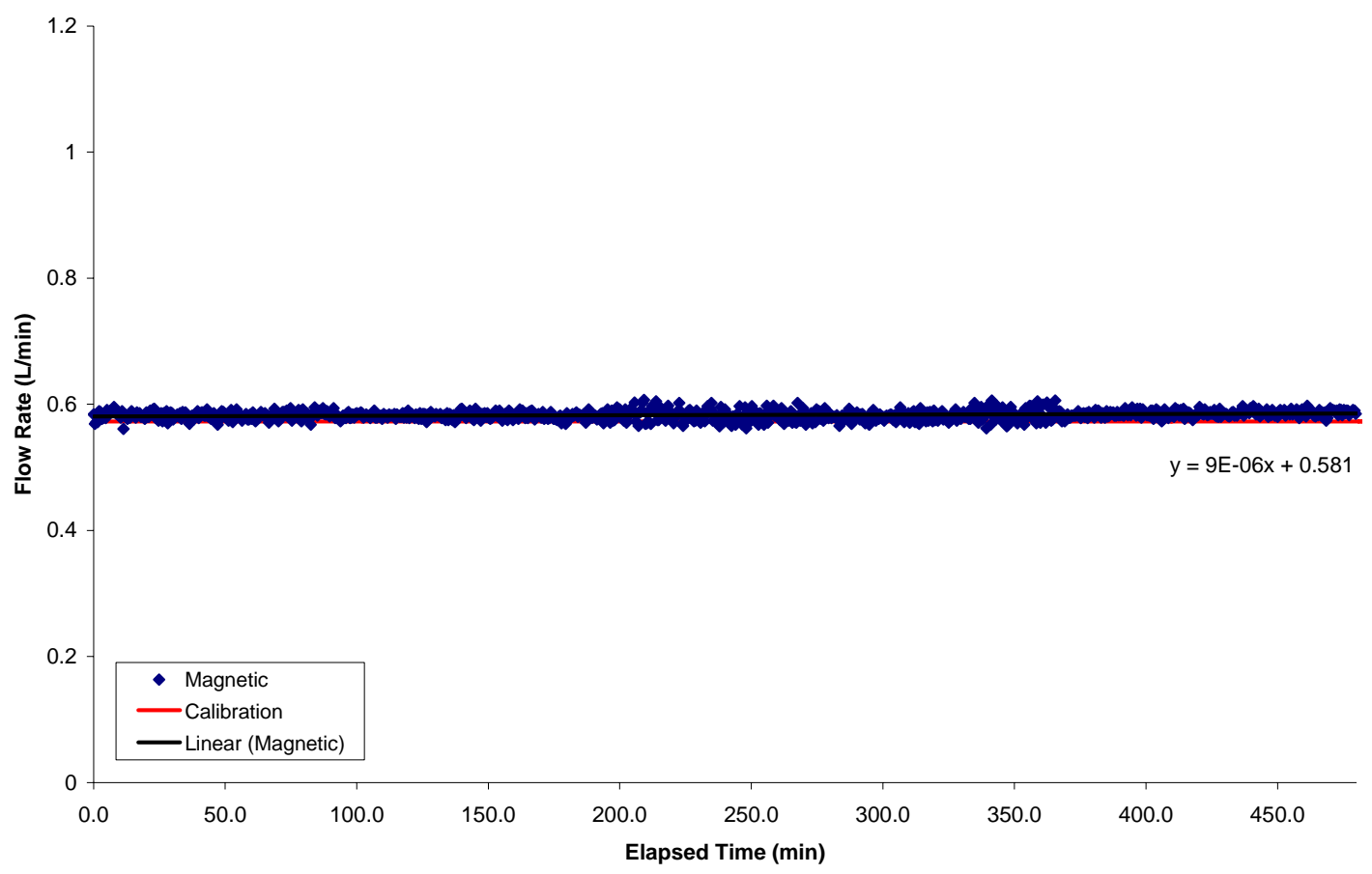

Fig. B.1. Magnetic response to steady-state flow at $3.6 \mathrm{~Hz}$ for 8 -h continuous operation.

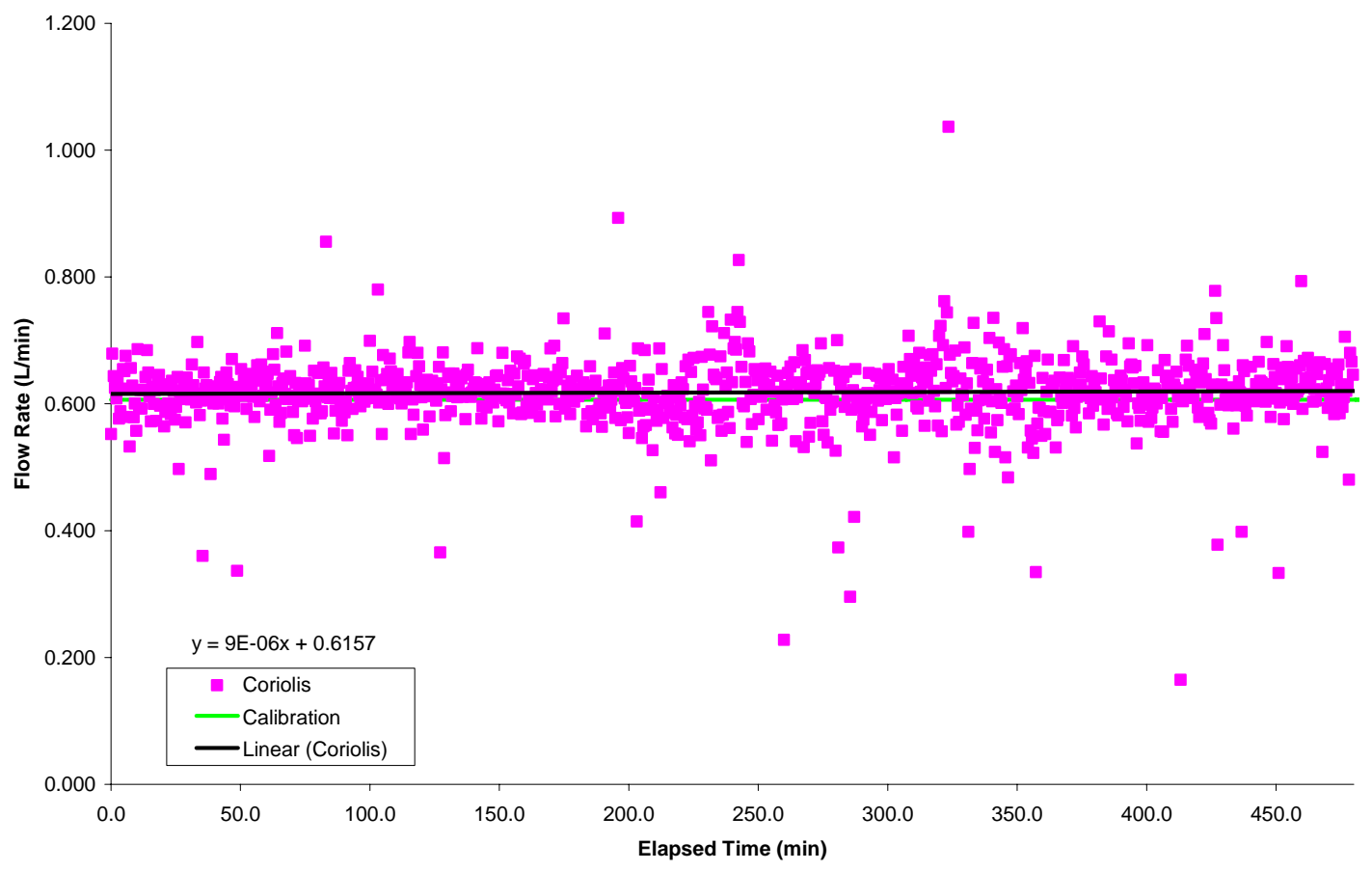

Fig. B.2. Coriolis response to steady-state flow at $3.6 \mathrm{~Hz}$ for 8 -h continuous operation. 


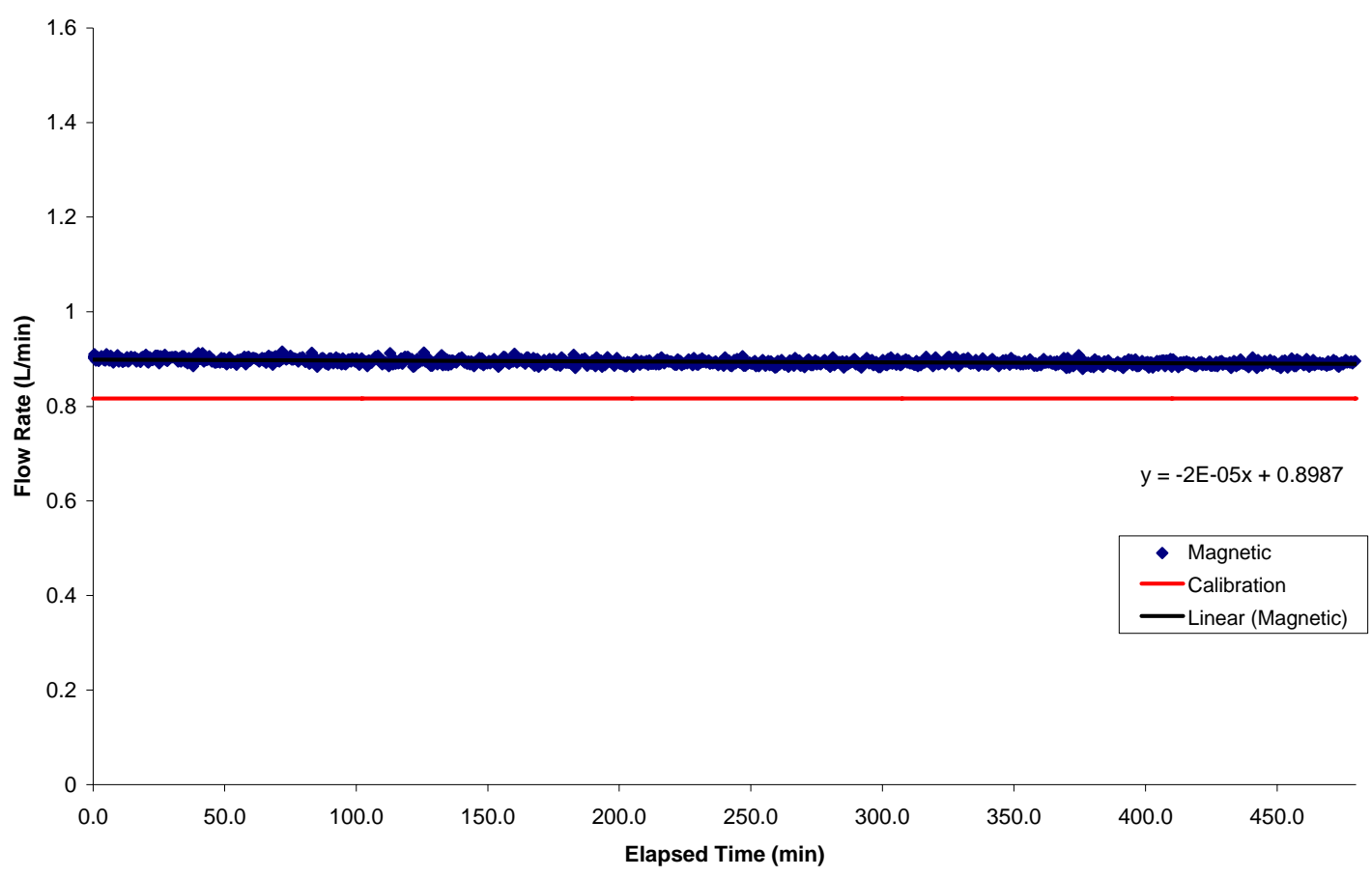

Fig. B.3. Magnetic response to steady-state flow at $4.8 \mathrm{~Hz}$ for 8 -h continuous operation.

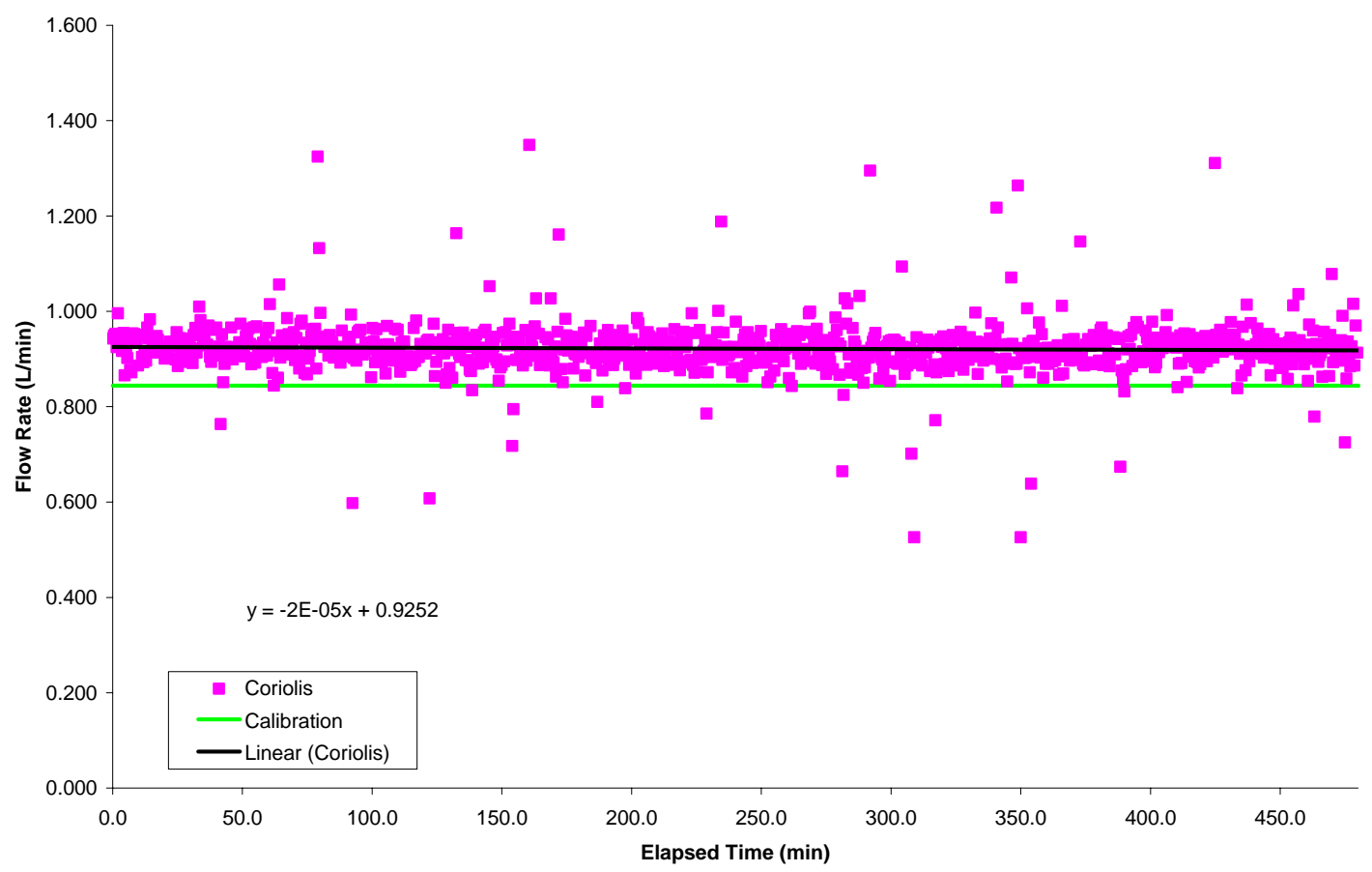

Fig. B.4. Coriolis response to steady-state flow at $4.8 \mathrm{~Hz}$ for 8 -h continuous operation. 


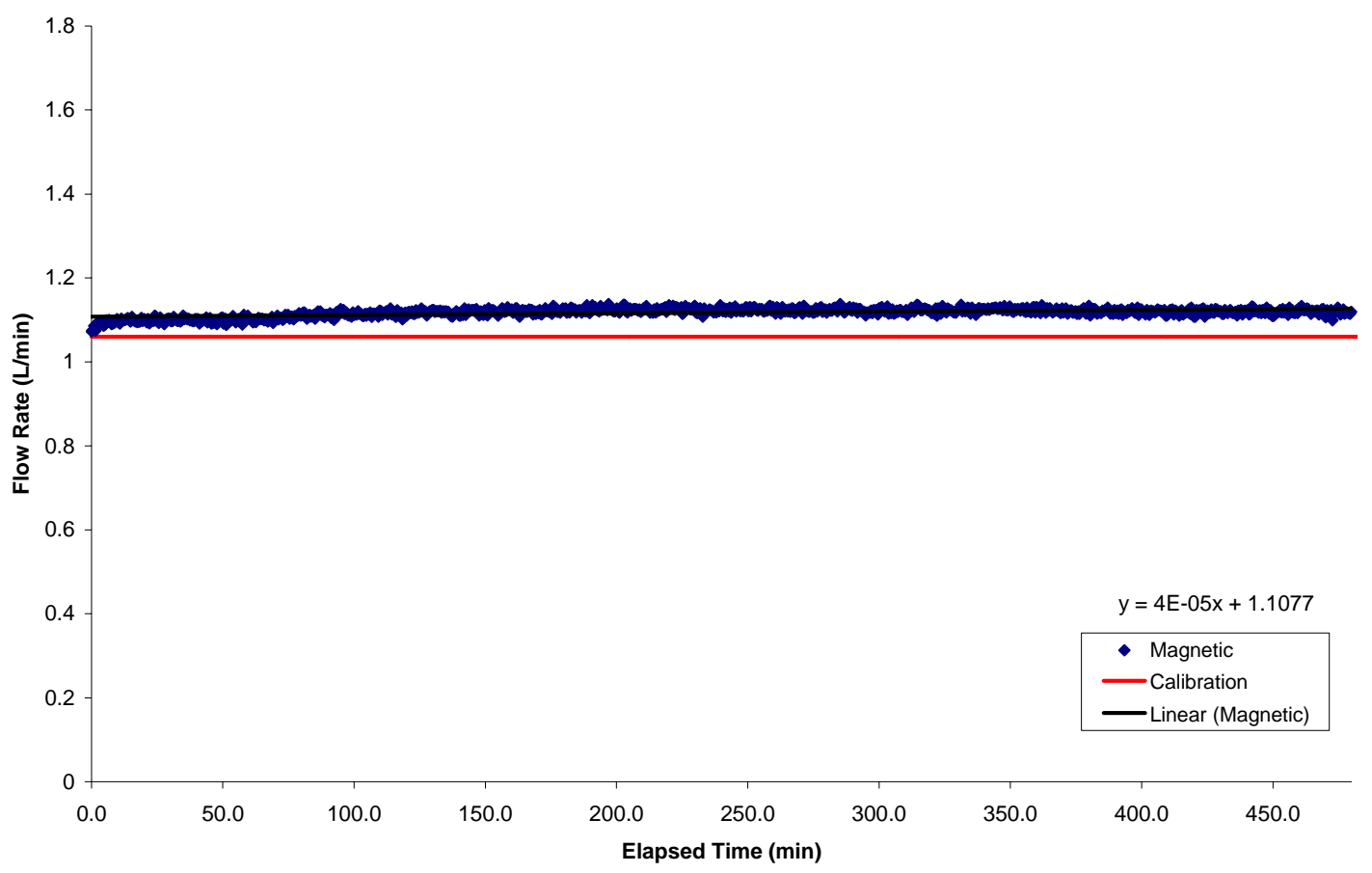

Fig. B.5. Magnetic response to steady-state flow at $6.0 \mathrm{~Hz}$ for 8 -h continuous operation.

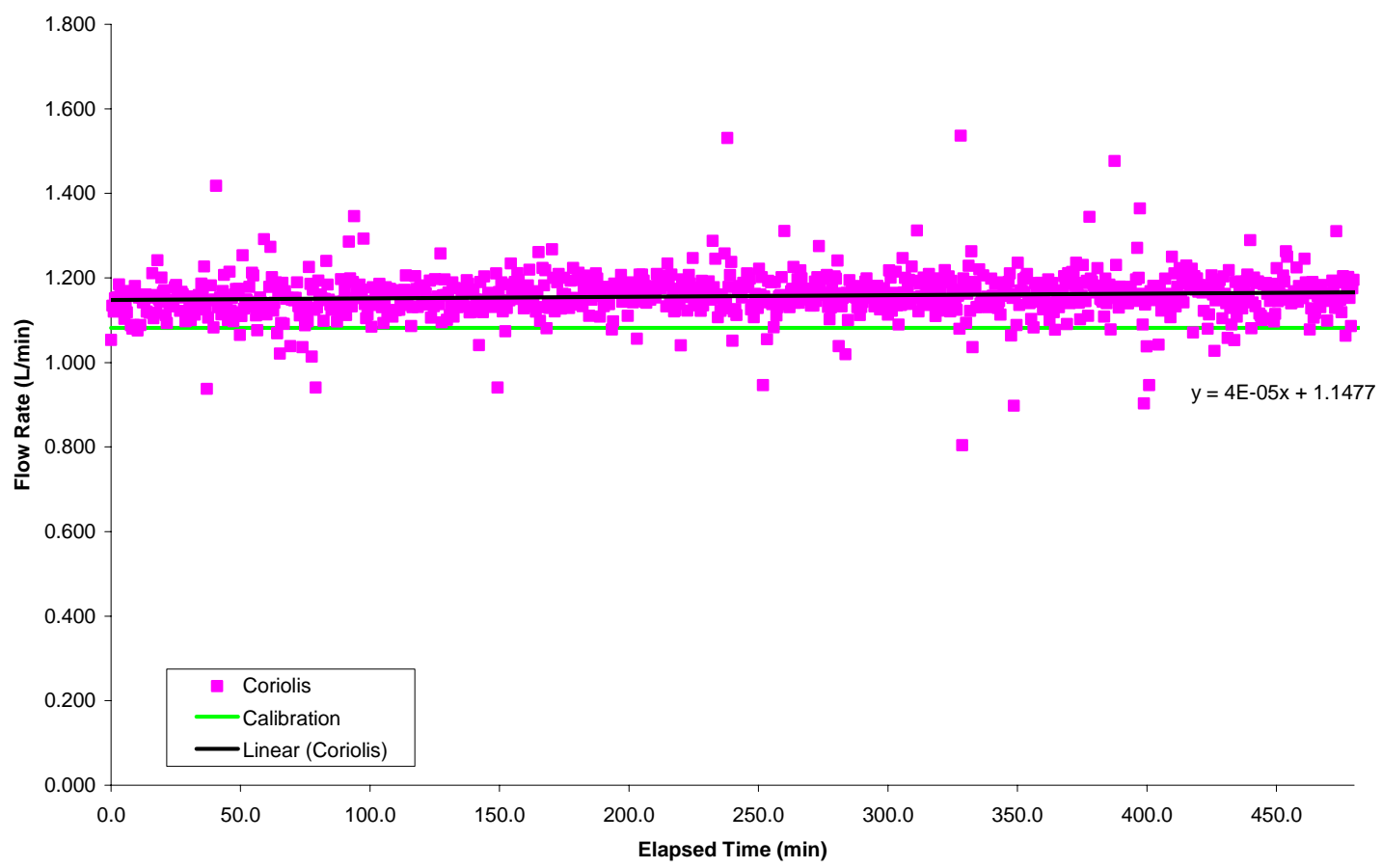

Fig. B.6. Coriolis response to steady-state flow at $6.0 \mathrm{~Hz}$ for 8 -h continuous operation. 


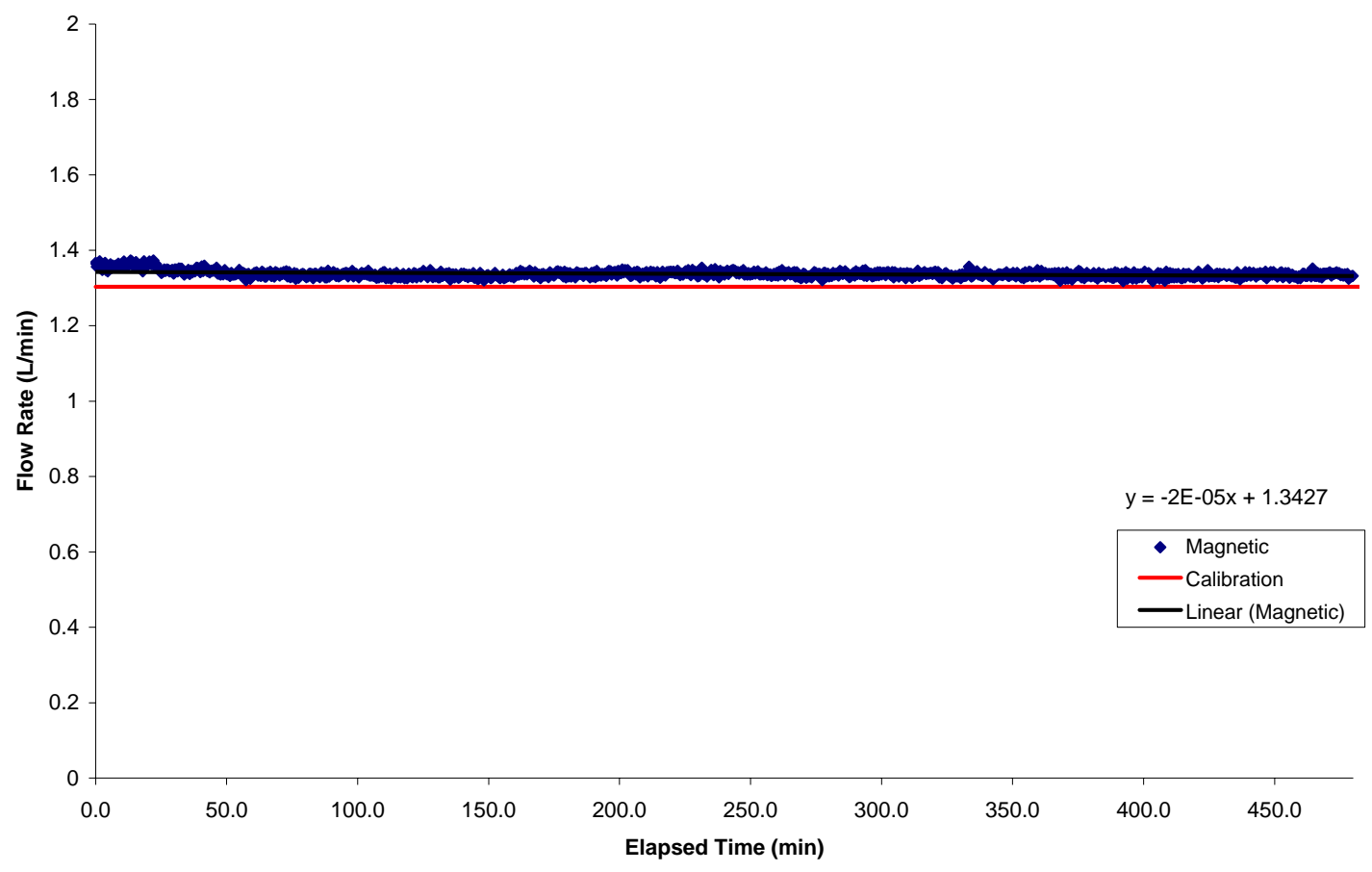

Fig. B.7. Magnetic response to steady-state flow at $7.2 \mathrm{~Hz}$ for 8 -h continuous operation.

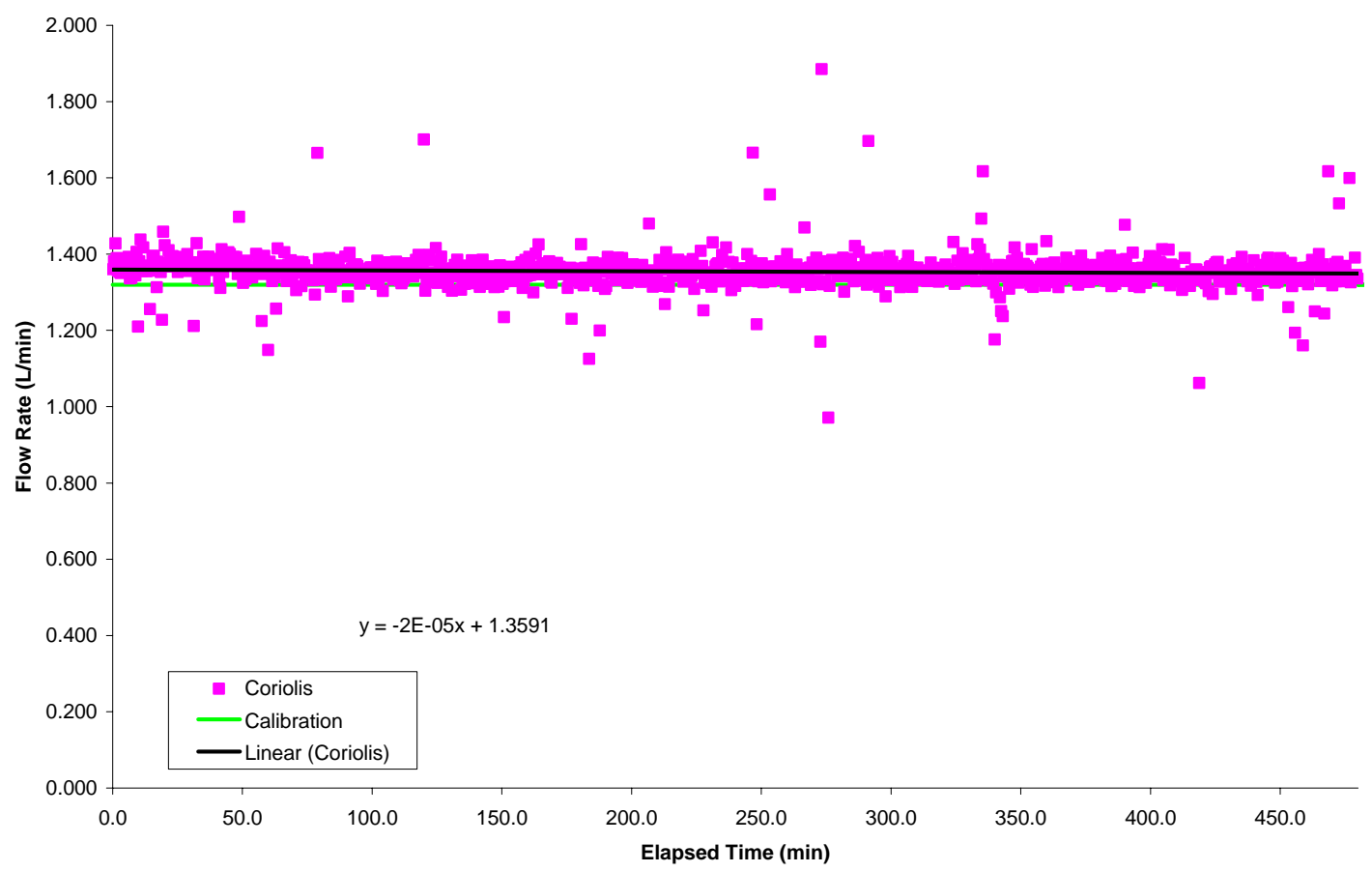

Fig. B.8. Coriolis response to steady-state flow at $7.2 \mathrm{~Hz}$ for 8 -h continuous operation. 


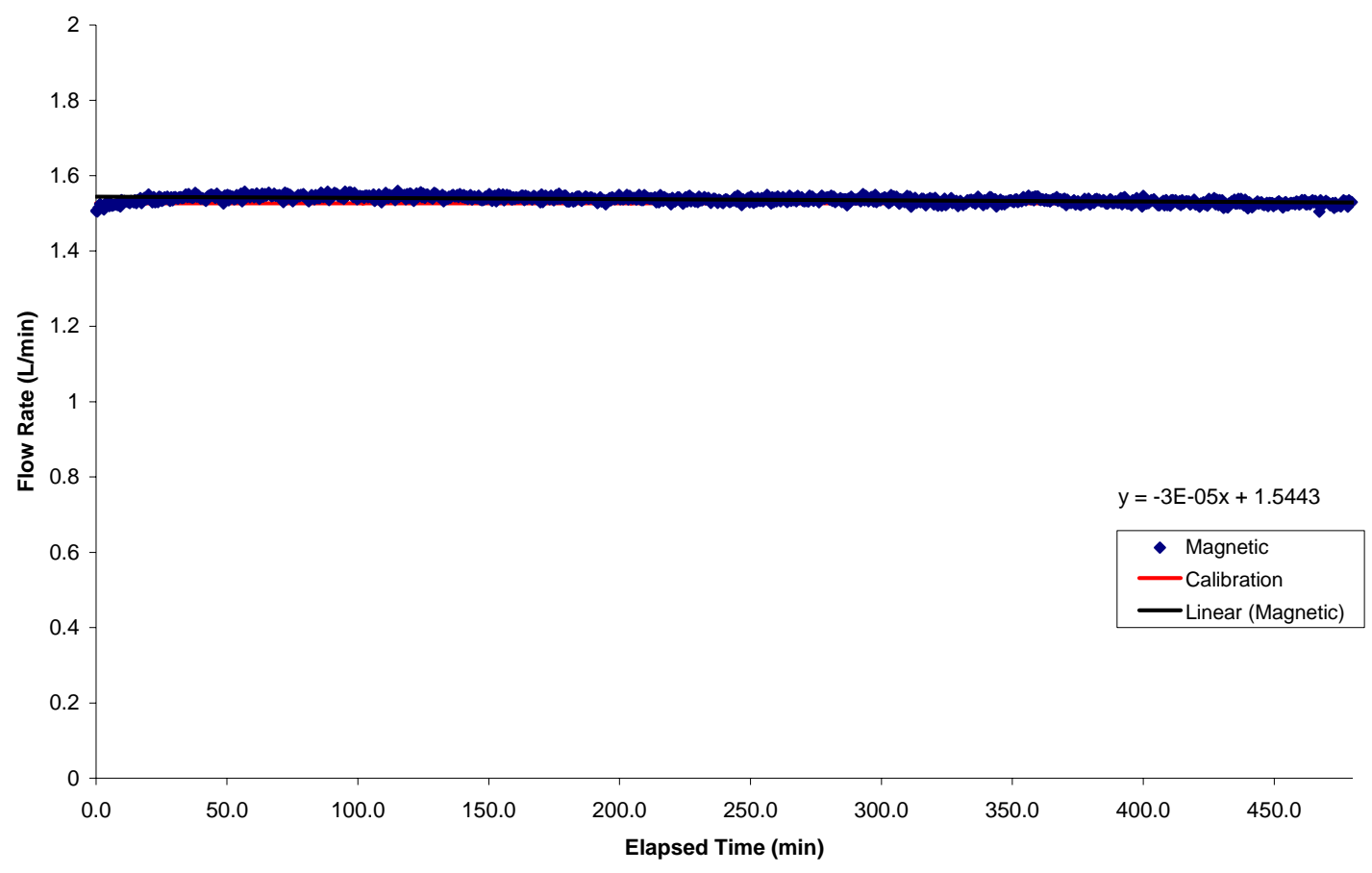

Fig. B.9. Magnetic response to steady-state flow at $8.3 \mathrm{~Hz}$ for 8 -h continuous operation.

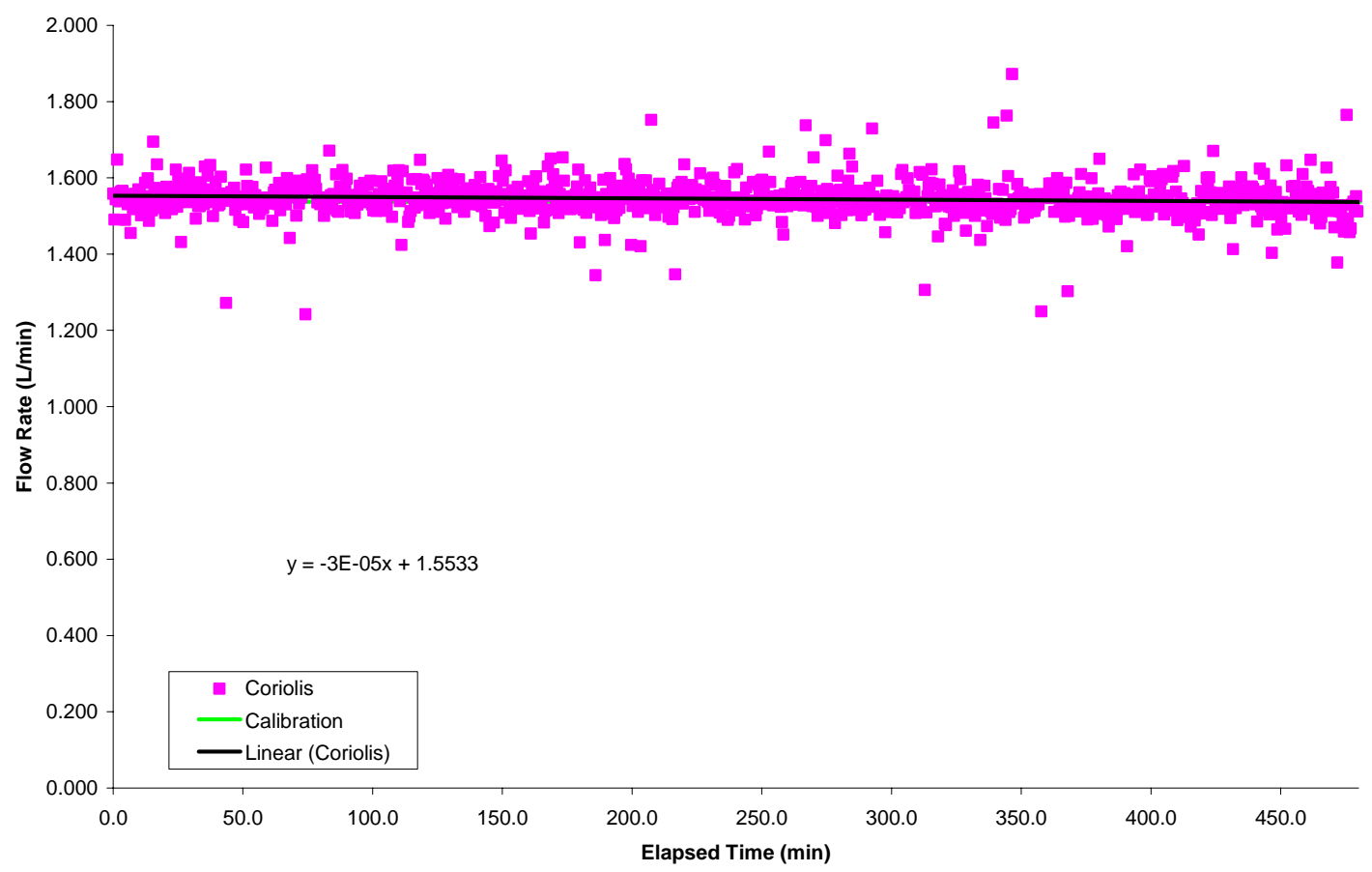

Fig. B.10. Coriolis response to steady-state flow at $8.3 \mathrm{~Hz}$ for 8 -h continuous operation. 


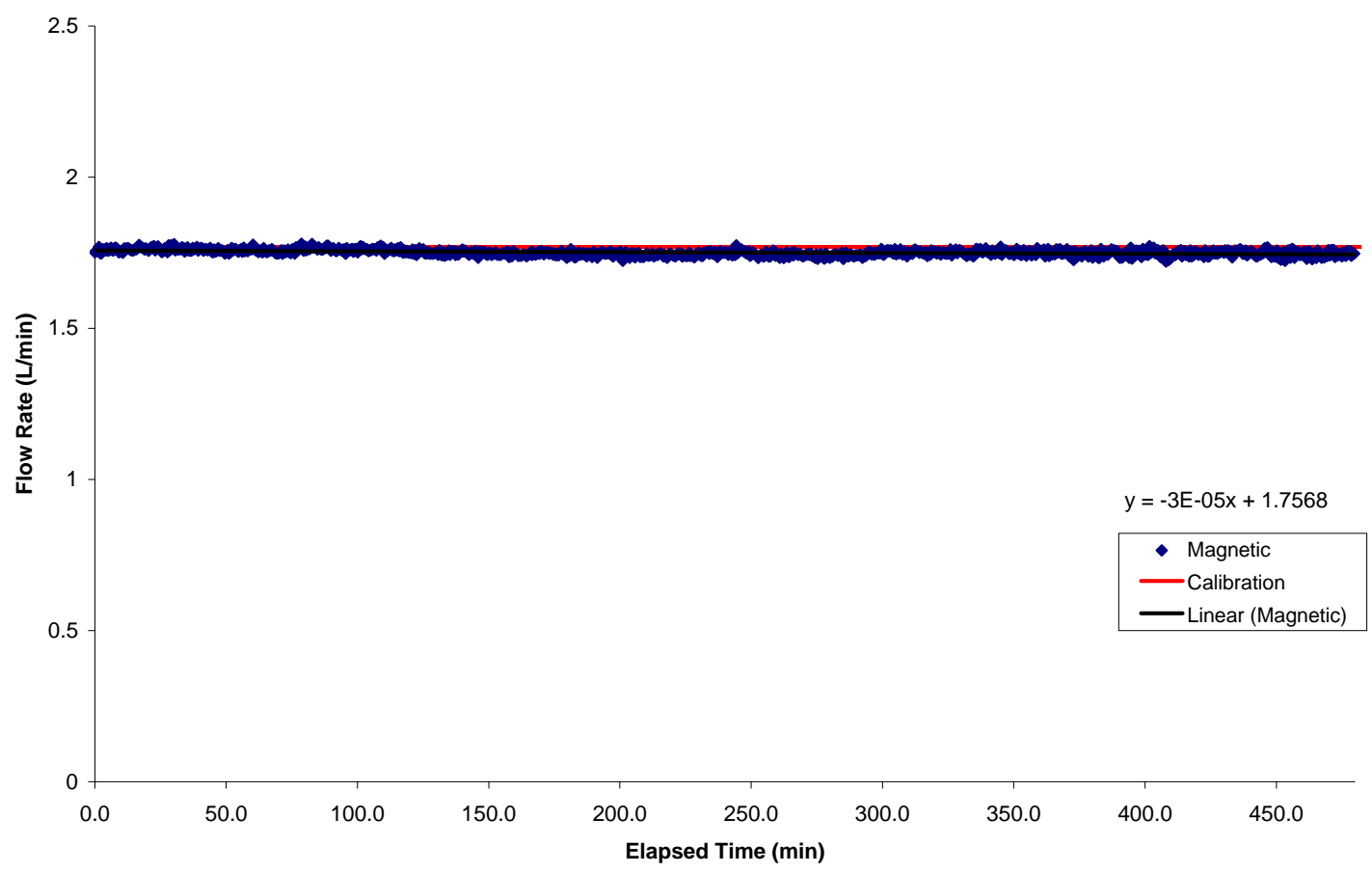

Fig. B.11. Magnetic response to steady-state flow at $9.5 \mathrm{~Hz}$ for 8 -h continuous operation.

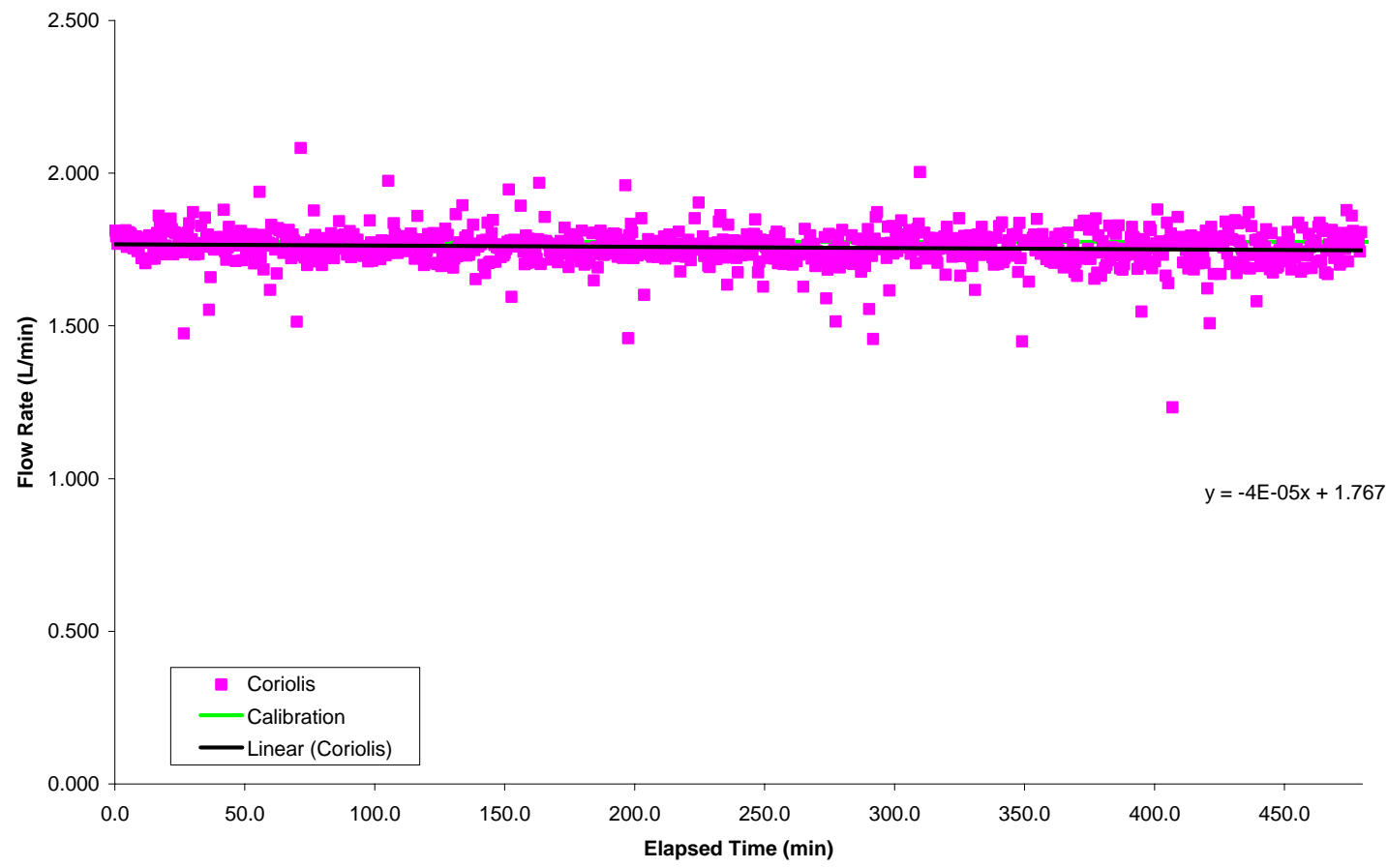

Fig. B.12. Coriolis response to steady-state flow at $9.5 \mathrm{~Hz}$ for 8 -h continuous operation. 


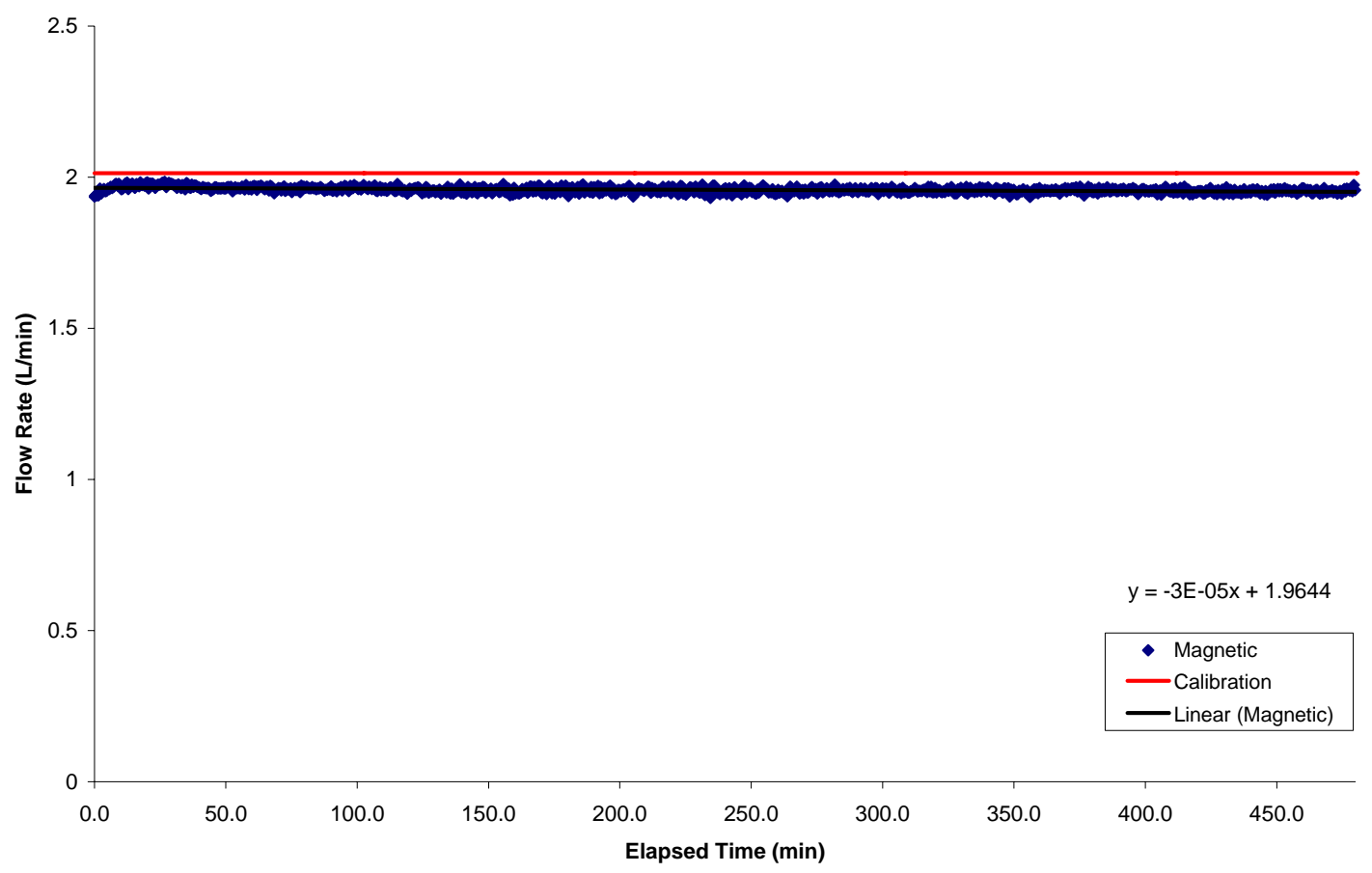

Fig. B.13. Magnetic response to steady-state flow at $10.7 \mathrm{~Hz}$ for 8-h continuous operation.

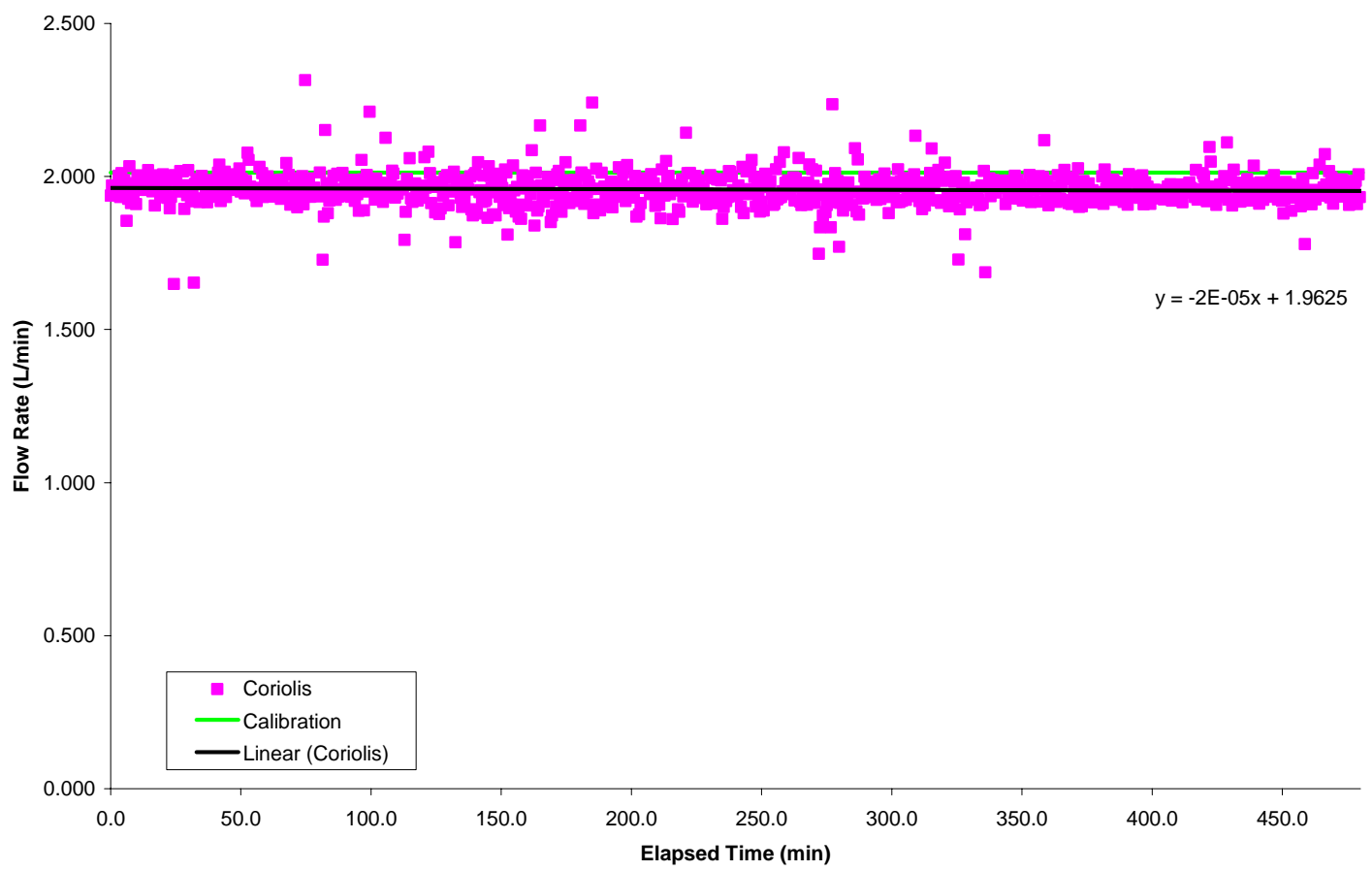

Fig. B.14. Coriolis response to steady-state flow at $10.7 \mathrm{~Hz}$ for 8 -h continuous operation. 


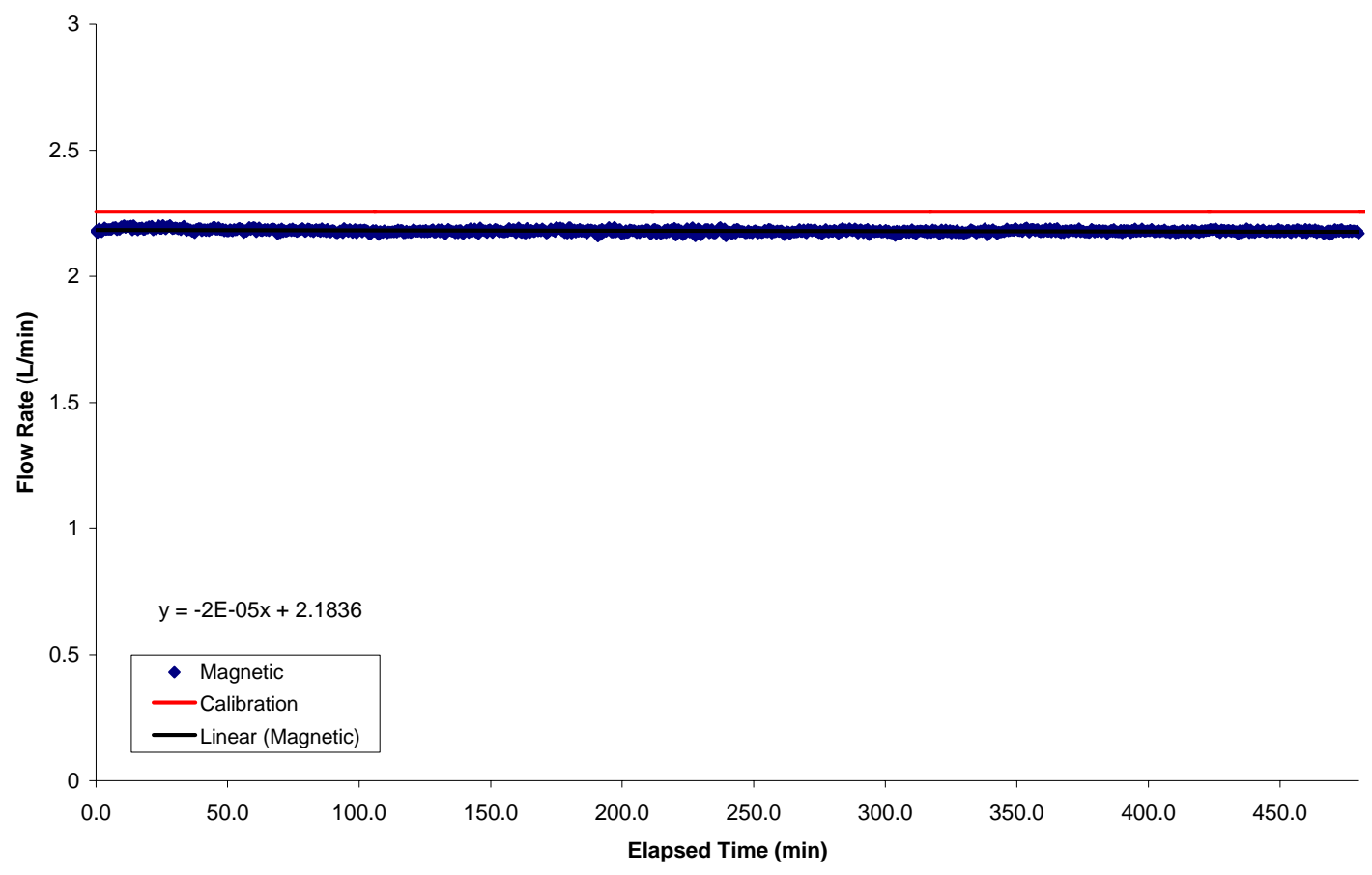

Fig. B.15. Magnetic response to steady-state flow at $11.9 \mathrm{~Hz}$ for 8 -h continuous operation.

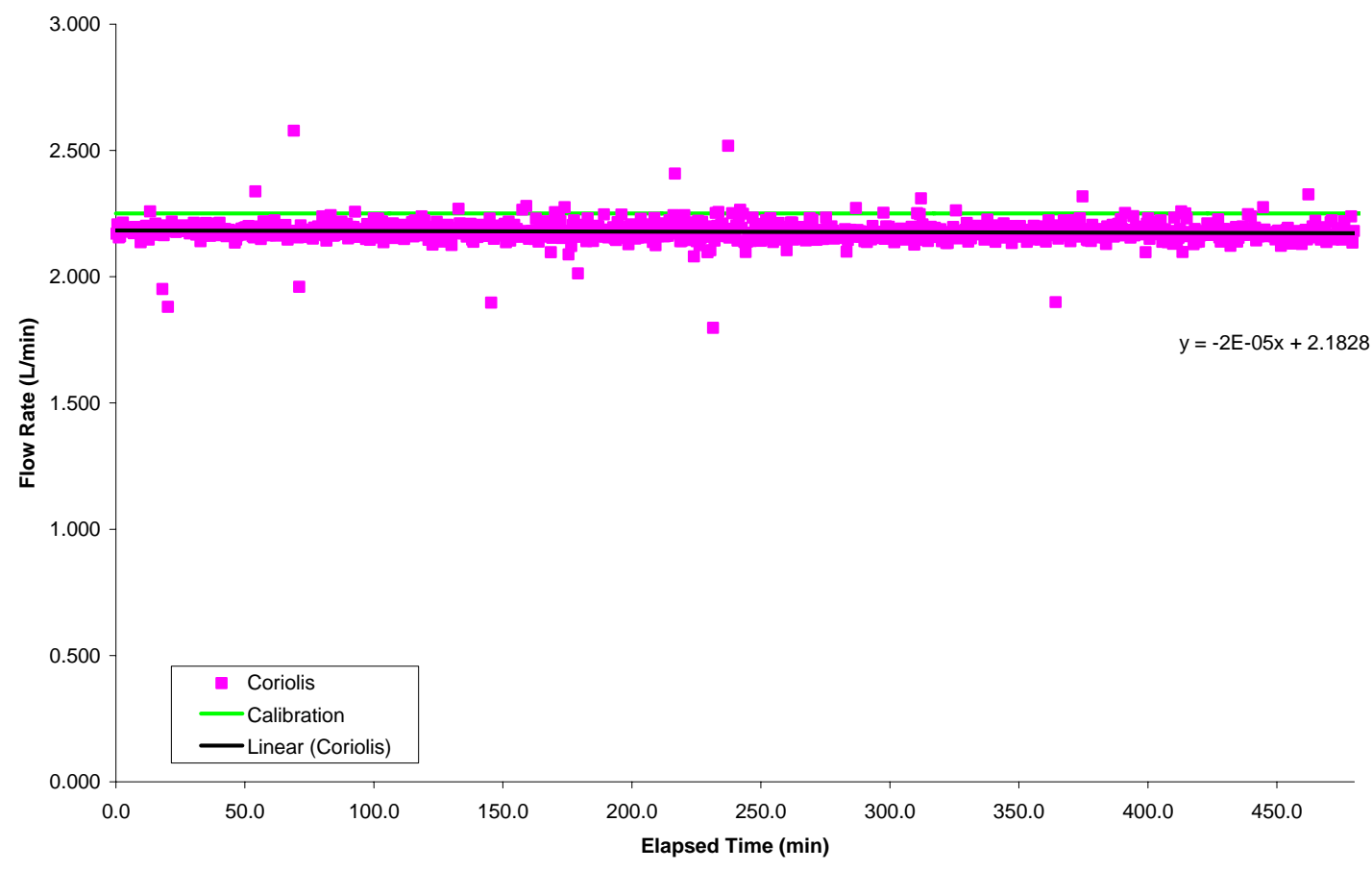

Fig. B.16. Coriolis response to steady-state flow at $11.9 \mathrm{~Hz}$ for 8 -h continuous operation. 
APPENDIX C

GRAPHS OF ENTRAINED AIR RESPONSE 



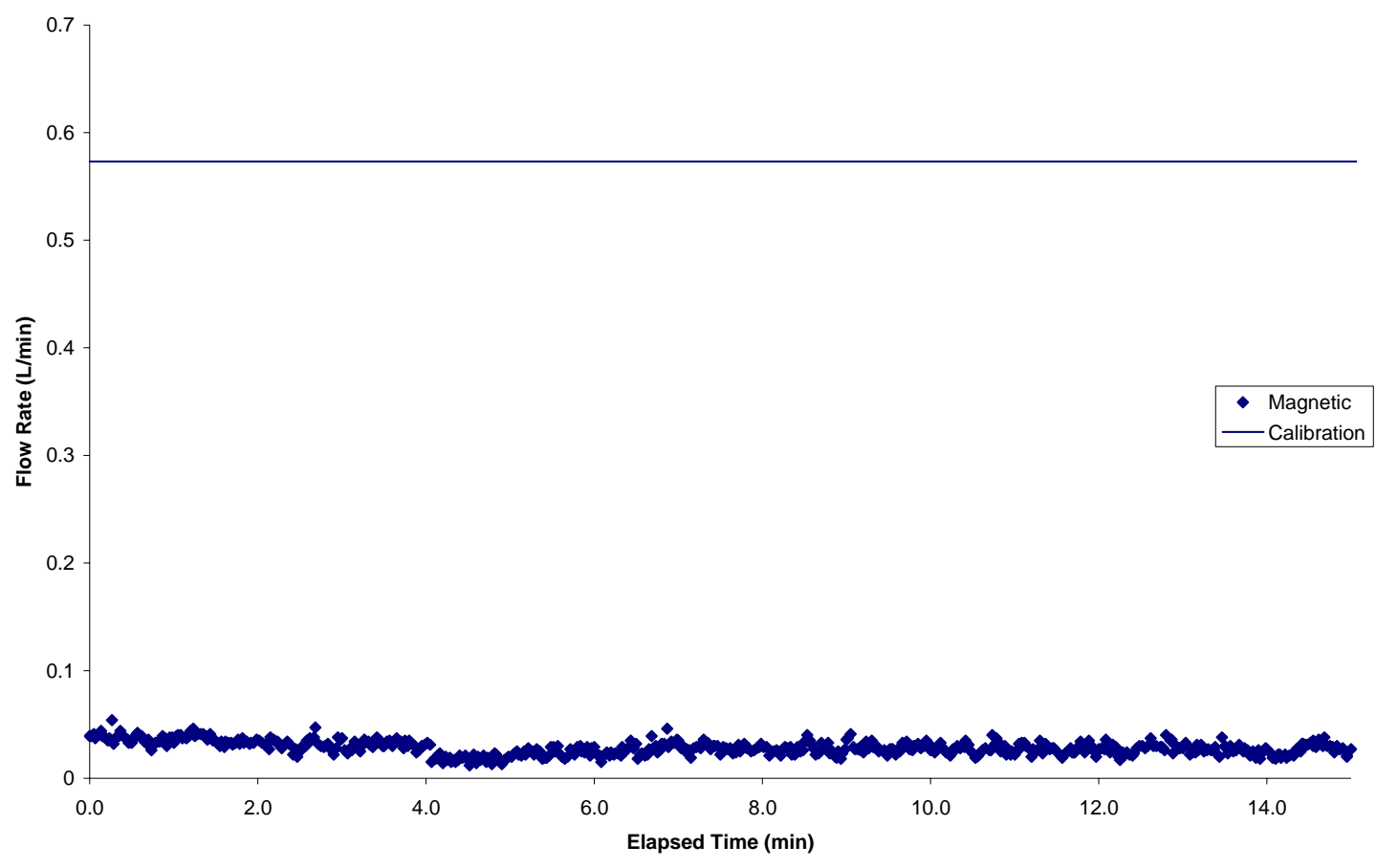

Fig. C.1. Magnetic response to entrained air (500 sccm) and pump speed of $3.6 \mathrm{~Hz}$.

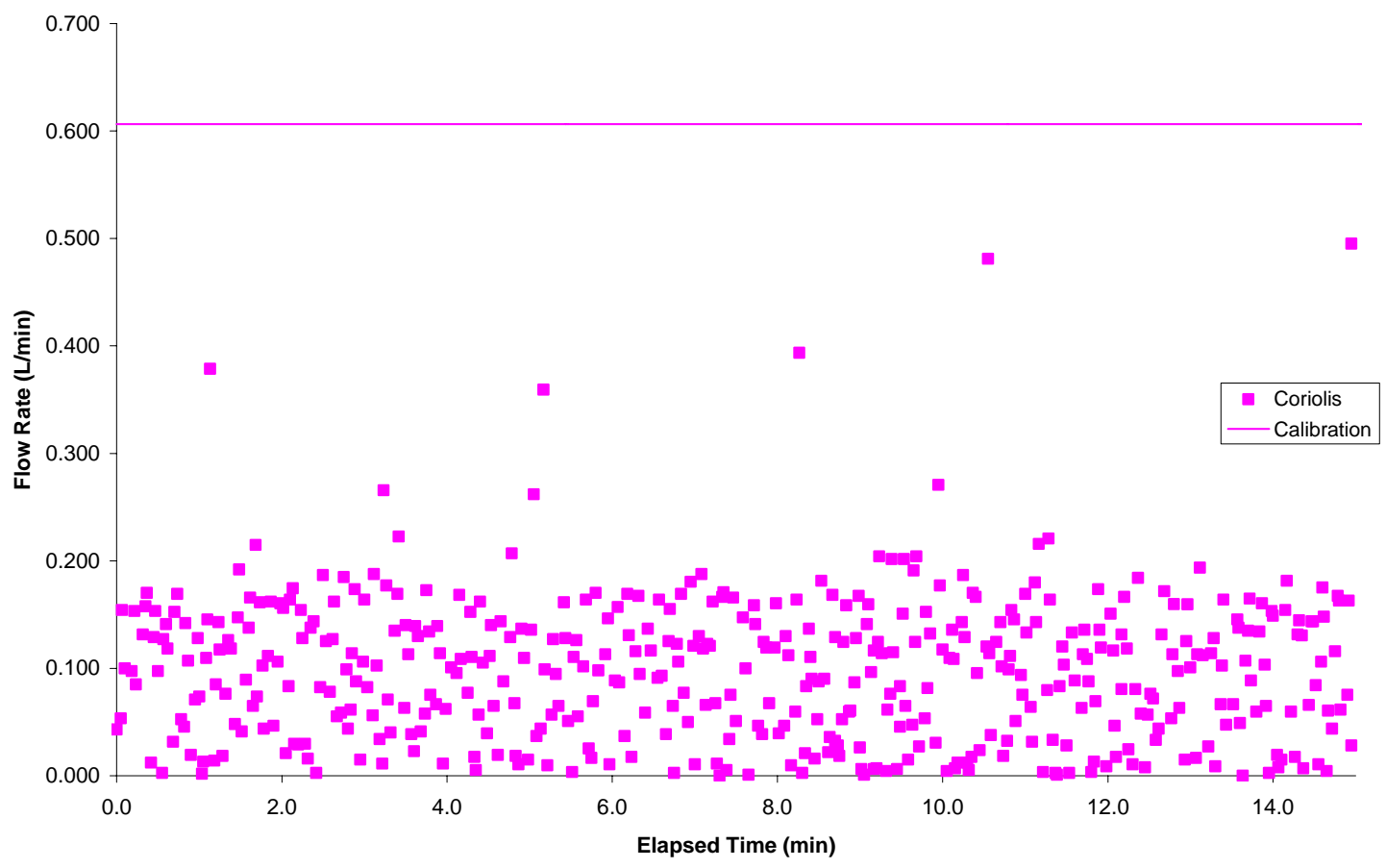

Fig. C.2. Coriolis response to entrained air (500 sccm) and pump speed of $3.6 \mathrm{~Hz}$. 


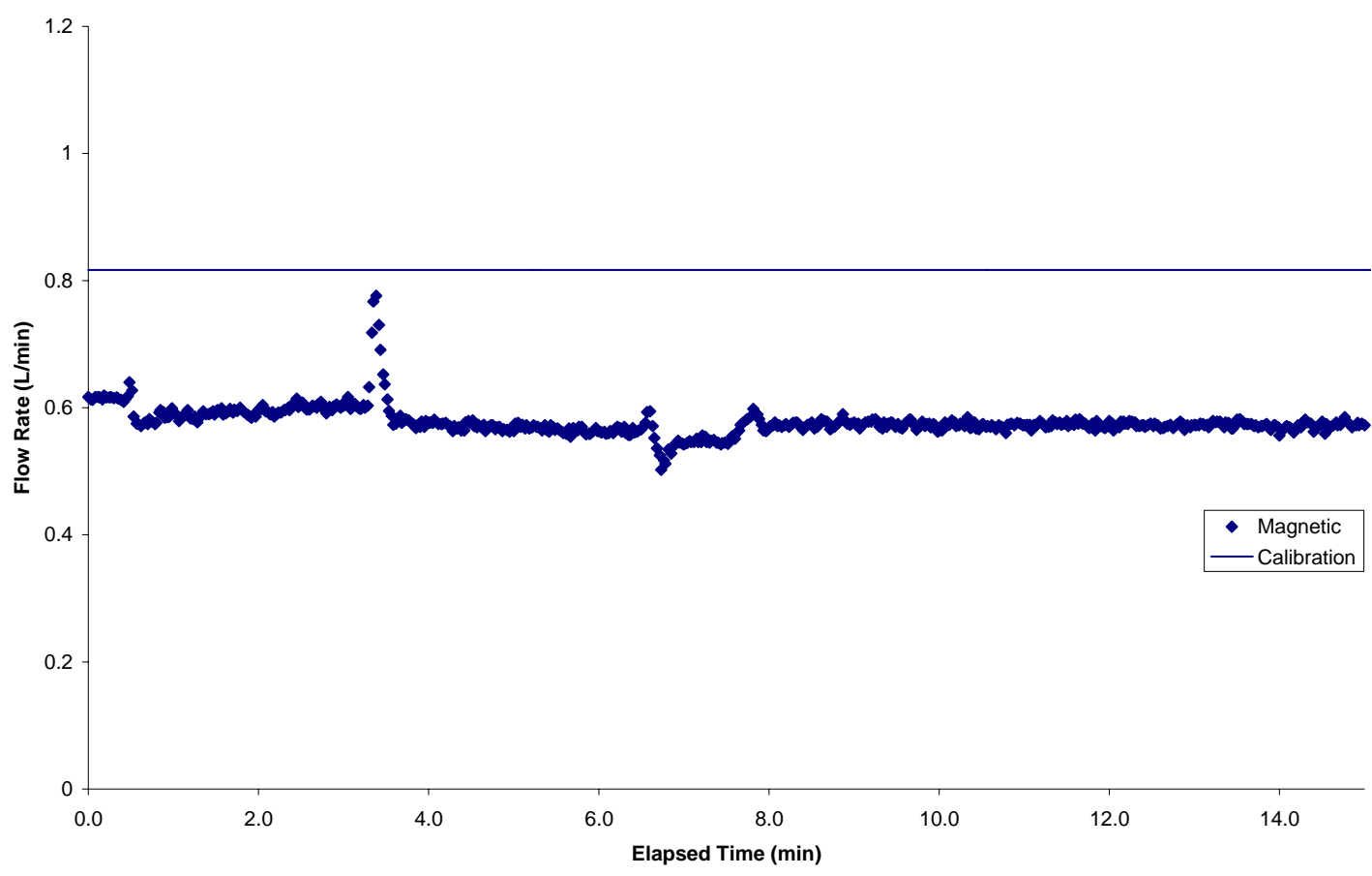

Fig. C.3. Magnetic response to entrained air (500 sccm) and pump speed of $4.8 \mathrm{~Hz}$.

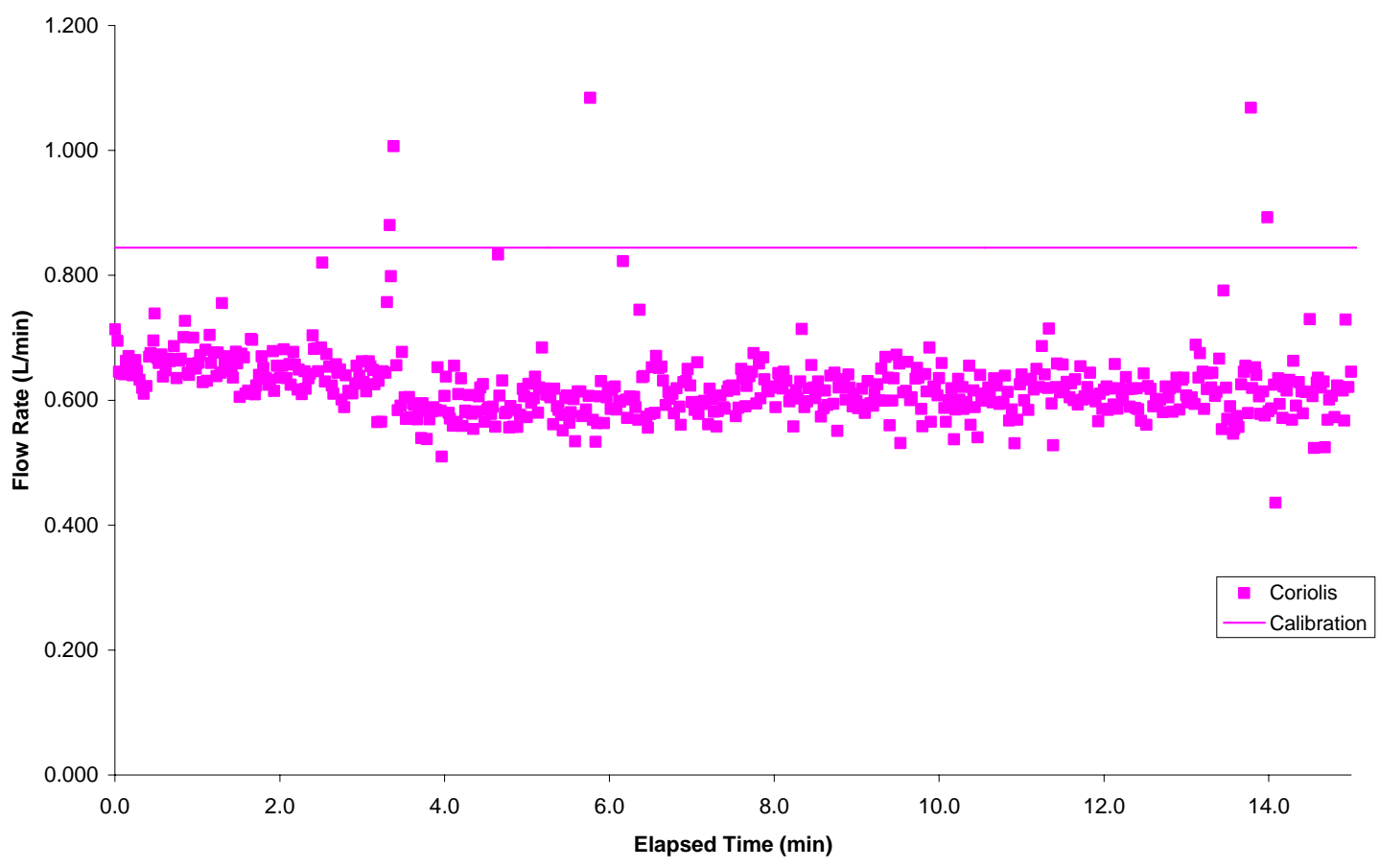

Fig. C.4. Coriolis response to entrained air $(500 \mathrm{sccm})$ and pump speed of $4.8 \mathrm{~Hz}$. 


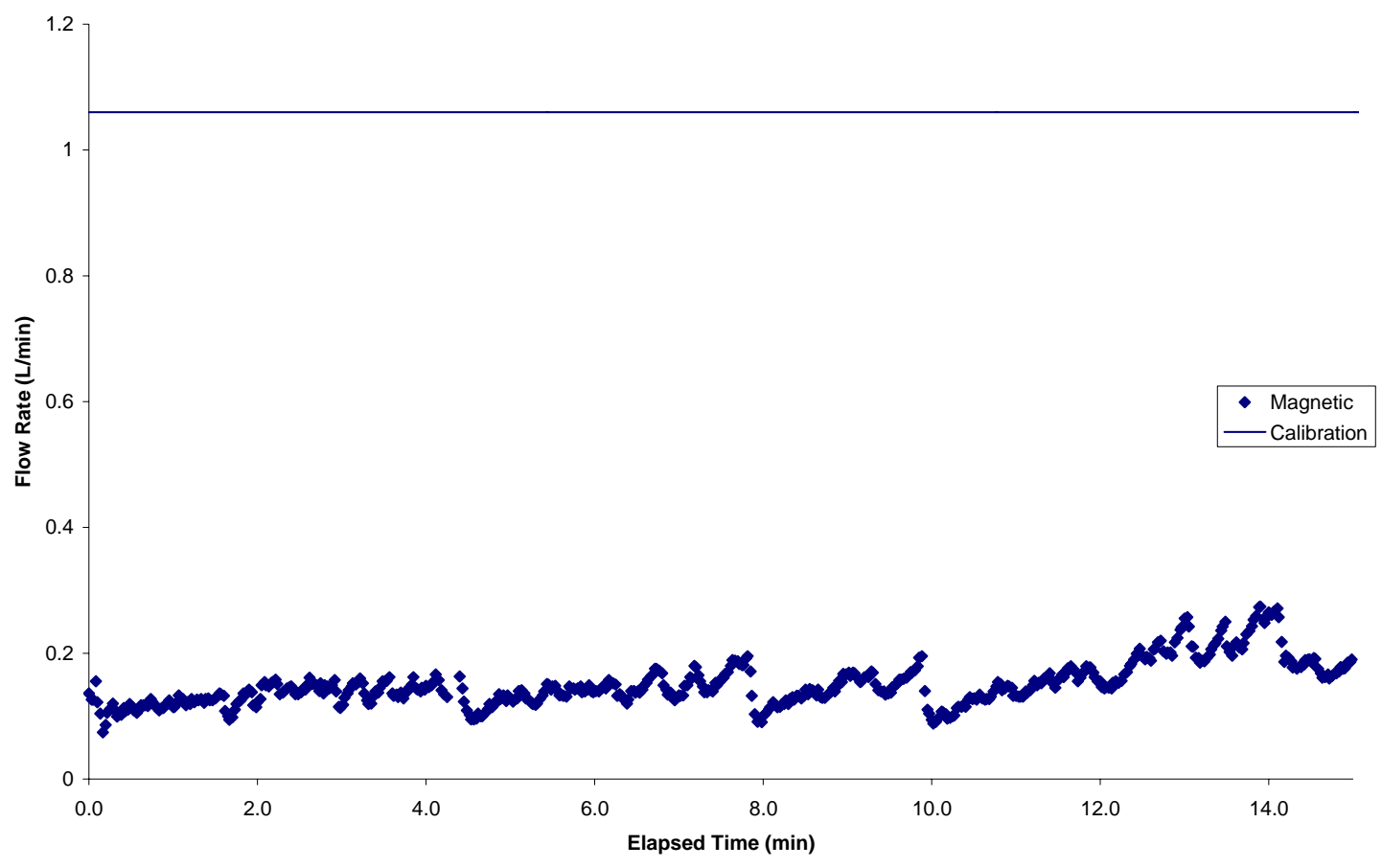

Fig. C.5. Magnetic response to entrained air (500 sccm) and pump speed of $6.0 \mathrm{~Hz}$.

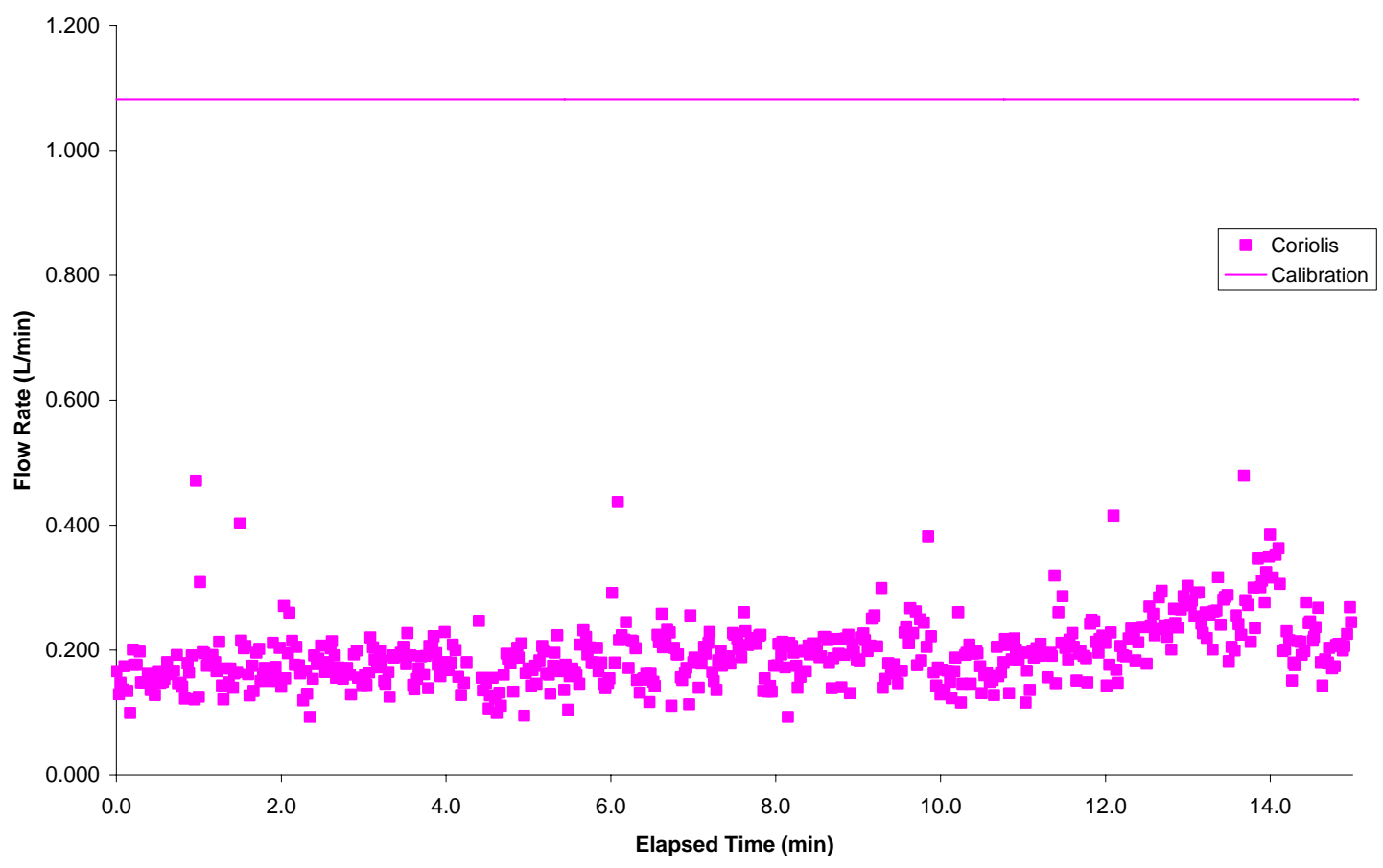

Fig. C.6. Coriolis response to entrained air (500 sccm) and pump speed of $6.0 \mathrm{~Hz}$. 


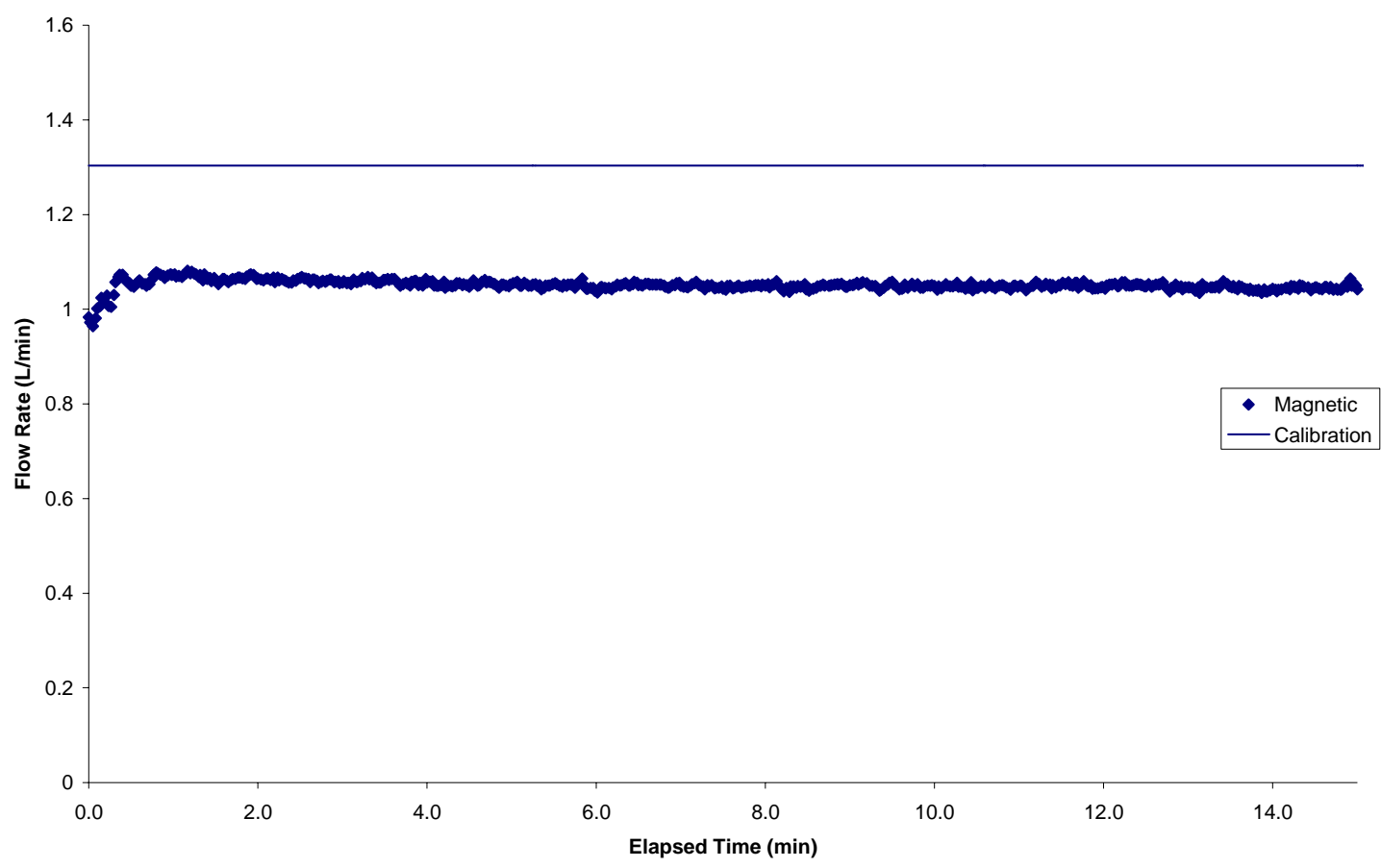

Fig. C.7. Magnetic response to entrained air (500 sccm) and pump speed of 7.2 Hz.

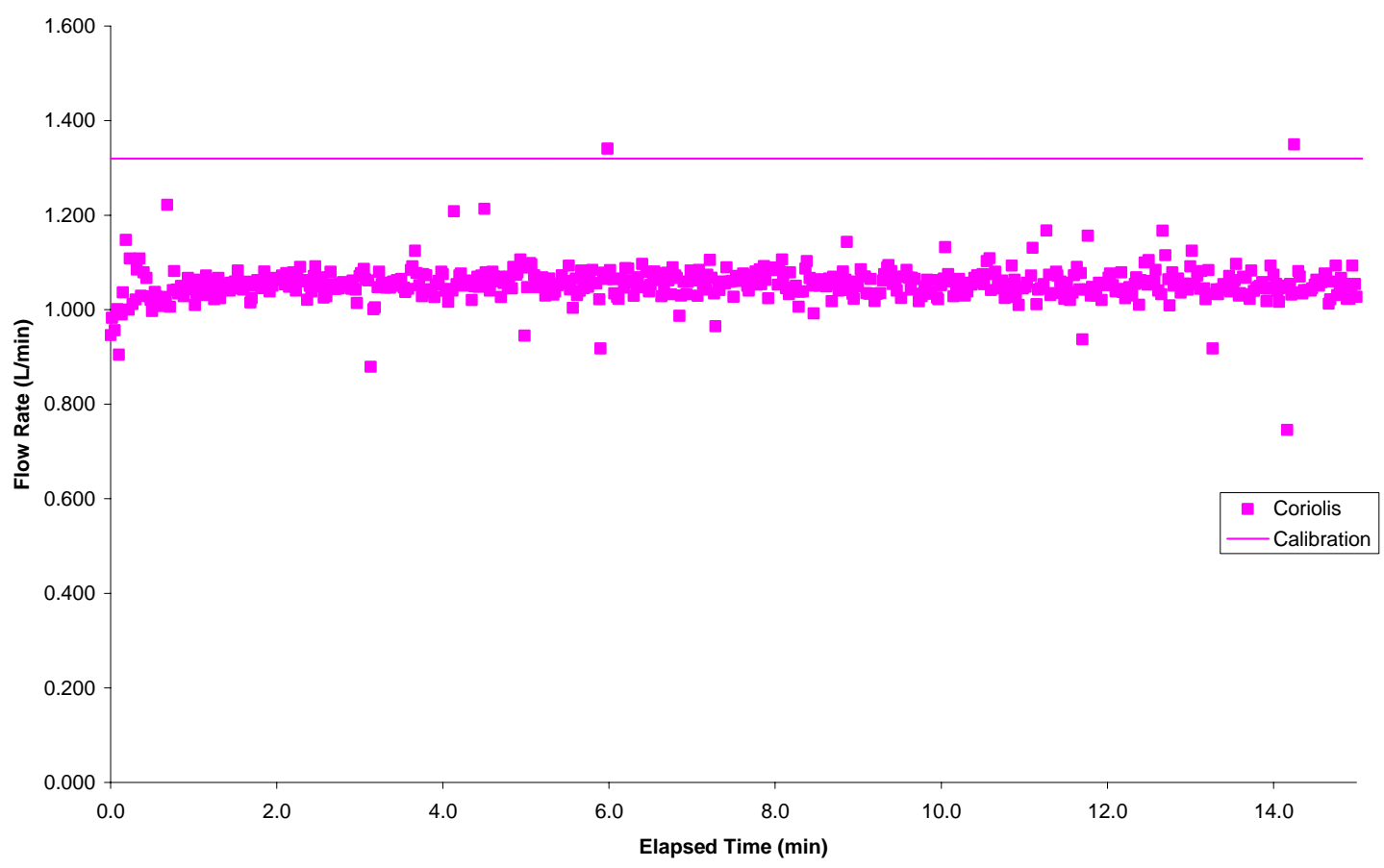

Fig. C.8. Coriolis response to entrained air (500 sccm) and pump speed of $7.2 \mathrm{~Hz}$. 


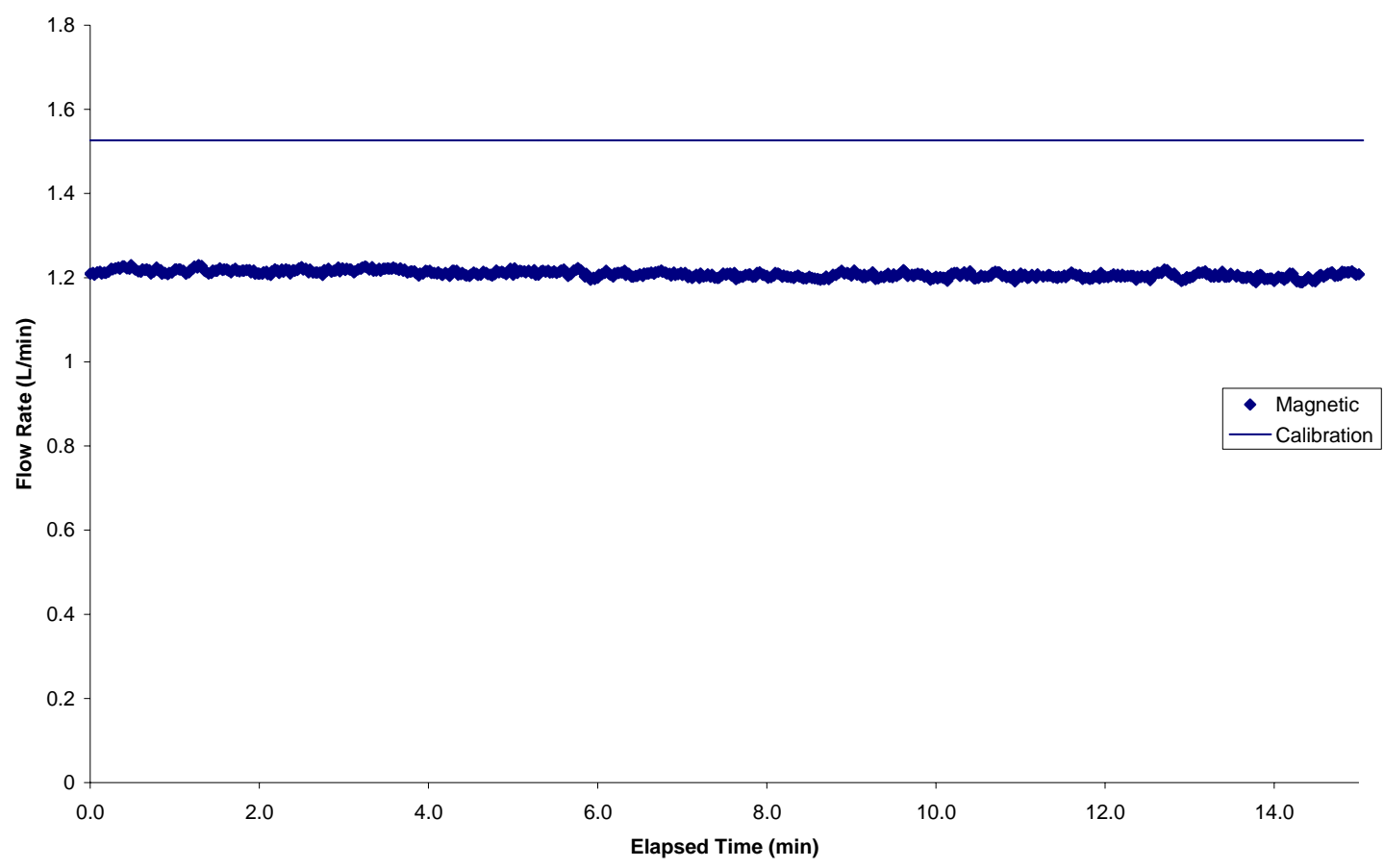

Fig. C.9. Magnetic response to entrained air (500 sccm) and pump speed of $8.3 \mathrm{~Hz}$.

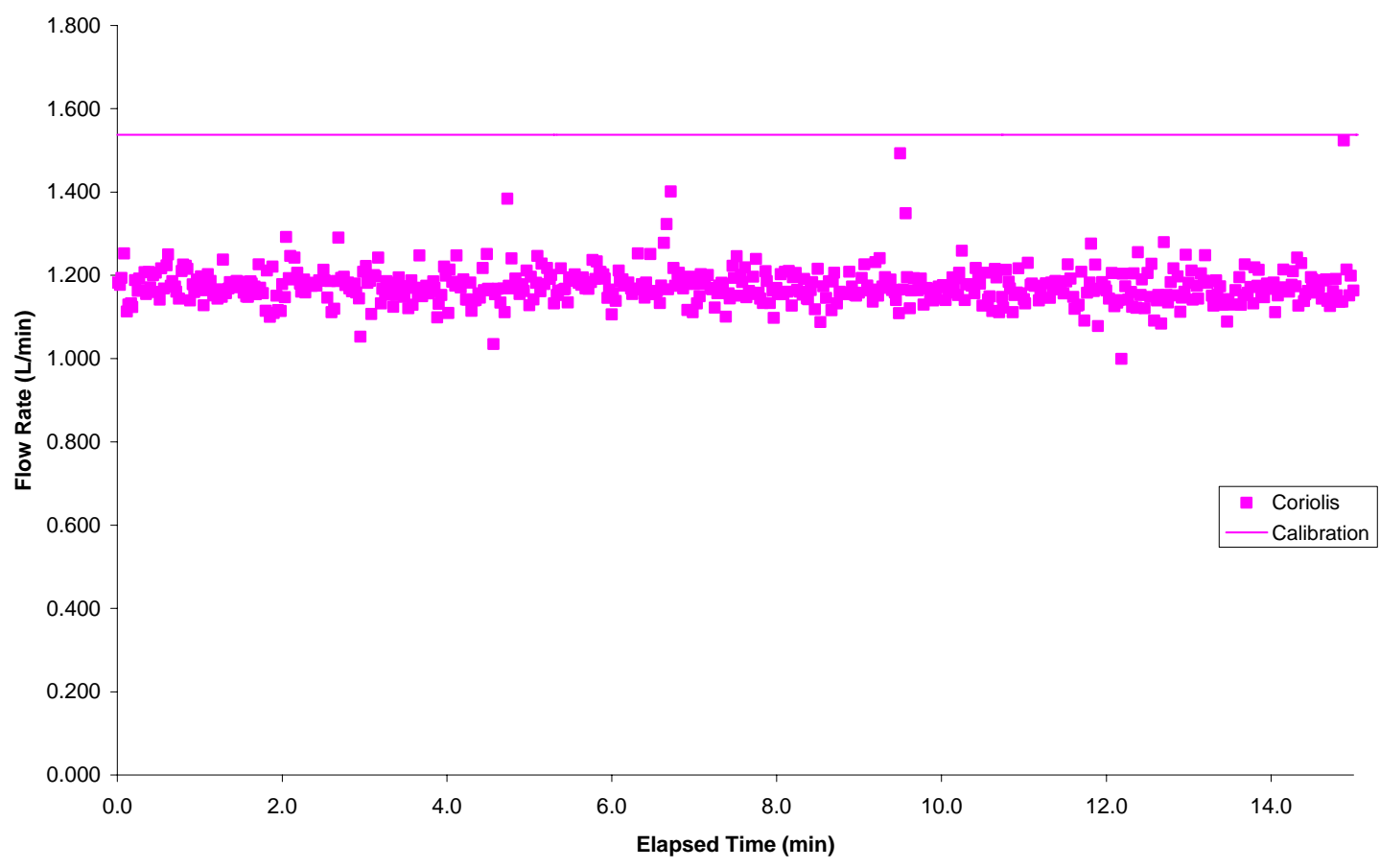

Fig. C.10. Coriolis response to entrained air $(500 \mathrm{sccm})$ and pump speed of $8.3 \mathrm{~Hz}$. 


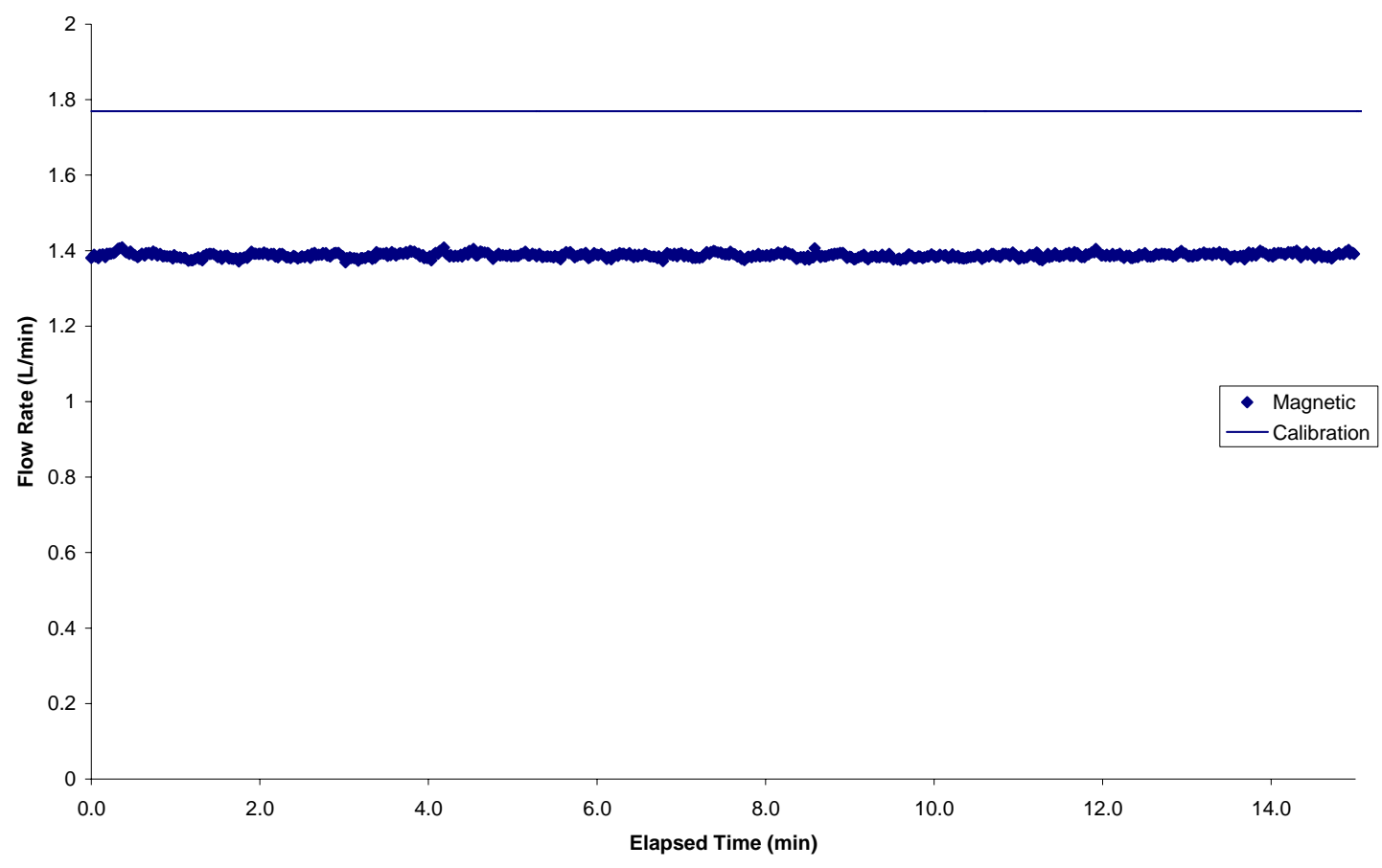

Fig. C.11. Magnetic response to entrained air $(500 \mathrm{sccm})$ and pump speed of $9.5 \mathrm{~Hz}$.

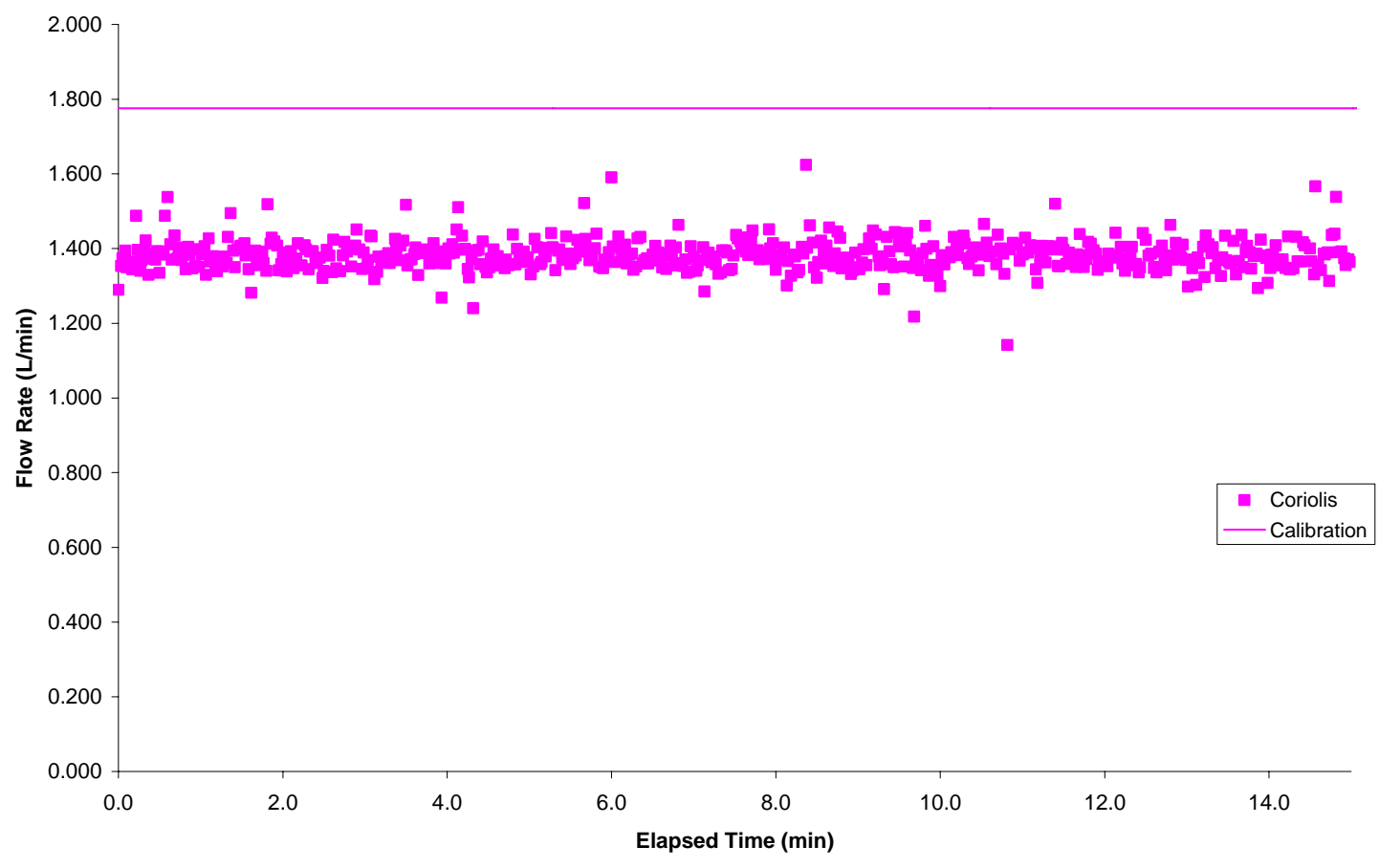

Fig. C.12. Coriolis response to entrained air (500 sccm) and pump speed of $9.5 \mathrm{~Hz}$. 


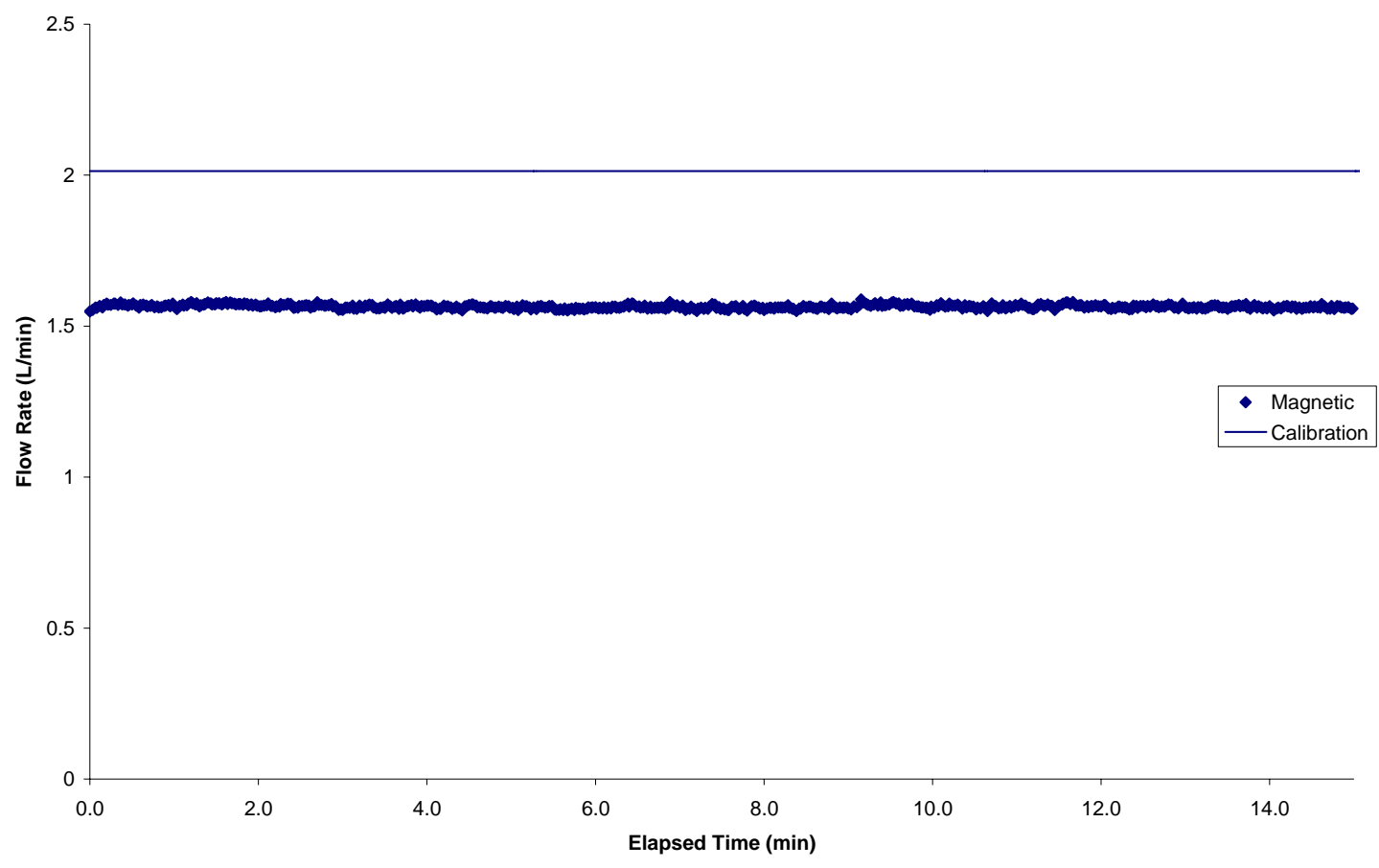

Fig. C.13. Magnetic response to entrained air (500 sccm) and pump speed of $10.7 \mathrm{~Hz}$.

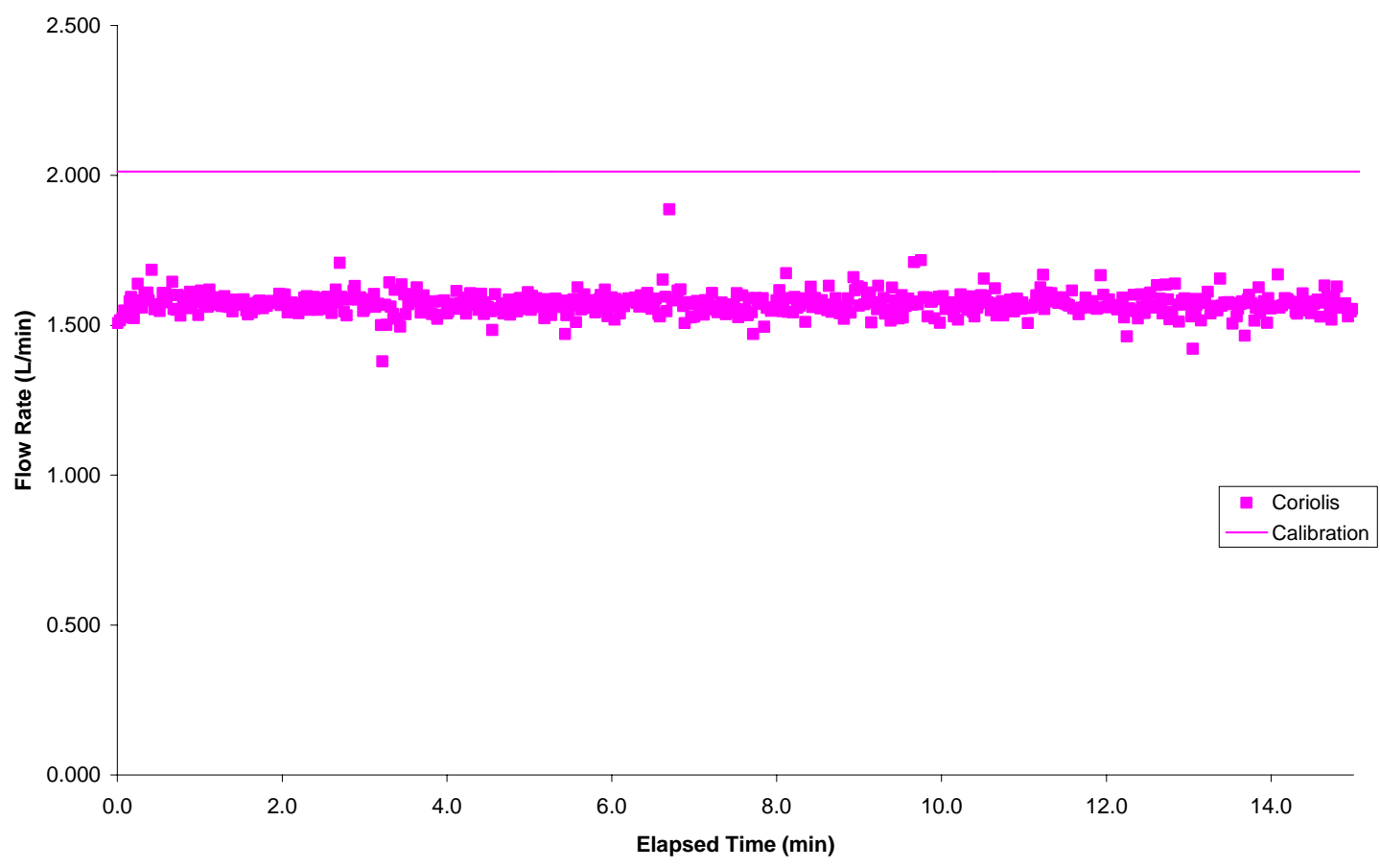

Fig. C.14. Coriolis response to entrained air $(500 \mathrm{sccm})$ and pump speed of $10.7 \mathrm{~Hz}$. 


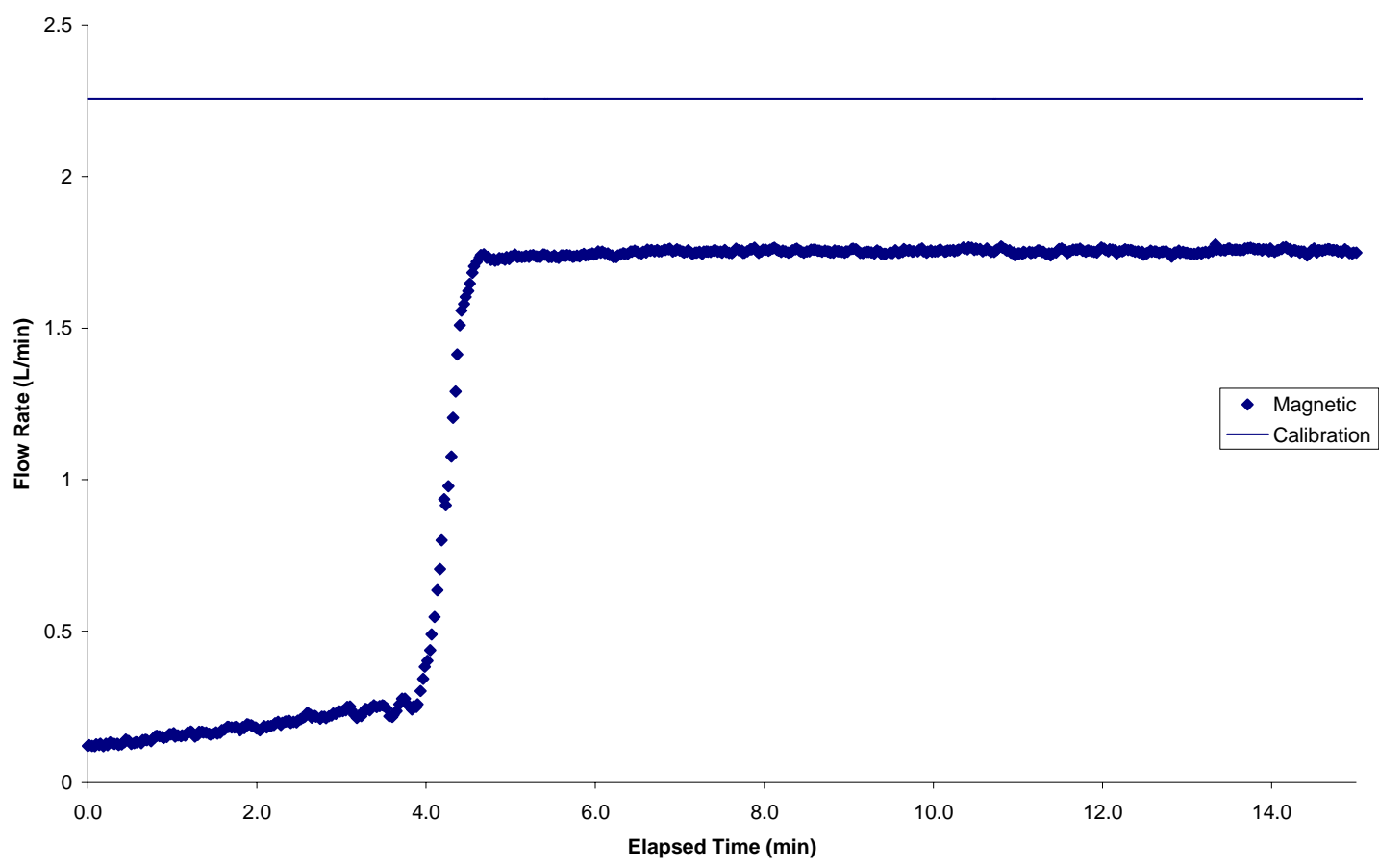

Fig. C.15. Magnetic response to entrained air (500 sccm) and pump speed of $11.9 \mathrm{~Hz}$.

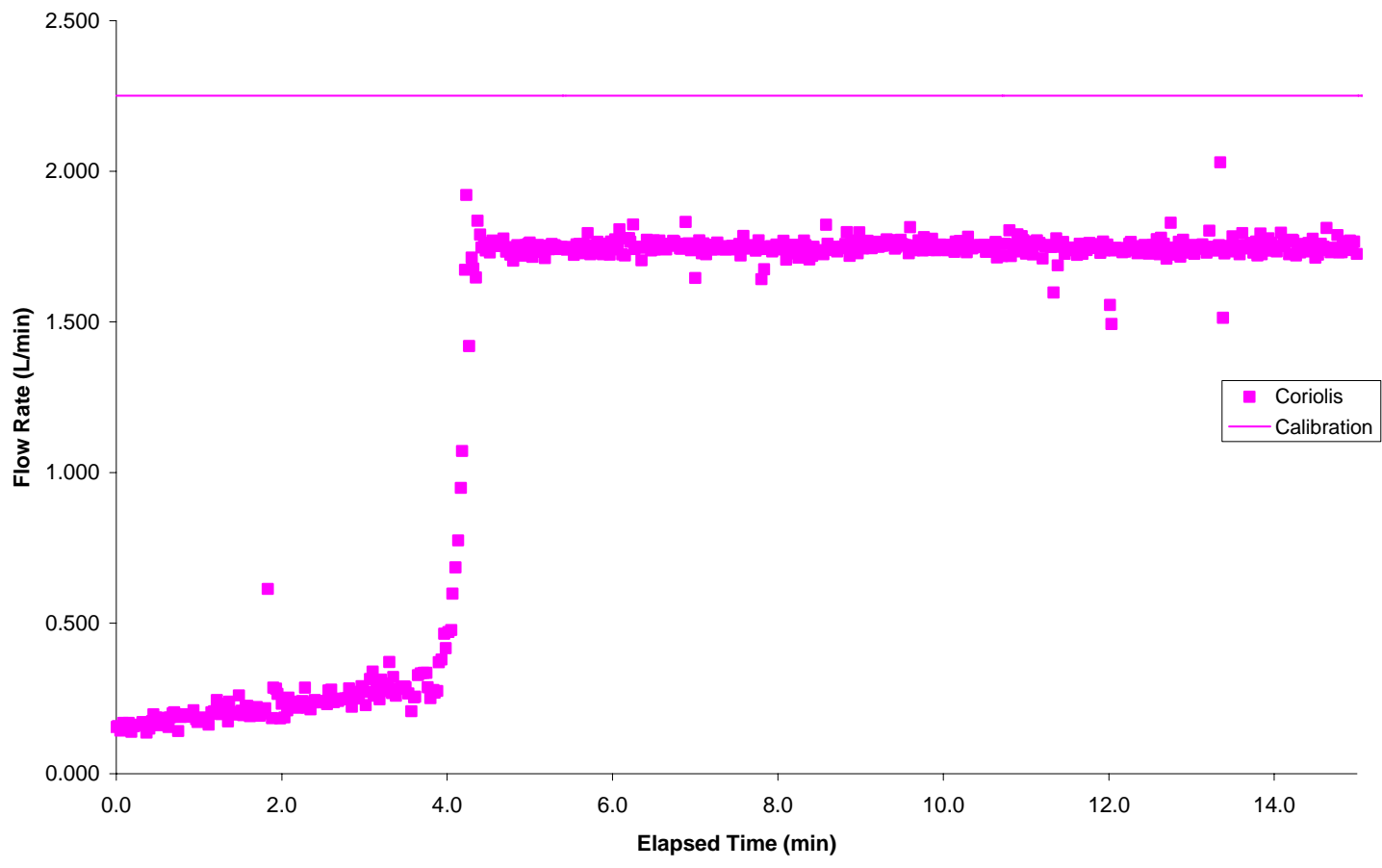

Fig. C.16. Coriolis response to entrained air $(500 \mathrm{sccm})$ and pump speed of $11.9 \mathrm{~Hz}$. 
APPENDIX D

GRAPHS OF PULSATING FLOW RESPONSE 



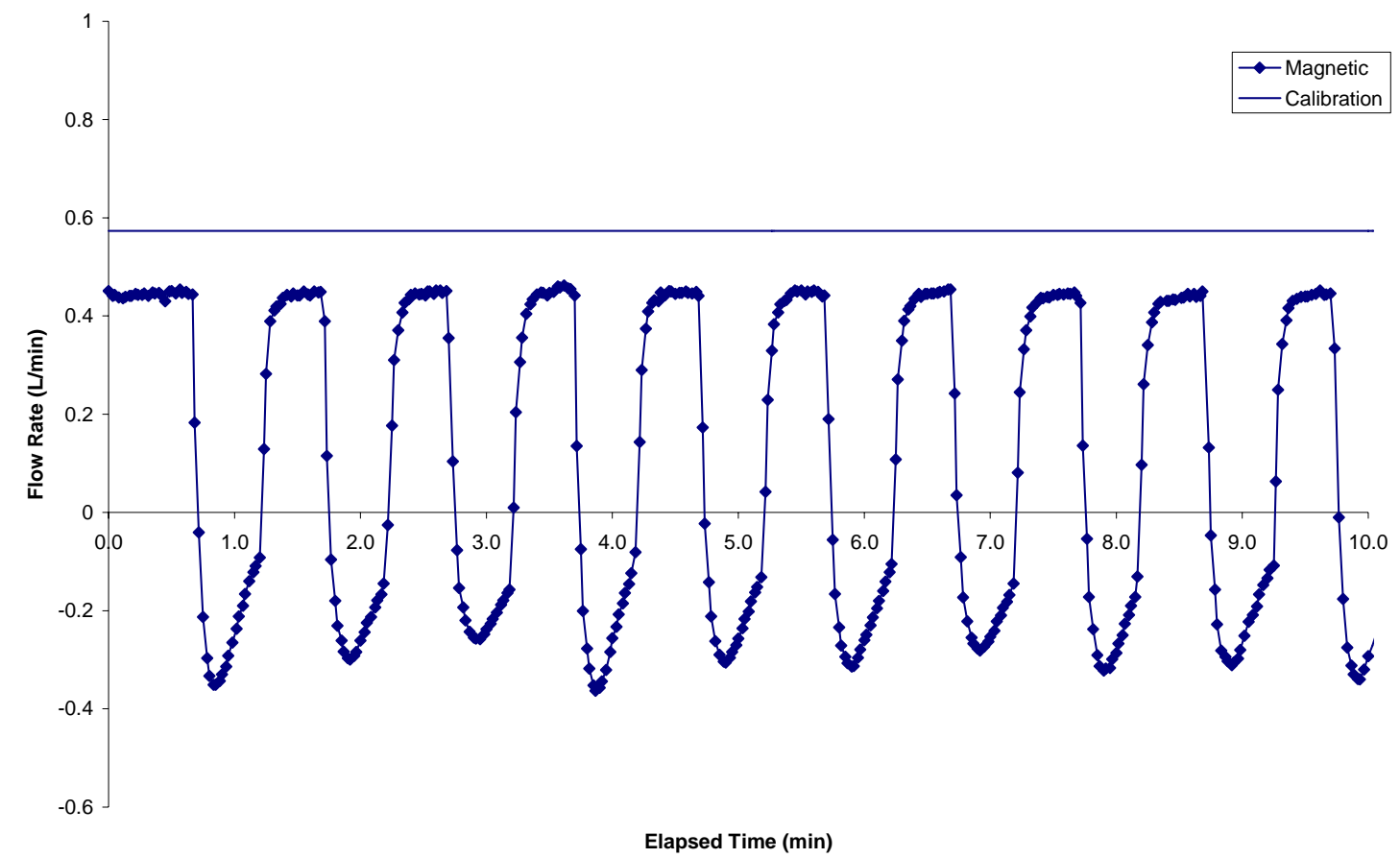

Fig. D.1. Magnetic response to pulsating flow at a pump speed of $3.6 \mathrm{~Hz}$.

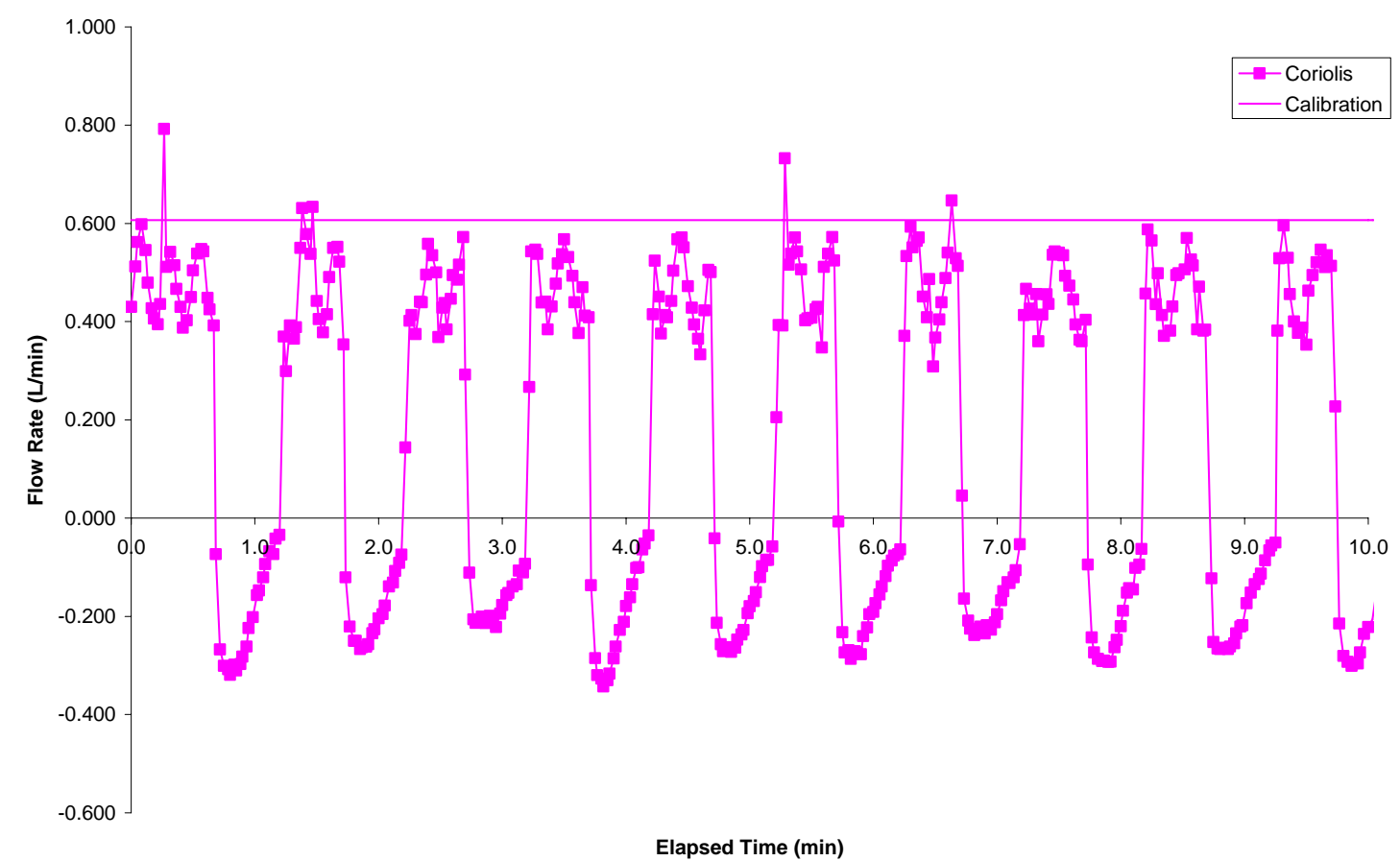

Fig. D.2. Coriolis response to pulsating flow at a pump speed of $3.6 \mathrm{~Hz}$.

D-3 


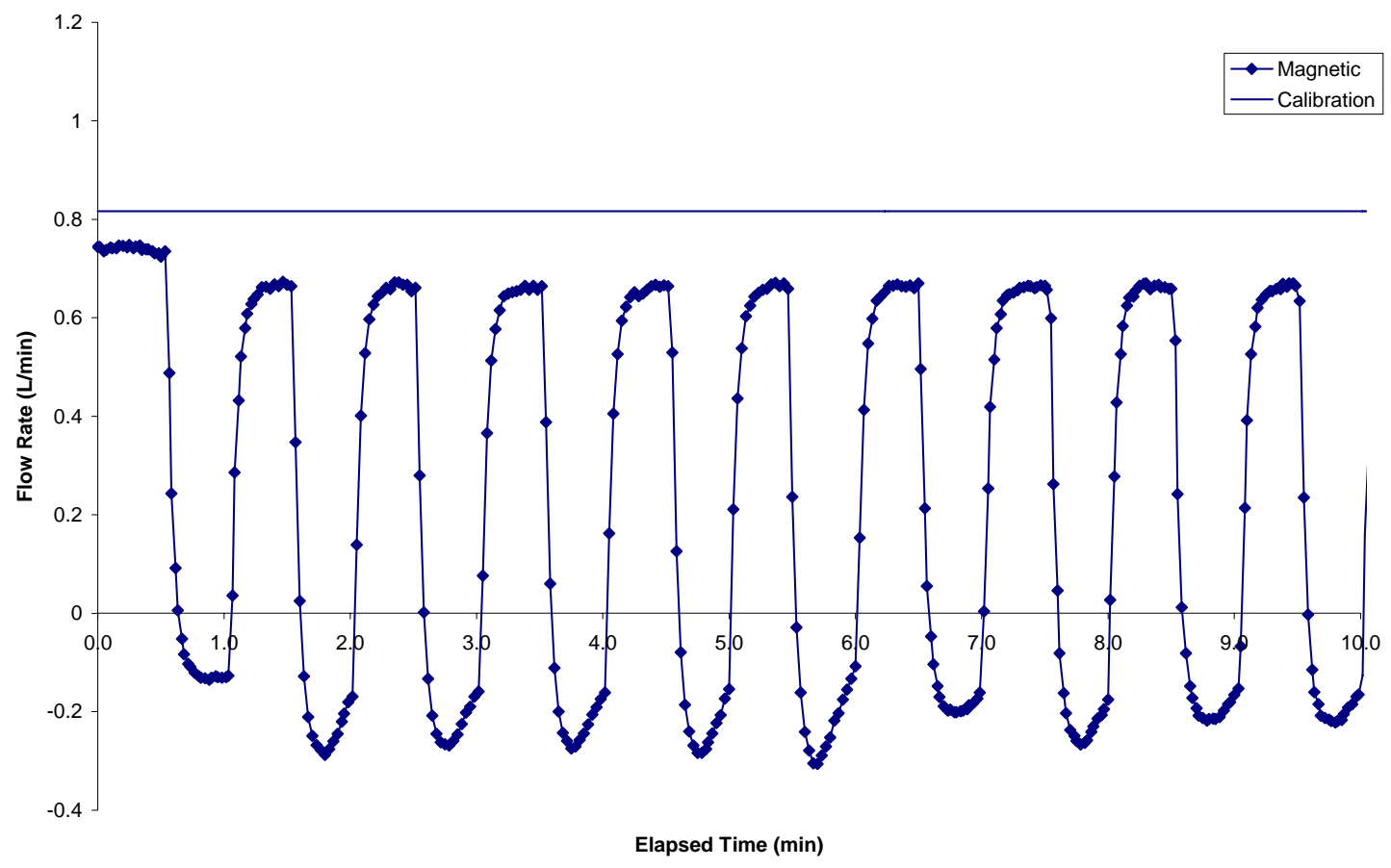

Fig. D.3. Magnetic response to pulsating flow at a pump speed of $4.8 \mathrm{~Hz}$.

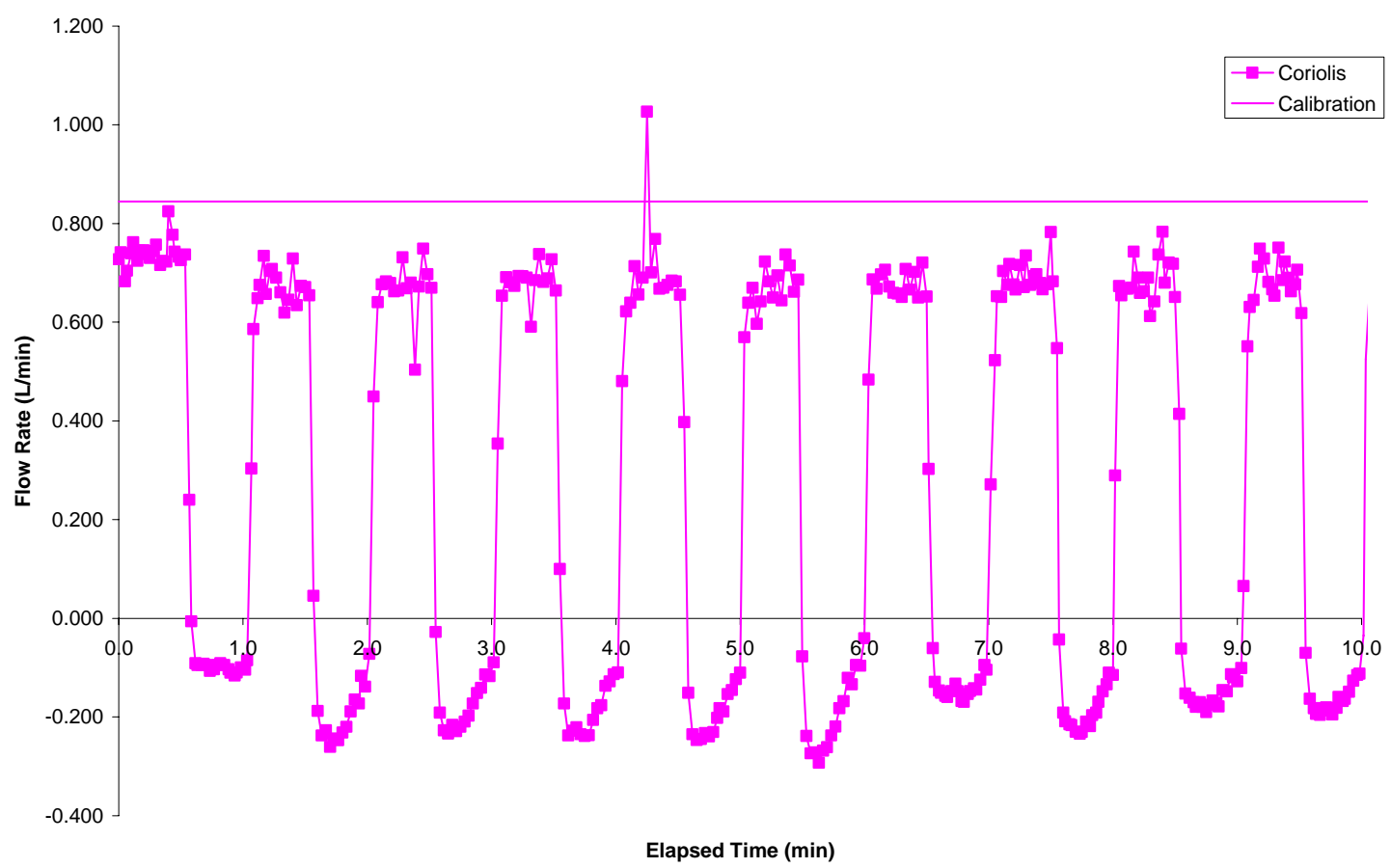

Fig. D.4. Coriolis response to pulsating flow at a pump speed of $4.8 \mathrm{~Hz}$. 


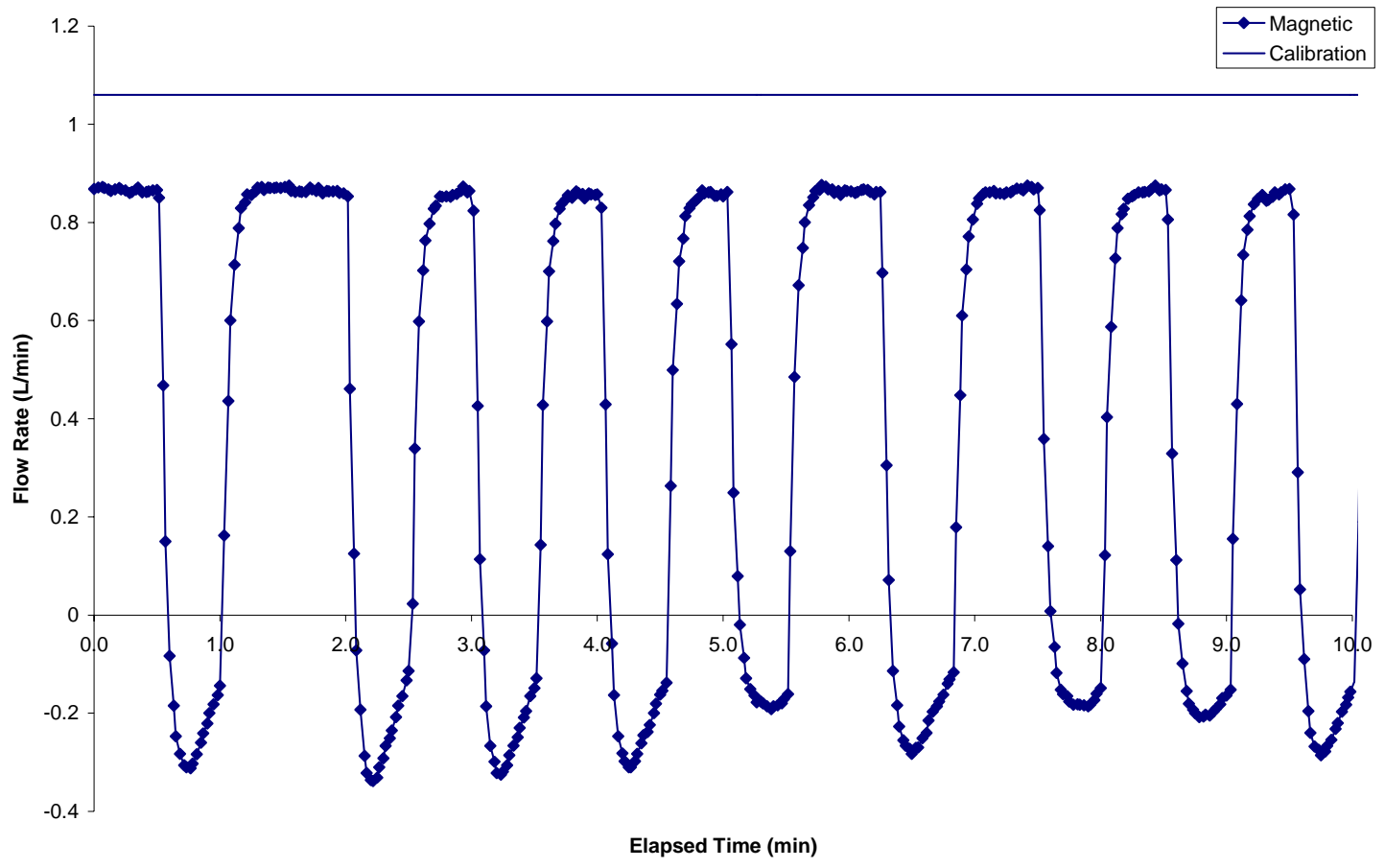

Fig. D.5. Magnetic response to pulsating flow at a pump speed of $6.0 \mathrm{~Hz}$.

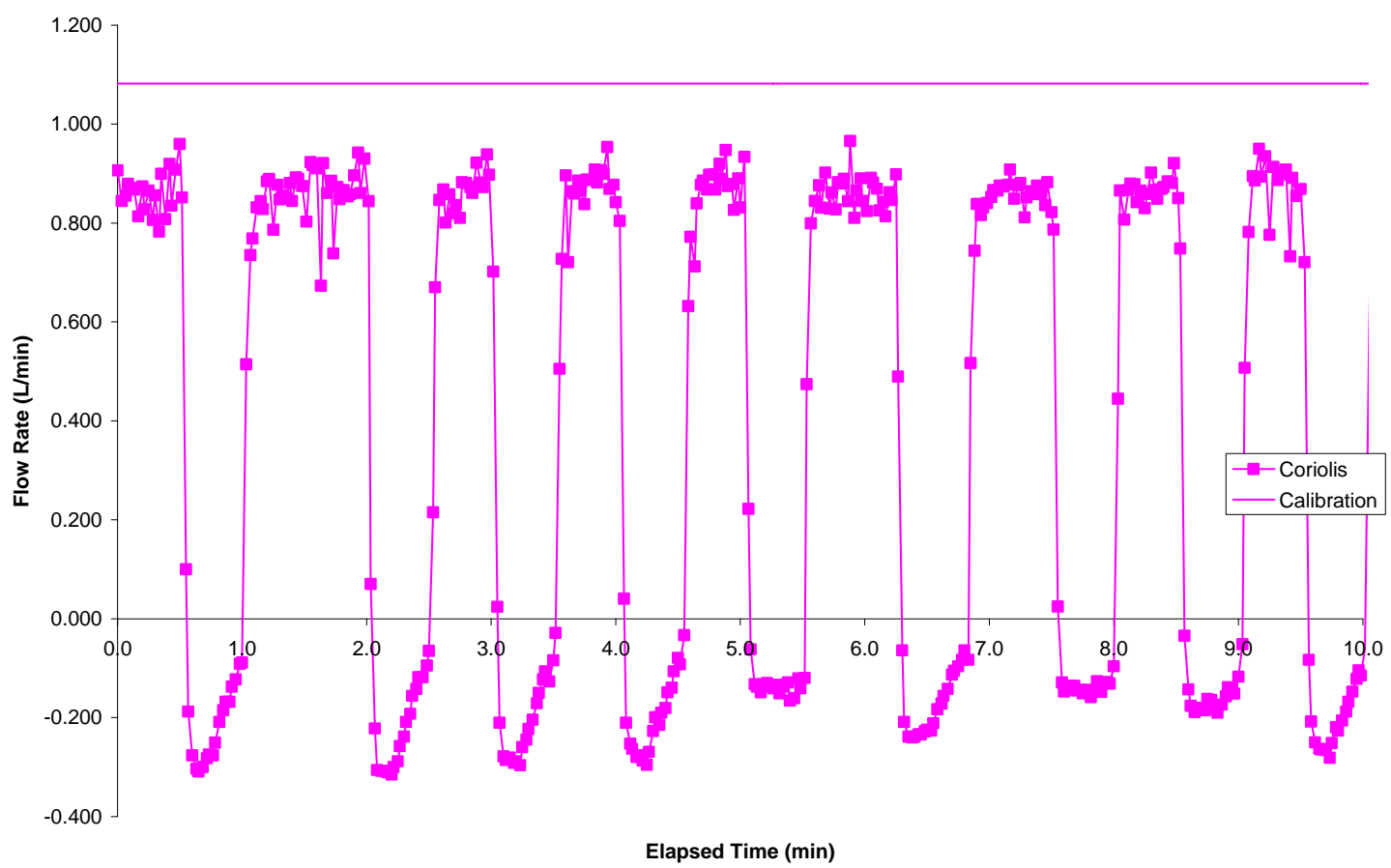

Fig. D.6. Coriolis response to pulsating flow at a pump speed of $6.0 \mathrm{~Hz}$. 


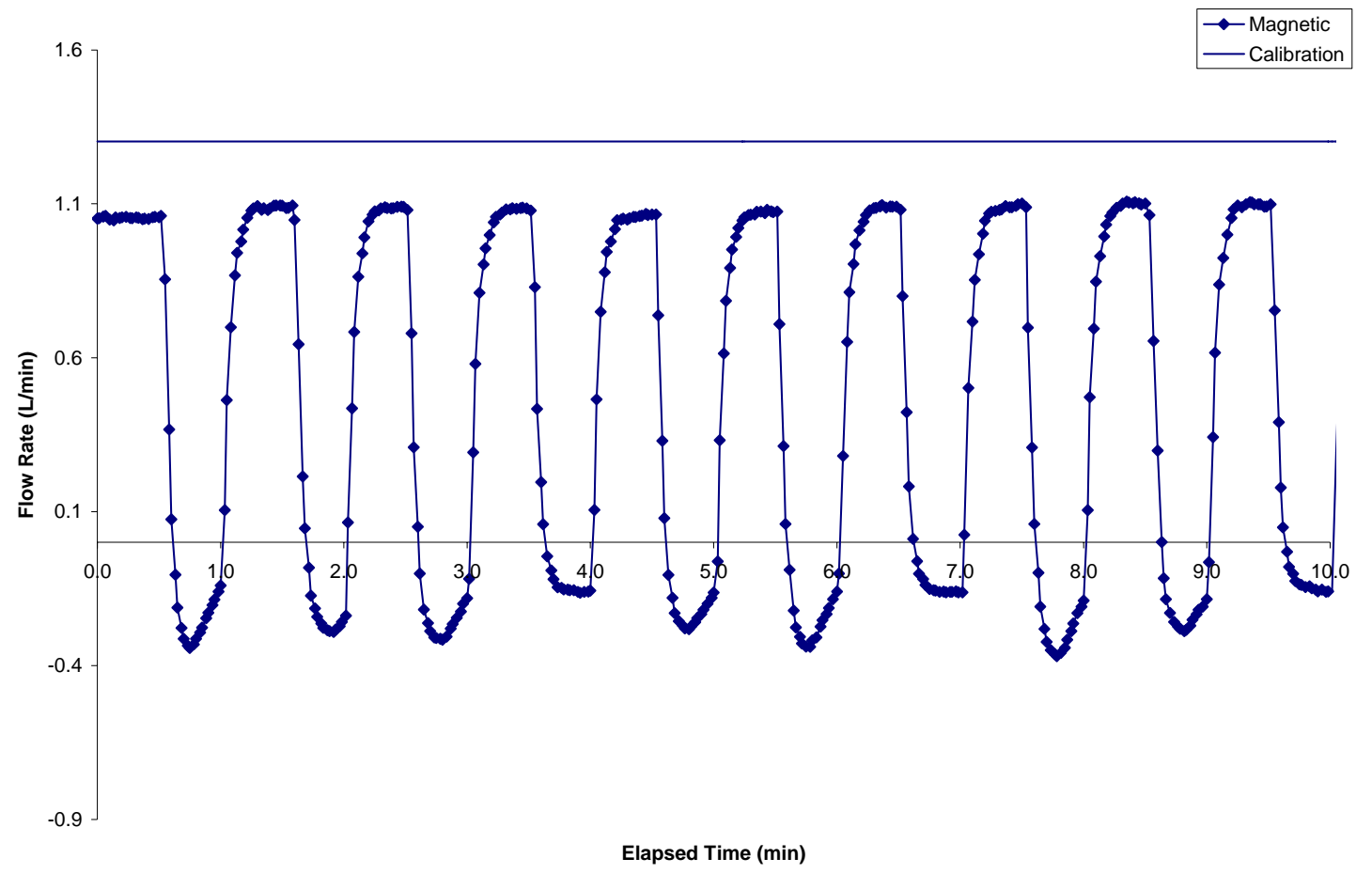

Fig. D.7. Magnetic response to pulsating flow at a pump speed of $7.2 \mathrm{~Hz}$.

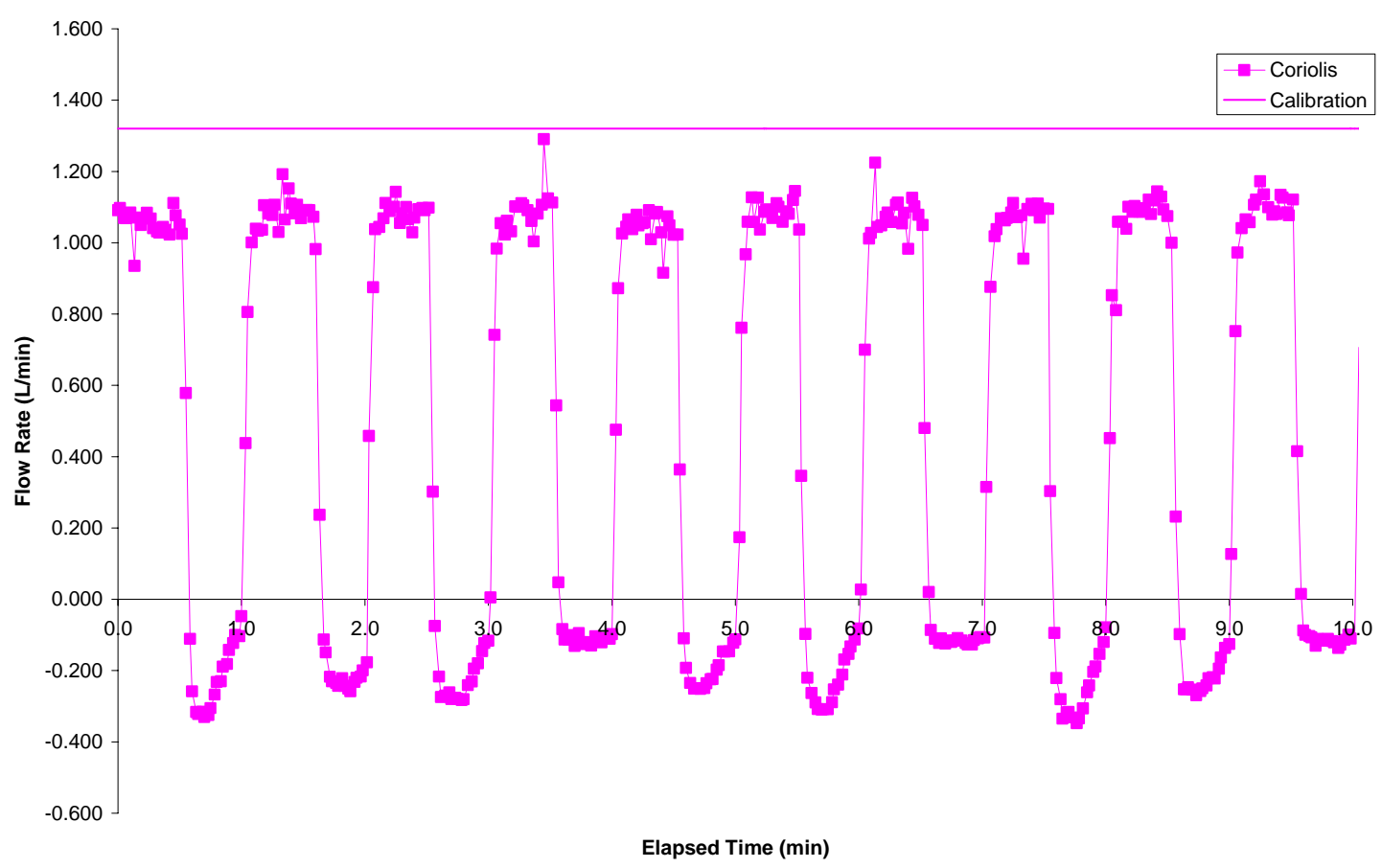

Fig. D.8. Coriolis response to pulsating flow at a pump speed of $7.2 \mathrm{~Hz}$. 


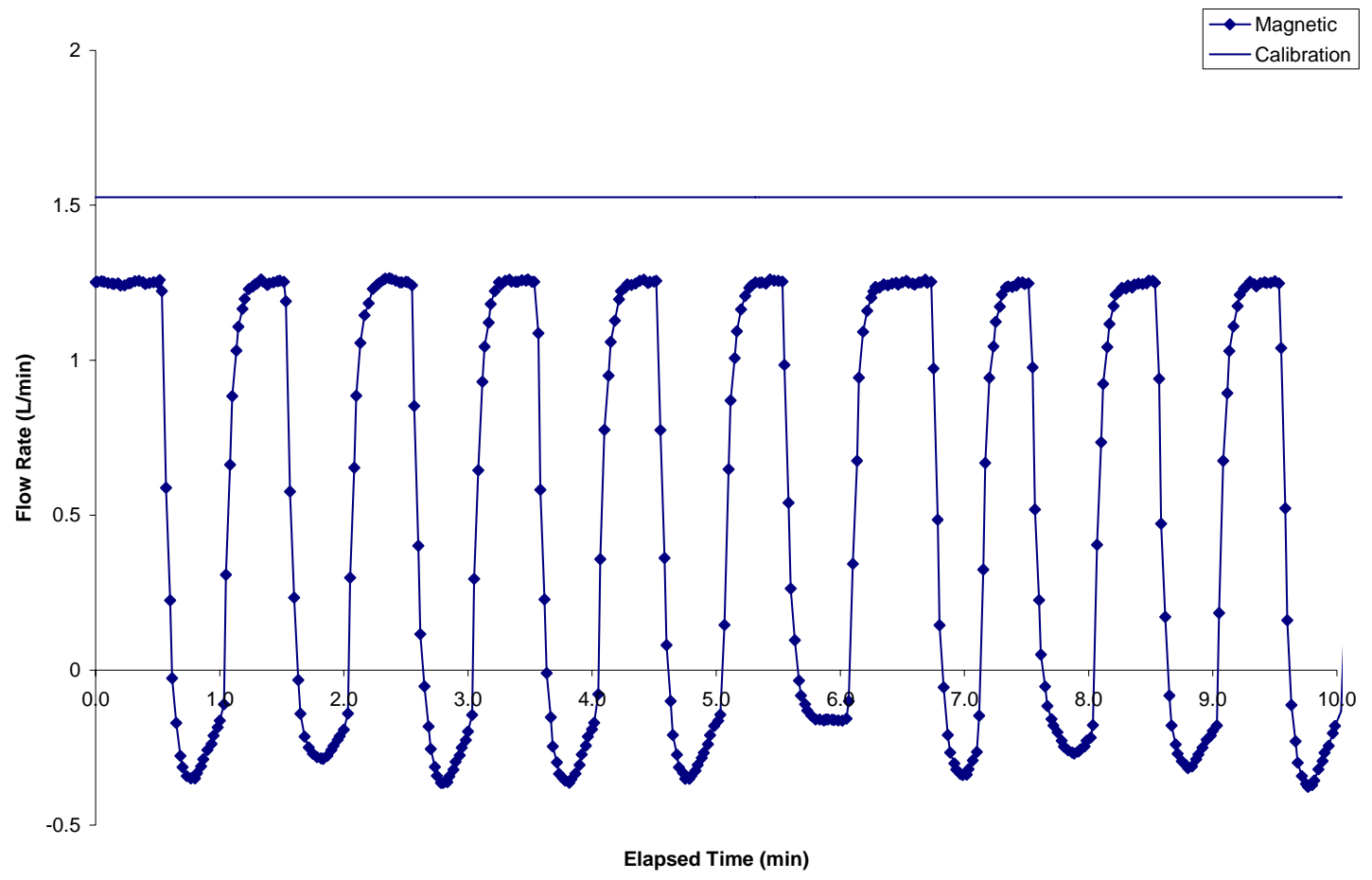

Fig. D.9. Magnetic response to pulsating flow at a pump speed of $8.3 \mathrm{~Hz}$.

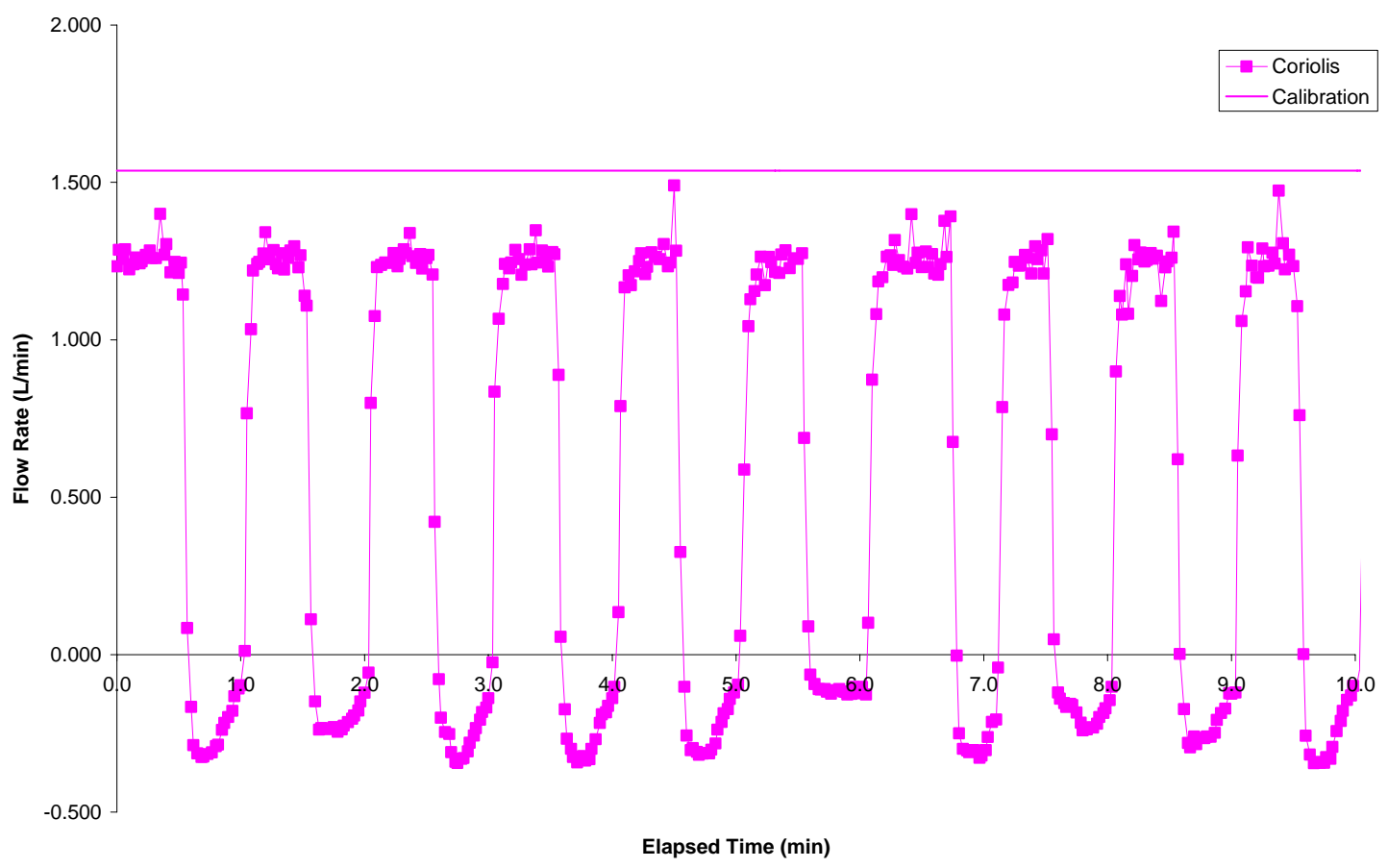

Fig. D.10. Coriolis response to pulsating flow at a pump speed of $8.3 \mathrm{~Hz}$. 


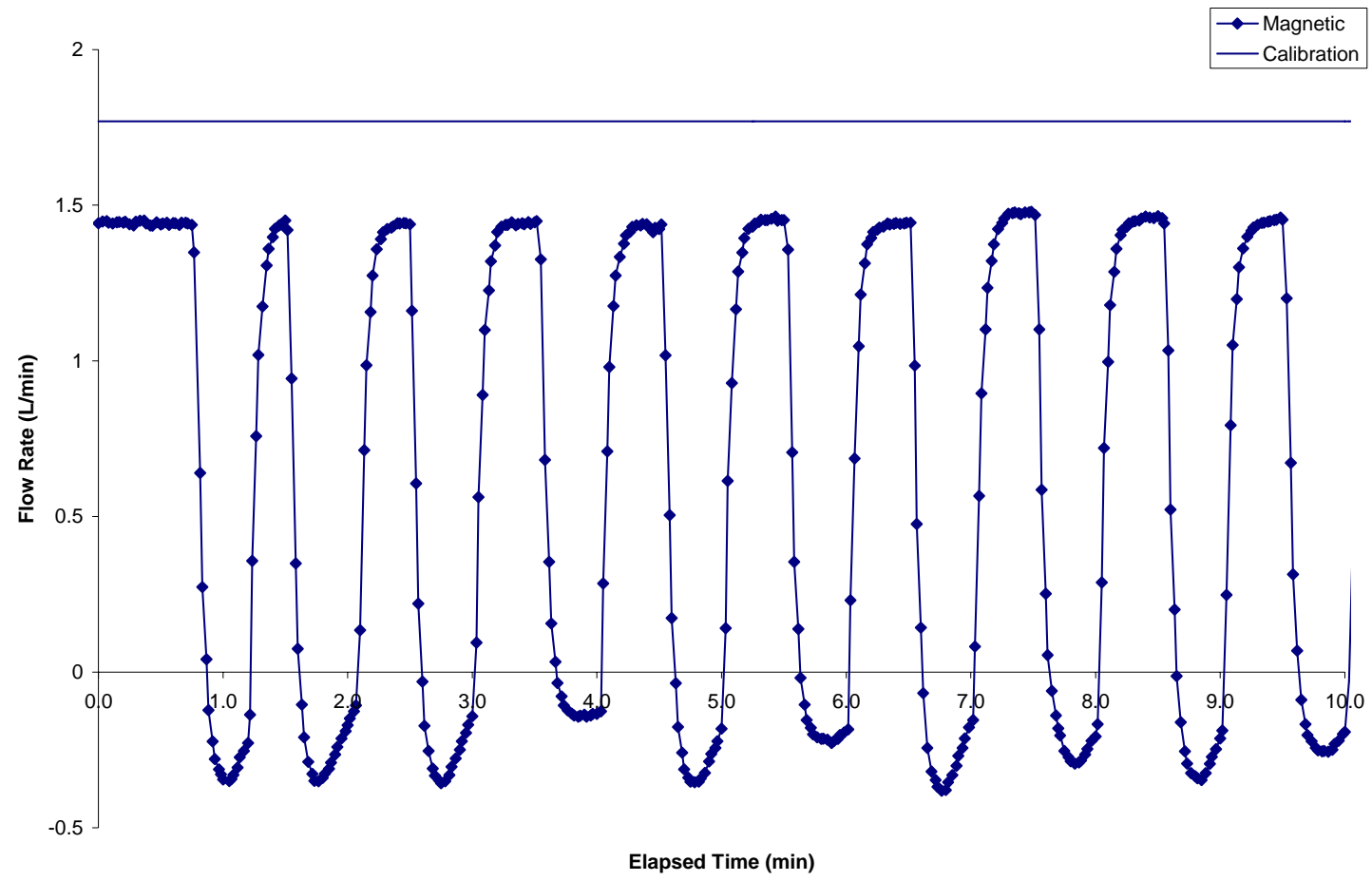

Fig. D.11. Magnetic response to pulsating flow at a pump speed of $9.5 \mathrm{~Hz}$.

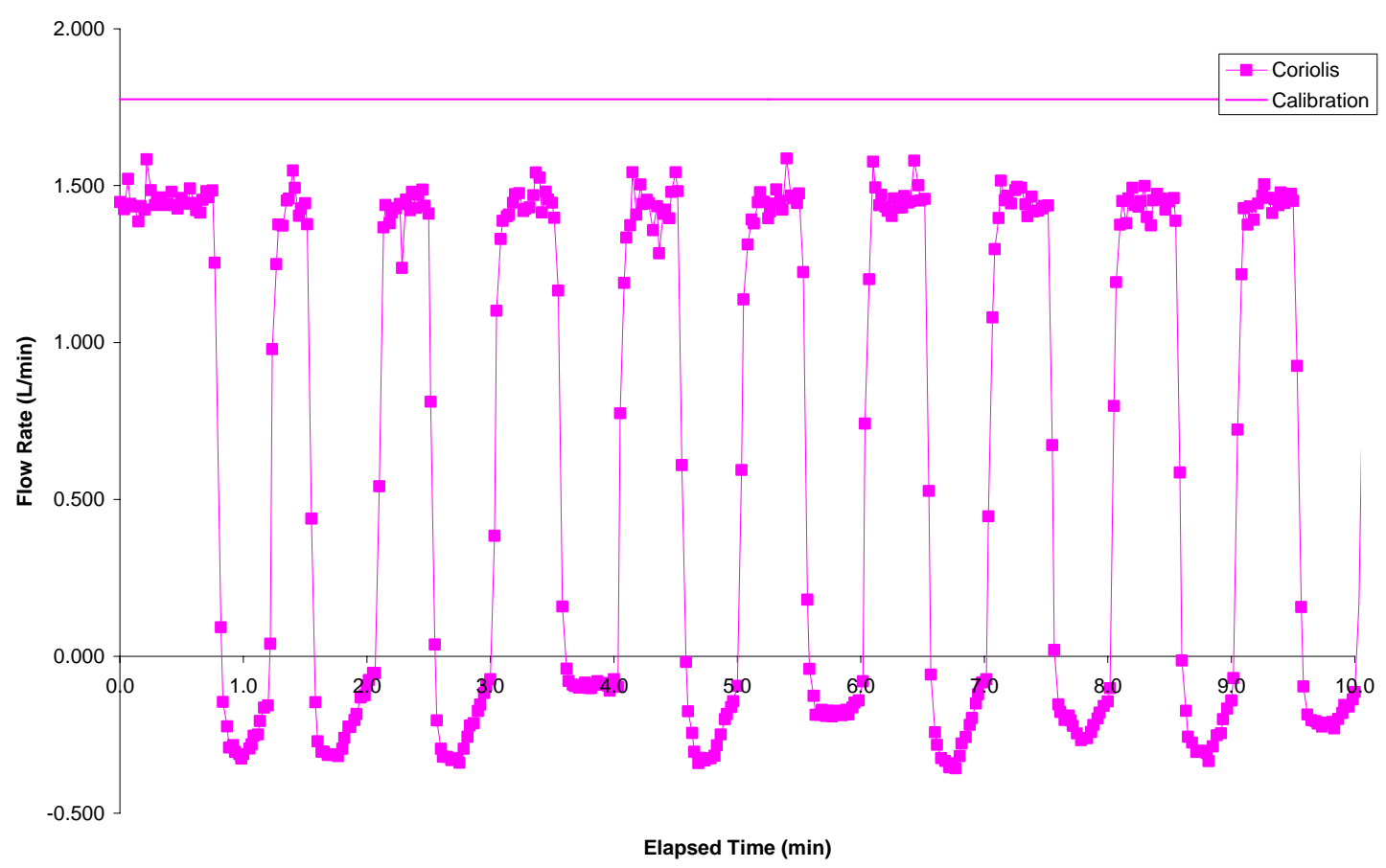

Fig. D.12. Coriolis response to pulsating flow at a pump speed of $9.5 \mathrm{~Hz}$. 


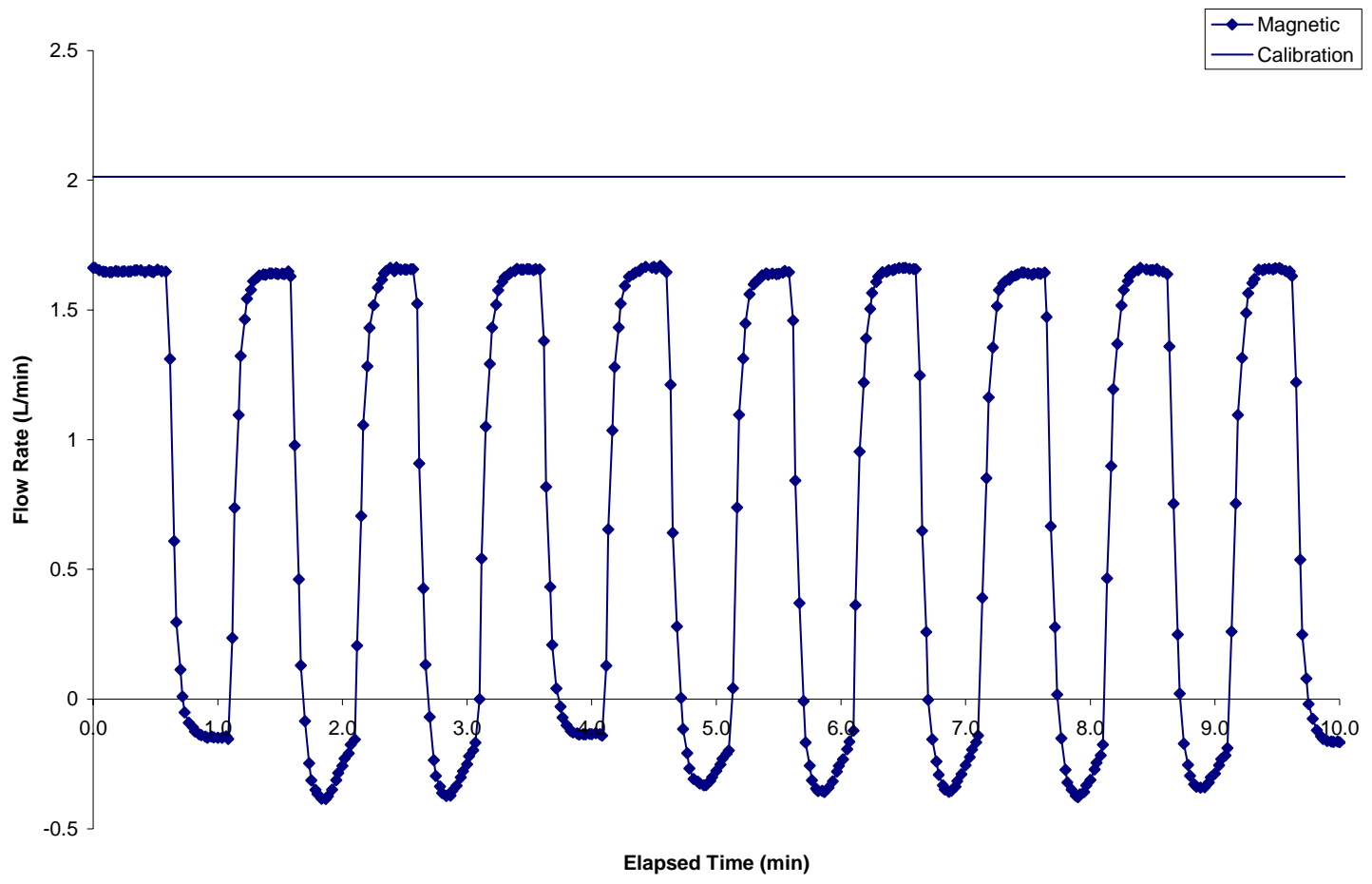

Fig. D.13. Magnetic response to pulsating flow at a pump speed of $10.7 \mathrm{~Hz}$.

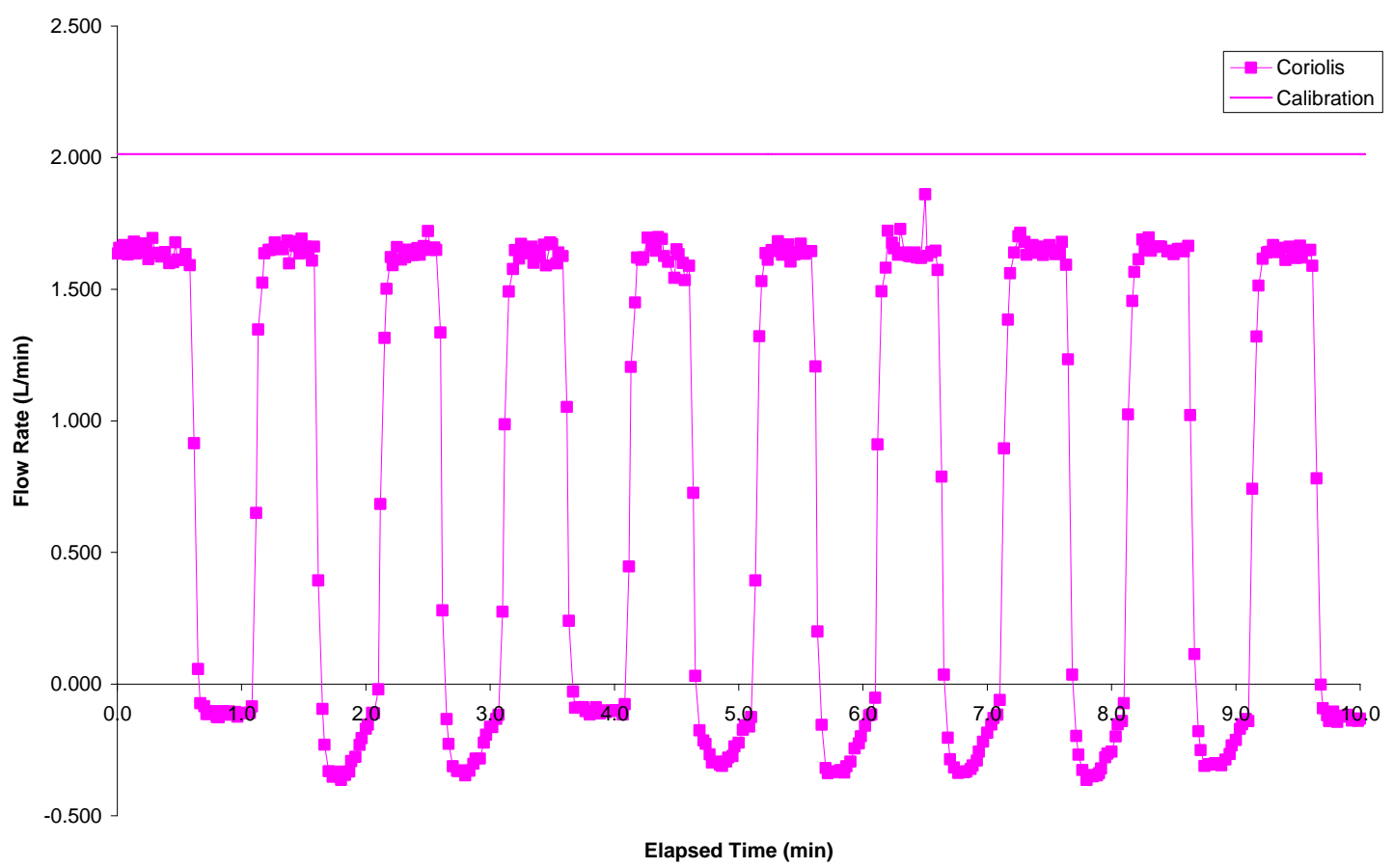

Fig. D.14. Coriolis response to pulsating flow at a pump speed of $10.7 \mathrm{~Hz}$.

D-9 


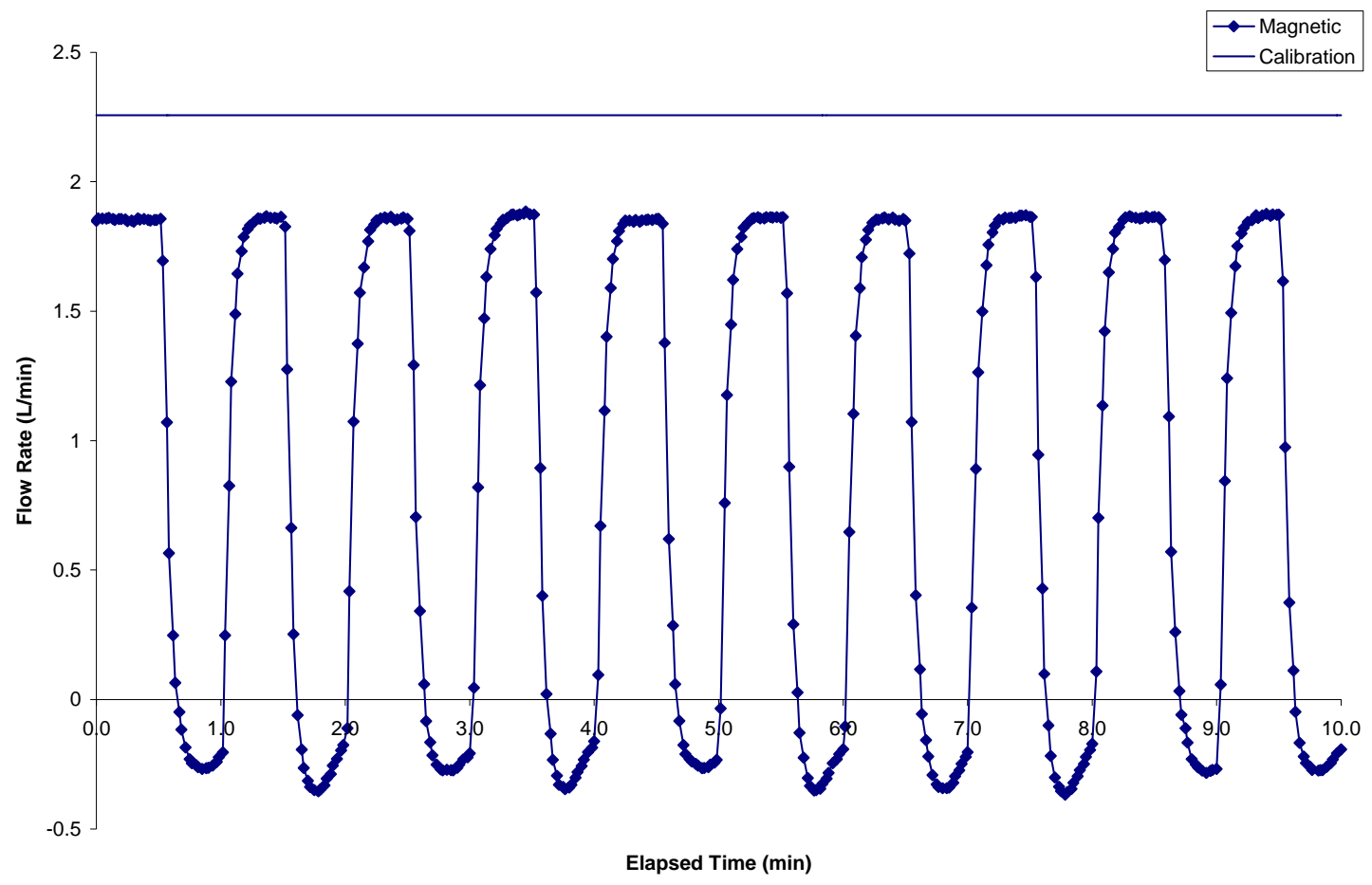

Fig. D.15. Magnetic response to pulsating flow at a pump speed of $11.9 \mathrm{~Hz}$.

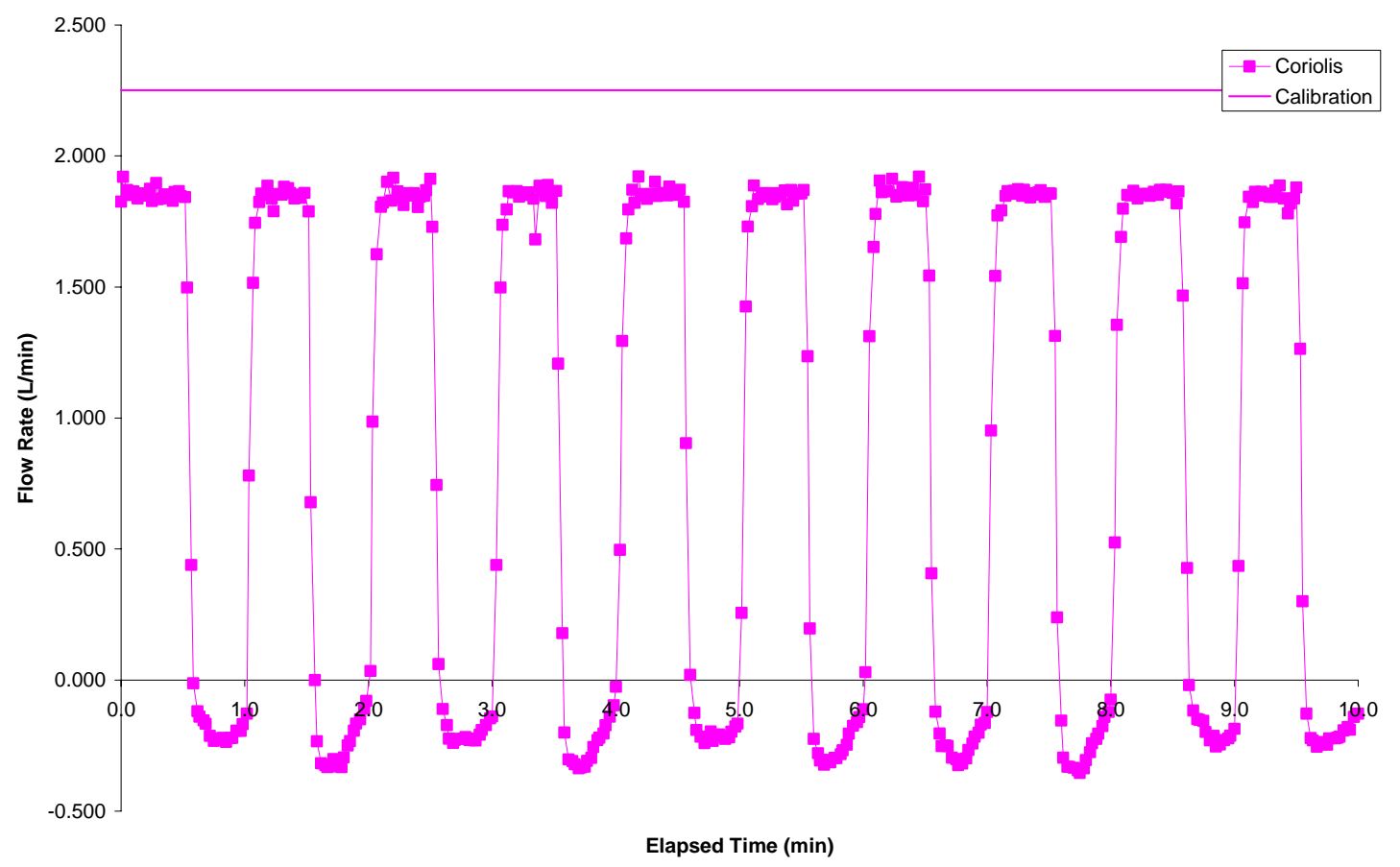

Fig. D.16. Coriolis response to pulsating flow at a pump speed of $11.9 \mathrm{~Hz}$. 
APPENDIX E

GRAPHS OF PULSATING FLOW WITH ENTRAINED AIR 



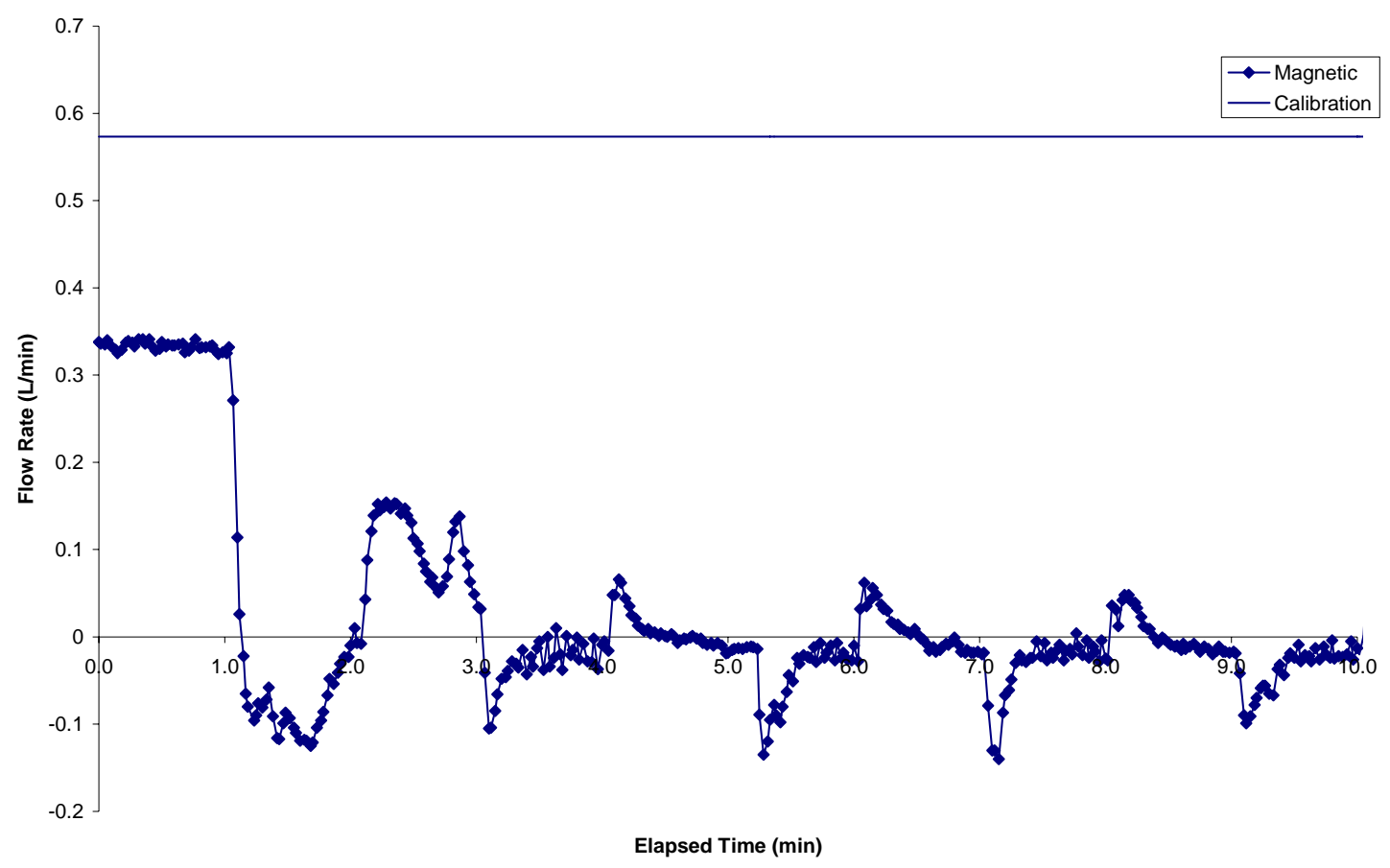

Fig. E.1. Magnetic response to pulsating flow and entrained air at a pump speed of $3.6 \mathrm{~Hz}$.

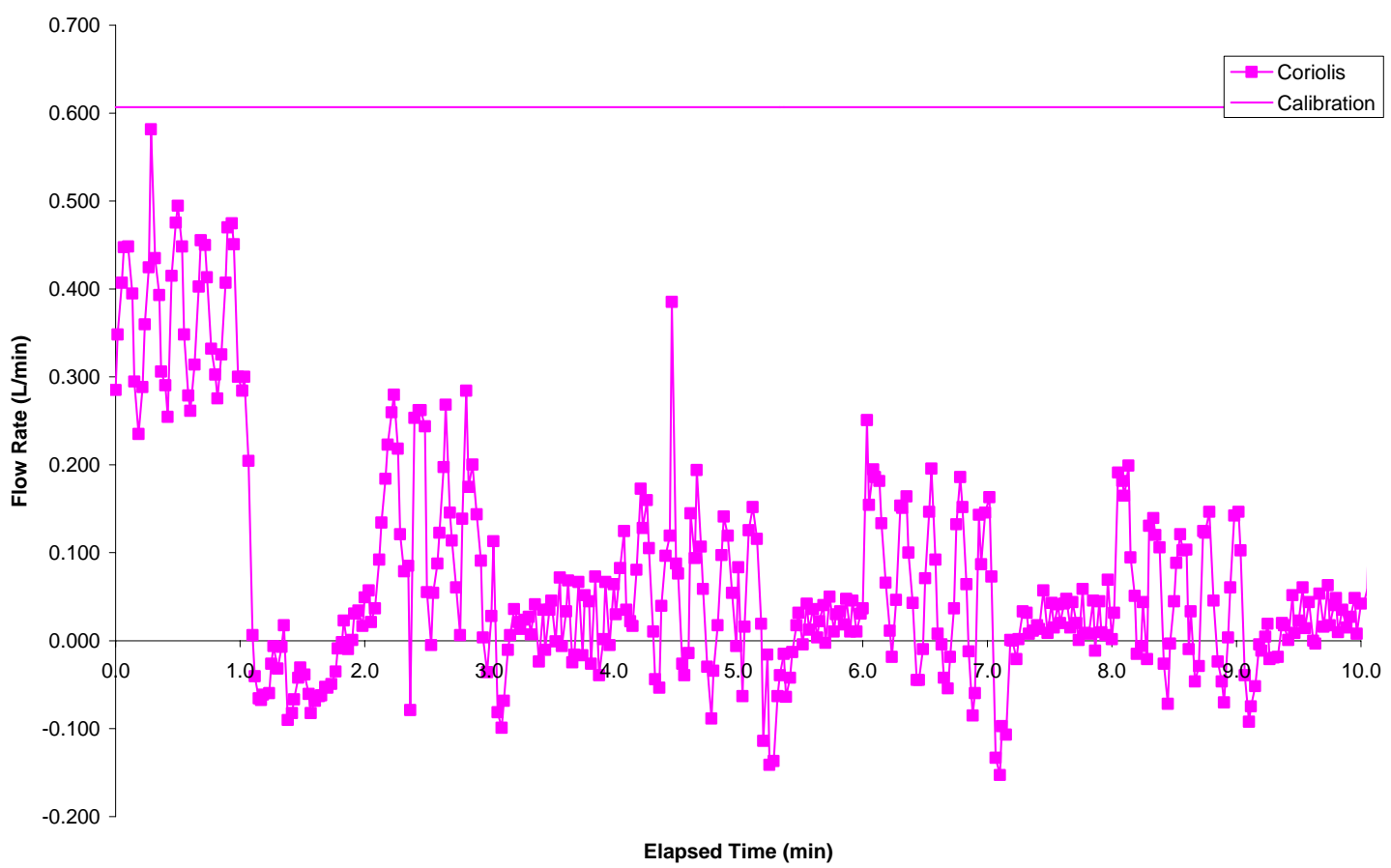

Fig. E.2. Coriolis response to pulsating flow and entrained air at a pump speed of $3.6 \mathrm{~Hz}$. 


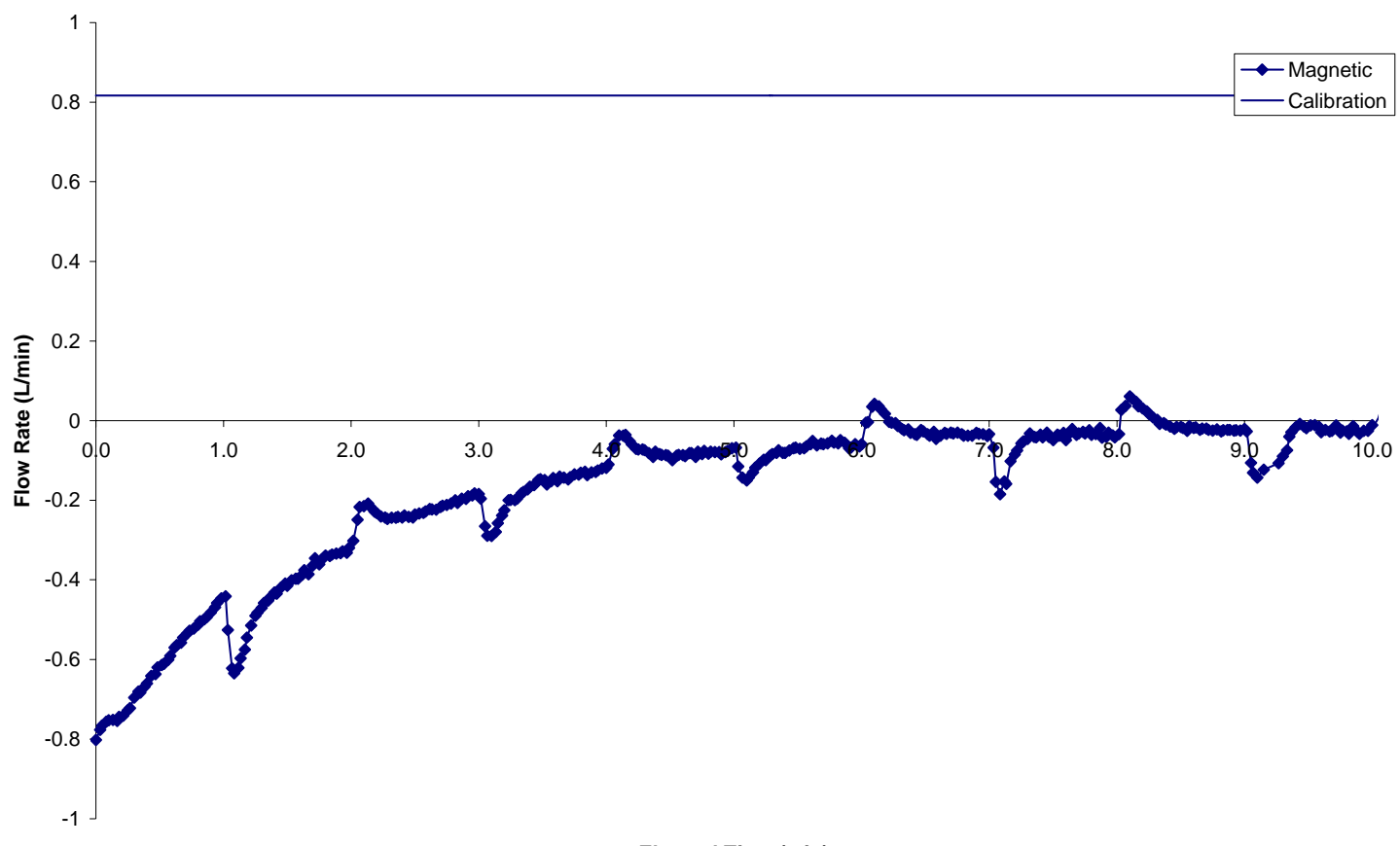

Elapsed Time (min)

Fig. E.3. Magnetic response to pulsating flow and entrained air at a pump speed of $4.8 \mathrm{~Hz}$.

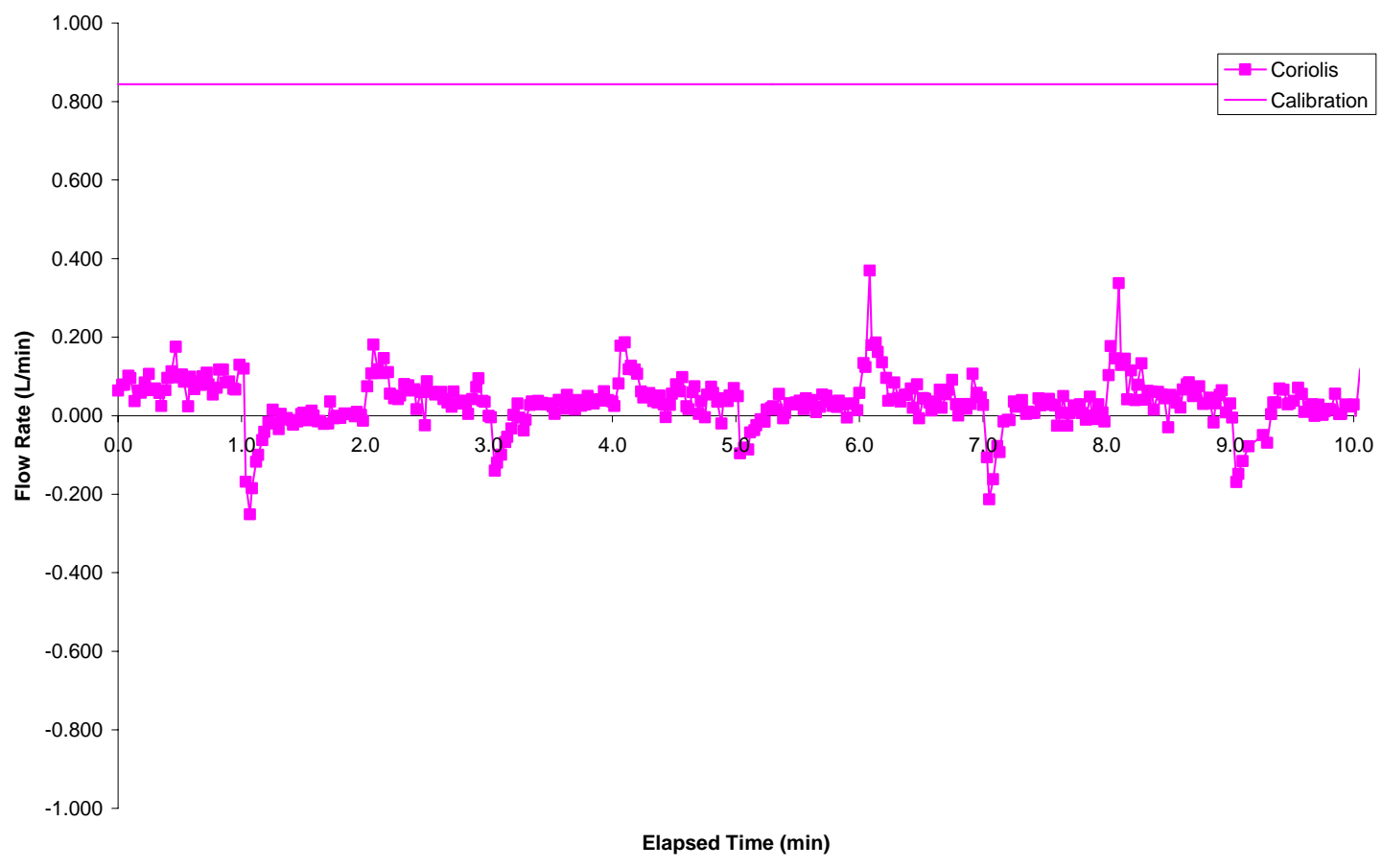

Fig. E.4. Coriolis response to pulsating flow and entrained air at a pump speed of $4.8 \mathrm{~Hz}$. 


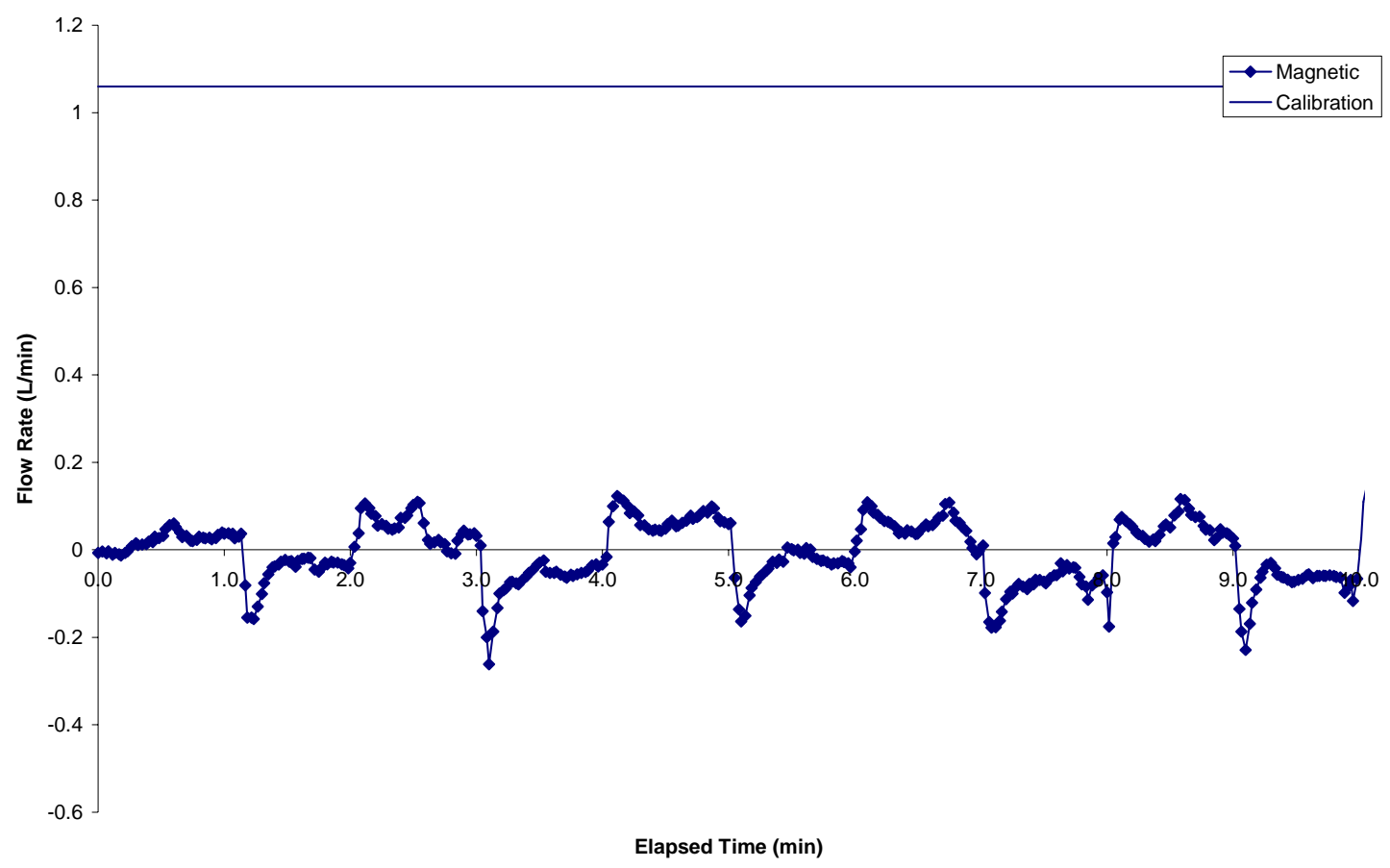

Fig. E.5. Magnetic response to pulsating flow and entrained air at a pump speed of $6.0 \mathrm{~Hz}$.

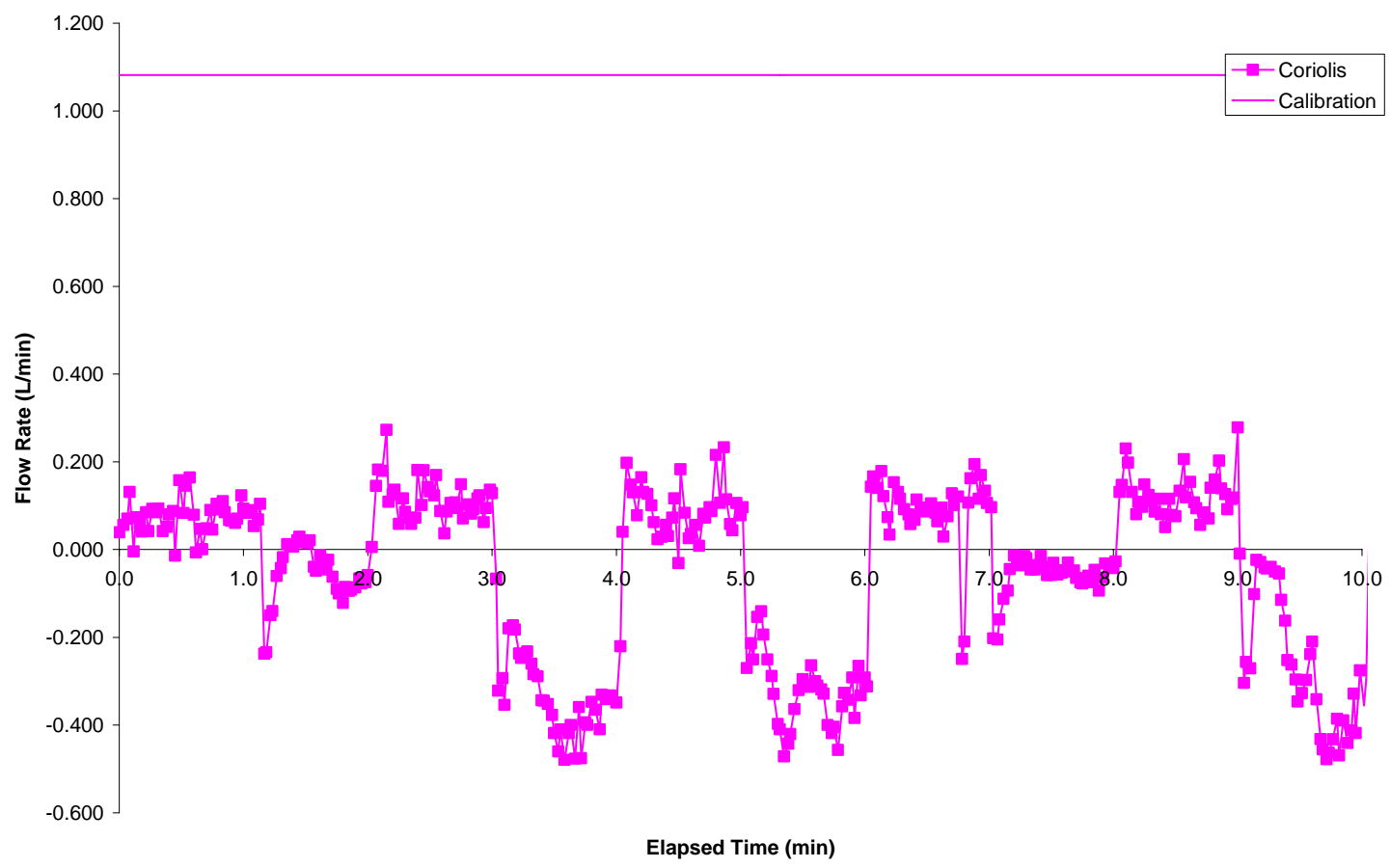

Fig. E.6. Coriolis response to pulsating flow and entrained air at a pump speed of $6.0 \mathrm{~Hz}$. 


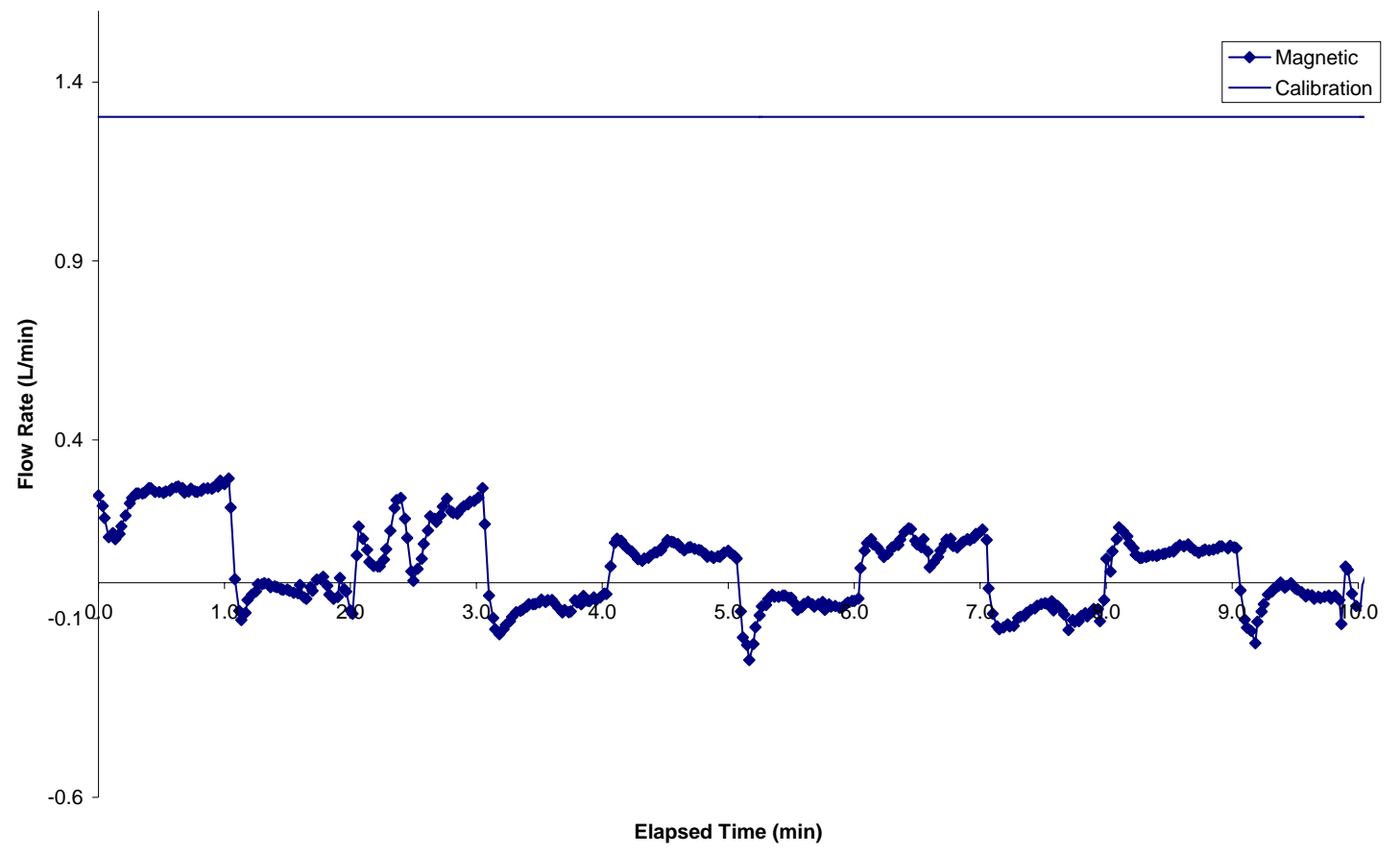

Fig. E.7. Magnetic response to pulsating flow and entrained air at a pump speed of $7.2 \mathrm{~Hz}$.

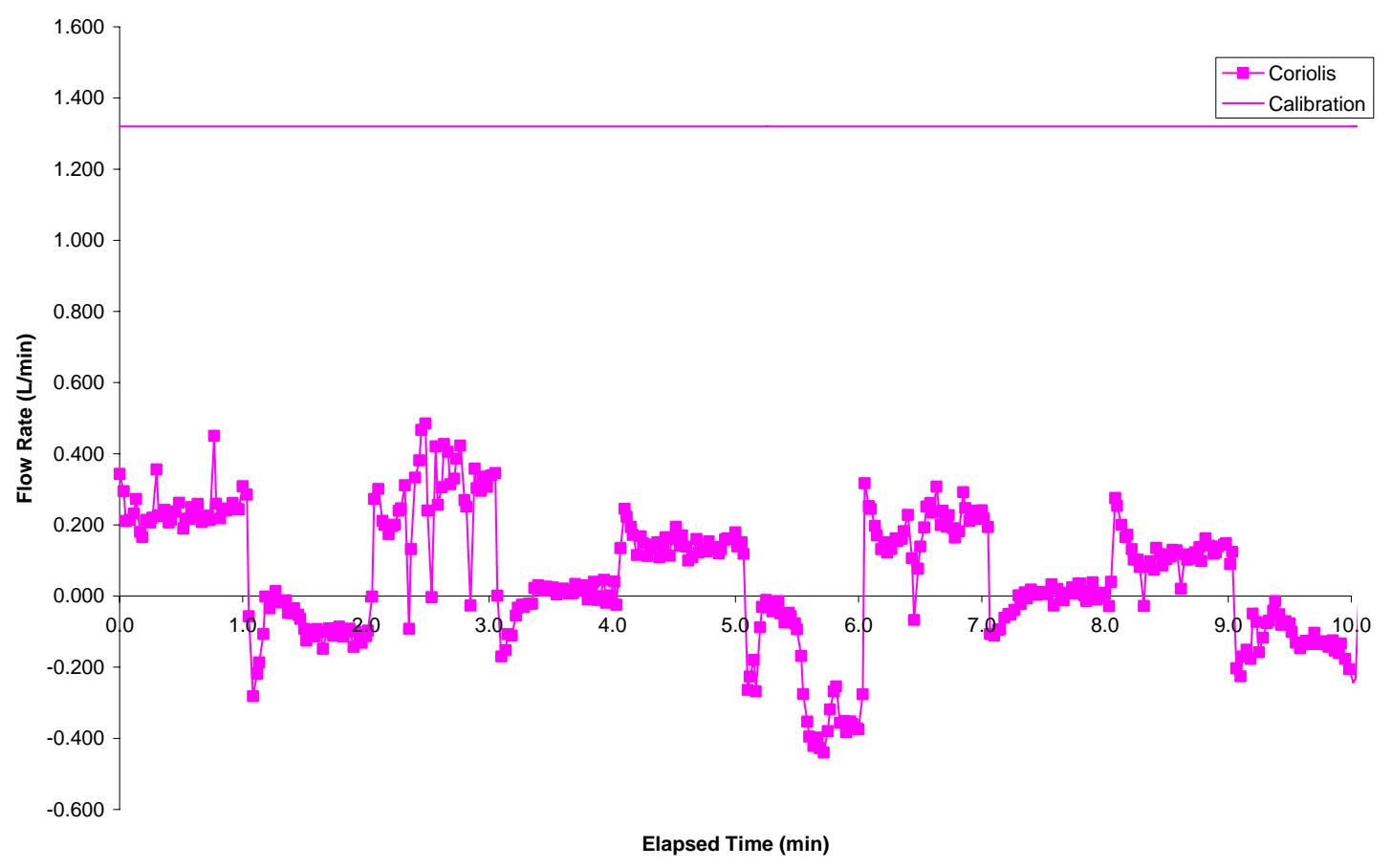

Fig. E.8. Coriolis response to pulsating flow and entrained air at a pump speed of $7.2 \mathrm{~Hz}$. 


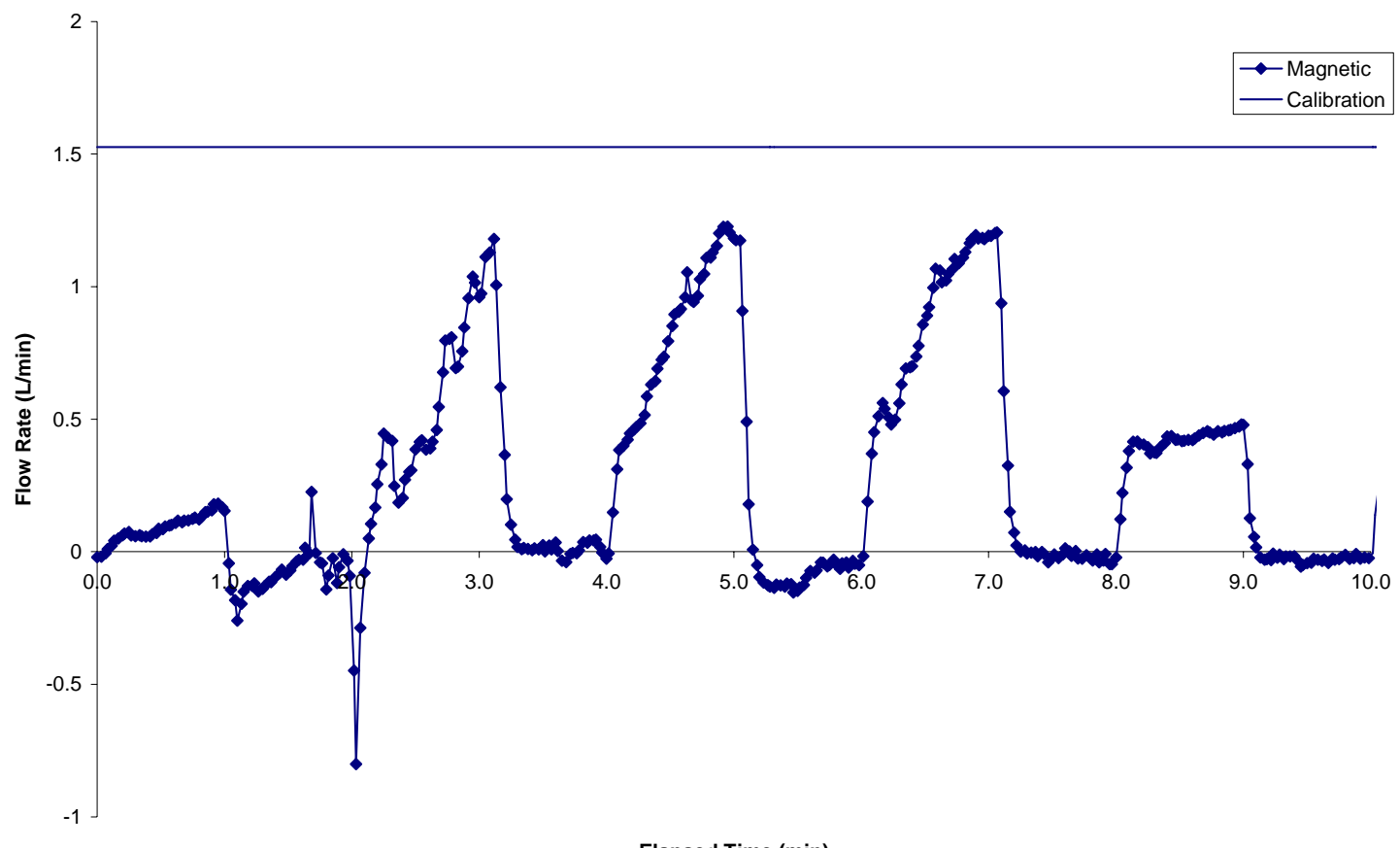

Elapsed Time ( $\mathrm{min})$

Fig. E.9. Magnetic response to pulsating flow and entrained air at a pump speed of $8.3 \mathrm{~Hz}$.

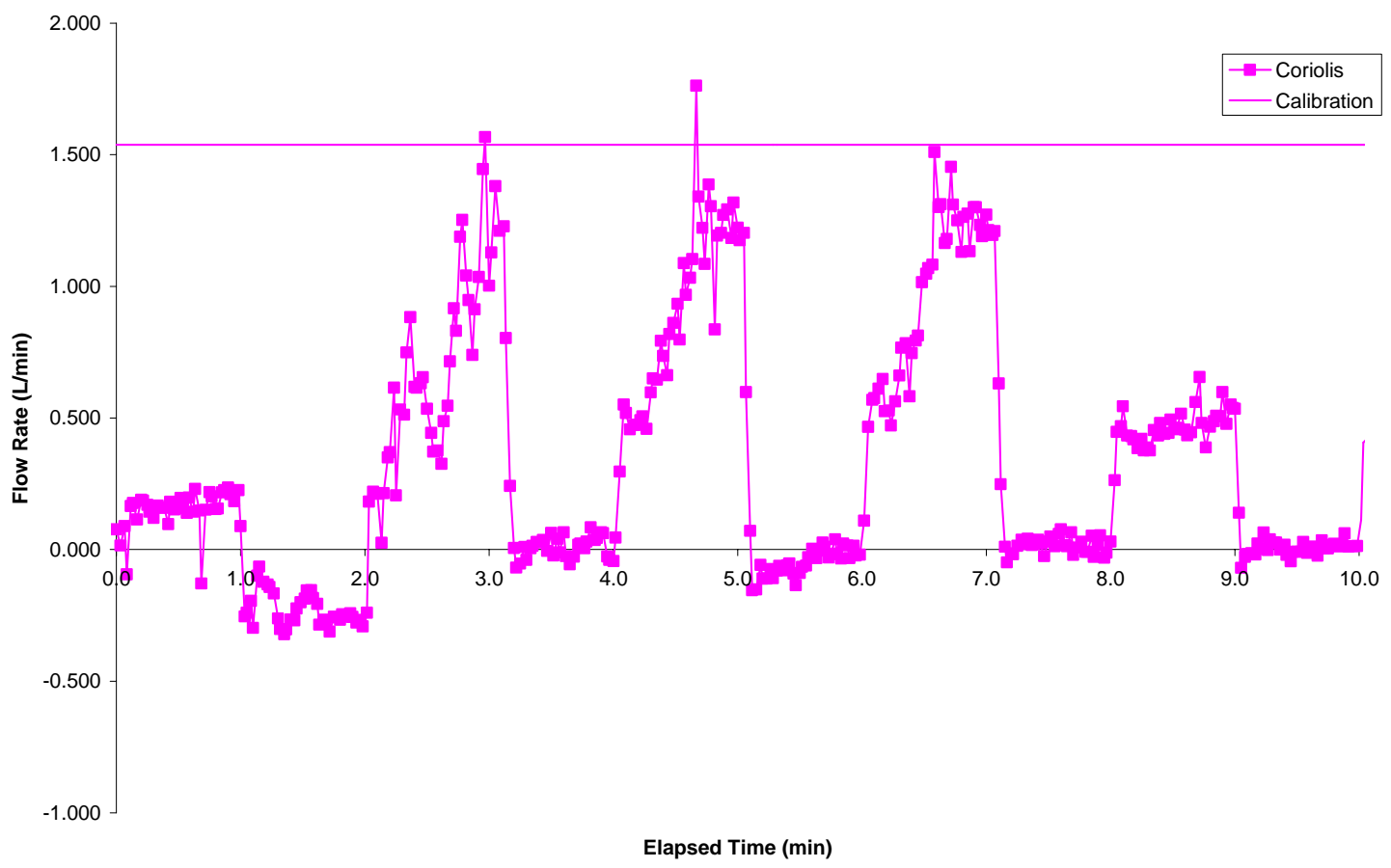

Fig. E.10. Coriolis response to pulsating flow and entrained air at a pump speed of $8.3 \mathrm{~Hz}$. 


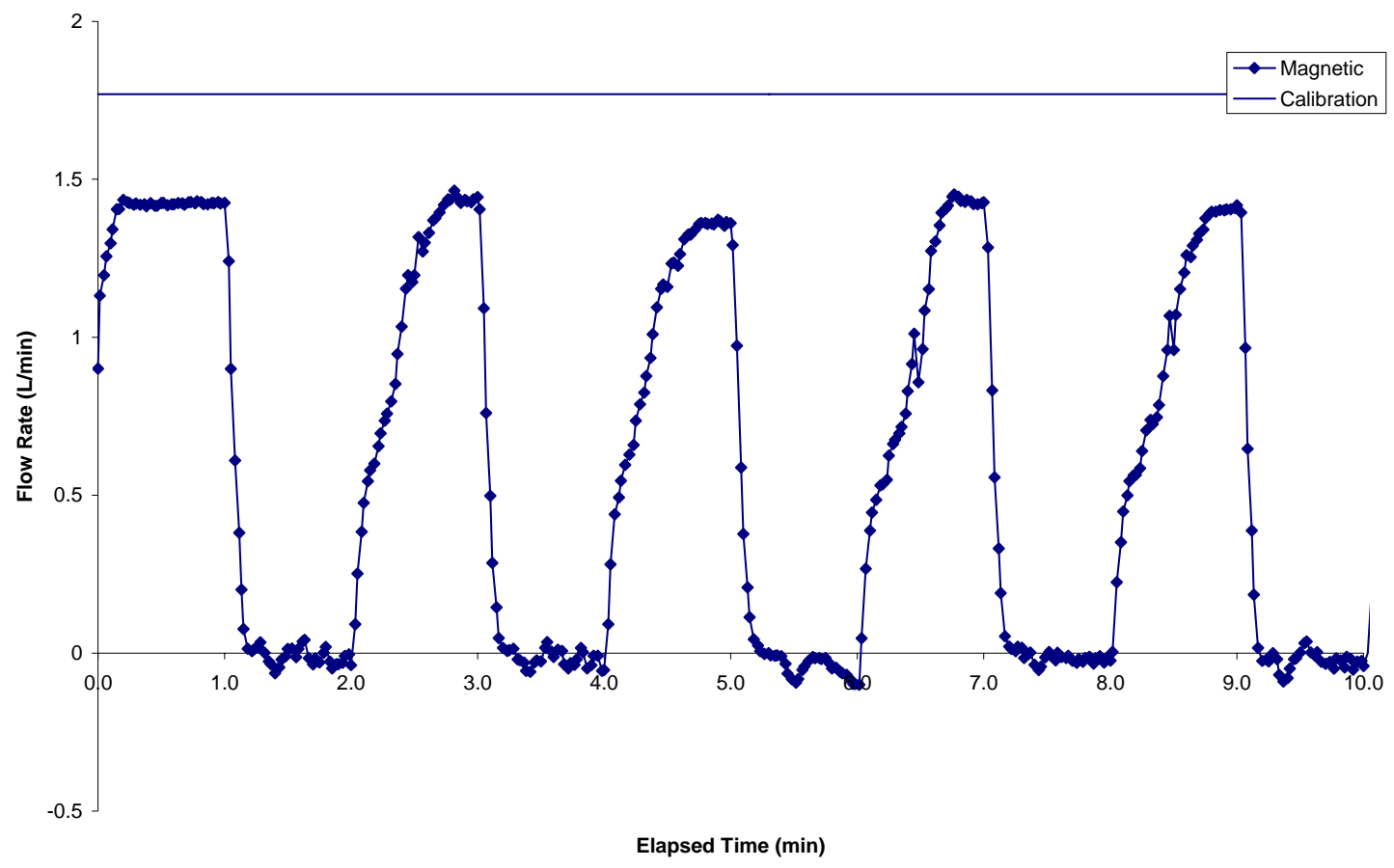

Fig. E.11. Magnetic response to pulsating flow and entrained air at a pump speed of $9.5 \mathrm{~Hz}$.

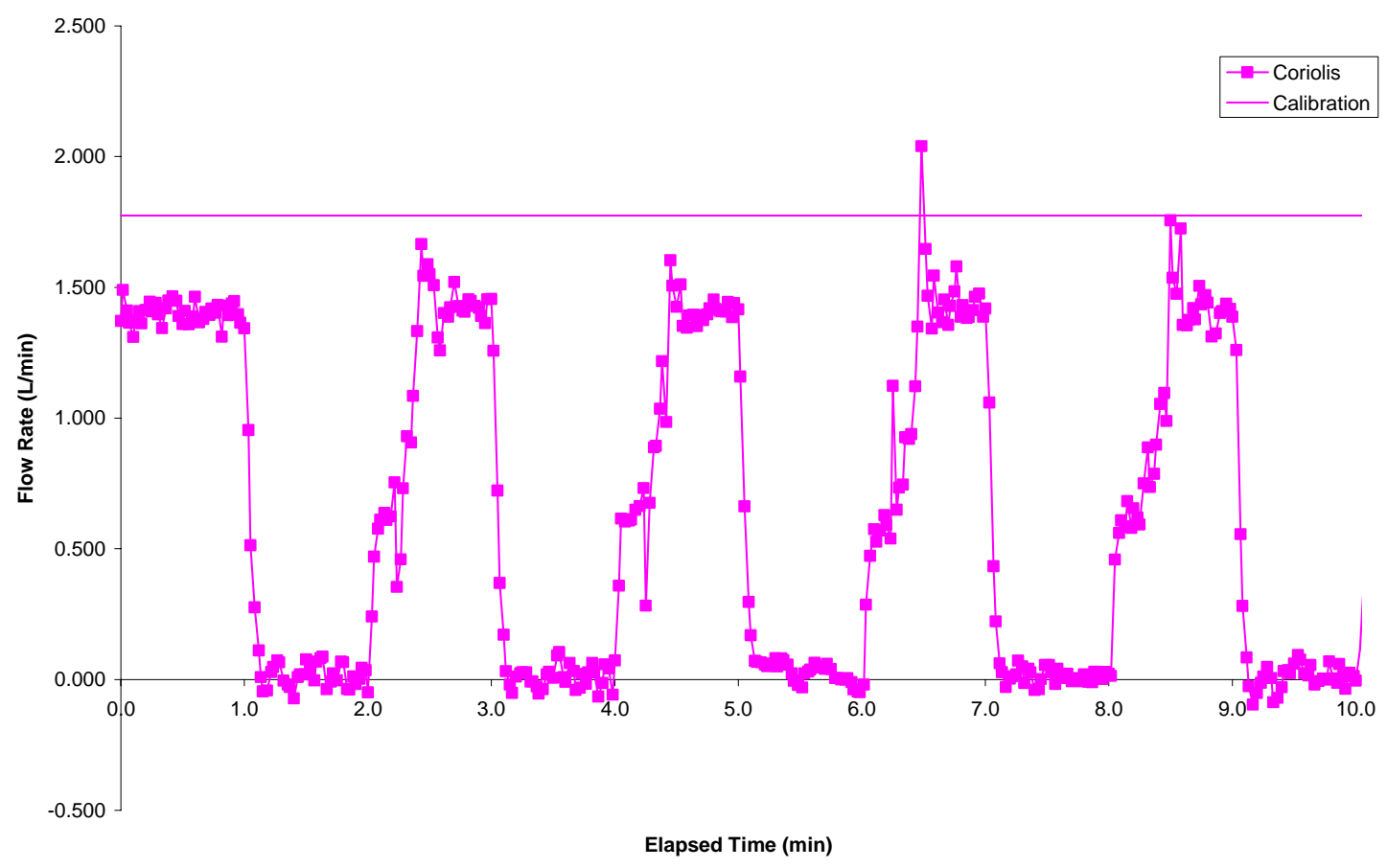

Fig. E.12. Coriolis response to pulsating flow and entrained air at a pump speed of $9.5 \mathrm{~Hz}$. 


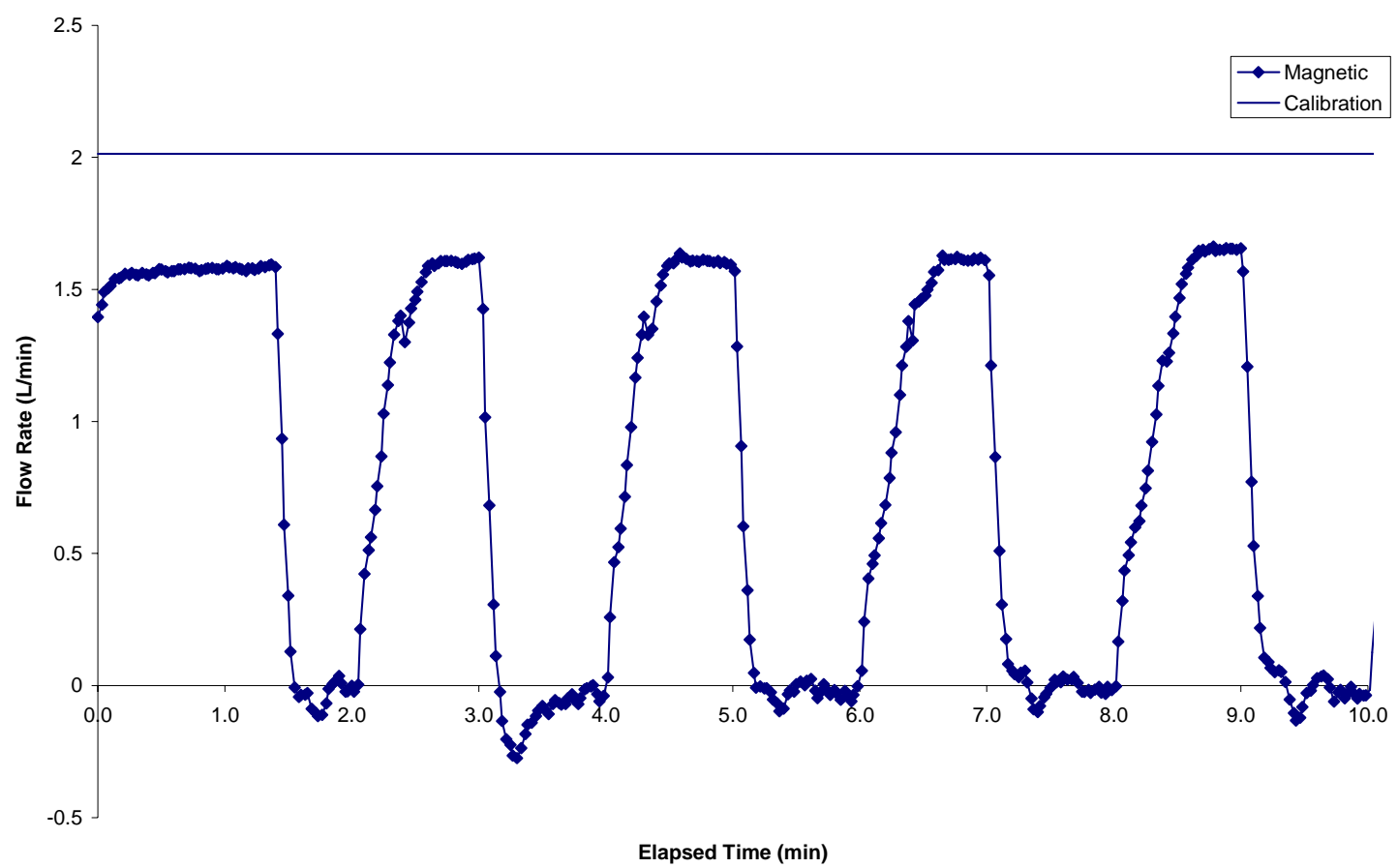

Fig. E.13. Magnetic response to pulsating flow and entrained air at a pump speed of $10.7 \mathrm{~Hz}$.

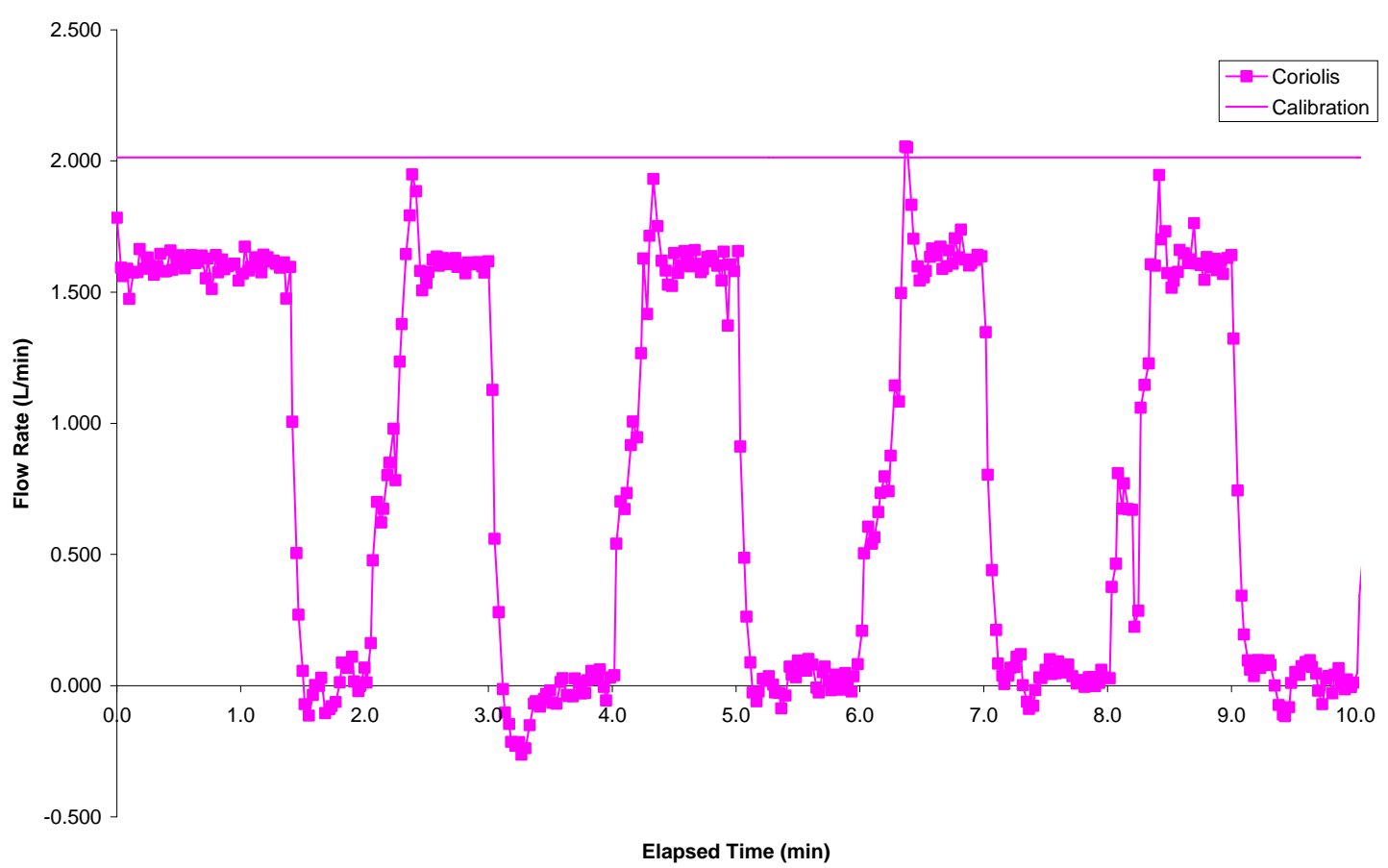

Fig. E.14. Coriolis response to pulsating flow and entrained air at a pump speed of $10.7 \mathrm{~Hz}$. 


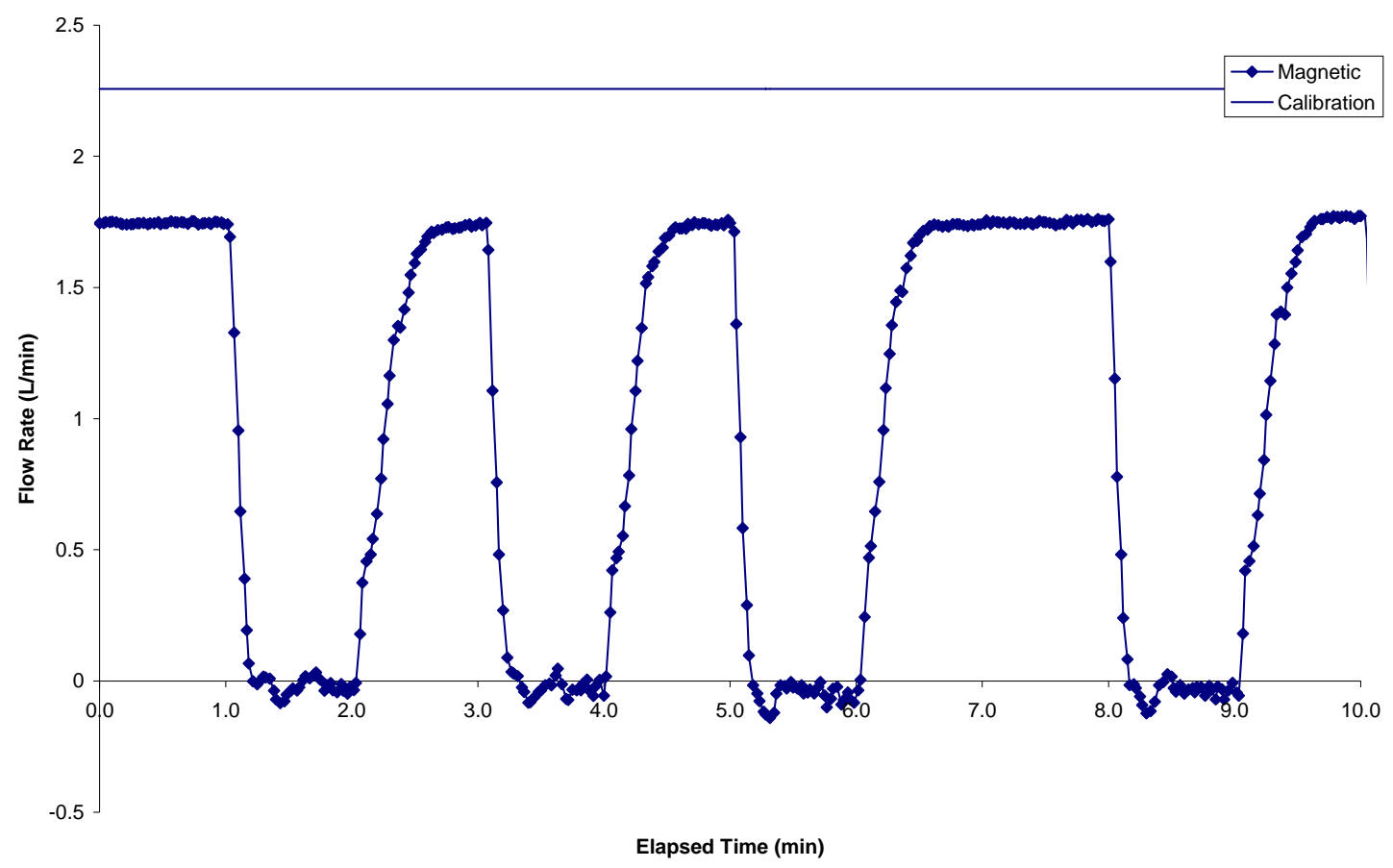

Fig. E.15. Magnetic response to pulsating flow and entrained air at a pump speed of $11.9 \mathrm{~Hz}$.

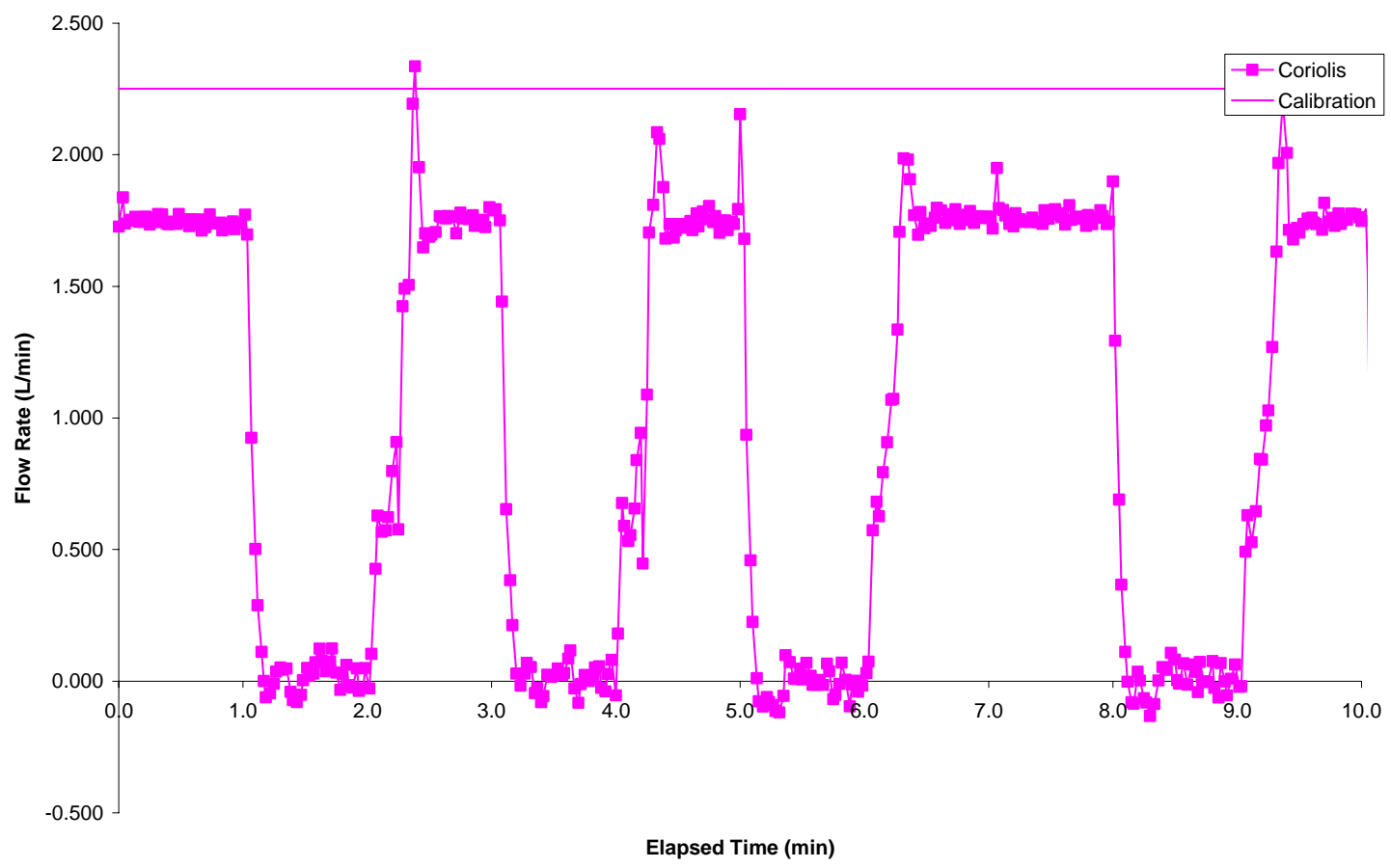

Fig. E.16. Coriolis response to pulsating flow and entrained air at a pump speed of $11.9 \mathrm{~Hz}$. 